\title{
Ice island variability in polarimetric Synthetic Aperture Radar: An exploratory analysis to improve detection and ice classification
}

\section{BY}

Gregory Lewis-Paley

A thesis submitted in partial fulfillment of the requirements for the degree of

\author{
Master of Science \\ in \\ Geography \\ Carleton University \\ Ottawa, Ontario \\ (C) 2015 \\ Gregory Lewis-Paley
}




\section{$\underline{\text { Abstract }}$}

Synthetic Aperture Radar (SAR) satellite imagery is often used to discriminate ice types and identify glacial ice hazards to marine operations. This 'data-mining' study examined 123 polarimetric SAR variables in 70 RADARSAT-2 images to assess their utility in separating ice islands (large tabular icebergs) from other ocean covers. Difference of means tests identified five SAR variables to separate each ocean cover. Further SAR variables were found using forward-selection in Redundancy Analysis (RDA). RDA also identified incidence angle, air temperature and wind speed as primary confounding factors for all assessed ocean covers. Support Vector Machine classification using five SAR variables was used to develop an ocean cover classification model. This technique competently distinguished open water, first- and multi-year ice, but could not discriminate ice islands. The selected SAR variables, many not previously investigated, warrant further study and the RDA approach showed promise to guide future development of remote sensing classifiers. 


\section{Acknowledgements}

This work would not have been possible without funding and support from the Canadian Foundation for Innovation, the Ontario Research Fund, ArcticNet, Natural Sciences and Engineering Research Council of Canada (NSERC) and the Government Related Initiatives Program (GRIP) for the "RADARSAT-2 mode selection for maritime surveillance" report which formed the initial inspiration for this research. The Canadian Ice Service (CIS) provided RADARSAT-2 scenes under Carleton-CIS Joint Project Agreements. RADARSAT-2 data and products used in this study are copyrighted by MacDonald Dettwiler and Associates Ltd (2009, 2010, 2011, 2012, 2013, 2014) with all rights reserved. RADARSAT is an official mark of the Canadian Space Agency.

The CIS provided satellite beacons that tracked ice islands and assisted image acquisition. C-CORE also provided a satellite beacon for ice island tracking (2011) and Canatec and Associates provided several beacons as well (2009 and 2010). ArcticNet provided funding and access to the CCGS Amundsen to place these beacons (2011, 2012, 2013 and 2014). The Polar Continental Shelf Program also supplied in-kind logistical support (2009 and 2010).

I would like to acknowledge the Free and Open Source Software movement which allowed me to script my research using the best tools for the job, with the ability when needed to inspect the underlying code and ensure various algorithms were working as anticipated.

I would like to thank Derek Mueller, who has provided ongoing guidance of my 
research. His leadership and direction allowed me to start with a topic I initially knew little about and gave me the opportunity to expand my knowledge and apply my skills. Doug King provided further research direction and an outside critical assessment of the limitations of this research as a member of my committee. I would also like to thank Professor Brigitte Leblon at the University of New Brunswick whose course entitled "Advanced Studies in Radar Polarimetry” allowed for an in-depth understanding of the subject at hand. I also want to acknowledge support from Anna Crawford who herself visited several of the ice islands in the study to deploy tracking beacons. She also provided ongoing guidance with her invaluable practical ice island experience.

I would also like to thank my parents for imparting to me a wealth of knowledge, and getting me excited about computers and programming from a young age. Without that grounding, none of this would be possible. Finally, I'd like to thank my beautiful wife Brittany for sticking with me through thick and thin, and not rolling her eyes too much when I had an amazing new idea I had to try. 


\section{Table of Contents}

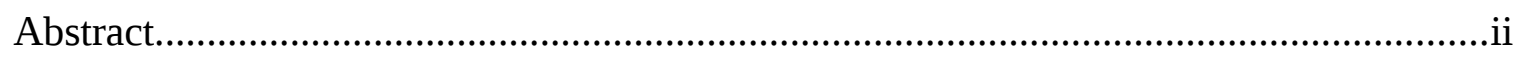

Acknowledgements.................................................................................................ii

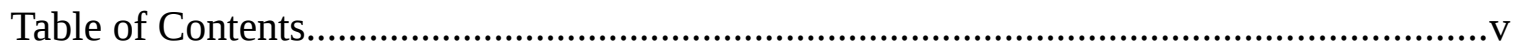

List of Figures............................................................................................................

List of Tables..........................................................................................................

List of Appendices.................................................................................................

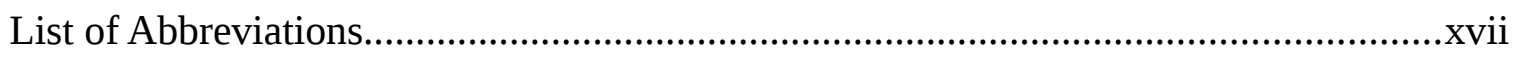

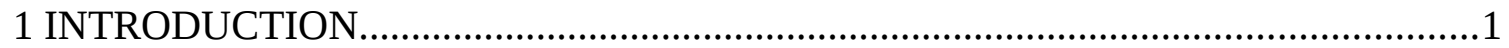

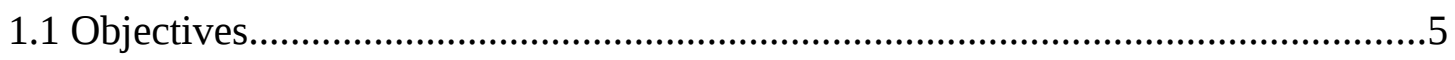

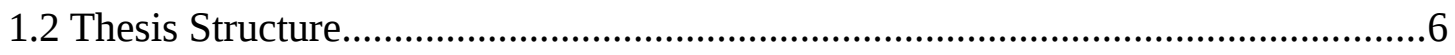

2 LITERATURE REVIEW..............................................................................

2.1 SAR

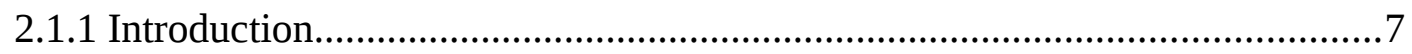

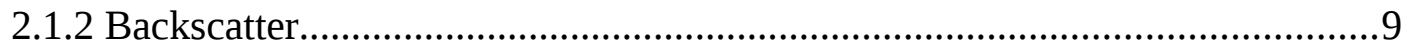

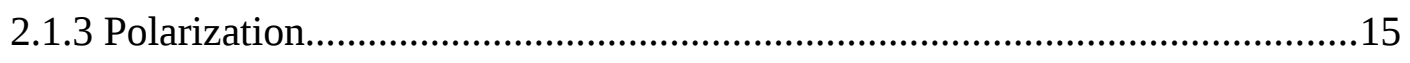

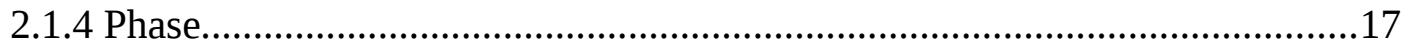

2.1.5 SAR Decompositions and Variables...............................................................18

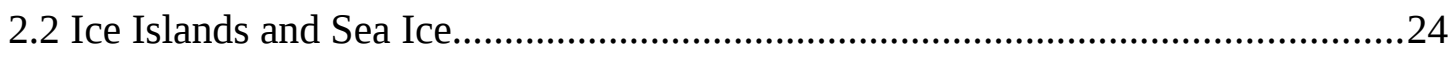

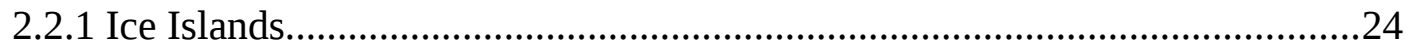

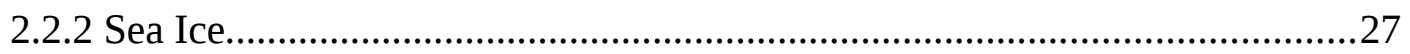

2.3 SAR Interaction with Ocean Covers and Classification.........................................30

2.3.1 Ice Islands.....................................................................................................

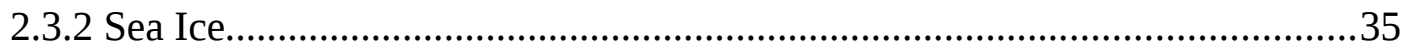

2.4 Background to the Analytical and Mapping Techniques used in this Research.....36

2.4.1 Separability Measures to Assess SAR Variables................................................37

2.4.2 Direct Gradient Analysis..........................................................................38

2.4.3 Segmentation and Classification Techniques................................................42 
3.1 Radar Data and Site Selection......................................................................49

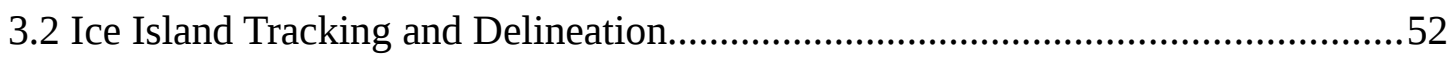

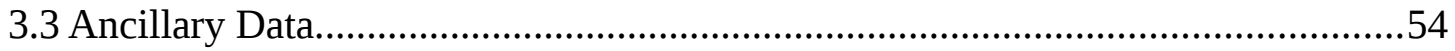

3.3.1 Identity Variable - CIS Ice Type.............................................................55

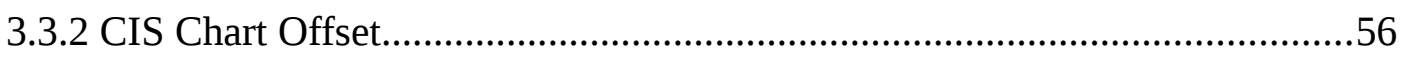

3.3.3 Environmental Variables - Wind / temperature...........................................57

3.3.4 Geometry Variable - Azimuth Angle to Surface Ridges...............................57

3.3.5 Geometry Variable - Incidence Angle..................................................59

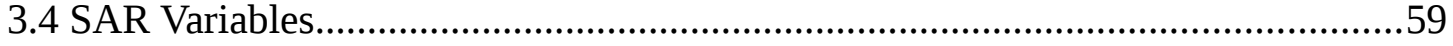

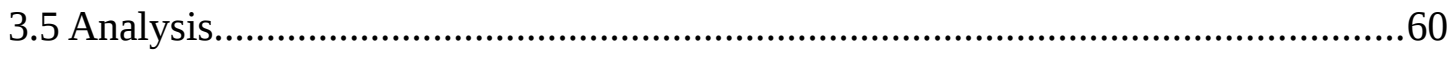

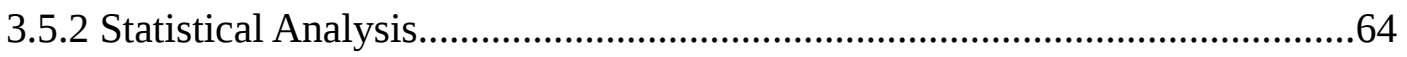

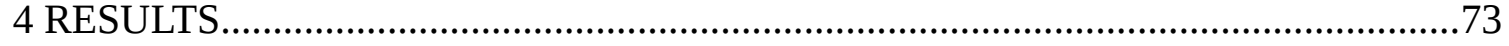

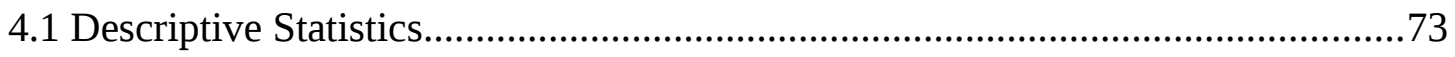

4.1.1 SAR Geometry and Environmental Data...............................................73

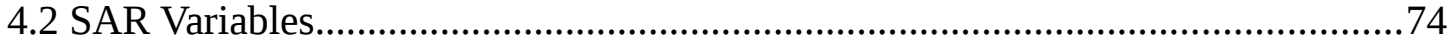

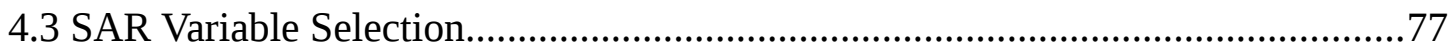

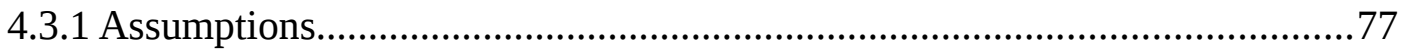

4.3.2 Overall Separability of SAR Variables....................................................77

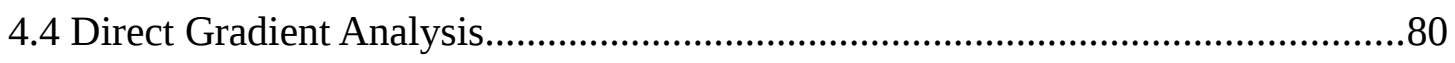

4.4.1 Selecting CCA or RDA and Significance tests.........................................8

4.4.2 Direct Gradient Analysis of RDA Selected SAR Variables...........................81

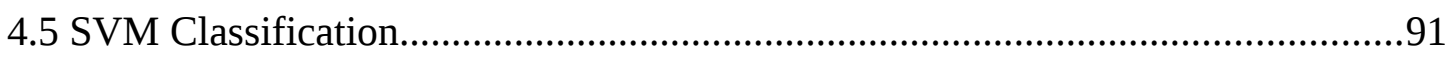

4.5.1 Classification Accuracy Assessment......................................................92

5 DISCUSSION

5.1 Geophysical Basis for Ocean Cover Separability............................................99

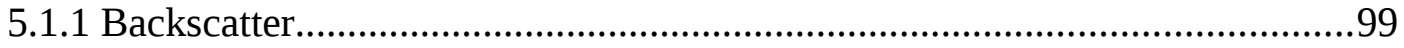

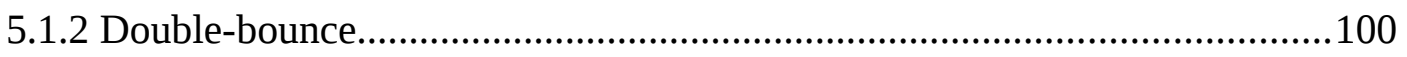

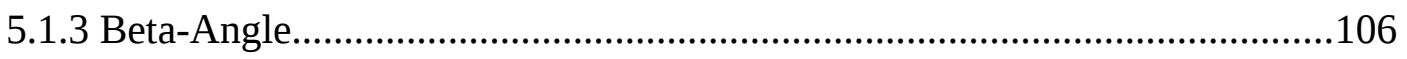


5.1.4 Other SAR Variables. 107

5.2 SAR Variable Selection and Multivariate Gradients using RDA.........................110

5.2.1 Direct Gradient Analysis....................................................................... 111

5.3 Comparison of ANOVA/THSD/G-H and RDA Selection Approaches.................114

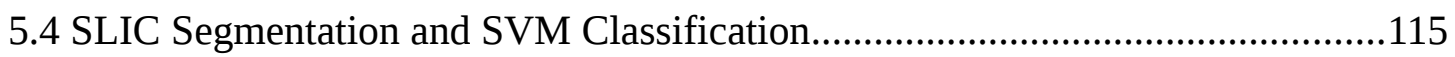

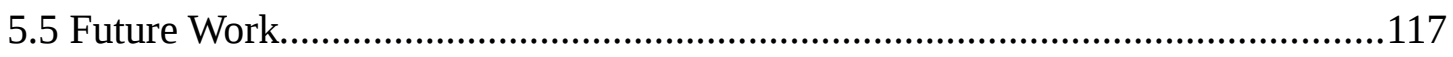

5.5.1 Use of RDA Forward-Selected SAR Variables in an SLIC/SVM Classifier117

5.5.2 Include Feature Formations to Describe Differences in Ice Forms..............117

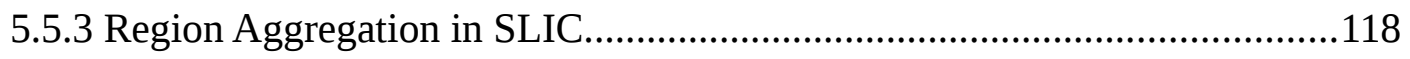

5.5.4 Application of RDA Approach to other Classifications in SAR and Optical

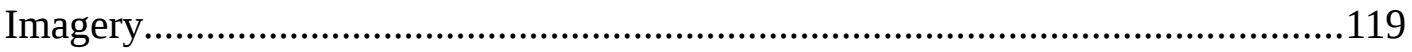

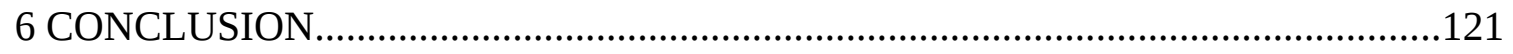

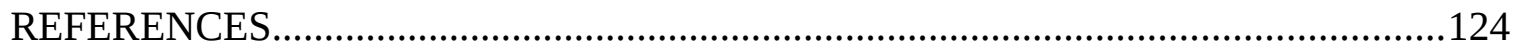

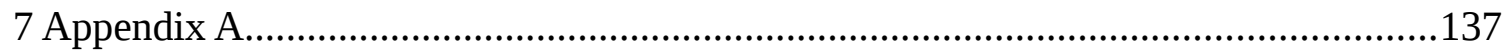

7.1 Derivation of Coherency and Covariance Matrices from the Sinclair Matrix.....137

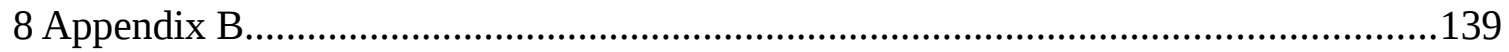

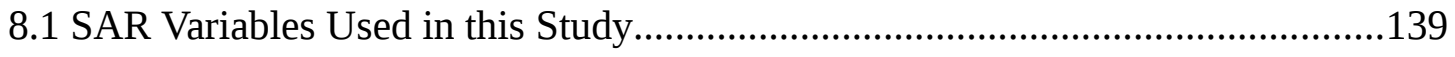

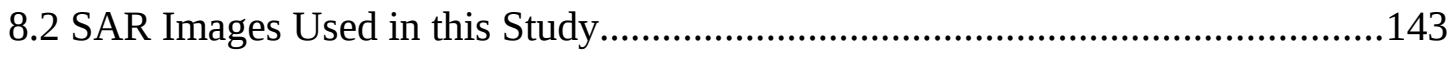

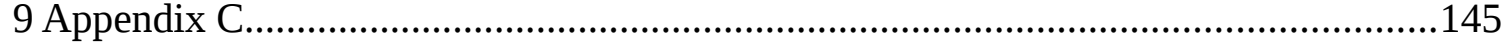

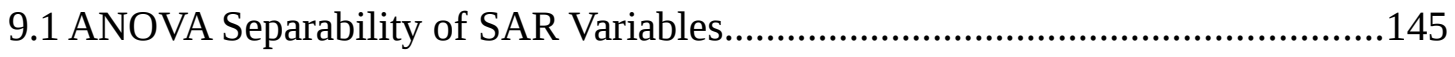

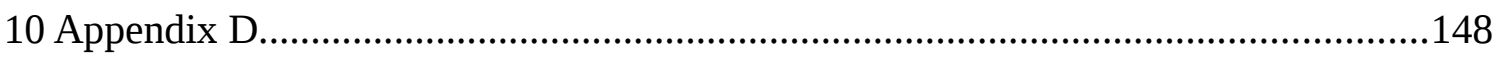

10.1 Assessment of SAR Variables for Separating each Ocean Cover Based Upon

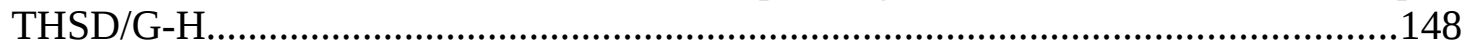

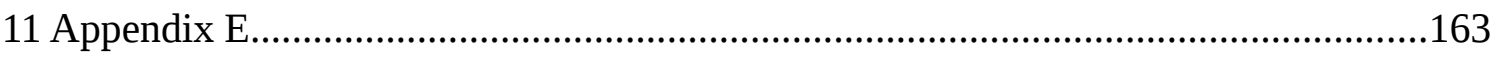

11.1 Accuracy Assessments of the Nine Testing SAR Images................................163 


\section{List of Figures}

Figure 2.1: Common electromagnetic frequencies and wavelengths used in SAR. Letters denote the bands and SAR platforms which use these are noted with their frequency (Richards, 2009). With permission of Springer Science+Business Media.

Figure 2.2: Geometry of radar imaging. The incidence angle $(\theta)$ is the number of degrees from zenith. The range direction is perpendicular to the flight direction while the azimuth direction is parallel. Adapted from Richards, 2009. With permission of Springer Science+Business Media....

Figure 2.3: Different scattering mechanisms of the electromagnetic wave from a SAR sensor with different practical situations where the scattering mechanism could occur (European Space Agency, 2014c).

Figure 2.4: Different interactions between Synthetic Aperture Radar energy and the surface with different textures. A) Smooth (specular) surface, where energy is not returned. B) Intermediate surface. C) Rough surface (Richards, 2009). With permission of Springer Science+Business Media.

Figure 2.5: Bragg scattering over water or tilled agricultural fields where the surface wavelength is a function of electromagnetic wavelength and $\theta$. Constructive interference results when the surface wavelength, radar wavelength and incidence angle reinforce the backscattered signal. Adapted from Shokr and Sinha, 2015.

Figure 2.6: Speckle formation from constructive and destructive interference from the sum of reflected electromagnetic waves. The different overlapping reflections can either form constructive or destructive to varying degrees in a SAR image (Lee and Pottier, 2009).

Figure 2.7: Electric and magnetic field orientation describing the polarization of electromagnetic radiation (Al-Azzawi, 2006).

Figure 2.8: Phase difference between different SAR beams.

Figure 2.9: Typical ice island drift tracks follow primary ocean currents (Crawford, 2013).

Figure 2.10: The common tabular shape of an ice island. This particular fragment was about $20 \mathrm{~m}$ tall and $400 \mathrm{~m}$ wide. Photo courtesy of Andrew Hamilton, 2011..................25

Figure 2.11: Summary of seasonal transition of Synthetic Aperture Radar backscatter for smooth land-fast FYI and MYI for RADARSAT-1 (RSAT-1) HH and ERS-1 and -2 VV (Yackel et al., 2007).

Figure 2.12: $\Phi$ is measured as degrees from incident electromagnetic wave to the surface of the ice island. $0^{\circ}$ is a parallel orientation while $90^{\circ}$ is a perpendicular orientation......31 
Figure 2.13: Ice island appearance in SAR as a Pauli decomposition, red $=\mathrm{HH}+\mathrm{VV}$, green $=2 * \mathrm{HV}$, blue $=\mathrm{HH}-\mathrm{VV}$. This figure shows the characteristic surface ridges on an ice island during summer melt conditions. Pictured are Target 7 (Markham M1 ice island) in the middle, with a smaller ice island fragment to the upper left with marine ice. The surrounding sea ice is darker with less backscatter. FQ3, $6.3 \mathrm{~m}$ pixel size, 2009/07/13 15:27:33 GMT (De Abreu et al., 2011).

Figure 2.14: Changing SAR response due to wind and waves around islands. The pattern of high and low backscatter is consistent with regions that are exposed and sheltered from wind, respectively (Boost-Technologies, 2006).

Figure 2.15: Triplot for RDA axes 1 and 2 ( $\mathrm{x}$ and $\mathrm{y}$ ) with highest forest complexity in lower right and lowest forest complexity in upper left. Red arrows are variables derived from Quickbird imagery or DEM and blue arrows are field measurements. Numbered points are individual field samples (Torontow and King, 2011)...

Figure 2.16: Example of maximum margin linear classification in SVM. D is a superior classification to A, B, C as the overall separation (distance of class members to the class boundary) is maximized to the splitting plane (Improved Outcomes Software, 2014).....45

Figure 2.17: Computation of classifications in one-dimensional space. A simple linearly classifiable dataset with one dimension (a). A more complicated dataset which cannot be classified with a linear kernel (b). Projection of (b) into a higher dimension using some function to allow for a linear classification (c) (Manning et al., 2008)...............................46

Figure 2.18: Computation of classifications in two-dimensional space. Non-linear classifications may be preferable to separate the two groups (a). These classifications, when raised to higher dimensions (b) allow a similar splitting hyperplane. Different classification hyperplanes may exist depending on the kernel used (Wagner, 2012)........46

Figure 2.19: Different kernels for more complex classification splitting hyperplanes. These kernels are different functions which are used to elevate the dataset to higher dimensions (scikit-learn developers, n.d.).

Figure 3.1: Ice island drift tracks, derived from tracking beacons and coverage of RS2 images listed in Table 3.2. The Petermann, Ward Hunt and Markham ice shelves and glacial tongues are also shown.

Figure 3.2: Ice island placement within the parent ice shelves at the northern coast of Ellesmere Island. a) Overview b) Markham Ice Island 1 (M1) in the upper right with Markham Ice Island 3 (M3) in the lower left (2007/01/21), c) Ward Hunt 1 (WH1) (2007/01/24), d) Ward Hunt 2 (WH2) (2008/02/02). All images are RS1. These images were used to orient the surface ridges relative to the overall ice island. Figure adapted from Crawford, 2013.

Figure 3.3: Petermann Glacier calving event in 2010 a) The placement of Petermann ice island fragments within the parent ice island and prior to further fragmentation (2010/08/16). b) The glacier terminus pre-calving (2010/07/21). c) The terminus and ice island post-calving (2010/08/05). Images are from the NASA MODIS Earth Observatory 
(NASA, 2010). These images were used to orient the surface ridges relative to the overall ice island. Figure adapted from Crawford, 2013..................................................................52

Figure 3.4: Example of measuring $\Phi$ for ice island M1. The green line indicates direction of incident SAR beam. The red line is the prevailing ridge direction. The yellow arc is the measured angle. The RS2 image was acquired 2009/11/09. .58

Figure 3.5: Flowchart of analysis methods. The colours indicate the software used........61

Figure 4.1: Histograms of explanatory variables. a) Temperature appeared bimodal b) wind speed was nearly normal c) $\Phi$ occurred with approximately equal frequency d) $\theta$ appeared normally distributed.

Figure 4.2: Boxplots of median backscatter in each image for each ocean cover. a) бoHV b) $\sigma o H V$ c) $\sigma o \mathrm{VV}$. The middle horizontal line represent the median, with the hinges representing the first and third quartiles, and the dots representing outliers.......................76

Figure 4.3: Boxplot of ocean cover median values from each image for the most promising SAR variables at separating specific ocean covers. a) T22 for meteoric ice separability b) Beta2 for marine ice separability c) Krogager_Kd for MYI ice d) Beta for FYI separability e) Neumann_tau for water separability.

Figure 4.4: Correlation matrix of median values for five THSD/G-H and five RDA selected SAR variables. The THSD/G-H SAR variables are in red and the RDA selected SAR variables are in blue. Absolute Spearman correlation coefficients are given. $* * *$ signifies a highly significant correlation of $\mathrm{p} \leq 0.001$. The red line is a fitted function.......82

Figure 4.5: Screeplot of cumulative explained variance of Monte-Carlo forward selected SAR variables.

Figure 4.6: RDA triplot of THSD/G-H selected SAR variables to explain ocean cover identity. The coloured dots represent the different ocean covers in the ordination space. 85

Figure 4.7: RDA triplot of RDA selected SAR variables to explain ocean cover identity. The coloured dots represent the different ocean covers in the ordination space.

Figure 4.8: RDA triplot attempting to explain the RDA selected SAR variables with the confounding factors. The coloured dots represent the different ocean covers in the ordination space.

Figure 4.9: RDA triplot explaining the RDA selected SAR variables with the confounding factors and the ocean cover identity. The coloured dots represent the different ocean covers in the ordination space...

Figure 4.10: RS2 FQ17W image acquired 2012/10/29-21:46:43 $185 \mathrm{~km}$ southeast of Clyde River. a) Pauli decomposition, showing open water and a meteoric ice island fragment. b) SLIC segmentation of the Pauli decomposition where each colour represents a unique object. c) SAR variable classification using Beta, Krogager_Kd, Neumann_tau, Beta2 and T22 for FYI, MYI, water, marine and meteoric ice. Most of the image was correctly classified as water, except for perhaps the small ice island fragments which 
were below the minimum threshold in the SLIC. The ice island however was incorrectly split between MYI and FYI.

Figure 4.11: RS2 FQ15W image acquired of PII-B-1 2012/06/14-21:42:37 145 km southeast of Clyde River. a) Pauli decomposition , showing FYI in different formations and the PII-B-1 meteoric ice island fragment. b) SLIC segmentation of the Pauli decomposition where each colour represents a unique object c) five SAR variable classification using Beta, Krogager_Kd, Neumann_tau, Beta2 and T22 for FYI, MYI, water, marine and meteoric ice. The lead is largely correctly classified, however there is strong confusion on the smooth FYI to the bottom with water and some MYI/FYI confusion on the FYI flow to the right of the image.

Figure 4.12: RS2 FQ19W image acquired 2013/04/08-21:50:38 $90 \mathrm{~km}$ southeast of Home Bay, Baffin Island. a) Pauli decomposition, showing FYI in different formations and an unknown meteoric ice island fragment. b) SLIC segmentation of the Pauli decomposition where each colour represents a unique object c) five SAR variable classification using Beta, Krogager_Kd, Neumann_tau, Beta2 and T22 for FYI, MYI, water, marine and meteoric ice. The FYI is largely correctly classified, however the ice island is not correctly classified as meteoric ice.

Figure 5.1: T22 double-bounce, with edge dihedral reflection towards RS2 from a Petermann Ice Island fragment in open water, symbology to highlight edges. Image acquired 2011/09/15-22:46:52. Blue is weaker double-bounce returns, yellow is medium, and red is strongest 101

Figure 5.2: A T22 RS2 image overlain on a Formosat-2 image of the Ward Hunt Ice Shelf showing the pattern of double bounce scattering. Red represents strong T22 returns while yellow is medium and blue indicates weak T22 returns. The final co-registration was performed manually. RS2 image acquired 2009/03/04-19:07:12. Formosat-2 image acquired 2008/08/28-17:10:27. Formosat-2 image courtesy of Planet Action 2008.......103

Figure 5.3: RS2 scene captured 2010/10/27-14:51:42 and converted to Krogager_Kd. This scene contains MYI, FYI, marine and meteoric and perhaps some small open water leads in the darkest tones. The rubble fields between the MYI floes have the highest Krogager_Kd, while the MYI floes have medium returns slightly less than marine ice. FYI has very low Krogager_Kd returns due to the likely near perfect specular reflection.

Figure 5.4: RS2 image captured on 2011/03/16-23:25:13 and converted to the Beta SAR variable. The MYI, meteoric and marine ice have similar Beta signatures while FYI has a lower signature.....

Figure 5.5: Geometry of FYI (Vant et al., 1978). The randomly oriented c-axes in the upper portions of FYI transition to more horizontally oriented ice crystals in the columnar portion.

Figure 5.6: RS2 scene 2011/09/22-22:42:29 in Navy Board Inlet just west of Bylot Island. The lighter tones are lower Neumann_tau values corresponding to more consistent 
alignment of particles. The darker tones correspond to more random particle orientation. The ice island is to the upper-left, and water is the grey tones through the middle of the image. The darker tones to the bottom are unknown in origin.......................................109 


\section{List of Tables}

Table 2.1: Interpretation of $\mathrm{H}$ values (Massonnet and Souyris, 2008; Richards, 2009)....20

Table 2.2: Scattering mechanism corresponding to values of $\alpha$ (Deng and Wang, 2014). 20

Table 2.3: Theoretical values of $\alpha$ and $\beta$ angles for canonical targets (Yong, 2004).........21

Table 3.1: RS2 operating mode coverage.

Table 3.2: Number of observations of each ice island and ice island type. GRIP report ice island names differ from the names used in this study...... .53

Table 3.3: Summary of all explanatory SAR geometry and environmental variables.......54

Table 3.4: Ocean cover sample sizes. Note that marine and meteoric ice are ice island sub-types .56

Table 3.5: Frequency of CIS chart and RS2 offset. .56

Table 3.6: Weights given to classes during training of SVM models to reduce inherent imbalance of sampling. Weights are from $0 \rightarrow 1$ where a higher weight is given to classes with fewer samples.

Table 4.1: Summary table of backscatter for ocean covers across all SAR. ooVH was not recorded as it is the same as $\sigma 0 \mathrm{HV}$ due to the principal of reciprocity..

Table 4.2: Top SAR variables for separability of specific ocean covers based upon Maximum Base-Level Difference of Means Score with associated THSD and G-H pvalues corrected with FDR.

Table 4.3: Comparing explained variance (\%) for the CCA and RDA ordinations for the four RDA experiments.

Table 4.4: Cumulative amount of variance explained by the 1st, 2nd, 3rd and 4th axes using all SAR variables.

Table 4.5: Cumulative amount of variance explained by the 1st, 2nd, 3rd and 4th axes for the 12 forward-selected SAR variables.

Table 4.6: Cumulative amount of variance explained by the 1st, 2nd, 3rd and 4th axes in the RDA analysis to understand the association of all variables from the Confounding Matrix on the RDA SAR variable matrix...

Table 4.7: Cumulative amount of variance explained by the 1st, 2nd, 3rd and 4th axes in the RDA analysis to understand the relationship of all factors from the combined Confounding Matrix and the Ocean Cover Identity Matrix on the RDA SAR variable matrix

Table 4.8: Cumulative amount of variance explained by the 1st, 2nd, 3rd and 4th axes in 
the RDA analysis to understand the association of all factors from the Confounding Matrix and the Ocean Cover Identity Matrix on the RDA SAR variable matrix but with the effects of the Ice Island Identity matrix accounted for?.

Table 4.9: Accuracy assessment for RADARSAT-2 FQ17W image acquired 2012/10/2921:46:43. Kappa was 0.42...

Table 4.10: Accuracy assessment for RADARSAT-2 FQ15W image acquired 2012/06/14-

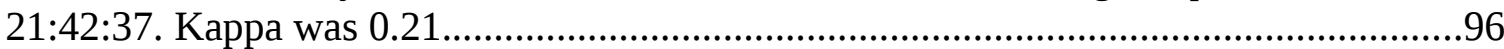

Table 4.11: Accuracy assessment for RADARSAT-2 FQ15W image acquired 2013/04/08-

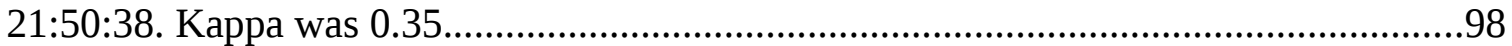

Table 4.12: Overall accuracy assessment for the nine test images. Kappa was 0.38.........98

Table 8.1: SAR variables used in this study..............................................................139

Table 8.2: Confounding variables for each of the training images....................................143

Table 8.3: Test images used for assessing accuracy of SVM/SLIC classification............144

Table 9.1: Measured ANOVA p-values for all 123 SAR variables.....................................145

Table 10.1: SAR variables maximum base-level difference of means scores for separating water from other ocean covers.

Table 10.2: SAR variables maximum base-level difference of means scores for separating FYI from other ocean covers. .151

Table 10.3: SAR variables maximum base-level difference of means scores for separating marine ice from other ocean covers. .154

Table 10.4: SAR variables maximum base-level difference of means scores for separating meteoric ice from other ocean covers........................................................................157

Table 10.5: SAR variables maximum base-level difference of means scores for separating MYI from other ocean covers. 160

Table 11.1: Accuracy assessment of RS2 FQ14 acquired 2010/09/11-23:50:36. Kappa was 0.17

Table 11.2: Accuracy assessment of RS2 FQ6W acquired 2012/06/14-13:28:38. Kappa was -0.07 .

Table 11.3: Accuracy assessment of RS2 FQ17W acquired 2012/10/29-21:46:43. Kappa was 0.42

Table 11.4: Accuracy assessment of RS2 FQ19W acquired 2013/04/08-21:50:38. Kappa was 0.35

Table 11.5: Accuracy assessment of RS2 FQ4W acquired 2013/06/17-11:16:08. Kappa was -0.12

Table 11.6: Accuracy assessment of RS2 FQ24 acquired 2013/06/29-21:58:59. Kappa 
was 0.0 .

Table 11.7: Accuracy assessment of RS2 FQ22 acquired 2013/07/02-22:12:26. Kappa was 0.27 .164

Table 11.8: Accuracy assessment of RS2 FQ15W acquired 2012/06/14-21:42:37. Kappa was 0.21 165

Table 11.9: Accuracy assessment of RS2 FQ13W acquired 2013/03/26-11:36:17. Kappa was 0.01 . 


\section{List of Appendices}

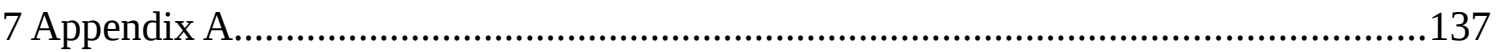

7.1 Derivation of Coherency and Covariance Matrices from the Sinclair Matrix.....137

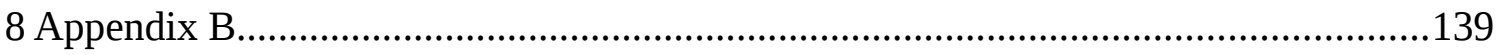

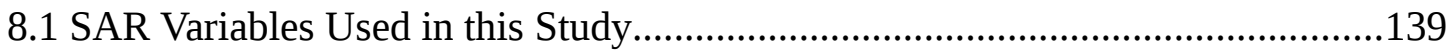

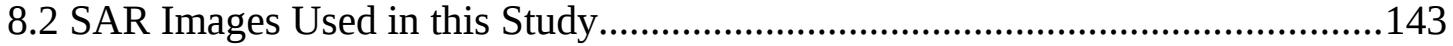

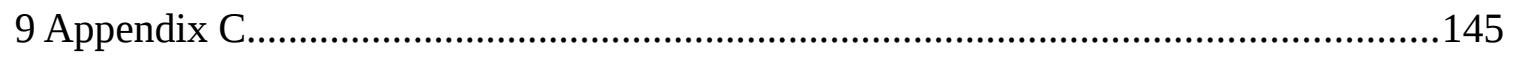

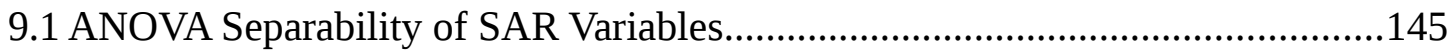

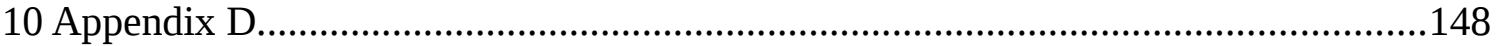

10.1 Assessment of SAR Variables for Separating each Ocean Cover Based Upon

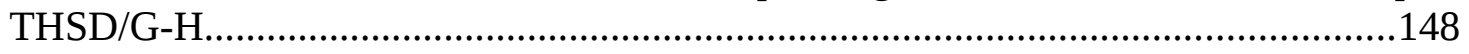

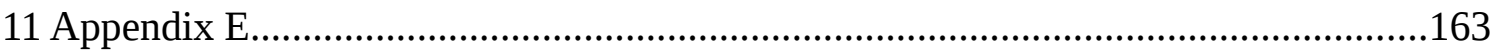

11.1 Accuracy Assessments of the Nine Testing SAR Images..................................163 


\section{List of Abbreviations}

ANCOVA - Analysis of Covariance

ANOVA - Analysis of Variance

CCA - Canonical Correspondence Analysis

CIS - Canadian Ice Service

DEM - Digital Elevation Model

FDR - False Discovery Rate

FQ - Fine-Quad

FQW - Fine-Quad Wide

FYI - first-year ice

GEM-GDPS - Global Environmental Multiscale model-Global Deterministic Prediction

System

G-H - Games-Howell

GIS - Geographic Information Systems

GPS - Global Positioning System

InSAR - Interferometric Synthetic Aperture Radar

MODIS - Moderate Resolution Imaging Spectroradiometer

MRT - Multivariate Regression Trees

MYI - multi-year ice

NDVI - Normalized Difference Vegetation Index

NEST - Next ESA SAR Toolbox

PCA - Principal Component Analysis

RBF - Radial Basis Function

RCM - RADARSAT Constellation Mission

RDA - Redundancy Analysis

RS1 - RADARSAT-1

RS2 - RADARSAT-2

SAR - Synthetic Aperture Radar

SLAR - Side-Looking Airborne Radar

SLIC - Simple Linear Iterative Clustering

SVM - Support Vector Machines

THSD - Tukey's Honest Significant Differences

TLE - Two-Line Element 


\section{INTRODUCTION}

Climate change has been a major driver of change in the Arctic over recent decades. The temperature in the Arctic region has risen at twice the global rate in the past 100 years (Solomon et al., 2007). This substantial temperature increase is due in part to Arctic amplification (Serreze and Barry, 2011). Arctic amplification of atmospheric temperature is caused by positive feedbacks such as the ice albedo feedback in the Arctic climate system. This in turn, has led to an increase in surface air temperatures, furthering the processes that contribute to the feedback (Serreze and Barry, 2011). As a consequence, $75 \%$ of summer sea ice volume in the Northern Hemisphere has been estimated to have been lost since the 1980s (Bindoff et al., 2013). Furthermore, the record minimum sea ice extent in the Arctic was reached in September 2012, when the area covered by sea ice was $49 \%$ less than the 1979-2000 reference period. The oldest, thickest, multi-year ice (MYI) has decreased by 50\% from 2005 to 2012 whereas the proportion of thinner, firstyear ice (FYI) has increased (Bindoff et al., 2013). The sea ice in the Northwest Passage, previously a barrier to historic and modern vessels alike, has diminished in the previous year. This has led to an increase in northern tourism and cargo shipping and has enabled offshore oil exploration and development (Judson, 2010).

Aside from changing sea ice, temperature increases have also been associated with an accelerated period of calving of the ice shelves at the northernmost coastline in the Canadian Arctic (Crawford, 2013). Ice shelves are thick, 5000 year-old ice that are attached to the coast and floating upon the ocean (Jeffries, 2002). Ice shelves are 
composed of freshwater ice which are typically found in protected fjords or bays. Ice shelves can accumulate mass via snowfall on their upper surface, ice accretion on their undersides and also from glacial inflow (White et al., 2015). However, in recent decades there has been almost continual vertical ablation both on their top and bottom surfaces as well as horizontal loss along the seaward and landward edges of the ice shelf (Copland et al., 2007; Crawford, 2013; Mortimer et al., 2012; White et al., 2015). Arctic ice shelves and floating glacial tongues disintegrate into large tabular icebergs termed "ice islands" (Hattersley-Smith, 1962; Koenig et al., 1952). They are on the order of hundreds of metres to tens of kilometres in size with a significant freeboard and ribbed surface (Jeffries, 2002; MANICE, 2005).

Large ice islands were first discovered in the 1940s and have been an ongoing hazard in the Arctic (Koenig et al., 1952). A minimum of 625 various ice island fragments were adrift in 1976 in the southern Beaufort Sea (Spedding, 1977). The northern coast of Ellesmere Island has lost $552 \mathrm{~km}^{2}$ of ice shelf since 2000, with much of this loss is from the calving of the Ayles, Petersen, Wootton, Serson, Markham and Ward Hunt ice shelves (Mueller et al., 2013). More recent calvings from Petermann in 2010 and 2012 have also increased the total ice islands in the Arctic Ocean (Environment Canada, 2013; Falkner et al., 2011). Large ice islands have historically survived as long as a decade or more (Cotell, 1960; Koenig et al., 1952), and fragment into smaller pieces which themselves can be hazards (Crawford, 2013). The increase in marine traffic (Judson, 2010), combined with a higher prevalence of ice hazards such as ice islands (Crawford, 2013), suggests an increased risk of vessel damage and release of hazardous cargo into the 
sensitive Arctic environment.

This has attracted the attention of the Canadian Ice Service (CIS), whose mandate is "to provide the most accurate and timely information about ice in Canada's navigable waters” (Canadian Ice Service, 2015). The CIS fulfills its mandate through the use of remote sensing technology to chart sea ice conditions, as well as monitoring the presence of icebergs and ice islands. Although optical remote sensing has the capability of observing ice and ice islands, optical methods cannot operate during polar night or through cloud cover. The CIS has a long history of charting ice using Synthetic Aperture Radar (SAR) from the RADARSAT-1 (RS1) and RADARSAT-2 (RS2) satellite platforms (Flett et al., 2008). SAR is a suitable remote sensing technology for ice observation since it is an active sensor, providing its own illumination in the microwave band, which is unimpeded by clouds, allowing all-weather, day or night operation (Chan and Koo, 2008). Furthermore, SAR can penetrate many centimetres to metres into the ice surface depending upon salinity, providing some measurement of the nature of the ice itself (Van der Sanden and Drouin, 2011). Further advanced SAR can also use multiple polarizations in the form of dual quad polarimetric imagery. These polarizations (explained in Section 2.1) may be used to further measure the interaction between the SAR microwave energy and the ice, providing enhanced ice detection not available using optical imagery (Shokr and Sinha, 2015).

The CIS has used SAR to find ice islands and track them as they drift around the Arctic Ocean. Within SAR imagery, it has been found that ice and, more specifically, the SAR returns of ice islands are highly variable for unknown reasons (L. Desjardins, pers. 
communication). The appearance of sea ice in SAR is influenced by many factors related to environmental conditions and viewing geometry. Warmer temperatures cause melt conditions and increased water within the ice structure or overlying snow-pack which can greatly reduce SAR backscatter of all ice types (Onstott and Shuchman, 2004). Wind over open water causes high backscatter that can confound the classification of ice versus water (Shokr and Sinha, 2015). Viewing geometry variables such as incidence angle ( $\theta$ ) are known to influence many SAR variables (Gill and Yackel, 2012). Finally, the orientation of ice ridges can also have some effect on the SAR response (Dierking et al., 1994).

This thesis seeks to enhance the understanding of the underlying factors which influence SAR detection of ice, and determine if ice islands can be detected and classified amongst other ocean covers using RS2 polarimetric data. 


\subsection{Objectives}

In order to improve our understanding of ice island detection, the relationship between SAR polarimetric variables, SAR geometry, environmental conditions and ice geophysical properties must be elucidated. This study will examine SAR polarimetric variables in available imagery of ice islands from 2009 to 2014. The ice islands, originating from the northern Ellesmere ice shelves and the floating glacier tongues of northern Greenland, will be identified visually or using satellite tracking beacons. These ice island samples will be compared to background ocean covers, broadly classified as MYI or FYI, along with open water.

The research objectives are to:

1. determine from Fine-Quad (Wide) $(F Q(W))$ scenes which polarimetric SAR variables are best at separating ice islands from the background ocean cover and seek to understand why.

2. determine the effects of SAR geometry and environmental conditions on polarimetric SAR variables.

3. evaluate an unsupervised classification of ice islands, other ice types and open water using polarimetric SAR variables identified in Objective 1.

These objectives will be addressed using $70 \mathrm{FQ}(\mathrm{W})$ mode images that contain one or more ice islands. Ice islands within the SAR scenes as well as other ocean covers will be used to extract samples of SAR variables that will be processed using open source software. The best variables for discriminating ocean covers will be selected by using statistical difference of means tests as well as a multivariate approach (Objective 1). 
Secondly, the relationship between SAR variables and gradients in environmental condition and gradients in SAR geometry will also be examined using a multivariate approach (Objective 2). Finally, using the selected SAR variables, a segmentation and classification algorithm will be used to provide an assessment of practical discrimination of ocean covers within each image (Objective 3).

\subsection{Thesis Structure}

This thesis begins with a literature review in Chapter 2 that introduces concepts for SAR (2.1), ice islands (2.2.1), sea ice (2.2.2), SAR interaction with ice (2.3), and the statistical techniques used in this thesis (2.4).

From this knowledge foundation, the methods used are described in Chapter 3, the results in Chapter 4 and discussion of the broader meaning, significance and limitations

of the research in Chapter 5. The conclusions from the research are presented in Chapter 6.

Appendix A contains equations and other mathematical details on certain SAR matrices. Appendix B contains the list of SAR variables used, as well as the confounding variables for each of the SAR images used in this study. Appendix B also contains a list of the images used in this study. Appendix C contains the results of Analysis of Variance (ANOVA) separability tests and Appendix D contains the results of pair-wise ocean cover separability tests. Appendix E contains the quantitative accuracy assessments for the nine images that were used to test the classifier. 


\section{LITERATURE REVIEW}

\section{1 $\underline{\text { SAR }}$}

\subsubsection{Introduction}

SAR is an active remote sensing technology that images a surface which uses electromagnetic energy in the range of 1-12 GHz. As opposed to passive sensors, SAR transmits and receives its own energy, thus illuminating the surface and providing nearly all-weather capability with little disruption. Many different frequencies have been used in SAR platforms, with X-, C- and L-band centred on 9.7, 5.3 and 1.28 GHz respectively being the most popular (Doerry and Dickey, 2004) (Figure 2.1).

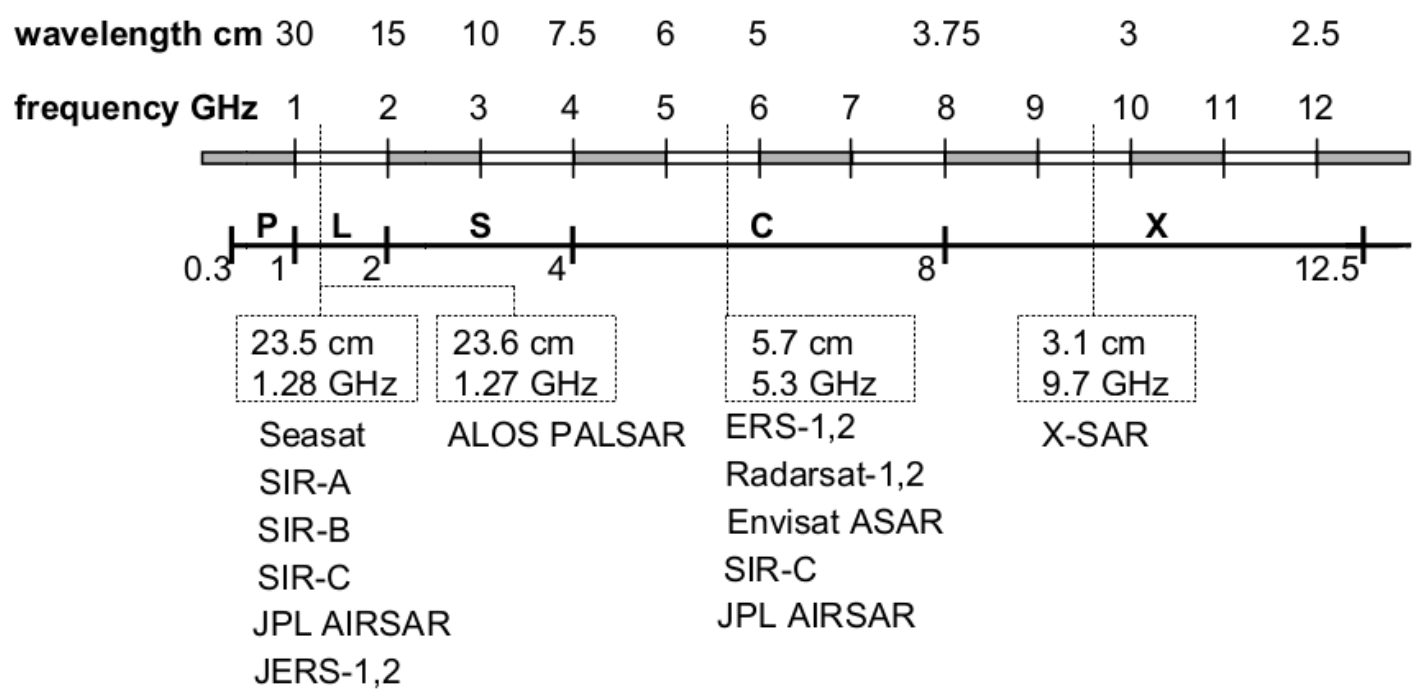

Figure 2.1: Common electromagnetic frequencies and wavelengths used in SAR. Letters denote the bands and SAR platforms which use these are noted with their frequency (Richards, 2009). With permission of Springer Science+Business Media.

Modern SAR systems illuminate with a side-looking antenna (Figure 2.2). Microwaves interact with a large area to one side of the flight track, which becomes the 
final swath width of the SAR image (Richards, 2009). $\theta$ is the degrees from zenith of the incident SAR beam at the surface (Figure 2.2).

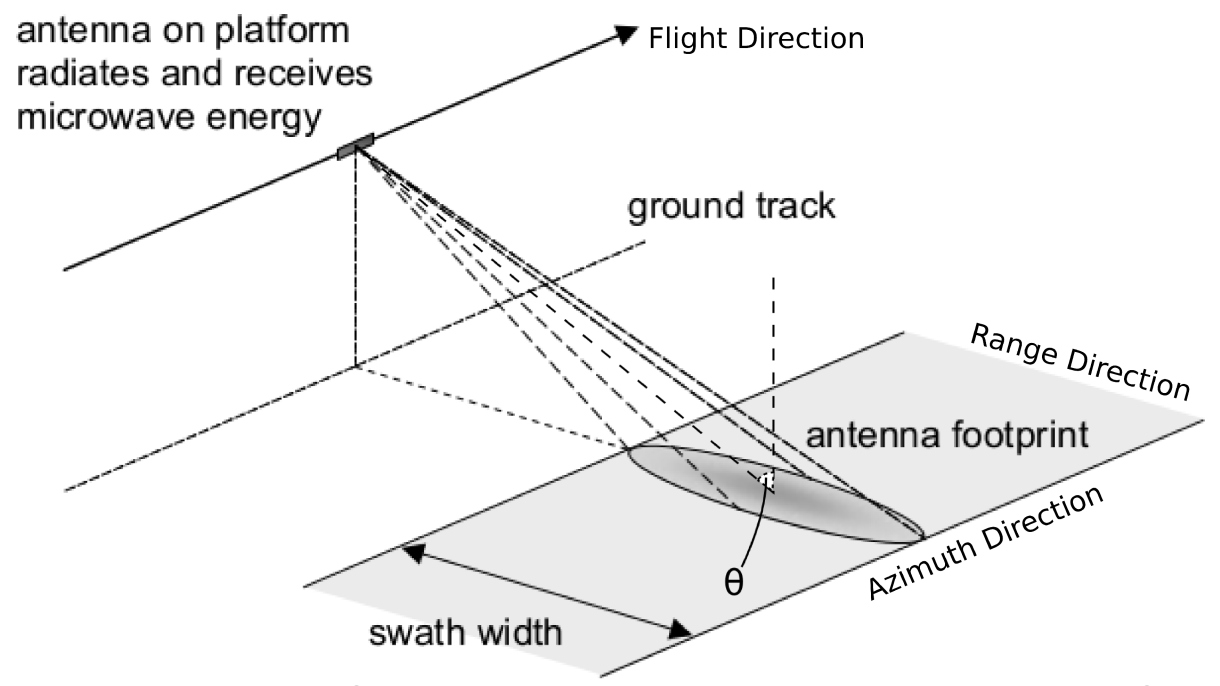

Figure 2.2: Geometry of radar imaging. The incidence angle $(\theta)$ is the number of degrees from zenith. The range direction is perpendicular to the flight direction while the azimuth direction is parallel. Adapted from Richards, 2009. With permission of Springer Science+Business Media.

Single pulses are transmitted by the antenna and the time it takes to reach the surface and return is recorded (Richards, 2009).

At typical pulse length and $\theta$, a radar system would have a coarse range resolution of thousands of metres (Richards, 2009). However, various pulse and radar techniques are employed to enhance the range resolution (Richards, 2009). In the along-track direction, commonly known as the azimuth direction, the resolution is dependent upon the center frequency of the pulse, the range to the target, and the antenna length (Richards, 2009). To achieve an acceptable azimuth resolution in space-borne radar systems, where the range to the target is high, the antenna length would need to be increased to an unmanageable size of thousands of metres (Richards, 2009).

However, an antenna can be 'synthesized' by using platform motion to counter the 
low azimuth resolution of a real antenna (Richards, 2009). Counter-intuitively when using a synthesized antenna aperture, a smaller antenna is more suitable as it leads to a broad beam width which maximizes the duration of target irradiation and thus, the azimuth resolution (Richards, 2009).

\subsubsection{Backscatter}

The interaction between the surface and the microwave pulse will influence the returned energy, also known as backscatter, of the electromagnetic radiation. The amplitude or signal strength of the returned signal is recorded, among other properties (Marino, 2010). The higher the backscatter, the greater the amount of the original

electromagnetic radiation that is returned to the sensor. Depending upon the interaction of the surface, the electromagnetic radiation may behave in different ways. There are three common, primary scattering mechanisms which describe the approximate scattering behaviour of the electromagnetic radiation (Maitra et al., 2013) (Figure 2.3).

1. Surface (odd-bounce) Scattering: interaction occurs principally on the top most surface at discontinuities

2. Double-bounce (even-bounce) Scattering: interaction occurs on the top-most surfaces, off a horizontally and a vertically oriented scatterer

3. Volume (many bounce) Scattering: interaction occurs within the material beneath the surface or within the medium, causing many bounces within that volume 


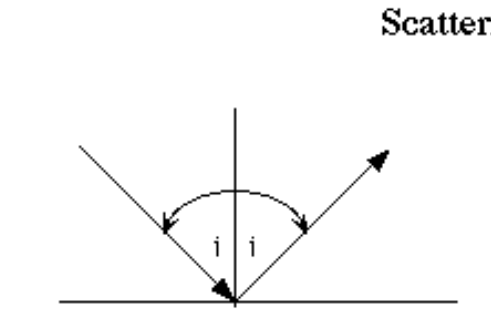

Reflection off a smooth surface The angle of incidence, $i$, equals the angle of reflection.

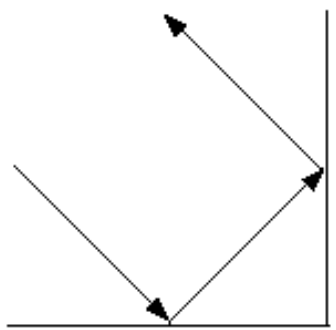

Double Bounce

(Corner Reflector)

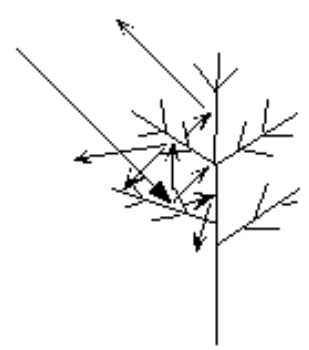

Volumetric Scattering Example scattering in a tree

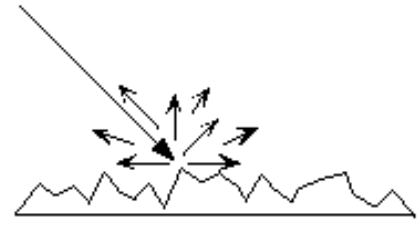

Scattering off a rough surface The variation in surface height is on the order of the incoming signal's wavelength.

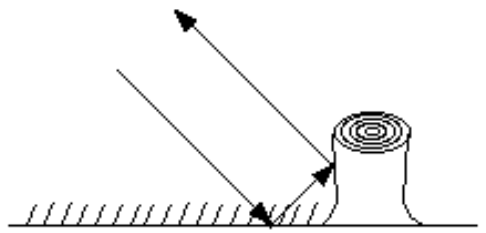

Double Bounce

One possible natural occurence reflecting off two smooth surfaces, grass and a freshly-cut tree's stump

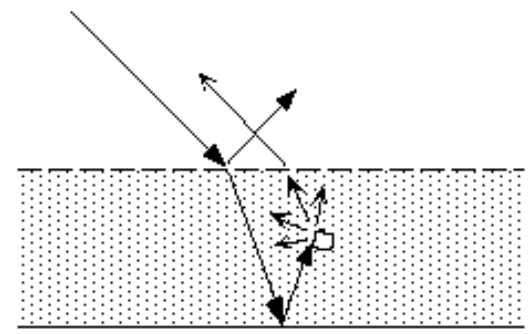

Volumetric Scattering

In this example the incident radiation is both reflected and refracted/transmitted through a layer of dry snow. The refracted radiation then reflects off underlying ice, scatters of a chunk of ice in the snow, and finally refracts back toward the receiver.

Figure 2.3: Different scattering mechanisms of the electromagnetic wave from a SAR sensor with different practical situations where the scattering mechanism could occur (European Space Agency, 2014c).

Textural characteristics of the surface lead to differing backscatter strength (Richards, 2009). Flat surfaces which are not directly oriented towards the sensors will have a very low received signal as most of the pulse will be reflected away (specular reflectance).

Rougher surfaces will have a stronger returned signal as more of the pulse is reflected 
back towards the sensor (Richards, 2009) (Figure 2.4).

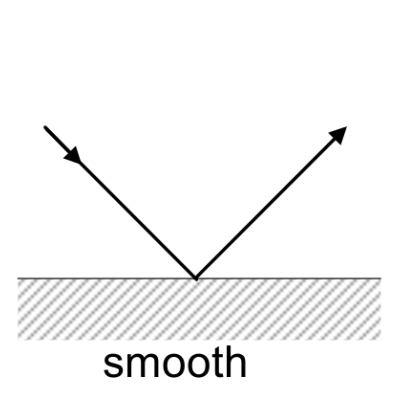

(a)

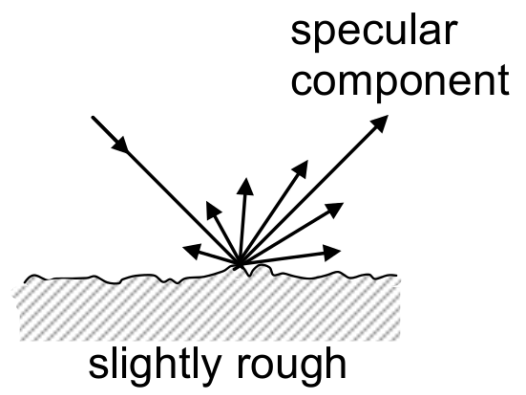

(b)

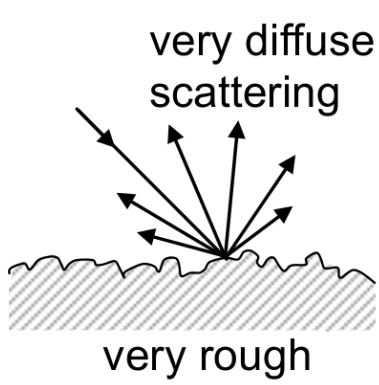

(c)

Figure 2.4: Different interactions between Synthetic Aperture Radar energy and the surface with different textures. A) Smooth (specular) surface, where energy is not returned. B) Intermediate surface. C) Rough surface (Richards, 2009). With permission of Springer Science+Business Media.

For a non-flat surface, backscatter potentially detected by the sensors is a function of wavelength, incidence and the root mean square surface height (h). Commonly, the Rayleigh Criterion is used, but it does not consider medium roughness. The Peake and Oliver Criterion is an extension of the Rayleigh Criterion that also includes intermediate surfaces (Sabins, 2007).

By the Rayleigh Criterion, the boundary between a smooth and a rough surface is $h=\lambda / 8 \sin \theta$. With the Peake and Oliver Criterion, a surface is considered smooth with little or no backscatter to the sensor if $h<\lambda / 25 \sin \theta$. A surface is rough with significant backscatter to the sensor if $h>\lambda / 4.4 \sin \theta$. A surface has a medium roughness with some backscatter and some forward scatter or specular reflectance if $\lambda / 25 \sin \theta<h<\lambda / 4.4 \sin \theta$ where $h$ is the root mean square height $(\mathrm{cm}), \lambda$ is wavelength (cm), and $\theta$ is the incidence angle (Sabins, 2007).

A special form of surface scattering known as Bragg Scattering may occur over water surfaces or agricultural fields in the range direction (Steele et al., 2009). A smooth water 
surface has extremely low backscatter due to specular reflection. As the waves increase, the surface roughness also increases, leading to increasing backscatter. The point at which the backscatter is maximal is known as Bragg Scattering which occurs when the electromagnetic wave is in phase with the surface waves (Shokr and Sinha, 2015). This maximum backscatter occurs when $\lambda_{r}=2 \lambda_{w} \sin \theta$ where $\lambda_{\mathrm{r}}$ is the radar wavelength and $\lambda_{w}$ is the wavelength of surface roughness (for example small water surface waves) and $\theta$ is the incidence angle (Young, 1999) (Figure 2.5). Constructive interference from successive electromagnetic waves reflecting off the ocean surface leads to enhanced backscatter (Young, 1999).

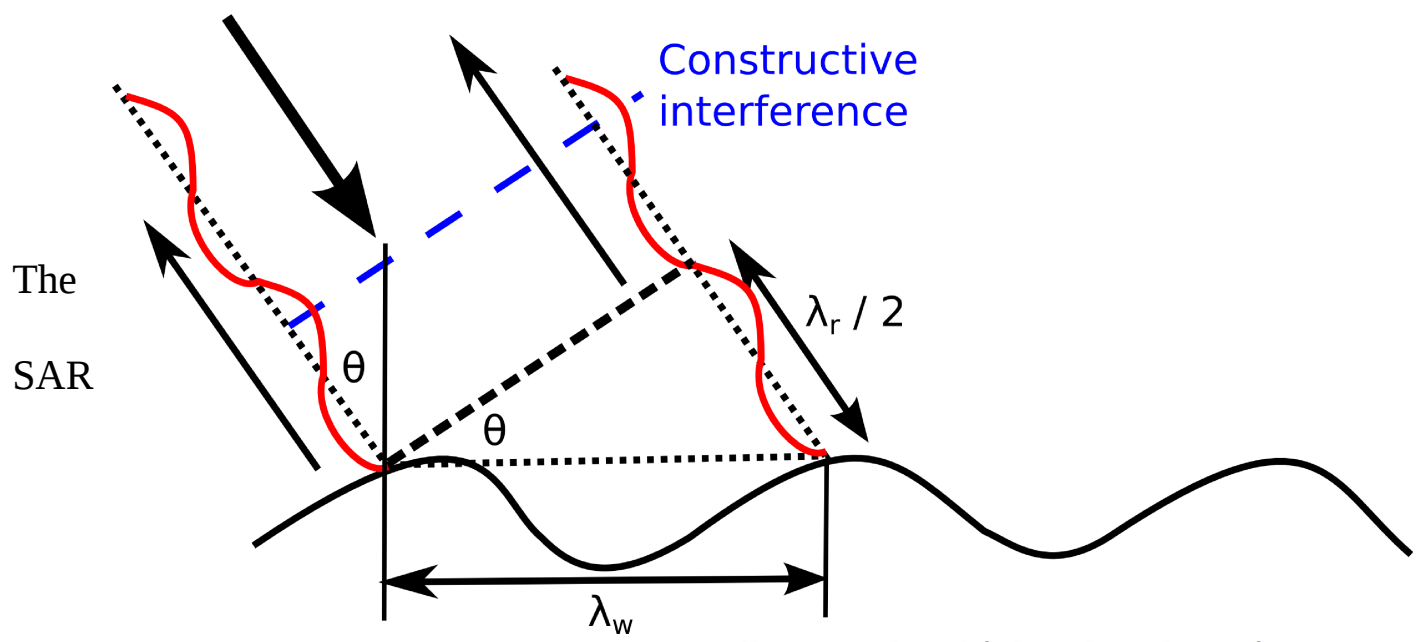

Figure 2.5: Bragg scattering over water or tilled agricultural fields where the surface wavelength is a function of electromagnetic wavelength and $\theta$. Constructive interference results when the surface wavelength, radar wavelength and incidence angle reinforce the backscattered signal. Adapted from Shokr and Sinha, 2015.

wavelength and the electromagnetic properties of the surface material also have an impact on the backscatter. There are three relevant electromagnetic properties: permeability, conductivity and the dielectric constant. The permeability describes magnetic behaviour and conductivity describes the tendency for a material to absorb energy (Richards, 2009), but they are not described further here as they are not as 
important to sea ice backscatter. The dielectric constant or relative permittivity describes to what degree a material can store a charge from an external electromagnetic field such as SAR, and then retransmit that energy (Conyers, 2013). The dielectric constant is a function of salinity, temperature, moisture content, among other characteristics (Onstott and Shuchman, 2004) and is a complex number, describing the amount of electromagnetic radiation which is reflected, absorbed and dissipated (Onstott and Shuchman, 2004). The imaginary component accounts for SAR losses through absorption in the medium (Richards, 2009). As the salinity of ice decreases, the dielectric constant decreases which allows for further penetration of the SAR beam into the ice surface. The dielectric constant itself does not cause a change in backscatter; however, discontinuities between materials with very different dielectric constants will greatly enhance surface backscatter at this boundary (Paradella et al., 2000; Richards, 2009). For example, the interface between differing layers of ice with differing salinities (assuming the same roughness) is a surface scatter since the abrupt change in relative permittivity causes a surface scattering. Volume scattering within for example a tree canopy also contains many permittivity discontinuities between air-twigs and air-leaves but these are not discernible from each other as discrete boundaries (Richards, 2009). Volume scattering may be considered when innumerable single bounce reflections occur within a medium. As the electromagnetic radiation continues to travel through the material, it will be lost by absorption. If the wave is reflected off many internal discontinuities, this represents volume scattering. Longer wavelengths have a further penetration into the volume depending if the dielectric properties are similar (Joseph, 2005). 
Although SAR can yield high spatial resolution and physically describe the surface from its scattering properties, 'noise' is introduced when imaging the surface. This 'noise' is due to the principle of phase of electromagnetic waves.

\subsubsection{Speckle and Speckle Reduction}

Speckle arises in coherent SAR due to constructive and destructive interference of nearby SAR waves that are either in-phase or out of phase with each other due to randomly distributed scatterers (Figure 2.6) (Lee and Pottier, 2009). The returned signal may combine to either form stronger or weaker backscatter in a "salt-and-pepper" pattern (Esch et al., 2010).
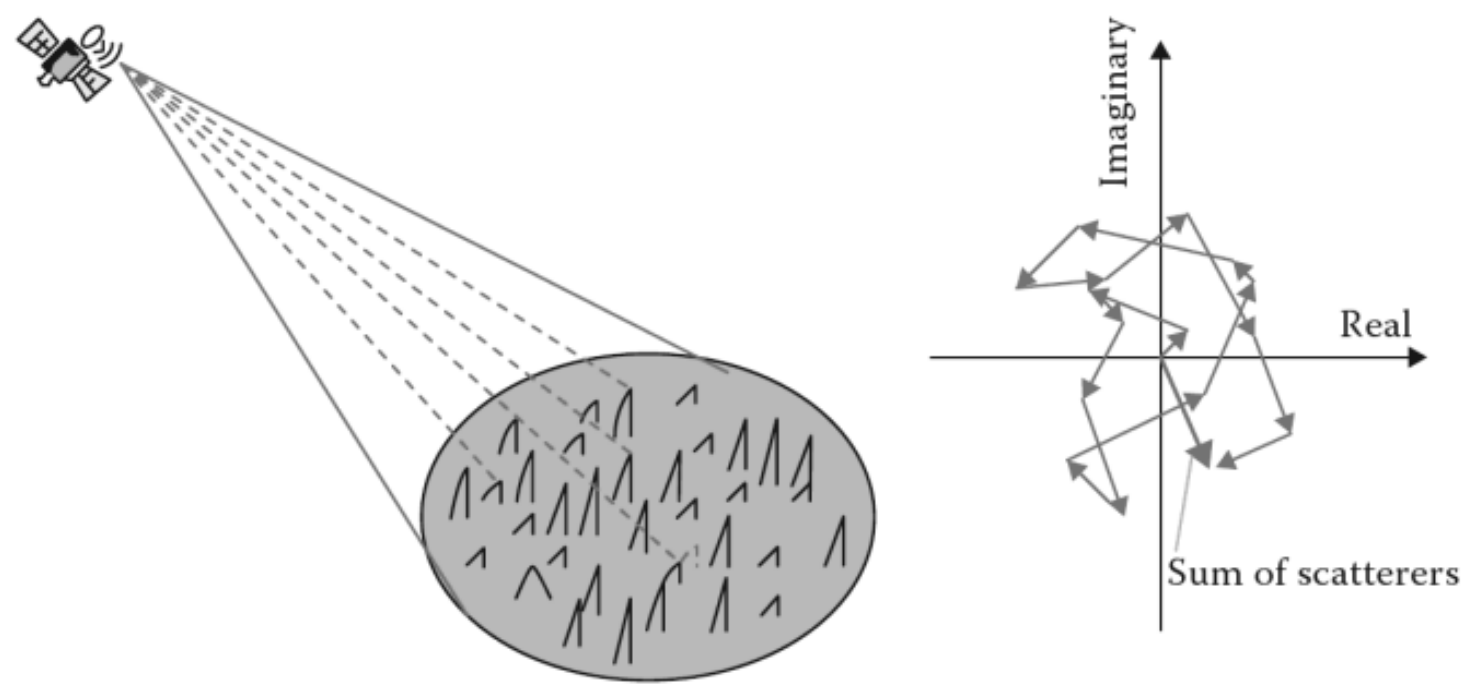

Figure 2.6: Speckle formation from constructive and destructive interference from the sum of reflected electromagnetic waves. The different overlapping reflections can either form constructive or destructive to varying degrees in a SAR image (Lee and Pottier, 2009).

One traditional approach to reducing speckle is to divide the SAR aperture length into segments of individual looks which are considered separately. Each look is processed separately and averaged to form a reduced-speckle SAR image (Lee and Pottier, 2009). However, this approach sacrifices azimuth resolution, which is reduced by a factor of the 
number of looks. Modern single-look complex SAR sensors offset this resolution reduction with an increase in resolution in the azimuth direction during the design process such that the resulting resolution is within specification (Lee and Pottier, 2009).

However, post-acquisition filtering can provide further speckle filtering without the degradation of spatial resolution associated with multilooking. Several filtering algorithms exist to reduce speckle including, Median, Lee, Kuan, Frost and Gamma (Gagnon and Jouan, 1997). Although the speckle filters may perform differently, in general, SAR filters seek to diminish local variability without destroying detail.

\subsubsection{Polarization}

The surface characteristics affect not only the magnitude of the returning electromagnetic wave but also its polarization. The electromagnetic energy that is delivered in the pulse is polarized (Shokr and Sinha, 2015). Electromagnetic energy is formed from perpendicular magnetic and electric fields. These two fields are related by Maxwell's Field Equations and thus, the direction of electric field propagation fully describes the polarization state of the pulse (van Zyl and Kim, 2011) (Figure 2.7). 


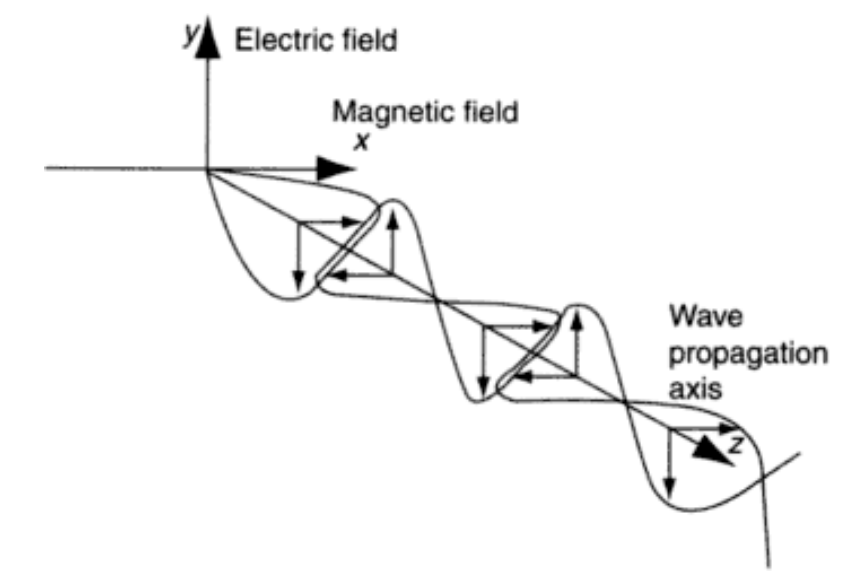

Figure 2.7: Electric and magnetic field orientation describing the polarization of electromagnetic radiation (Al-Azzawi, 2006).

Linear polarizations are the most commonly used with SAR sensors. These polarizations are either horizontal $(\mathrm{H})$ or vertical $(\mathrm{V})$. Since some surfaces can modify the backscattered polarization, both the transmitted and received polarization must be known. This is recorded using "HH", "HV", "VH", and "VV" where the first letter describes the transmitted polarization and the second letter describes the received polarization (Leblon, 2012). Other polarization bases exist such as left- and right-hand circular (Richards, 2009), but these are not discussed here.

The degree of polarization describes to what extent the backscatter is polarized after interaction with the surface (Massonnet and Souyris, 2008). This polarimetric information provides further discrimination as to the geophysical nature of the scattering surface and there are typically considered two primary target types:

1. Coherent targets, which are typical of man-made surfaces with completely polarized returns. Examples are buildings, vessels and aircraft.

2. Incoherent targets, which are natural surfaces with partially polarized returns. 
Examples include ice, water and vegetated surfaces.

These two types of targets have different polarimetric signatures, which are representations of received power depending on incident and received polarization of an electromagnetic wave (Leblon, 2012).

\subsubsection{Phase}

Phase is the relative alignment of the peak in the received electromagnetic wave to the peak in the transmitted wave (Campbell and Wynne, 2011). The greater the misalignment, the greater the phase difference (Figure 2.8). A phase difference occurs due to the distance between the SAR sensor and the reflected surface being a non-integer number of wavelengths. SAR systems record the fraction of the partial wavelength that is received as the phase difference in radians (Pritchard, 2006).

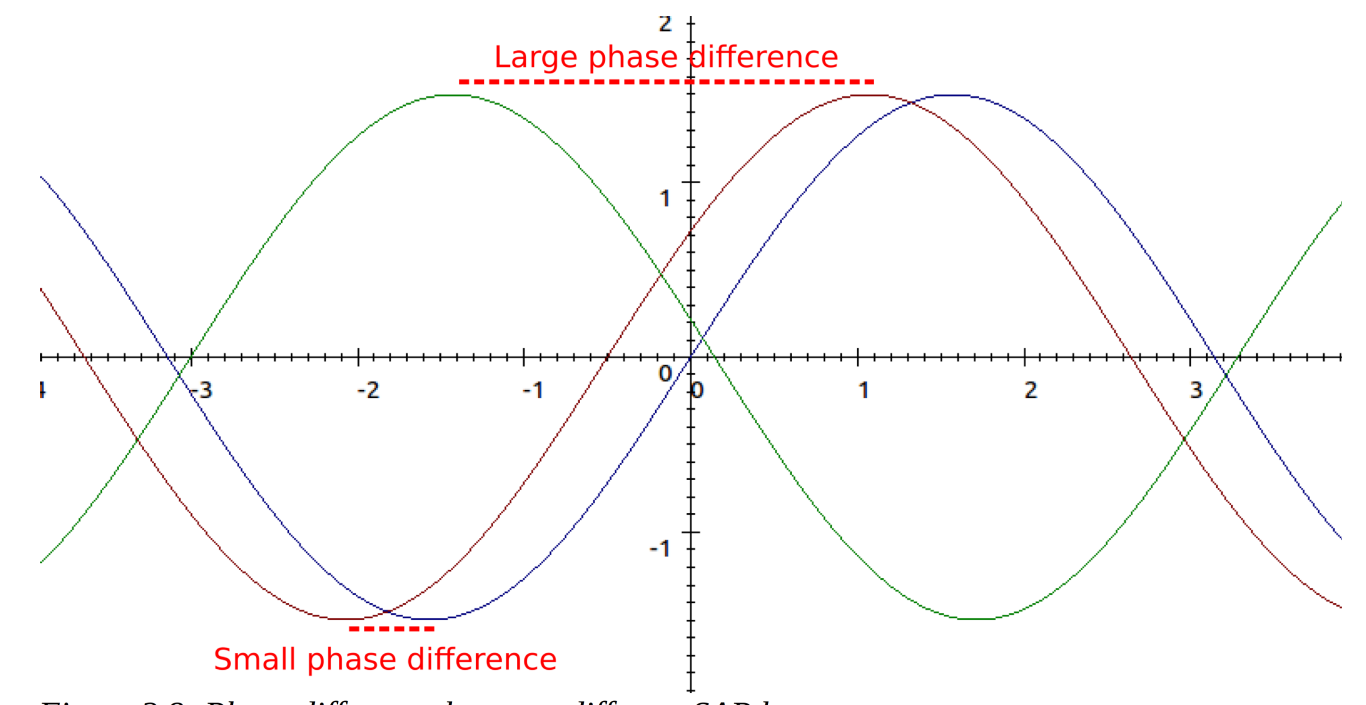

Figure 2.8: Phase difference between different SAR beams.

Alone, the phase does not provide enough information for interpretation of the surface and is expected to look like random noise. In conjunction with other SAR images however, advanced techniques such as Interferometric Synthetic Aperture Radar (InSAR) 
may be used to exploit the relative phase of successive images to extract a Digital Elevation Model (DEM) or determine whether deformation has occurred of the ground (Pritchard, 2006).

\subsubsection{SAR Decompositions and Variables}

From the complex interaction of backscatter, polarization and phase, it is difficult to summarize this multivariate dataset into more easily understood terms with a physical basis for classification. As a result, there have been attempts to summarize this information with various matrices, decompositions (variables describing scattering mechanisms) and polarimetric variables. These polarimetric variables may then directly be used in the classification of surfaces.

\subsubsection{Scattering Matrices}

Due to the multivariate nature of SAR, matrices are used to represent the backscattering in the measured polarizations. The Sinclair or scattering matrix is the most basic representation of backscattering and contains the amplitude and phase measured of all four polarizations HH, HV, VH and VV (Richards, 2009). From this Sinclair matrix, both the coherency and covariance matrices may be determined, which form the basis of many SAR decompositions and variables (Richards, 2009). The coherency and covariance matrices represent the same data and can be considered equivalent and Richards (2009) has further detail about the determination of the coherency and covariance matrices. A brief summary of the covariance and coherency matrices is presented in Appendix A. These matrices and their individual elements can be interpreted 
on the basis of primary scattering mechanisms. For the covariance and coherency matrix, $\mathrm{C}$ and $\mathrm{T}$ respectively, the elements are referred to by their row and column number. The T11, T22, T33 diagonal elements of the coherency matrix refer to single-bounce, doublebounce and volume scattering respectively (Betbeder et al., 2014; Ma et al., 2013). The covariance matrix elements themselves are not as relatable to primary scattering mechanisms as coherency matrix elements, but both matrices are used in deriving SAR incoherent decompositions as briefly described in Section 2.1.5.3.2.

\subsubsection{SAR Variables}

From the scattering matrices, there are numerous polarimetric variables which attempt to quantify some characteristic of the SAR backscatter. Although an individual polarimetric variable may not provide the ability to fully classify a surface, multiple SAR variables together may provide the ability to classify a scene. Many of these variables are derived from the eigenvalues or eigenvectors of the various scattering matrices. Variables such as alpha, entropy, and anisotropy describe the full primary, secondary and tertiary scattering mechanisms of a surface. The beta-angle describes the orientation of the target scatterer. Richards (2009) has further detail about the derivation and meaning of the various polarimetric variables. Below some of the more common or more useful SAR variables in this study are briefly described.

Entropy (H) ranges from 0 to 1 (Table 2.1) and describes the relative importance of the scattering mechanisms without identifying which are more important. 
Table 2.1: Interpretation of H values (Massonnet and Souyris, 2008; Richards, 2009).

\begin{tabular}{cc}
\hline $\mathbf{H}$ & Scattering Description \\
\hline $\mathrm{H}=0$ & Pure coherent scattering target. Backscatter is fully polarized \\
$\mathrm{H}=1$ & All three scattering mechanisms have equal contribution with no dominant scattering. \\
Backscatter is fully unpolarized
\end{tabular}

Alpha $(\alpha)$ ranges from $0^{\circ}$ to $90^{\circ}$ and describes the average dominant scattering mechanism (Table 2.2).

Table 2.2: Scattering mechanism corresponding to values of $\alpha$ (Deng and Wang, 2014).

\begin{tabular}{cc}
\hline Scattering Mechanism & $\boldsymbol{\alpha}$ \\
\hline Single-bounce & $0^{\circ}$ \\
Volume & $45^{\circ}$ \\
Double-bounce & $90^{\circ}$ \\
\hline
\end{tabular}

Anisotropy (A) ranges from 0 to 1 and describes the amount of mixing or relative importance of the second and third most important scattering mechanisms. If A is high, the second scattering mechanism has high relative importance to the third scattering mechanism. If $\mathrm{A}$ is low, the second and third scattering mechanisms have the same importance (Lee and Pottier, 2009; Richards, 2009). A is determined based upon the difference of the two minor eigenvalues: $A=\left(\lambda_{2}-\lambda_{3}\right) /\left(\lambda_{2}+\lambda_{3}\right)$ where $\lambda$ is the second or third eigenvalue depending on the subscript (Richards, 2009). A removes some of the ambiguity of $\mathrm{H}$, which does not describe the complete scattering profile of the surface. A is used in combination with $\mathrm{H}$ and $\alpha$ to describe the full scattering characteristics of a surface (Cloude et al., 2002). H/A/ $\alpha$ are also used in the later discussed Cloude-Pottier decomposition where these three variables are partitioned into classes according to their scattering characteristics (Richards, 2009). 
Beta ( $\beta$ ) ranges between $-\pi$ and $\pi$ radians and provides a description of the orientation of the target scatterer around the radar's line-of-sight (Iwashita and Qong, 2006; Mansouri et al., 2010; Turner and Woodhouse, 2012). $\beta$ is commonly related to the angle of terrain slopes (Leblon, 2012; Touzi, 2007). $\beta$ may be extracted from the eigenvectors of the coherency matrix (Mansouri et al., 2010). There are three $\beta$ angles $\left(\beta_{1-3}\right)$. The best estimation of $\beta$ comes from a weighted average of: $\beta=P_{1} \beta_{1}+P_{2} \beta_{2}+P_{3} \beta_{3}$ where $P_{n}$ is the probability of eigenvector $\lambda_{n}$ is given by $P_{n}=\lambda_{n} / \sum_{m=1}^{3} \lambda_{m}$ (Praks, 2012). Table 2.3 describes how $\beta$ relates to common scattering targets.

Table 2.3: Theoretical values of $\alpha$ and $\beta$ angles for canonical targets (Yong, 2004)

\begin{tabular}{ccc}
\hline Canonical Scatterer & $\boldsymbol{\alpha}$ & $\boldsymbol{\beta}$ \\
\hline Sphere & $0^{\circ}$ & $-180^{\circ}$ to $180^{\circ}$ \\
Dihedral at $\theta$ & $90^{\circ}$ & $2 \theta$ \\
Dipole at $\theta$ & $45^{\circ}$ & $2 \theta$ \\
Helix & $90^{\circ}$ & $\pm 45^{\circ}$ \\
Surface at $\theta$ & $0^{\circ}$ & $2 \theta$ \\
\hline
\end{tabular}

\subsubsection{3 $\underline{\text { SAR Decompositions }}$}

The purpose of decompositions is to simplify the scattering matrix into simpler components, relating to the real scattering mechanisms or physical properties. Many of the SAR decompositions overlap in the scattering mechanisms they describe but differ in the mathematics to achieve the result. The description of SAR decompositions below is provided very generally and only for some of the more common and useful decompositions in this study. 


\subsection{Coherent}

Coherent decompositions attempt to represent a target as pure, primary scattering mechanisms (Massonnet and Souyris, 2008) and are intended for these types of targets. Coherent decompositions use the Sinclair scattering matrix as the basis for the decomposition. The Krogager decomposition was used in this thesis as it was found to aid in ocean cover separability and is briefly described here.

The Krogager decomposition decomposes the SAR response into a combination of a model of a sphere, a diplane and a helix. $K_{s}, K_{d}$ and $K_{h}$ represent the amplitude contribution of each of these scattering types to the Sinclair matrix (Krogager, 1990).

\subsection{Incoherent}

Since coherent decompositions cannot properly describe distributed, non pure scatterers, incoherent decompositions have been derived in order to simplify the complex scattering process, and attempt to decompose multiple, combined scattering mechanisms (Massonnet and Souyris, 2008). These SAR decompositions use the coherency and covariance matrices. The Freeman-Durden, Neumann, Van Zyl, Cloude-Pottier, Pauli and Touzi decompositions were used in this study and many were found to be useful for

separability of ocean covers. The Freeman-Durden decomposition represents the covariance matrix as the combination of the volume, double-bounce and single-bounce scattering mechanisms (Freeman and Durden, 1998).

The Neumann decomposition is a vegetation model using polarimetric covariance and coherency matrix elements to describe various characteristics of the vegetation and particle shapes. $\delta$ describes the effective particle shape. $\Psi$ describes the particle 
orientations in the polarization plane. $\tau$ describes the randomness of the orientation of the particles in the polarization plane (Neumann et al., 2009). As $\tau$ approaches 0, the particles are strongly aligned in a particular direction while as $\tau$ approaches 1 , the particles are randomly oriented.

The Van Zyl decomposition differs from other SAR decompositions in that it ensures that all covariance matrices from the decomposition will have non-negative eigenvalues. Negative eigenvalues are a non-physical result (such as negative backscatter power) in SAR which can occur with the Freeman-Durden decomposition, amongst others (van Zyl et al., 2011). There are three types of scattering mechanisms represented by the Van Zyl decomposition, volume, double-bounce and single-bounce scattering.

The Cloude-Pottier decomposition is based on the previously mentioned H/A/ $\alpha$ variables and is an attempt to combine these three SAR variables into a 3-dimensional space which is then partitioned into sixteen possible classes where each has a unique set of scattering mechanisms. This partitioning may be combined with a minimum distance classifier to form an unsupervised classifier (Lee et al., 1999).

The Pauli decomposition is yet another way to display SAR data, and assigns the elements of the coherency matrix, | HH-VV |, | 2HV | and | HH+VV |, to the red, green and blue channels respectively (Marino, 2010). | HH+VV | represents single- or oddbounce scattering, typically from single specular surfaces, e.g.: water. | HH-VV | represents double- or even-bounce scattering, e.g.: building and ground interactions. | 2HV | represents volume scatterers which return the inverse polarization to the transmitted one, e.g.: forest (Marino, 2010; Zhang et al., 2009). 
The Touzi decomposition attempts to represent both coherent and incoherent target as SAR variables which are invariant to the orientation angle of the target (Touzi, 2007). For incoherent target decomposition, six SAR variables are described for each eigenvector (i) which represents the eigenvalue of the scattering mechanisms $\left(\lambda_{\mathrm{i}}\right)$. These include the Kennaugh-Huynen maximum amplitude $\left(\mathrm{m}_{\mathrm{i}}\right)$, Kennaugh-Huynen orientation angle $\left(\psi_{\mathrm{i}}\right)$, Kennaugh-Huynen helicity $\left(\tau_{\mathrm{mi}}\right)$, the symmetric scattering magnitude $\left(\alpha_{\mathrm{si}}\right)$ and the symmetric scattering type phase ( $\left.\Phi_{\text {asi }}\right)$ (Touzi, 2007). $\lambda_{\mathrm{i}}$ and $\mathrm{m}_{\mathrm{i}}$ were not included in this study.

\subsection{Ice Islands and Sea Ice}

\subsubsection{Ice Islands}

\subsubsection{Characteristics}

Ice islands are large tabular icebergs that calve from thick ice shelves and floating glacier tongues that have increased in prevalence in Arctic waters over the last decade (Crawford, 2013). There are two major source areas for Arctic ice islands: ice shelves along the northern coast of Ellesmere Island and the large outlet glaciers of NW Greenland. Ice islands from northern Ellesmere ice shelves are transported southwest along the northern limit of the Canadian Arctic Archipelago and can be recirculated in the Beaufort Gyre (Figure 2.9). Northern Greenland ice islands are transported south through Nares Strait into Baffin Bay, and eventually carried south via the Labrador Current (Figure 2.9). 


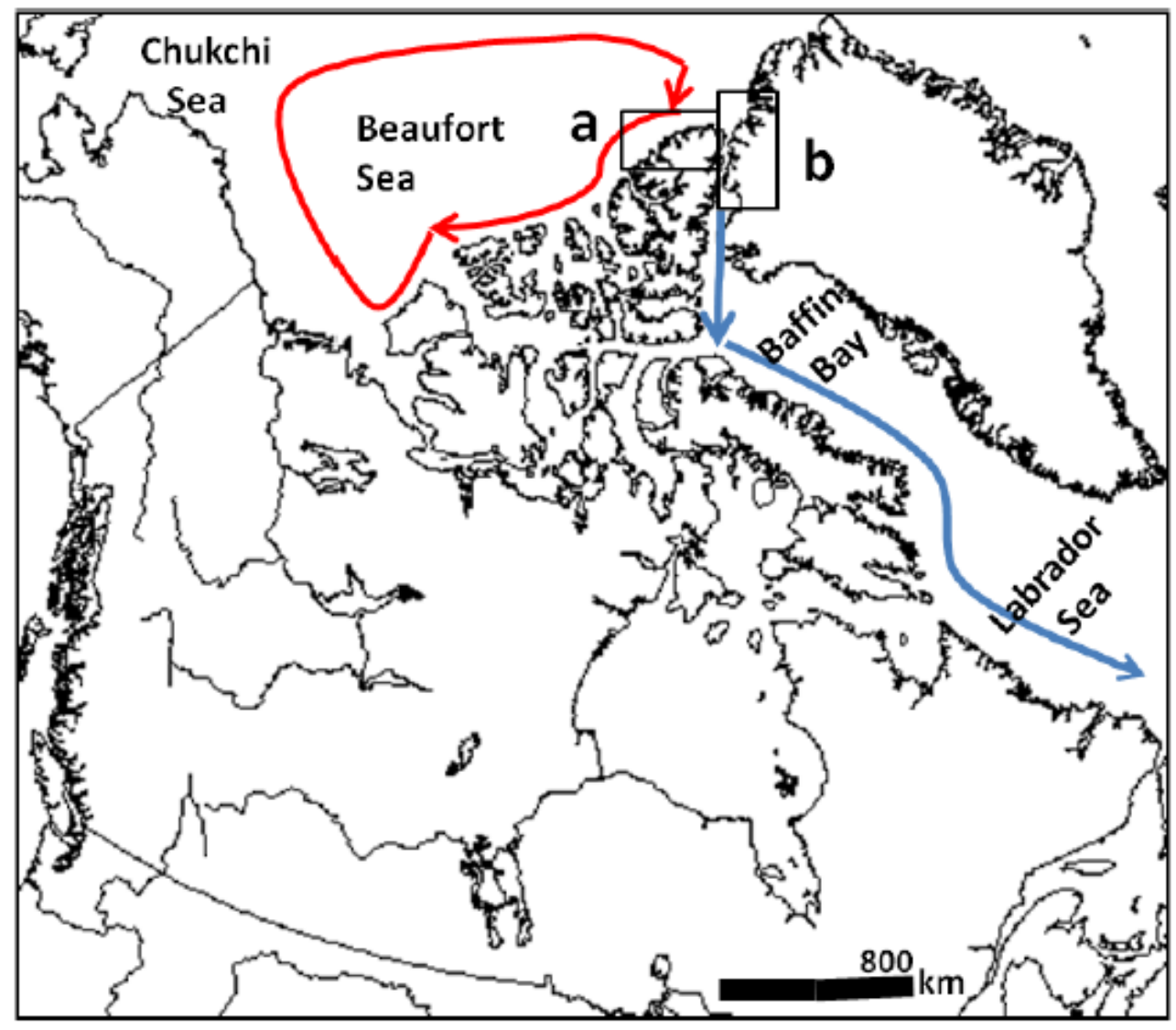

Figure 2.9: Typical ice island drift tracks follow primary ocean currents (Crawford, 2013).

The main distinguishing feature between 'typical' icebergs and ice islands is size and shape. Ice islands are significant ice features and, in exceptional cases, they can be up to $500 \mathrm{~km}^{2}$ in area (Jeffries and Sackinger, 1990). Furthermore, ice islands are tabular in shape, retaining the characteristics of the parent ice shelf (Figure 2.10).

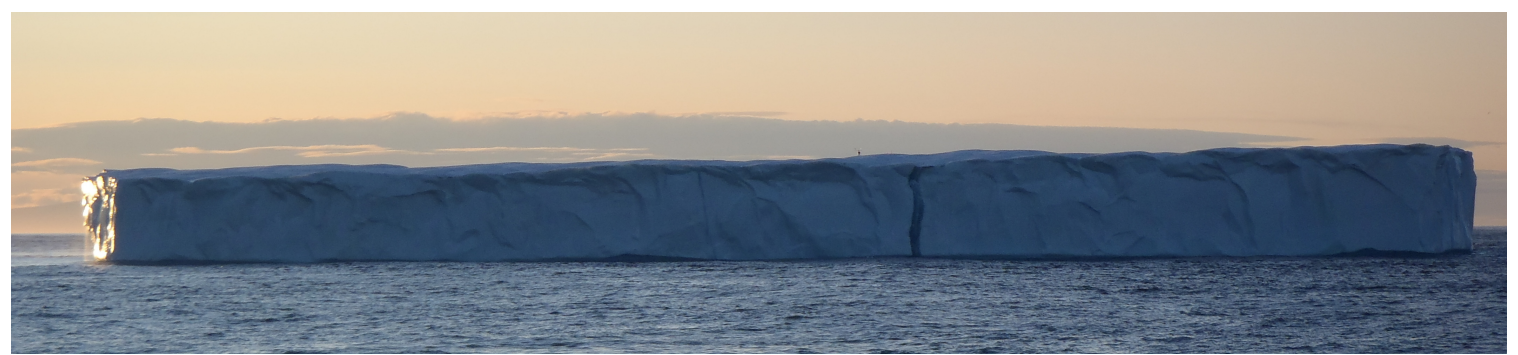

Figure 2.10: The common tabular shape of an ice island. This particular fragment was about $20 \mathrm{~m}$ tall and $400 \mathrm{~m}$ wide. Photo courtesy of Andrew Hamilton, 2011. 
There is no lower limit to ice island size, but it could be considered to be approximately 300 - $400 \mathrm{~m}$ in length, when separation from icebergs becomes difficult (Jeffries and Sackinger, 1990). An ice island is commonly covered with surface ridges and troughs which is typical of the Ellesmere ice shelves (Hattersley-Smith, 1957; Holdsworth, 1987); however, this is less pronounced in Greenlandic ice islands.

Ice islands are considered a hazard to maritime navigation and proposed off-shore platforms and associated infrastructure such as wellheads and undersea pipelines (Fuglem and Jordaan, In Press.). Various countermeasures have been proposed for managing ice island hazards. Mellor et al. (1977) concluded that blasting was ineffective and proposed using thermite or drilling shotholes within the ice structure for explosives. Other countermeasure ideas include skirting infrastructure with sloping shields to induce fracturing of ice islands, creating ice rubble barriers artificially to absorb impact, and pumping seawater onto the surface of ice islands, to accelerate melt at structural weakpoints (Sackinger et al., 1991). Very few of these concepts have been tested and, regardless, a complete ice management strategy must include an early warning mechanism such as detection of hazards with remote sensing (Fissel et al., 2011).

\subsubsection{Ice Island Ice Types}

Ice shelves, in particular those along the northern coast of Ellesmere Island, are composed of various ice types (Marshall, 1955). Some of these ice types originate from sea water while others originate from terrestrial ice (Marshall, 1955). Ice islands often have both a marine and meteoric ice component depending upon their original location in the ice shelf. Marine ice is of seawater or brackish water origin and has a higher bulk 
salinity than meteoric ice, which originates from freshwater rain and snow that accumulates in-situ or flows in from glaciers. These marine and meteoric ice island components are discernible from each other in SAR imagery (De Abreu et al., 2011; Richer-McCallum, 2015) and yet can easily be confounded with FYI and MYI, respectively (De Abreu et al., 2011).

\subsubsection{Sea Ice}

Ice islands adrift in the Arctic Ocean are often surrounded by sea ice of varying age, formation and thickness. Each of these sea ice characteristics leads to differing appearance in SAR. The following section briefly reviews the stages of ice development that are relevant to this study.

\subsubsection{Sea Ice Types and General SAR Response}

Sea ice typically forms when sea water reaches its freezing point of $-1.8^{\circ} \mathrm{C}$ and forms 'new ice'. It then passes through the nilas phase (up to $10 \mathrm{~cm}$ thick) and then to young ice (up to $30 \mathrm{~cm}$ thick). Ice that is thicker than young ice is termed FYI and is greater than 30 cm in thickness (MANICE, 2005). Any FYI that survives a summer's melt is termed old ice and is known as either second year ice or MYI (MANICE, 2005). These two ice types are distinct in age, leading to differences in salinity and thus, SAR returns (Onstott, 1992).

FYI is freshly frozen, and has higher salinity than MYI. This increased salinity minimizes SAR penetration depth, and if it is undeformed, leads to a single-bounce, near perfect specular surface which produces low backscatter. The penetration depth of FYI is 
limited since the salinity absorbs much of the incident electromagnetic energy due to the high dielectric constant (Onstott and Shuchman, 2004; Similä et al., 2010).

The salinity, and thus the dielectric constant, of the ice matrix can vary significantly with the freezing and melting process. During freezing, much of the brine is expelled out of the ice pack, but some remains, increasing the backscatter in SAR (Onstott and Shuchman, 2004). As the ice floe thaws, the brine is flushed to the ocean, leading to further desalination and changing the SAR appearance once again (Yackel et al., 2007). Due to this change in the brine content of the ice, MYI and FYI can have a very different SAR appearance during optimal viewing conditions in the winter. In general, MYI has higher backscatter than FYI but this changes significantly during transition phases (Yackel et al., 2007) where water content increases during melt, leading to enhanced backscatter due to changing dielectric constant which enhances both reflection as well as internal loss through absorption in the medium (Richards, 2009) (Figure 2.11).

Both FYI and MYI can be deformed into ridges and rubble fields by convergent motion. In this case, double-bounce scattering is possible and backscatter is increased (Hossain et al., 2014). The surface of MYI is typically more variable than FYI due to ridges and features from previous summers' melt (Johnston and Timco, 2008). 


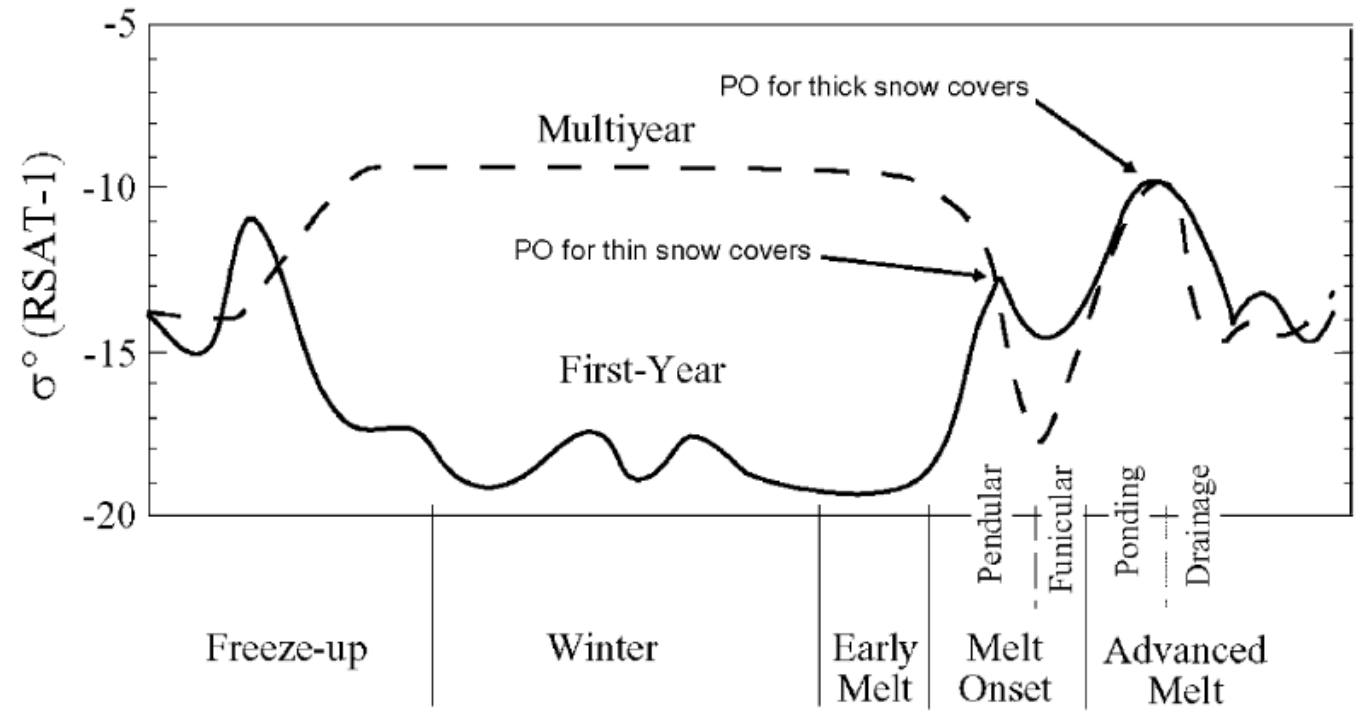

Figure 2.11: Summary of seasonal transition of Synthetic Aperture Radar backscatter for smooth land-fast FYI and MYI for RADARSAT-1 (RSAT-1) HH and ERS-1 and -2 VV (Yackel et al., 2007).

\subsubsection{Ice Charts}

The CIS releases regional ice charts (MANICE, 2005) that map ice conditions across the Canadian Arctic, as well as the Great Lakes (Shokr and Sinha, 2015). These ice charts are a culmination of analysis from multiple sources, including visual interpretation from the ship or aircraft and/or imagery including optical, infrared, Side-Looking Airborne Radar (SLAR), SAR or passive microwave by trained ice analysts (MANICE, 2005). These ice charts are quality controlled and are available in digital formats, enabling easier integration into Geographic Information Systems (GIS) workflow. Regional charts are

produced for the Canadian Arctic (Eastern, Western and Hudson Bay), Great Lakes and East Coast of Canada and are primarily intended for climatological records.

The CIS classifies ice type and cover in a systematic way. These ice conditions are summarized in an egg code which denotes the total ice concentration, partial concentration of ice types, stage of ice development and the form of the ice (MANICE, 
2005). The egg code classification is not fully described here, but is available in MANICE (2005). The total ice concentration describes the total coverage of ice within the classified region. The partial concentration describes the total coverage of various ice types within each classified region. Stage of ice development describes the ice type, generally including estimated age and thickness. The form of the ice describes the

physical nature of the ice and how the ice has been physically reformed into small, medium, and large floes.

\subsection{SAR Interaction with Ocean Covers and Classification}

\subsubsection{Ice Islands}

The surface ridges on ice islands have been found to have unique characteristics in SAR. Depending on the azimuth angle $(\Phi)$ of the electromagnetic wave to the surface ridges, it was found that in airborne X-band STAR-2 HH imagery of a range resolution of $16 \mathrm{~m}$ and azimuth resolution of $6 \mathrm{~m}$, the ribbed surface texture could vary from very bright, light-grey tones to lesser contrast, middle-grey tones (Jeffries and Sackinger, 1990). The best contrast was when the SAR antenna was perpendicular to the surface ridges (Figure 2.12). Jeffries and Sackinger (1990) also noted that ice island detection would have been extremely difficulty without the aid of a priori location knowledge from tracking beacons.

Dierking et al. (1994) imaged an ice island with SEASAT-A L-band HH, CV-580 Lband $\mathrm{HH}$ and $\mathrm{HV}$, and $\mathrm{X}$-band $\mathrm{HH}$ polarizations. Much like Jeffries and Sackinger (1990), it was found that the most evident features were surface ridges and they were similarly found to be the most evident when the view azimuth was perpendicular to the 
ridges (Dierking et al., 1994).

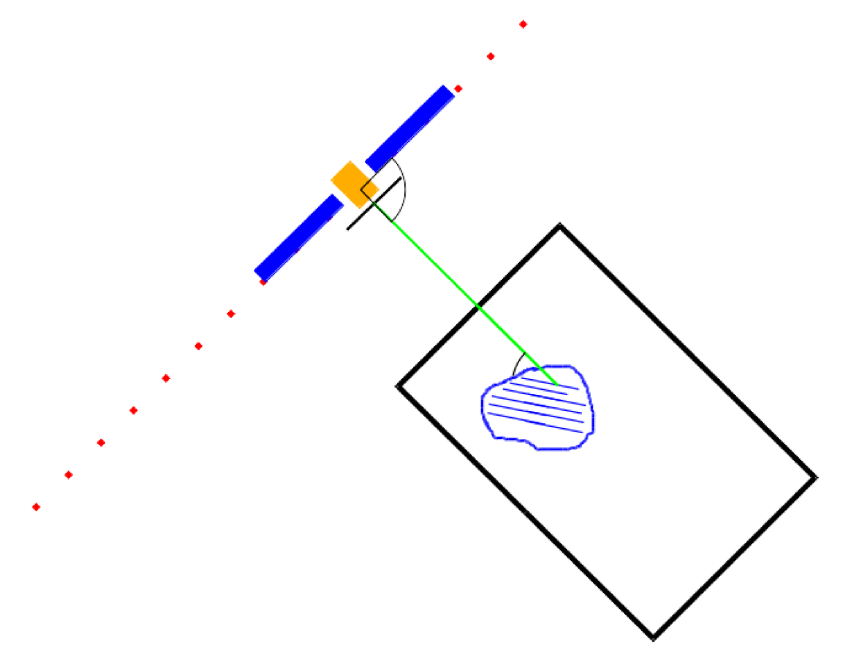

Figure 2.12: $\Phi$ is measured as degrees from incident electromagnetic wave to the surface of the ice island. $0^{\circ}$ is a parallel orientation while $90^{\circ}$ is a perpendicular orientation.

In order to expand the understanding of spatial variability, the patterns of the SAR returns of the constituent ice types and surface were modelled (Dierking et al., 1994). Interestingly, when viewed parallel to the ridges $\left(\Phi=0^{\circ}\right), \theta$ was less critical, causing the ridges to appear as subtle elongated objects against their surroundings, but of less backscatter variability.

De Abreu et al. (2011) used a multi-image and GIS approach to examine the C-band SAR backscatter variability of ice islands in four polarizations (Figure 2.13). Primarily FQ, ScanSAR Wide and Narrow, and single-polarization $\mathrm{HH}$ and $\mathrm{HV}$ C-band RADARSAT-2 images across many $\theta$ were used to recommend the best polarization(s) for ice island detection in ScanSAR wide swath mode imagery. The factors controlling SAR variability in each polarization that were assessed were: geometry and type of ice island, ocean cover, ambient temperature, and SAR beam mode. 
Using tracking beacons placed on three ice islands from Ward Hunt and Markham ice shelves, the outlines of these ice islands were delineated in the FQ imagery. Using these outlines and a sample of the pixels around the ice island, the contrast between the ice island and the surrounding ice pack or open water could be determined. This surrounding pack ice was subsequently called the generic background, meanwhile other manually specified ice targets were delineated and called specific backgrounds (FYI or MYI). Lastly, due to the paucity of open water in the study, the backscatter from an open water background was modelled for various wind speed conditions (De Abreu et al., 2011).

Detectability was measured based upon the measure

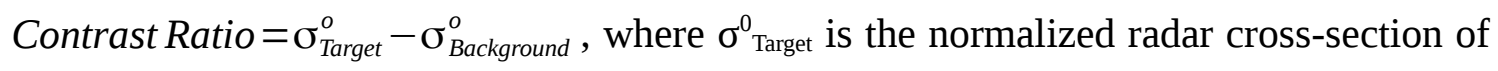
the target surface in decibels and $\sigma^{0}$ Background is the normalized radar cross-section of the background surface in decibels. Owing to the $\log$ transform in $\sigma^{0}$, the result is the ratio of the target to background backscatter in linear power scale.

An initial bivariate analysis was used to examine the relationship between the contrast ratio and date, temperature and $\theta$, amongst other variables. Analysis of Covariance (ANCOVA) was also used with repeated measures, with a random effect, fixed effect of two factors (polarization and ice island ice type) and two covariates ( $\theta$ and ambient temperature) in order to account for the pseudo-replication in the dataset. Each ice island target had numerous samples over the series of images, which violates one of the assumptions of ANCOVA. The pseudo-replication violation was corrected with a linear mixed effect model, effectively ANCOVA with repeated measures. ANCOVA was used to determine which polarization was the most useful to detect an ice island against 
specific, generic and open-water backgrounds, despite the confounding factors which can affect SAR returns. Generally speaking, the cross-polarized channels (HV and VH) were found to be most suitable for detecting ice islands (De Abreu et al., 2011).

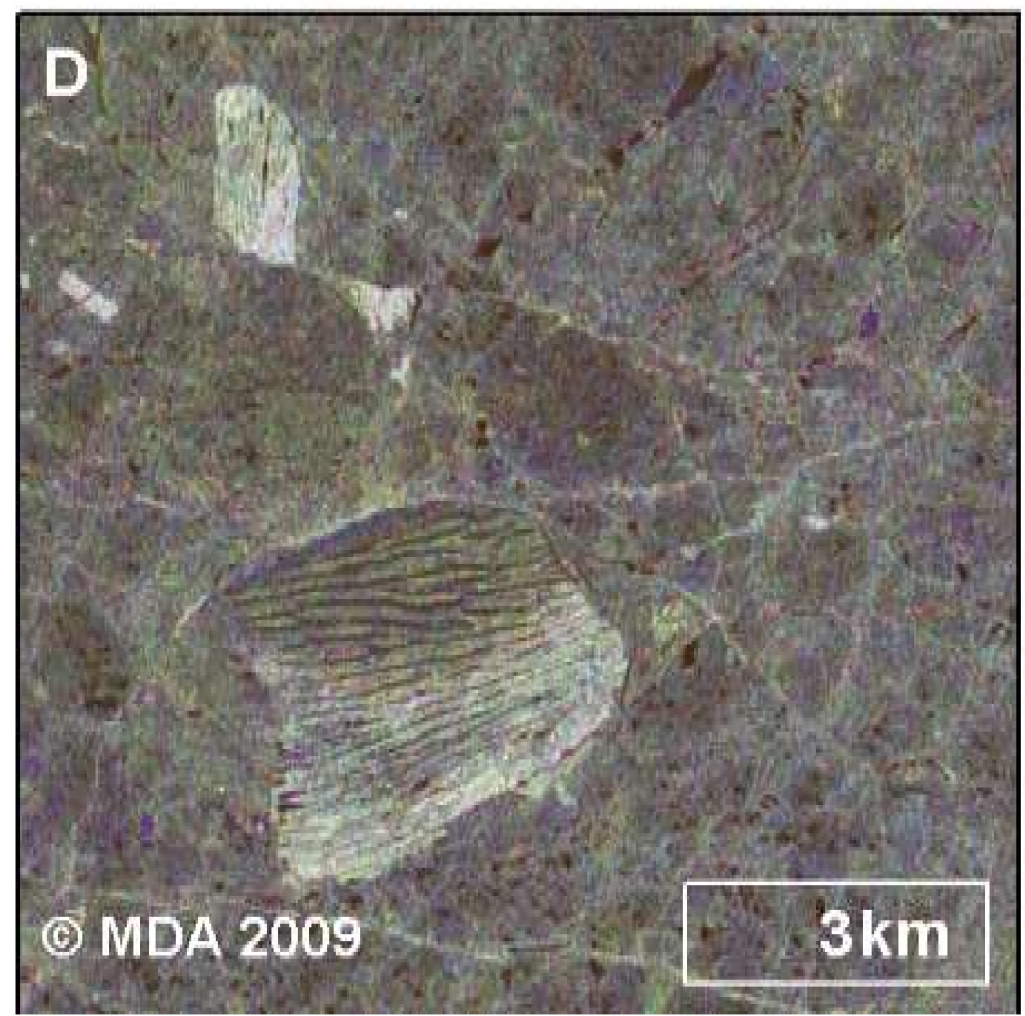

Figure 2.13: Ice island appearance in SAR as a Pauli decomposition, red $=H H+V V$, green $=2 * H V$, blue $=H H-V V$. This figure shows the characteristic surface ridges on an ice island during summer melt conditions. Pictured are Target 7 (Markham M1 ice island) in the middle, with a smaller ice island fragment to the upper left with marine ice. The surrounding sea ice is darker with less backscatter. FQ3, $6.3 \mathrm{~m}$ pixel size, 2009/07/13 15:27:33 GMT (De Abreu et al., 2011)

Some of the SAR variability can be accounted for by differing $\theta$ (De Abreu et al., 2011). The backscatter of any surface is influenced by $\theta$. The received power is defined by:

$$
P_{r}=\frac{\lambda^{2}}{(4 \pi)^{3}} \int_{A} \frac{P_{T} G^{2} \sigma^{o}}{R^{4}}
$$


where $P_{T}$ is the power transmitted, $\lambda$ is the wavelength, $G$ is the antenna gain, $R$ is the range to the surface, $\mathrm{A}$ is the illuminated area and $\sigma^{\circ}$ is the radar scattering coefficient. The radar-scattering coefficient itself is a combination of frequency, $\theta$ and polarization, dielectric constant, and roughness (Onstott, 1992; Onstott and Shuchman, 2004; Yackel and Barber, 2000). It is well understood that $\theta$ can influence ice type classification and diminish accuracy, especially between open-water and sea ice at steep $\theta$ in single polarization SAR imagery (De Abreu et al., 2011). Results by Gill and Yackel (2012) have shown quantitatively that polarimetric variables show varying degrees of sensitivity to $\theta$, depending upon the surveyed ocean cover (Gill and Yackel, 2012).

De Abreu et al. (2011) also explored how differing wind speeds over open-water influence ice island separability. Generally speaking, calm water produces mostly specular reflection. Increasing wind augments the roughness of the ocean, leading to higher backscatter due to Bragg scattering (Shokr and Sinha, 2015). From Figure 2.14, it is evident that regions such as on the leeward side of the islands where wind and waves are diminished, appear darker. Meanwhile, regions that would experience greater wave action have a greater SAR return. 


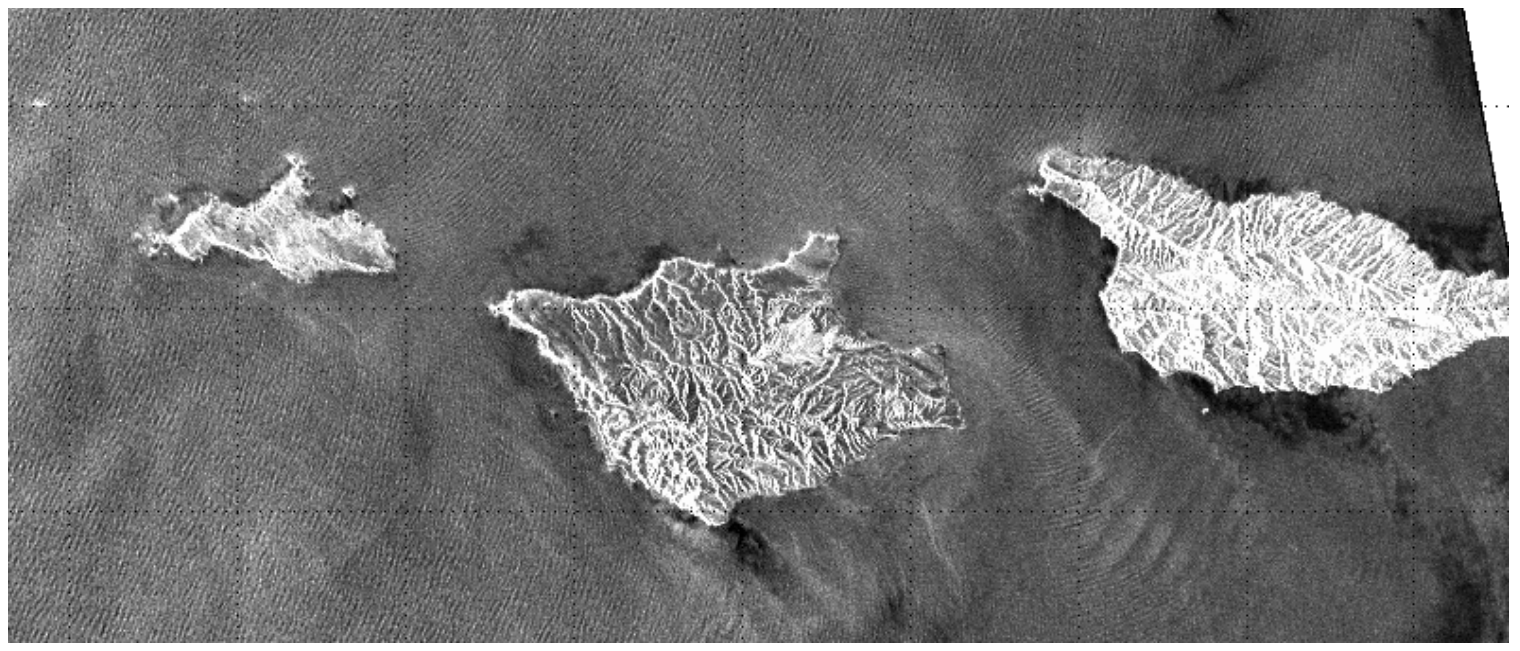

Figure 2.14: Changing SAR response due to wind and waves around islands. The pattern of high and low backscatter is consistent with regions that are exposed and sheltered from wind, respectively (BoostTechnologies, 2006).

\subsubsection{Sea Ice}

Scheuchl et al. produced multiple papers (2001a, 2001b, 2002, 2005) exploring the use of multiple polarization SAR images to discriminate between ice types. They recognized that the additional information afforded by polarimetry could assist in detection during challenging environmental conditions, such as the summer melt. SAR variables such as $\mathrm{H}, \mathrm{A}, \alpha$ were able to aid in classification with a SIR-C quadpolarimetric image of an $\theta$ of $26^{\circ}$ to $31^{\circ}$ (Scheuchl et al., 2001a, 2001b). Decompositions such as the Freeman-Durden decomposition were a further improvement over $\mathrm{H} / \mathrm{A} / \alpha$ classifications (Scheuchl et al., 2005, 2002) with an AirSAR C- and L-band quadpolarimetric image covering an $\theta$ of 22 to $52^{\circ}$. The usefulness of quad polarimetry for ice 
classification was confirmed by all of the investigations by Scheuchl et al. (Scheuchl et al., 2005, 2002, 2001a, 2001b).

Although several researchers have used polarimetry for sea ice classification, most have not used more than a few images with very few polarimetric SAR variables. This low sample size over a low number of viewing conditions limits the understanding of variability of sea ice classification. De Abreu et al. (2011) used 478 images, of which 64 were quad-polarimetric. However, only backscatter was assessed without using any polarimetric variables. Jeffries and Sackinger (1990) assessed a single SAR image and Dierking et al. (1994) used a small number of images. Furthermore, each of the Scheuchl et al. studies (2001a, 2001b, 2002, 2005), conducted training and classification within the same image, not considering other viewing or environmental conditions. This probably

resulted in inflated accuracy due to spatial autocorrelation between training and validation data. While there are many examples of assessments of SAR variability of ice islands such as those listed above, as well as examples of sea ice classification by Scheuchl et al. (2001a, 2001b, 2002, 2005), the combination of multi-image, multipolarimetric techniques has not been attempted before.

\subsection{Background to the Analytical and Mapping Techniques used in this Research}

In the development of this thesis, several statistical tools such as ANOVA, Tukey's Honest Significant Differences (THSD) and Games-Howell (G-H) were used to select the best SAR variables to discriminate each ocean cover. Redundancy Analysis (RDA) was used to assess gradients which explained the greatest variability in the selected SAR 
variables. Finally, Simple Linear Iterative Clustering (SLIC) was used to segment images into objects for classification with the trained model and Support Vector Machines (SVM) were selected to train a classification model using the selected SAR variables.

\subsubsection{Separability Measures to Assess SAR Variables}

In remote sensing, there are several ways to determine overall usefulness of a SAR variable in terms of separating classes. Transformed Divergence or Bhattacharyya Distance are other techniques (Richards, 2012). Transformed Divergence is a method of aggregating classes based on a desired accuracy (Richards, 2012). Bhattacharyya Distance is a measure of probability of classification between a pair of classes (Mather and Koch, 2011). It is possible to use statistical tests such as ANOVA or THSD/G-H to assess difference of means between classes.

\subsubsection{Determination of Overall Separability with ANOVA}

ANOVA is a method to compare differences of the means in three or more groups. This method assesses the degree of variation amongst groups with the degree of variation within the groups. The null hypothesis in ANOVA is that all the population means are equal. ANOVA makes the assumptions that response is normally distributed and that variance is similar between different groups (De Veaux et al., 2012).

\subsubsection{Determination of Specific Pairwise Separability with Tukey's Honest Significant Differences and Games-Howell tests}

Although ANOVA can provide a significant result suggesting that at least one population mean is different from the others, it cannot inform as to which of the three or more populations differ from each other. Post-hoc pairwise comparisons are necessary to 
explore this and several approaches can be used to identify significant pairwise differences such as THSD, Scheffe's method and G-H. Two of these will be explained further.

THSD can provide the real difference between two means using the Student's distribution (called the Q distribution). This leads to a sampling distribution of the largest difference between a set of means of the same distribution. All pairwise differences use

the identical Q distribution, leading to a conservative THSD assessment (Abdi and Williams, 2010). THSD is superior to the t-test in that the experiment-wide error rate is corrected for, reducing the chance of a Type I error (Schumacker, 2014). THSD has the same normality and homogeneity of variance assumptions as ANOVA. THSD is somewhat insensitive to the assumption of normality and as such, some non-normality is generally considered acceptable (McDonald, 2008).

The G-H test is similar to THSD in that it provides a statistical measure to difference of means test. However, the $\mathrm{G}-\mathrm{H}$ test does not have the same assumption of variances as THSD although it still assumes normality. The G-H test computes the degrees of freedom for each pairwise comparison (Wilcox, 2003) and, as such, takes into account the homogeneity of variance of groups (Games et al., 1979; Wilcox, 2003). The null hypothesis in both tests is the same as that in ANOVA: all the population means are equal.

\subsubsection{Direct Gradient Analysis}

The goal of direct gradient analysis, also known as ordination, is to explain one dataset by another related dataset (Legendre and Legendre, 1998). For the assessment of 
variability along detected direct gradients, RDA and Canonical Correspondence Analysis (CCA) are common approaches that are similar to each other. They are both also similar to Principal Component Analysis (PCA) but extend the ordination by modelling response variables with respect to explanatory variables (Zuur, 2007). In this way, these techniques are similar to multiple regression but can accommodate more than a single response variable. RDA and CCA can describe what percentage of the variance in the response variables is explained, and provide significance values for the explanatory gradient axes (Kindt, 2005) that describe the likelihood that the observed pattern is a random occurrence. Ordination may also be extended with partial ordination analysis, where the variance that can be explained by some subset of the response variables is removed, so that only the remaining variance may be explained by a certain subset of the response variables. The distinction between RDA and CCA is that RDA assumes an approximately linear response to the explanatory variables while CCA assumes a unimodal response (Šmilauer and Lepš, 2014; Zuur, 2007). Furthermore, RDA can detect gradients in variables whereas CCA cannot (Gardener, 2014). The suitability of RDA or CCA may be assessed by testing the total explained variance (Simpson, 2011). RDA and CCA are multivariate exploratory methods that can be used to visualize the relationships between many variables. Multivariate Regression Trees (MRT) are also used for this purpose but are more commonly applied to discrete explanatory variable types (De'ath, 2002).

The null hypothesis in RDA and CCA is that the response variables are unrelated to the explanatory variables. A Monte-Carlo approach to testing significance relies upon generating a large number of datasets. Then, the F-ratio test-statistic is recomputed for 
each new dataset and the significance level is estimated. From the range of F-ratios, the RDA or CCA significance of the original dataset may be computed (Šmilauer and Lepš, 2014).

Ordinations are typically displayed as biplots which show the relative importance of each variable and associations between variables and the two ordination axes (Legendre and Legendre, 1998). Variables are denoted by arrows that are anchored at the graphs origin. Samples or observations are plotted as discrete points. When samples are also graphed, the term triplot is also used. The cosine of the angle between any two variables or an RDA/CCA axis and a variable represent the approximate correlation (Legendre and Legendre, 1998). Angles of $90^{\circ}$ represent no correlation while angles of $0^{\circ}$ represent positive correlation. Angles of $180^{\circ}$ represent negative correlation (ter Braak, 1990). The relative length of an arrow represents the importance of the variable to the RDA/CCA model (Torontow and King, 2011). The distance between samples has no meaning when using correlation scaling as described above (ter Braak, 1990).

The best way to describe an RDA/CCA triplot is with an example. See Figure 2.15 for an example of an RDA ordination plot (Torontow and King, 2011). The goal here was to relate forest complexity variables from field measurements to remote sensing variables from a Quickbird scene. Red arrows are observed variables from Quickbird imagery (average angular second moment, average shadow, average correlation, standard homogeneity and Normalized Difference Vegetation Index (NDVI)) or DEM (average slope). Blue measurements are in-situ measurements of forest complexity. Arrows of increasing forest complexity were added to clarify meaning of measured field variables in 
relation to forest complexity.

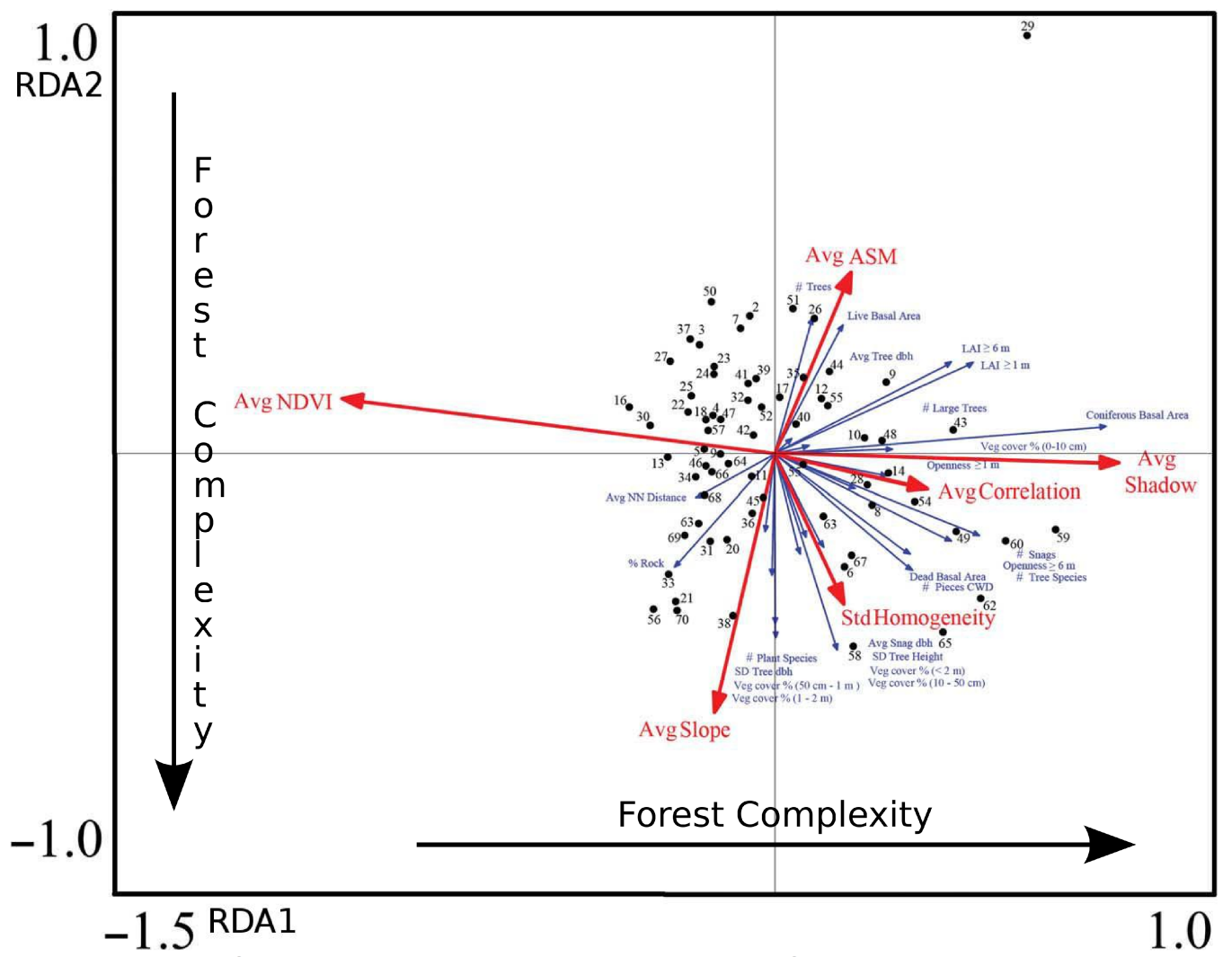

Figure 2.15: Triplot for RDA axes 1 and 2 ( $x$ and $y$ ) with highest forest complexity in lower right and lowest forest complexity in upper left. Red arrows are variables derived from Quickbird imagery or DEM and blue arrows are field measurements. Numbered points are individual field samples (Torontow and King, 2011).

From this triplot, one can see that the explanatory variables Avg Correlation and Avg Shadow are positively and strongly correlated (the angle between them is small) and both are highly negatively correlated to NDVI (angle $>180^{\circ}$ ) which is the most important variable in this model (longest arrow). Avg Shadow is closely related to the first RDA axis but is not associated with the second (perpendicular). This is also the case for the response Coniferous Basal Area, which can be explained by a high degree by RDA1 and Avg Shadow. Along the RDA1 axis, as forest complexity increases, canopy openness is 
greater but there is higher LAI representing multiple forest layers, coniferous basal area and the number of large trees is higher, and the number of dead trees (snags) is higher. This is manifested in imagery through increased image texture and shadow, and decreased NDVI (Torontow and King, 2011).

\subsubsection{Segmentation and Classification Techniques}

Segmentations are used to convert pixels into larger objects that are then identified with a classification method. Segmentation approaches are often used in classifications to combine many pixels into objects that are more representative of the target. For instance, per-pixel classifications with radar data may lead to erroneous results due to artifacts of image speckle (Devadas et al., 2012; Xiao-xia et al., 2006). Object-based classifications can include the geometry of nearby pixel values to enhance the classification (Liu et al., 2010). There are many segmentation approaches available, many of which are designed and tuned for narrow uses. One example is the SLIC approach which is used in medical imaging of micrographs (Achanta et al., 2010) where the boundaries are poorly defined and the images are quite noisy.

Once an object-based segmentation is performed, a classification technique uses the available pixel information and a series of training images to generate a classification model. This model is then used to classify new image. There are also many possible classification techniques, such as Random Forests, that incorporate non-linear classification rules (Chen, 2007). SVM also is a non-linear classifier which works well with large multivariate datasets. 


\subsubsection{SLIC Segmentation for Object-based Classification}

SLIC performs clustering based on pixel similarity and proximity across all bands in the image plane. Pixel similarity provides 3-dimensions (or better understood as image bands) in the CIELAB-space. The CIELAB-space is a colour-space (as is RGB) that uses Lightness, A and B (as opposite colours) for colour representation as opposed to Red, Green and Blue. CIELAB is considered uniform across small colour distances, which is needed for the SLIC implementation (Achanta et al., 2010). SLIC has not been expanded to use more than three input bands. For spatial pixel proximity, a normalized 2dimensional ( $\mathrm{x}$ and $\mathrm{y}$ ) distance is used which enforces approximate cluster sizes and spatial extent. The pixel proximity provides the possibility of varying the compactness of the cluster. Pixel similarity and proximity are combined in a 5-dimensional spatial-colour space (Achanta et al., 2010). From this 5-dimensional space, colour similarity and pixel proximity are enforced so that the cluster sizes and their spatial extent are approximately similar thereby simplifying image classification (Achanta et al., 2010). Distance-limited K-means aggregation is used to aggregate nearby 5-dimensional clusters based on both colour and spatial proximity (Achanta et al., 2012). SLIC offers many parameters to modify the segmentation. The number of segments parameter approximately controls the size of each segment. The compactness parameter defines how regular the segments are to be at the expense of adherence to boundaries (Achanta et al., 2010). Also, the number of k-means iterations, pre-smoothing gaussian kernels, and minimum and maximum cluster sizes may be modified as well (scikit-image developers, v0.11dev). Minimum and maximum cluster sizes are based on the proportion to the average cluster size. 


\subsubsection{SVM Classification}

SVM is an approach for non-linear data classification and regression (Yu and Kim, 2012). SVM has been historically used for handwriting recognition but also has uses in arbitrary data classification and regression (Bahlmann et al., 2002). Experience in recent investigations has shown SVM to be an effective tool in both ice and water separation (Leigh et al., 2014) as well as ice stage of development (Huiying et al., 2014) in SAR. Non-linear classification rules were capable of yielding high accuracy (Leigh et al., 2014).

SVM uses kernel methods, which enables the use of linear and non-linear decision boundaries. Any SVM classification attempts to optimize or maximize the margins on either side of the classification rule (also known as a splitting plane or hyperplane) to elements of either class (Yu and Kim, 2012). In Figure 2.16, solutions A-D are all acceptable classification rules as they completely separate the classes. However, solution $\mathrm{D}$ is optimal as the splitting plane is furthest from the class elements. 

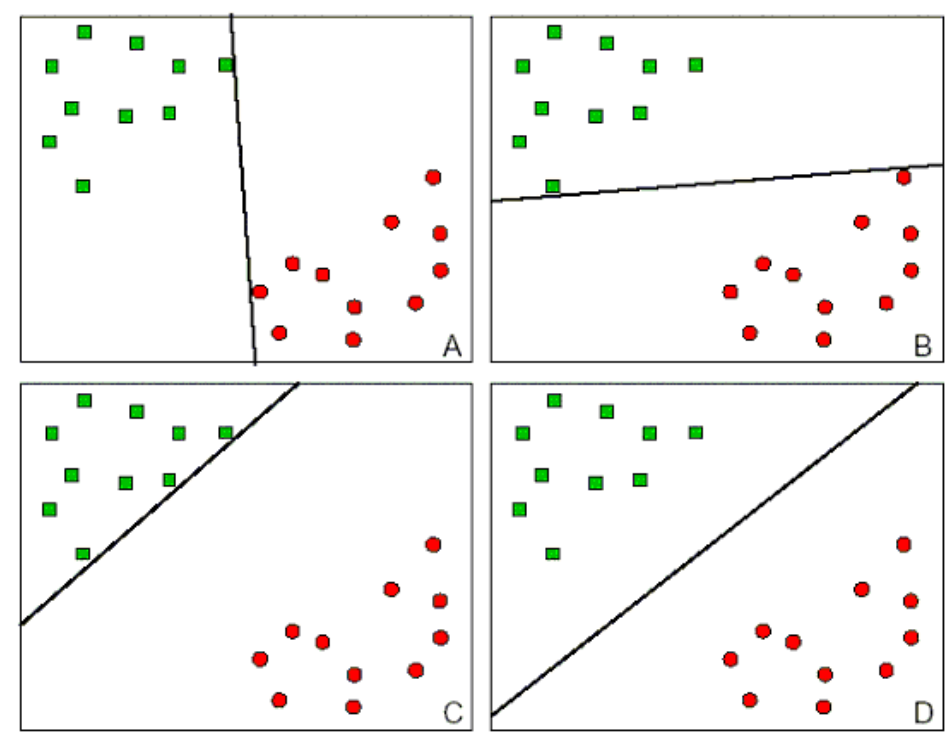

Figure 2.16: Example of maximum margin linear classification in SVM. $D$ is a superior classification to $A, B, C$ as the overall separation (distance of class members to the class boundary) is maximized to the splitting plane (Improved Outcomes Software, 2014).

However, some classifications, such as SAR classifications, are not linearly separable (Huiying et al., 2014; Leigh et al., 2014). In order to understand how other kernels can provide a non-linear classification, it would be useful to understand a one-dimensional space classification as in Figure 2.17 (Manning et al., 2008).

In Figure 2.17a, a one-dimensional dataset with a simple classifier with the hyperplane at 0 is shown. In Figure 2.17b, the dataset is no longer linearly classifiable with the additional data. However, by using some function (in this case, quadratic), additional dimensions may be added which allows for linear separation as in Figure 2.17c. When a nonlinear classification (Figure 2.18a) is projected into higher dimensional space as in Figure 2.18b, the complex classification may be reduced into a simpler linear classification in the higher dimensional-space. This concept also expands when there is 
higher dimensionality than demonstrated in Figure 2.18.

a)

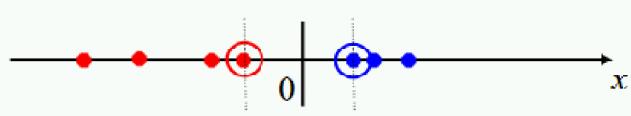

b)

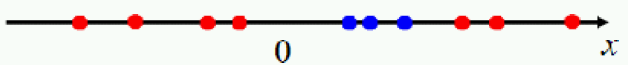

c)

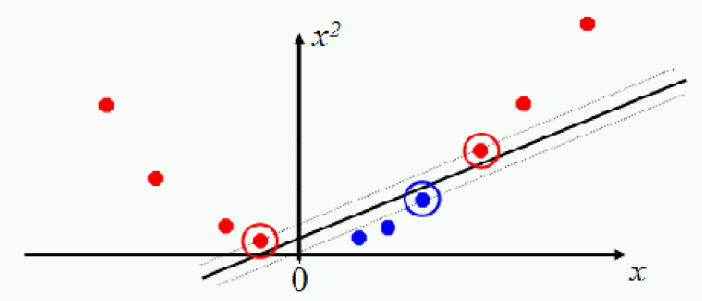

Figure 2.17: Computation of classifications in one-dimensional space. A simple linearly classifiable dataset with one dimension (a). A more complicated dataset which cannot be classified with a linear kernel (b). Projection of (b) into a higher dimension using some function to allow for a linear classification (c) (Manning et al., 2008)

a)

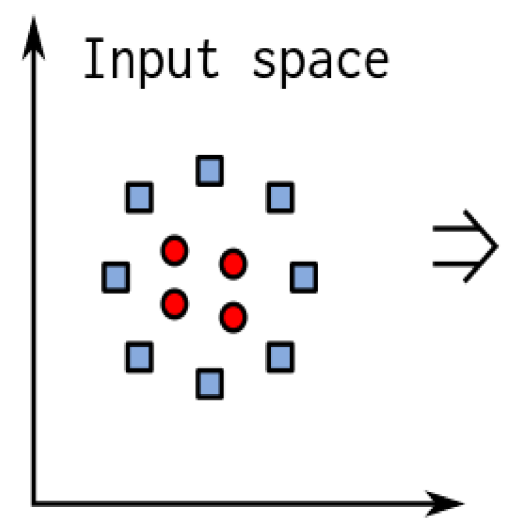

b)

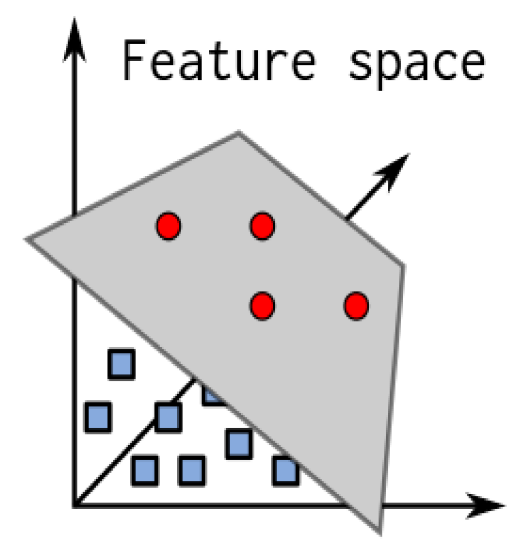

Figure 2.18: Computation of classifications in two-dimensional space. Non-linear classifications may be preferable to separate the two groups (a). These classifications, when raised to higher dimensions (b) allow a similar splitting hyperplane. Different classification hyperplanes may exist depending on the kernel used (Wagner, 2012). 
For these cases, a Radial Basis Function (RBF), Polynomial or Sigmoid kernel can provide an optimal hyperplane boundary (Figure 2.19). RBF is a non-linear kernel function that generally performs well in most situations. Furthermore, the RBF kernel has few parameters to optimize and has fewer numerical issues unlike the polynomial or sigmoid kernels (Hsu et al., 2010).
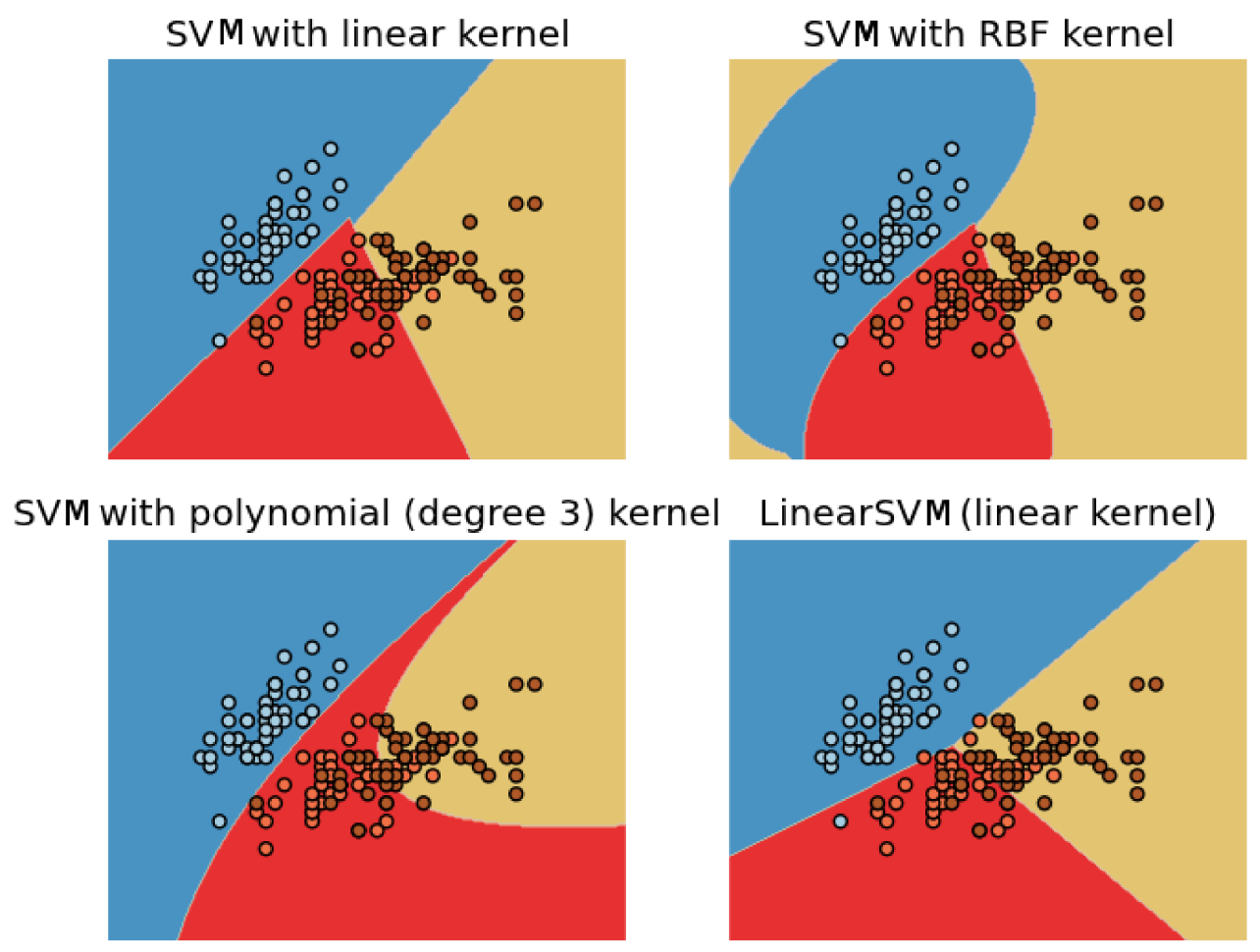

Figure 2.19: Different kernels for more complex classification splitting hyperplanes. These kernels are different functions which are used to elevate the dataset to higher dimensions (scikit-learn developers, n.d.).

The difference between these kernels, shown in Figure 2.19, is they are simply different functions applied to raise the dataset to higher dimensions, much like the quadratic function in Figure 2.17c.

With an SVM, the C parameter describes the slackness or how strictly the 
classification rule must separate the classes completely. Small values of C lead to a classification rule that has a larger margin separating hyperplane which may have misclassified points while a large value of $\mathrm{C}$ leads to a smaller-margin hyperplane which tries to classify more points correctly (scikit-learn developers, v0.16.0).

In addition to $\mathrm{C}$, there is the $\Upsilon$ parameter in RBF which influences the behaviour of the splitting hyperplane. $\Upsilon$ is the radius of influence of points selected to be support vectors of the hyperplane. Low values of $\Upsilon$ allow for a far ranging influence while high values of $\Upsilon$ mean a reduced range of influence (scikit-learn developers, v0.16.0). Optimization of these parameters may be performed with an iterative search where the classifier accuracy is estimated using cross-fold validation for every combination of $\Upsilon$ and $\mathrm{C}$. From this iterative search, the combination of $\gamma$ and $\mathrm{C}$ that maximizes the accuracy may be selected (Hsu et al., 2010).

It should be noted that SVM can have difficulty with unbalanced data where the classes do not have approximately equal populations. Classifier success is usually calculated with an equal importance assigned to misclassification of the majority class and misclassification of the minority class (Ben-Hur and Weston, 2010). Based on this principal, the successful classification of the majority class can outweigh the unsuccessful classification of the minority class. However, different weights can be assigned for misclassification depending upon class (Ben-Hur and Weston, 2010), which can lend more weight to the successful classification of the minority class. 


\section{METHODS}

\subsection{Radar Data and Site Selection}

For this study, 70 FQ and FQW images were used of various ice islands across the western and eastern Arctic (Appendix B). Most images were in the FQ operating mode while a few were also in the FQW mode (Table 3.1).

Table 3.1: RS2 operating mode coverage.

\begin{tabular}{|c|c|c|c|c|c|c|}
\hline \multirow{2}{*}{ Position } & \multirow{2}{*}{ Beam Mode } & \multirow{2}{*}{$\begin{array}{c}\text { Approximate } \\
\text { o Angle } \\
\text { Range for } \\
\text { Position }\end{array}$} & \multicolumn{2}{|c|}{$\begin{array}{l}\text { Nominal Range Resolution } \\
\text { (m) }\end{array}$} & \multicolumn{2}{|c|}{ Number of scenes } \\
\hline & & & FQ & FQW & $\mathbf{F Q}$ & FQW \\
\hline $\begin{array}{l}\text { Very Near } \\
\text { Range }\end{array}$ & $\begin{array}{c}\text { FQ1-6 / FQW } \\
1-7\end{array}$ & $18^{\circ}-25^{\circ}$ & $16.5-11.7$ & $17.3-11.0$ & 15 & 1 \\
\hline Near Range & $\begin{array}{l}\text { FQ7-12/ } \\
\text { FQW 8-12 }\end{array}$ & $25^{\circ}-31^{\circ}$ & $12-9.5$ & 11.8-9.4 & 12 & 6 \\
\hline Mid Range & $\begin{array}{l}\text { FQ13-18 / } \\
\text { FQW 13-18 }\end{array}$ & $31^{\circ}-37^{\circ}$ & $9.7-8.3$ & $9.9-8.2$ & 16 & 4 \\
\hline Far Range & $\begin{array}{l}\text { FQ19-24 / } \\
\text { FQW 19-21 }\end{array}$ & $38^{\circ}-42^{\circ}$ & $8.4-7.5$ & $8.5-7.8$ & 10 & 3 \\
\hline $\begin{array}{l}\text { Very Far } \\
\text { Range }\end{array}$ & FQ25-31 & $42^{\circ}-48^{\circ}$ & $7.5-6.8$ & & 3 & 0 \\
\hline & & Total & & & 56 & 14 \\
\hline
\end{tabular}

The ice islands under study originated from the Markham and Ward Hunt ice shelves, as well as from the floating ice tongue of the Petermann Glacier and drifted throughout the Arctic (Figure 3.1). Figure 3.2 and 3.3 show the parent ice shelf/glacier and the original location of each ice island in this study. These images were also used as a reference to determine the orientation of surface ridges to the major and minor axes of the ice island when such characteristics were not evident in SAR imagery. 


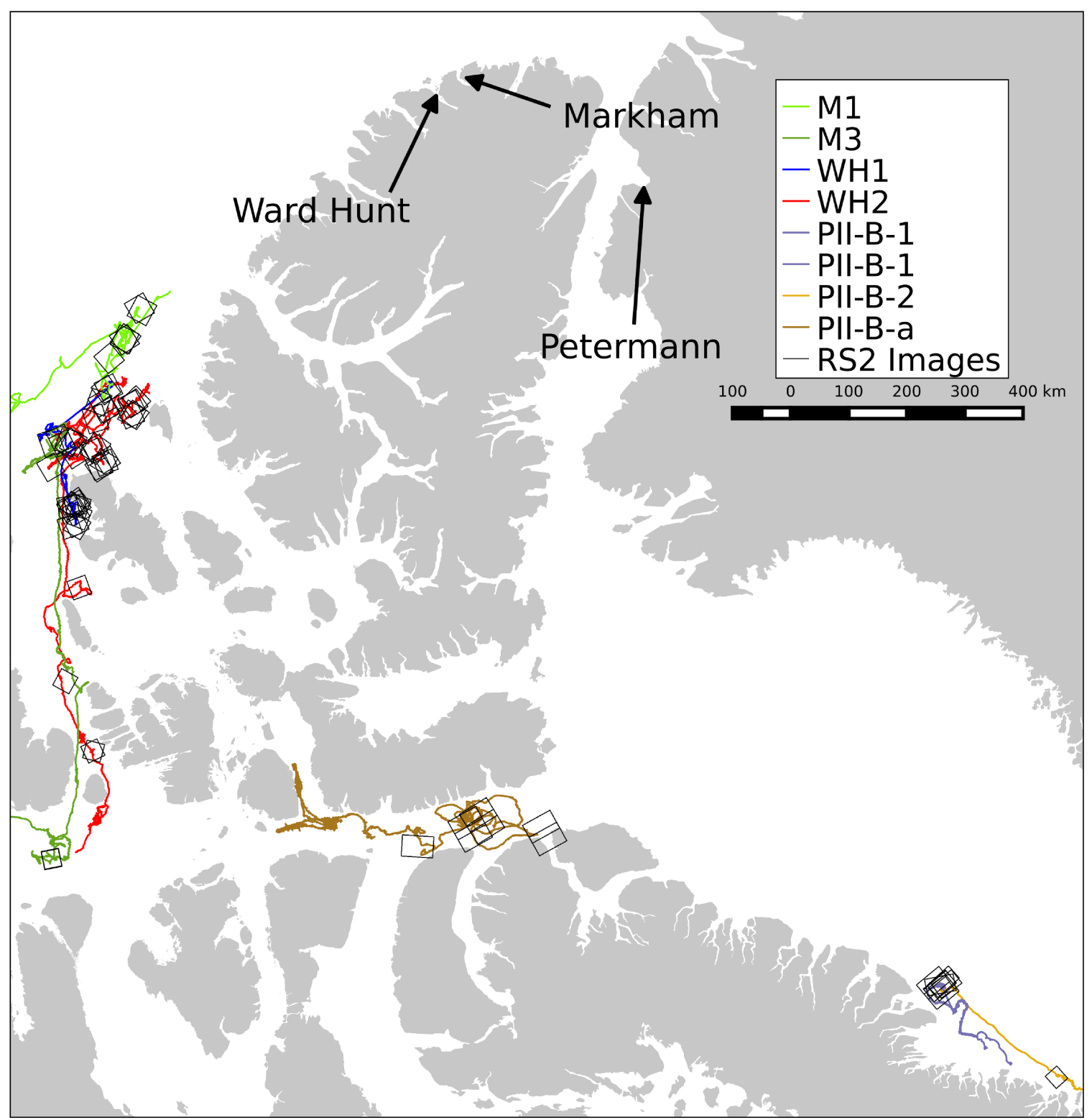

Figure 3.1: Ice island drift tracks, derived from tracking beacons and coverage of RS2 images listed in Table 3.2. The Petermann, Ward Hunt and Markham ice shelves and glacial tongues are also shown. 


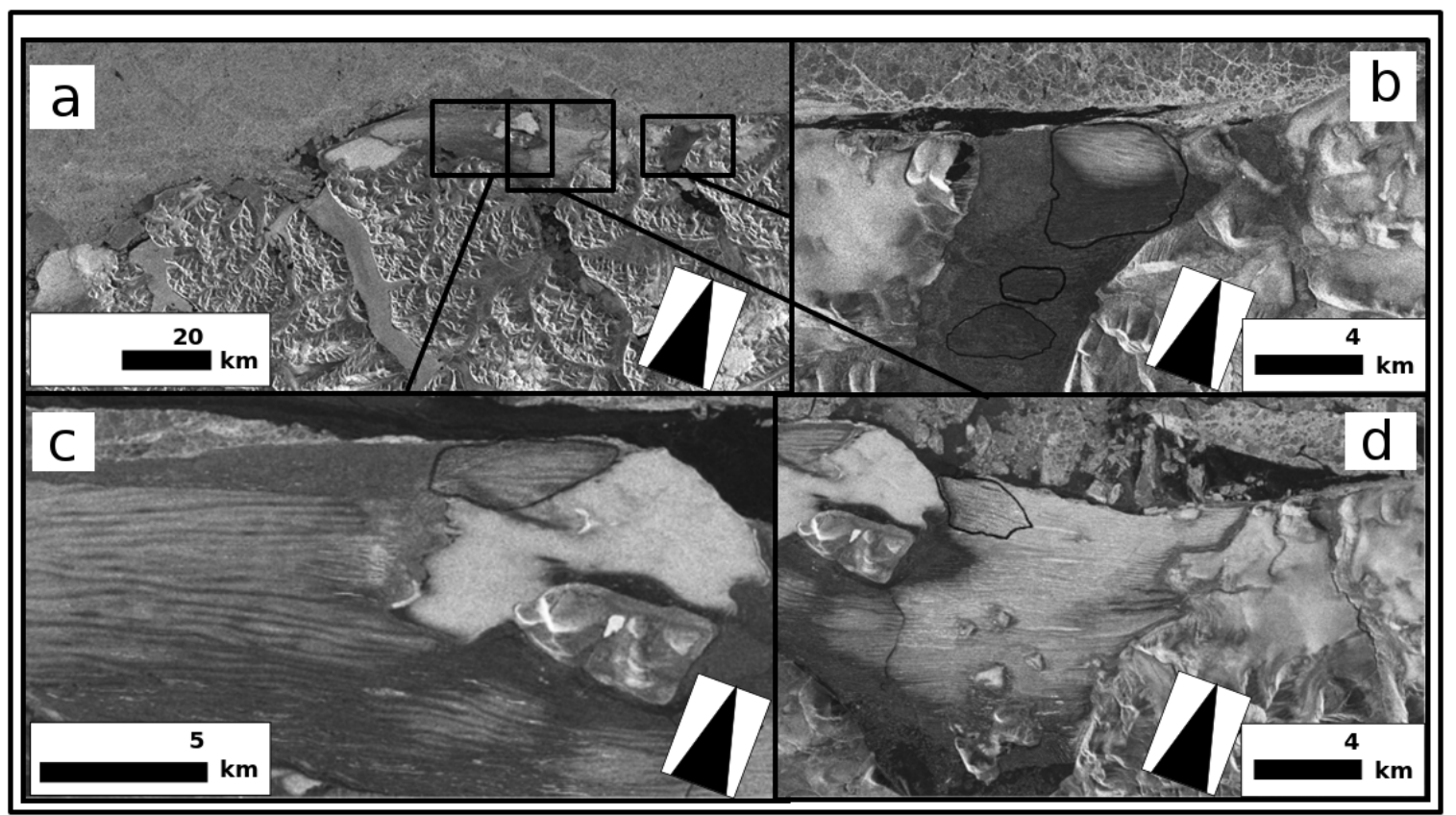

Figure 3.2: Ice island placement within the parent ice shelves at the northern coast of Ellesmere Island. a) Overview b) Markham Ice Island 1 (M1) in the upper right with Markham Ice Island 3 (M3) in the lower left (2007/01/21), c) Ward Hunt 1 (WH1) (2007/01/24), d) Ward Hunt 2 (WH2) (2008/02/02). All images are RS1. These images were used to orient the surface ridges relative to the overall ice island. Figure adapted from Crawford, 2013 


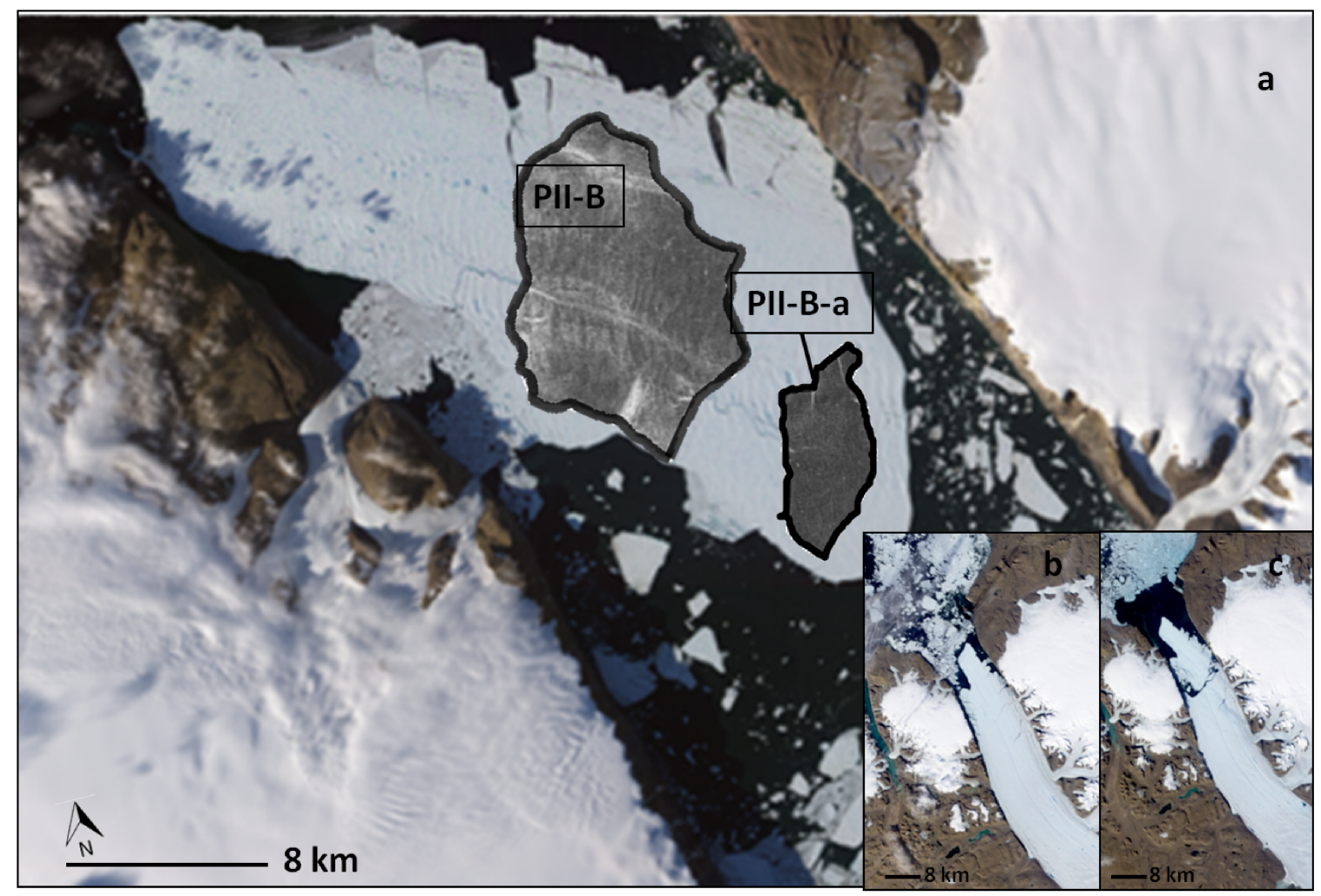

Figure 3.3: Petermann Glacier calving event in 2010 a) The placement of Petermann ice island fragments within the parent ice island and prior to further fragmentation (2010/08/16). b) The glacier terminus precalving (2010/07/21). c) The terminus and ice island post-calving (2010/08/05). Images are from the NASA MODIS Earth Observatory (NASA, 2010). These images were used to orient the surface ridges relative to the overall ice island. Figure adapted from Crawford, 2013

\subsection{Ice Island Tracking and Delineation}

In May and July 2009, following the calving events in 2008, three beacons were deployed on WH1, WH2 and M1 as part of the R2MS2 project (De Abreu et al., 2011). The Global Positioning System (GPS) beacon tracks were used to order FQ imagery and situate the ice islands within the imagery. From this location knowledge, the ice island outlines were digitized by Derek Mueller (De Abreu et al., 2011).

The other ice islands that were incorporated into this study were from Markham Ice 
Shelf (M3) and the Petermann Glacier tongue (PII-B, PII-B-1, PII-B-A). Beacons were placed on M3 in March 2010 by members of a Natural Resources Canada (NRCan) team gathering data for the United Nations Convention on the Law of the Sea and PII-B and PII-B-1 by Anna Crawford in 2011 as part of Project ICEBERGS (Crawford, 2013). The PII-B-A beacon was deployed in July 2011 by Julie Payette and Louis Fortier and revisited in October 2011 by Anna Crawford as part of Project ICEBERGS (Crawford, 2013). Image acquisitions were planned based on the location of the tracking beacons and ice islands with meteoric and marine ice were delineated by Anna Crawford and Derek Mueller. The outlines of each ice island included separate polygons for meteoric and marine ice (c.f. Mueller et al., 2006), based upon their appearance in Pauli decompositions and ancillary knowledge of the ice types from direct field observations of the parent ice shelves and optical imagery from FORMOSAT-2 and Moderate Resolution Imaging Spectroradiometer (MODIS). The ice island sample counts with the ice types measured for each ice island are in Table 3.2.

\begin{tabular}{|c|c|c|c|c|}
\hline \multirow{2}{*}{ Ice Island } & \multirow{2}{*}{ GRIP Report Identity } & \multicolumn{2}{|c|}{ Ice types } & \multirow{2}{*}{$\begin{array}{c}\text { Number of } \\
\text { FQ(W) Images }\end{array}$} \\
\hline & & Marine & Meteoric & \\
\hline PII-B-1 & N/A & $\checkmark$ & $\checkmark$ & 2 \\
\hline WH1 & Target 1 & $\checkmark$ & $\checkmark$ & 13 \\
\hline WH2 & Target 2 & $\checkmark$ & $\checkmark$ & 24 \\
\hline PII-B-A & N/A & & $\checkmark$ & 7 \\
\hline M1 & Target 7 & $\checkmark$ & $\checkmark$ & 10 \\
\hline M3 & N/A & $\checkmark$ & & 5 \\
\hline PII-B & N/A & & $\checkmark$ & 8 \\
\hline
\end{tabular}




\subsection{Ancillary Data}

In order to put the ice island geospatial data (described above) and the SAR data (described below) into context, ancillary data concerning the background ocean cover, air temperature, wind speed, $\theta$, and $\Phi$ was derived from various sources. Appendix B contains a summary of the measured environmental and SAR geometry variables of temperature, wind speed, $\theta$ and $\Phi$ per SAR image. Table 3.3 contains a summary of all the measured independent variables.

Table 3.3: Summary of all explanatory SAR geometry and environmental variables.

\begin{tabular}{|c|c|c|c|c|c|c|c|}
\hline Variable & Category & Symbol & Unit & $\begin{array}{c}\text { Level of } \\
\text { Measurement }\end{array}$ & Range & Source & Comment \\
\hline $\begin{array}{l}\text { Incidence } \\
\text { angle }\end{array}$ & Geometry & $\theta$ & degrees & Ratio & $19-50$ & $\begin{array}{l}\text { SAR image } \\
\text { metadata }\end{array}$ & $\begin{array}{l}\text { Angle of RS2 } \\
\text { beam to the } \\
\text { surface from } \\
\text { zenith }\end{array}$ \\
\hline Azimuth angle & Geometry & $\Phi$ & degrees & Ratio & $0-90$ & $\begin{array}{c}\text { Manual } \\
\text { classification }\end{array}$ & $\begin{array}{l}\text { Angle of RS2 to } \\
\text { the surface ridges } \\
\text { where } 0^{\circ} \text { is } \\
\text { parallel and } 90^{\circ} \\
\text { is perpendicular. } \\
\text { Not used for non- } \\
\text { ice islands }\end{array}$ \\
\hline Wind speed & Environment & & $\mathrm{m} \mathrm{s}^{-1}$ & Ratio & $0-17$ & GEM-GDPS & $\begin{array}{l}\text { Wind speed in } \\
\text { the SAR image }\end{array}$ \\
\hline Temperature & Environment & & $\mathrm{K}$ & Ratio & $227-278$ & GEM-GDPS & $\begin{array}{c}\text { Air temperature } \\
\text { in the SAR } \\
\text { image }\end{array}$ \\
\hline Feature & $\begin{array}{l}\text { Ice island } \\
\text { identity }\end{array}$ & & & Nominal & $\begin{array}{c}\text { M1, M3, } \\
\text { PII-B, PII- } \\
\text { B-1, PII- } \\
\text { B-A, } \\
\text { WH1, } \\
\text { WH2 }\end{array}$ & Beacon track & $\begin{array}{l}\text { Name of the ice } \\
\text { island. Not } \\
\text { included for non- } \\
\text { ice islands }\end{array}$ \\
\hline Shape & $\begin{array}{l}\text { Ocean cover } \\
\text { identity }\end{array}$ & & & Nominal & $\begin{array}{c}\text { water, } \\
\text { FYI, } \\
\text { marine, } \\
\text { meteoric, } \\
\text { MYI }\end{array}$ & $\begin{array}{l}\text { CIS Regional } \\
\text { Charts and } \\
\text { manual } \\
\text { classification }\end{array}$ & Ocean cover \\
\hline Offset & & & days & Ratio & $0-6$ & $\begin{array}{l}\text { CIS Regional } \\
\text { Charts and } \\
\text { SAR images }\end{array}$ & $\begin{array}{l}\text { The time delay } \\
\text { between the CIS } \\
\text { regional chart for } \\
\text { reference and the } \\
\text { SAR image. } \\
\text { Value of } 0 \text { for ice } \\
\text { islands. }\end{array}$ \\
\hline
\end{tabular}




\subsubsection{Identity Variable - CIS Ice Type}

CIS regional ice charts were used to provide a qualitative assessment of the surrounding ocean cover and ice types. Weekly regional ice charts were selected such that the time offset with SAR imagery was minimized by selecting the closest CIS chart in time to the RADARSAT-2 acquisition date, including when the SAR image overlapped with multiple regional charts. For each SAR image/ice chart pair, the chart area that was not covered by the extent of the SAR image was removed in addition to the area occupied by the ice island. The remaining area, which represents the background ocean cover, was reclassified as MYI, FYI or water based upon the egg codes in the ice chart (MANICE, 2005). This reclassification was to simplify the numerous subtype classifications provided by the regional ice charts into more aggregate classes that provide a better observation of SAR variables across all images. This reclassification simplified the numerous subtype classifications provided by the regional ice charts into more aggregate classes that provide a better observation of SAR variables across all images. The ice was reclassified based on the most common ice type in the polygon. If a dominant ice type was not apparent (4/10ths to 6/10ths), the polygon was not included in further analysis. Regions with less than 2/10ths ice cover were classified as water. Majority ice regions with stages of development coded as " 1 " - "9", "1." and "4." (which corresponds to ice

less than a year old) were classified as FYI. Majority ice regions with stages of development "7." - “9.” were classified as MYI (MANICE, 2005). All other ice classes were not included in the study. Table 3.4 contains the numbers of samples for each ocean 
cover.

Table 3.4: Ocean cover sample sizes. Note that marine and meteoric ice are ice island sub-types.

\begin{tabular}{cc}
\hline Ocean Cover & Number of Samples \\
\hline Marine ice & 53 \\
Meteoric ice & 65 \\
MYI & 51 \\
FYI & 15 \\
Water & 14 \\
\hline
\end{tabular}

\subsubsection{CIS Chart Offset}

Sea ice drift rates can vary considerably from no movement per day when ice is landfast to rates on the order of $20 \mathrm{~km}$ per day, which are possible for icebergs in open ocean around the Grand Banks of Newfoundland (Fisheries and Oceans Canada, 2010). Due to this possible rapid sea ice movement, the time offset between the CIS chart and image could lead to significant confusion in the identity of the ocean cover. The temporal mismatch between the CIS ice charts, which are based on ScanSAR imagery, and the FQ and FQW imagery used in this study was found to be 2 days or less for $58 \%$ and 3 days or less for $88 \%$ of the images (Table 3.5).

\begin{tabular}{|c|c|}
\hline $\begin{array}{l}\text { Maximum Offset } \\
\text { (Days) }\end{array}$ & Frequency \\
\hline 0 & 6 \\
\hline 1 & 15 \\
\hline 2 & 20 \\
\hline 3 & 21 \\
\hline 4 & 5 \\
\hline 5 & 2 \\
\hline 6 & 1 \\
\hline
\end{tabular}


The implications of this temporal mismatch were assessed qualitatively by aligning about one quarter of the SAR images and the corresponding CIS charts. Each ice region polygon was evaluated for how well it qualitatively corresponded to the underlying image. By this assessment, minor ice boundary deviation had minimal influence. The temporal offset between ice chart and SAR image was retained as an ancillary variable to test the effect of this factor in RDA.

\subsubsection{Environmental Variables - Wind / temperature}

Environmental variables were extracted from the output of the Global Environmental Multiscale model-Global Deterministic Prediction System (GEM-GDPS) which was the nearest temporally to the SAR acquisition date and time. The GEM-GDPS is a $0.24^{\circ} \mathrm{x}$ $0.24^{\circ}$ (approximately $25 \mathrm{~km}$ resolution), twice-daily dataset provided by the Canadian Meteorological Centre (Government of Canada, 2015). The zero hour nowcast at level 10000 was used (which corresponds to $1000 \mathrm{hPa}$, approximately at the ocean surface) in order to extract the most accurate forecast values possible for wind speed and temperature. The coordinates of the ice island feature centroid in each SAR acquisition were used to extract the surface air temperature and wind vectors from the appropriate GEM GDPS grid cell. The magnitudes of the $u$ and $v$ wind vectors were computed to provide wind speed $\left(\mathrm{m} \mathrm{s}^{-1}\right)$, and surface air temperature was given in Kelvin.

\subsubsection{Geometry Variable - Azimuth Angle to Surface Ridges}

$\Phi$ was computed by comparing the surficial ridging in Pauli decompositions of each 
ice island in SAR imagery to the path of incident microwave energy (Figure 3.4). In order to determine the direction of the incident microwave beam, Two-Line Element (TLE) files describing the orbital path of RADARSAT-2 were acquired from Celestrak (Kelso, 2015). Using these TLEs along with the Ground-Track-Generator (DeVona, 2013), the position of RADARSAT-2 at acquisition could be determined. The position was used to calculate the look direction of RADARSAT-2 which was used as reference for measuring the rotation angle of the ice island. For those images where the surficial ridging was not apparent in the imagery, the direction of these ridges was estimated from the reference images (Figures 3.2 and 3.3) and the major and minor axes of the ice island. This technique assumed that the ice island's shape was essentially unchanged since the reference image was acquired.

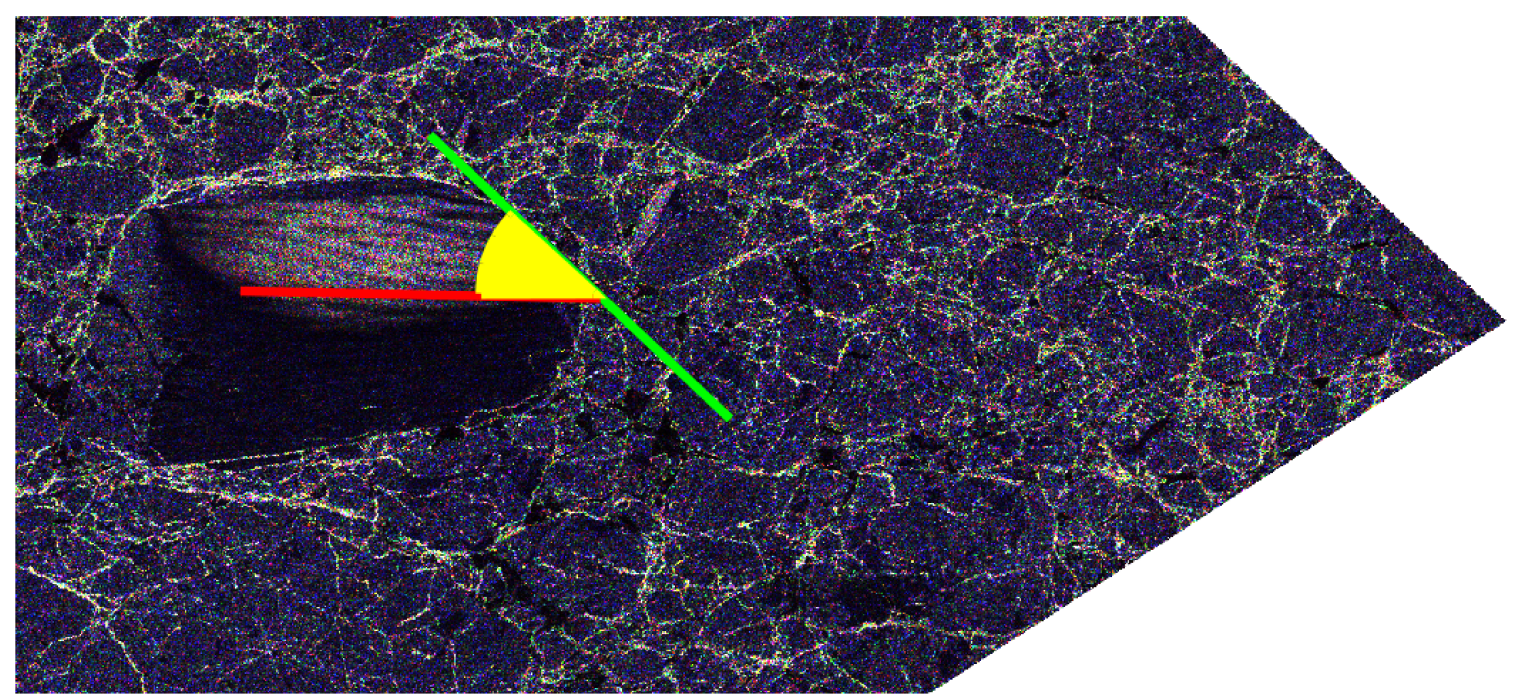

Figure 3.4: Example of measuring $\Phi$ for ice island M1. The green line indicates direction of incident $S A R$ beam. The red line is the prevailing ridge direction. The yellow arc is the measured angle. The RS2 image was acquired 2009/11/09. 


\subsubsection{Geometry Variable - Incidence Angle}

$\theta$ was computed from the image metadata, by averaging the near-range and far-range incidence angle. Although the incidence angle increases in the range direction across each SAR scene, an FQ or FQW scene is only 25 or $50 \mathrm{~km}$ wide, respectively, which equates to a $1^{\circ}$ to $4^{\circ}$ difference in $\theta$ from the near range to the far range (MacDonald, Dettwiler and Associates, 2013).

\subsection{SAR Variables}

Many of the SAR variables were chosen based upon prior literature (Scheuchl et al., 2005, 2002, 2001a, 2001b) or similarities to these SAR variables as well as possible candidates not explored in recent research that were promising for separating the recorded ocean covers. A wide diversity of SAR variables were examined since their usefulness could be determined using a statistical approach thereby allowing for an inclusive examination of the available data. Real and imaginary components of matrix elements were chosen from coherency, covariance and Sinclair matrices. $\alpha, \beta$, entropy and anisotropy were chosen based upon the ice classification literature (Scheuchl et al., 2005, 2002, 2001a, 2001b). Decompositions, such as Krogager, Neumann, FreemanDurden, Van Zyl and Touzi, were also included as they attempt to highlight a single scattering characteristic of the surface feature and could provide a simple understanding of the processes involved or a better distinction between the ocean covers. All the 123 SAR variables that were explored in this study are listed in Appendix B and use the names given in the PolSARpro software package (European Space Agency, 2014a). The summary statistics for the basic backscattering polarizations were computed. For clarity, 
$10 \mathrm{x} \log _{10}(\mid$ intensity $\mid)$ was used from the intensity matrix elements in PolSARpro (European Space Agency, 2014a) to convert to decibels. To compute the statistics, all values were converted to decibels before using the standard methods of calculating mean, median and standard deviation.

\subsection{Analysis}

The flowchart indicates the steps of the workflow that produced a dataset which could be explored statistically (Figure 3.5). A description of this flowchart with further details follows. 


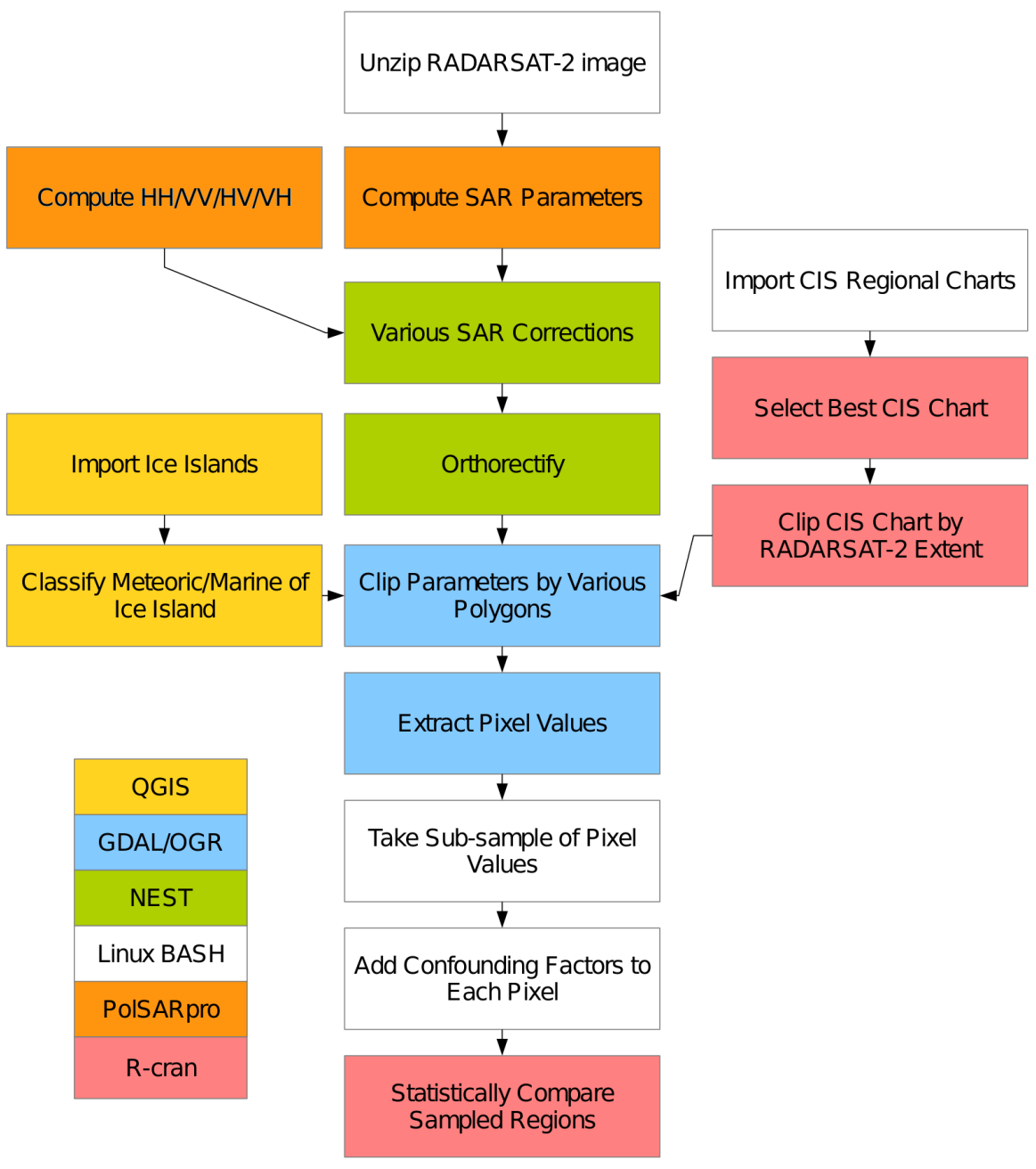

Figure 3.5: Flowchart of analysis methods. The colours indicate the software used.

\subsubsection{Computation of SAR Variables}

Using PolSARpro (European Space Agency, 2014a), the various SAR variables were computed for each image in slant range. In order to counter the variability of speckle, a multilook count of 3 and Lee Speckle filter of 5 pixels x 5 pixels were used based upon manual tuning of the multilook count and filter parameters. These chosen parameters 
match with other findings for Lee Speckle filtering (Mansourpour et al., 2006). This level of multilook and speckle filtering balanced low speckle and spatial resolution. Combining the multilook and Lee speckle filtering in $\mathrm{FQ}(\mathrm{W})$ polarimetric imagery led to approximately one-third the resolution, however, radiometric resolution was increased at the expense of spatial resolution. Since the ocean covers of interest are large in area, this trade-off was deemed desirable.

\subsubsection{SAR Correction and Orthorectification}

Next ESA SAR Toolbox (NEST) (European Space Agency, 2014b) was used to perform georeferencing after the computation of the SAR variables (European Space Agency, 2013). Radiometric normalization in the terrain correction tool was not used as the sigma-nought was previously computed in PolSARpro (European Space Agency, 2014a) and, furthermore, the images cover the ocean where the terrain is flat and radiometric normalization is not needed (Small et al., 2009). Terrain correction was applied using orbit state vector information from the image product as well as the Global Earth Topography and Sea Surface Elevation at 30 arc second resolution DEM (Bouvet, 2010). The terrain correction only applied to the area of the RADARSAT-2 scenes which contained a segment of land. The terrain correction was not strictly necessary but was automatically performed with the reprojection tools in NEST (European Space Agency, 2014b). The SAR variables were converted to ground range but remained unprojected in the native WGS84 coordinate system. The ocean cover shapefiles were originally projected as Albers Equal-area, latitude of first parallel $82^{\circ}$, latitude of second parallel $83^{\circ}$, latitude of false origin $82^{\circ}$, longitude of false origin $-75^{\circ}$, easting of false origin 0 , 
northing of false origin 0 . However, all clipping between the ocean cover shapefiles and the raster was performed in WGS84 with the shapefiles converted to WGS84 on-the-fly. This same Albers Equal-area projection was also used on-the-fly for determining the $\Phi$ angle and for all visualization.

\subsubsection{Further SAR Pre-processing}

Using these georeferenced SAR variables, as well as the manually classified ice islands and ocean cover regions from CIS ice charts, the SAR variables were clipped by the ocean cover regions. The pixel values were extracted and sub-sampled from these clipped regions. A 5\% random sub-sampling of the pixels was performed for the difference of means and RDA assessments to reduce the computational complexity and then the median was taken to represent each feature for further analysis. The median was chosen over the mean value as this measure of central tendency is robust to outliers and it enabled the use of lower spatial resolution training regions. In the case of SVM, the dataset was reduced to a $0.5 \%$ sub-sampling. A data set was constructed of all pixels that included the environmental and SAR geometry variables shown in Table 3.3. Date and timestamp were included to identify unique SAR images. With the added variables, the data was in a format that could be further explored statistically.

\subsubsection{Automation}

In order to prepare imagery, extract and organize data, a scripted toolbox was developed which enabled a consistent and systematic assessment of surfaces within the polarimetric images. The toolbox was designed to run automatically following the workflow of Figure 3.5 with only a few manual steps (the measurement of $\Phi$ and 
interpretation of the CIS egg codes as well as classifying the identity of ice islands). The software used includes QGIS v2.6.1 (QGIS Development Team, 2014), NEST v5.1 (European Space Agency, 2014b), PolSARpro v4.2 (European Space Agency, 2014a), and GDAL v1.10.0 (GDAL Development Team, 2014). All processing was performed on a Ubuntu 12.04 computer at the Water and Ice Research Laboratory at Carleton University using Python, Bash and R programming languages.

\subsubsection{Statistical Analysis}

To assess the best variables to improve the separability of ocean covers the median values of sampled ice island and ocean cover features were used as it enabled the use of lower spatial resolution reference data from the CIS regional charts and was less sensitive to time mismatch between the CIS chart and the SAR image than a pixel-based approach. A single median value was measured for each ocean cover feature in each SAR training image. The resulting distribution of median values was used in difference of means tests as well as the direct gradient analysis. These median summary statistics were used in all statistical analysis.

\subsubsection{Difference of Means Assessment}

The goal of the difference of means assessment was to reduce the number of SAR variables by determining which ones would be the most useful for classifying ice covers. First the assumptions of ANOVA, THSD and G-H were tested. The assumptions of normality and homogeneity of variance were tested with the Jarque-Bera and Levene tests respectively. The Jarque-Bera test was selected over the typical Shapiro-Wilk test for 
the assumption of normality as it assesses kurtosis as well as skewness (Koizumi et al., 2009). The second assumption, homogeneity of variance between groups, is especially important when the group sizes are unbalanced (McDonald, 2008). The Levene test was used to test homogeneity of variance as it is less sensitive to deviations from normality than the more common Bartlett test (Natrella, 2012). Due to the violation of the heteroscedasticity assumption, the G-H test was used in conjunction with THSD to ensure that the difference of means observed was significant taking into account differences of variance (Games et al., 1979; Wilcox, 2003).

An initial assessment with ANOVA was used to ascertain which SAR variables were and were not useful to separate ocean covers based on whether there was a significant difference of means. For the post-hoc differences of means tests, the G-H implementation used was an external library in R (Aoki, 2009) while the THSD implementation was an internal R function. SAR variables were deemed worthy of further investigation based on how separable the ocean cover was from other ocean covers using the THSD and G-H tests on the 70 SAR training images. The difference of means was comparing the distribution of the ocean cover median values across all the SAR images of the particular ocean cover in a specific SAR variable to the spread of median values in the same SAR variable for another ocean cover. The difference of means was normalized to the range of the SAR variable in question. For each of the five ocean covers, one SAR variable was selected which had the highest minimum normalized difference of means, which yielded SAR variables which had the greatest minimum separability compared to all other SAR variables for that target ocean cover. This separability measure has been termed the 
Maximum Base-Level Difference of Means Score. The separability score is unitless and ranges from 0 for no separability to 1 for complete separability from the next most easily confused class. Boxplots were also generated for each SAR variable which showed the median pixel values of each ocean cover across all available images.

When there is multiple testing, such as between 123 SAR variables, the probability of Type I error is inflated by the significance level to the power of the number of tests (Legendre and Legendre, 1998). To account for this, all the p-values where there was a pair-wise comparison were modified with the False Discovery Rate (FDR) correction (Benjamini and Hochberg, 1995) to reduce the chance of incorrectly accepting the null hypothesis.

In total, five SAR variables were selected, one for each ocean cover which had the highest minimum separability. The correlation of these five SAR variables was assessed using the Spearman rank correlation coefficient to examine colinearity between variables. The Spearman correlation coefficient was chosen to avoid the parametric assumptions (Hauke and Kossowski, 2011) as well as the assumptions of homoscedasticity (Ofungwu, 2014) of the Pearson coefficient of correlation.

\subsubsection{Direct Gradient Analysis}

Direct gradient analysis was used to assess the influence of SAR geometry and environmental variables on the SAR variables selected by THSD/G-H as well as others. Five matrices were defined. The RDA SAR Matrix contained the variables that were selected by the RDA forward-selection process. The Confounding Matrix included all the SAR geometry and environmental data (Appendix B) which were hypothesized to 
influence the SAR variables in such a way as to confound the relationship between the SAR variables and geophysical properties. A third matrix, called the Ocean Cover Identity Matrix, contained dummy-coded variables that indicated the ocean cover (meteoric, marine, MYI, FYI, water). A fourth matrix, called the Ice Island Identity Matrix, contained the dummy-coded names of the ice island (PII-B-1, WH1, WH2, PIIB-A, M1, M3, PII-B). The purpose of the latter matrix was to attempt to identify the effects of pseudo-replication of ice island targets in the RDA as well as to identify the importance of individual parent ice shelf characteristics upon the SAR variability. The final matrix was called the All SAR Matrix and contained the median pixel values for each feature of all 123 of the SAR variables. The purpose of this matrix was to test to see whether other SAR variables through forward selection could be used to explain the Ocean Cover Identity Matrix. The mismatch between ice classification and SAR image variable was zeroed for ice island samples as no time discrepancy existed due to the ice islands being classified directly in the SAR image itself. $\Phi$ was assigned to the mean of the other measures $\Phi$ for the generic ocean covers case as $\Phi$ only applies to ice island samples since those are the only samples which contained measured ridges. Furthermore, the ice island identity was zeroed for non-ice island samples.

To assess whether CCA or RDA was the more appropriate tool, both were performed using Canoco v4.53 (ter Braak and Smilauer, 2004) with similar centring and standardization. For RDA, SAR variables were centred and standardized as the measurement scales can differ between SAR variables (Šmilauer and Lepš, 2014). No centring or standardization to samples or transformations were applied. The scaling used 
was the default in CANOCO (scaling $=2$ ) and was focused on inter-variable correlations and SAR variable scores were computed by dividing by standard deviation. For CCA, scaling was focused on inter-SAR variable distances and biplot scaling was used. A 1000 iteration Monte-Carlo random unrestricted permutation approach was used to test the significance of the first canonical axis separately as well as all the axes combined to ensure the ordination was significant.

Using the direct gradient approach which explained the most variability, several questions were asked:

1. Which SAR variables from the All SAR Matrix explain the most variability in the Ocean Cover Identity Matrix?

2. What is the association of all variables from the Confounding Matrix on the RDA SAR variable matrix?

3. What is the relationship of all factors from the combined Confounding Matrix and the Ocean Cover Identity Matrix on the RDA SAR variable matrix?

4. What is the association of all factors from the Confounding Matrix and the Ocean Cover Identity Matrix on the RDA SAR variable matrix but with the effects of the Ice Island Identity matrix accounted for?

Question 1 was answered by considering the forward selection of the SAR variables as the independent variable and using the Ocean Cover Identity as the dependent variable. The ordination of these newly selected SAR variables could be compared to an ordination of the previously selected variables from THSD and G-H. To determine the optimal number of SAR variables, a scree plot was produced to assess how explained variance 
changed with added variables. Furthermore, the Spearman correlation coefficient was used to assess how similar the SAR variables were selected by each of the approaches. For Question 1, a 1000 iteration Monte-Carlo approach was used to test significance. Question 4 required a partial gradient analysis, where the variability due to the Ice Island Identity Matrix from Question 3 was removed. Questions 2 - 4 were answered based on qualitative assessment of the ordination triplots while Question 4 was answered based upon analysis of the forward selected SAR variables that explained the most variability in Ocean Cover Identity.

\subsubsection{SVM and SLIC}

SVM training and predicting was conducted using the library LibSVM v3.18 (Chang and Lin, 2011), which is an SVM library used to perform the SVM training and predicting. For SVM training, the median of each ocean cover computed for each SAR variable using a $0.5 \%$ random subsample of all pixels within that area. A single SAR variable was selected to classify each ocean cover as this was an exploratory initiative and a reduced, interpretable model was desired. Therefore, the SVM model was trained using the five best SAR variables from the THSD/G-H separability analysis. Using the weights in Table 3.6 to counter the greater occurrence of some ocean covers, and prescaling the input data to the range of $-1 \rightarrow+1$, the SVM model was trained using an RBF kernel. An RBF kernel was used due to its flexibility for non-linear decision rules however other kernels were not tested. 
Table 3.6: Weights given to classes during training of SVM models to reduce inherent imbalance of sampling. Weights are from $0 \rightarrow 1$ where a higher weight is given to classes with fewer samples.

\begin{tabular}{ccc}
\hline Ocean cover & Sample Counts & Weight \\
\hline MYI & 51 & 0.102 \\
FYI & 15 & 0.347 \\
Water & 14 & 0.372 \\
Meteoric & 65 & 0.080 \\
Marine & 53 & 0.098 \\
\hline
\end{tabular}

Weights were selected by normalizing the number of training samples of the respective ocean cover such that higher weights were given to rarer ocean covers (Table 3.6).

To optimize the SVM training parameters of $C$ and $\Upsilon$, an accurate classification would be needed across all images (Hsu et al., 2010). Consequently, a grid search for optimal $\mathrm{C}$ and $\Upsilon$ values was not possible, as good reference data were not available at the outset. The default LibSVM values of $C=1$ and $\Upsilon=1$ /number of input features was used (in the case of the SAR variable model, $\Upsilon=0.005$ ) as internally computed by LibSVM.

For classification on objects rather than on a per pixel basis, a SLIC superpixel segmentation of the $70 \mathrm{FQ}(\mathrm{W})$ images was undertaken using Pauli decomposition imagery from the combined T11, T22, T33 images. The Pauli decomposition was used as opposed to the five SAR variables identified by THSD/G-H due to a limitation in the SLIC implementation, which allows a maximum of three input variables. The SLIC segmentation routine used was from scikit-image v0.11dev (scikit-image developers, v0.11dev). The approximate number of segments was set to 1000 , smoothing kernel was set to 2 , compactness was set to 6 , minimum cluster was set to 0.1 and maximum cluster was set to 500. The minimum and maximum cluster sizes are the ratio to the average cluster size when considering the approximate number of segments in the image. The 
minimum cluster size is thus $1 / 10^{\text {th }}$ of the average cluster size while the maximum cluster size is equal to approximately 500 average clusters. The number of k-means iterations was set to 1000. Using the segments, the medians were extracted and classified according to the SVM model. The classified results were recombined into an image where the classification given to that particular median of the segment was assigned to all pixels in that segment. Nine FQ(W) SAR images that were withheld from the training procedure were then classified using the SVM classification model derived from the training images (Appendix D) in order to quantitatively and qualitatively assess classification accuracy when the images were completely independent from the training data. However, there were no marine ice islands in the nine test images so this assessment was only conducted for the other ocean covers.

To determine the accuracy of the classified images, the segments from the SLIC were assessed post-classification for their identity. The CIS charts from training were not used in the accuracy assessment as they are classified at a coarser scale than the classifications of this study. Furthermore, the time discrepancy between the SAR image and the CIS charts leads to a temporal mismatch. The extraction of median values from the features was sufficient for training; however, higher quality reference data were needed for accuracy assessment. CIS charts, Pauli decomposition images and Landsat-7 and 8 imagery were used to assess the ice conditions at the time of SAR image acquisition for the SLIC segments. 30 SLIC segments were randomly chosen for each image or whether the SAR image was a FQ or FQW. Further samples were added for each image to ensure the ice island feature was properly sampled. Since individual SLIC segments often 
comprised more than one ocean cover, the majority ocean cover was selected for accuracy assessment. Using this object-wise comparison, user's and producer's accuracies (Liu et al., 2007) were computed for each individual image, along with the Kappa coefficient (Stehman, 1996).

Based upon the results of the accuracy assessment, a further SAR variable was selected for each pair of ocean covers that had strong pairwise confusion. These SAR variables were selected based on the same maximum of the minimum difference of means as in 3.5.2.1, but instead this was a specific pair-wise difference of means. The correlation to the previous THSD/G-H selected SAR variables was computed using the Spearman rank coefficient as previously stated. 


\section{RESULTS}

\subsection{Descriptive Statistics}

\subsubsection{SAR Geometry and Environmental Data}

Histograms of all input variables showed that distributions were mostly non-normal. The histograms also indicated $\Phi$ occurred with near-uniform frequency. Temperature had a bimodal distribution, Wind speed was negatively skewed and $\theta$ was approximately normally distributed (Figure 4.1). The temperature ranged from $227 \mathrm{~K}$ to $278 \mathrm{~K}$, while wind speed ranged from 0 to $17 \mathrm{~m} \mathrm{~s}^{-1} . \theta$ ranged from to $19^{\circ}$ to $50^{\circ}$ and $\Phi$ ranged from $0^{\circ}$ to $90^{\circ}$ (Table 3.3). Image acquisitions dates began on 2009/09/15 and ended on 2012/01/26 and were generally limited to the winter months. 
a)
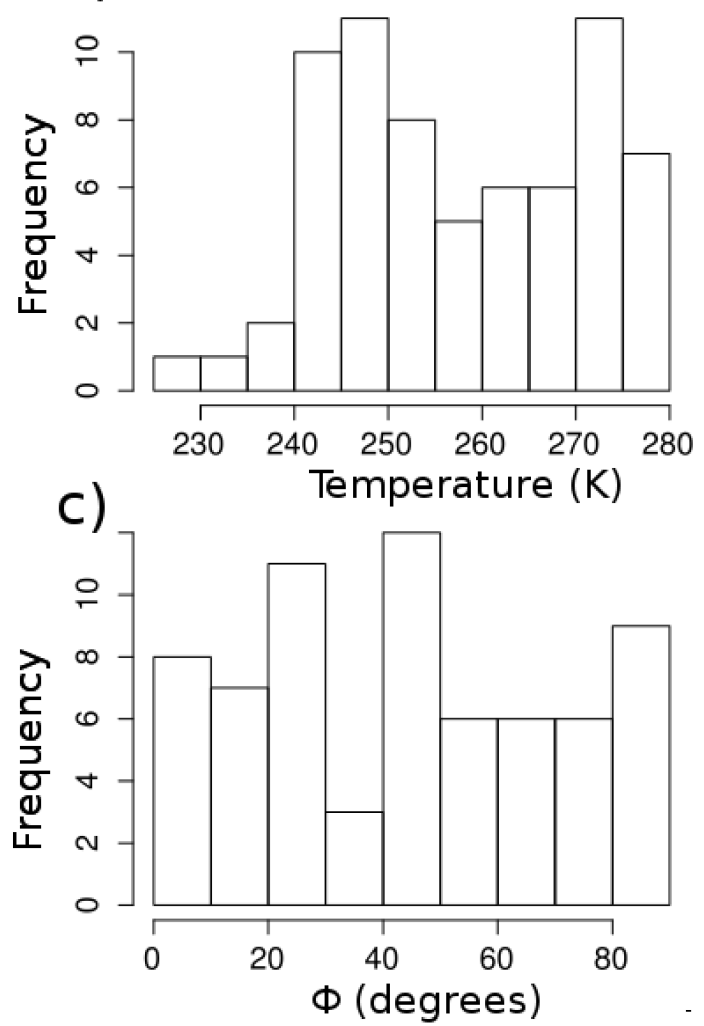

b)
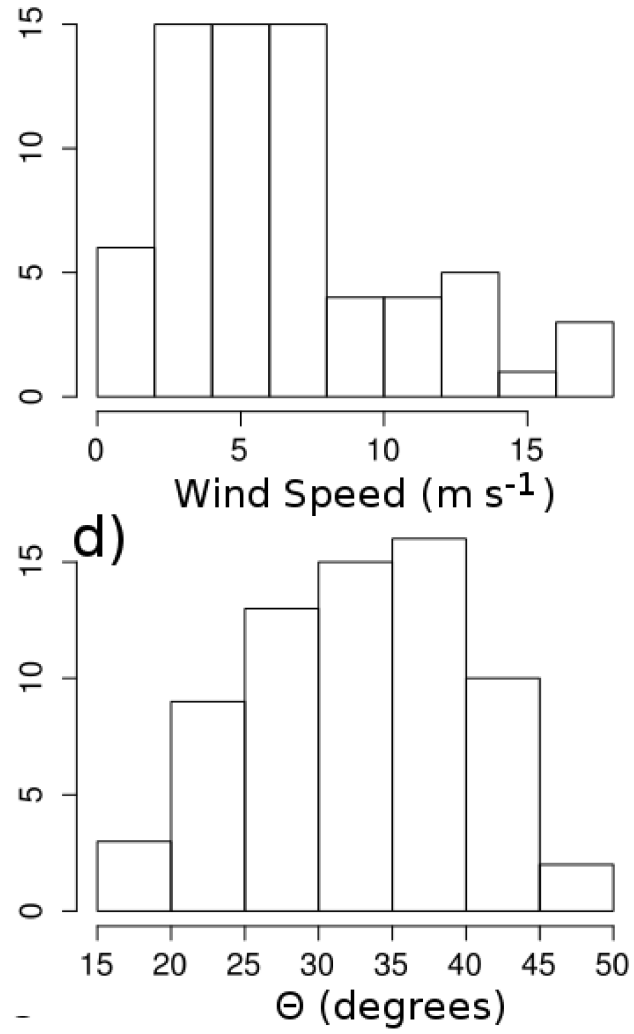

Figure 4.1: Histograms of explanatory variables. a) Temperature appeared bimodal b) wind speed was nearly normal c) $\Phi$ occurred with approximately equal frequency d) $\theta$ appeared normally distributed.

\subsection{SAR Variables}

$\sigma_{\mathrm{HH}}^{\mathrm{o}}$ ranged from -24.0 to $-5.6 \mathrm{~dB}, \sigma_{\mathrm{HV}}^{\circ}$ ranged from -35.1 to $-16.4 \mathrm{~dB}$ and $\sigma_{\mathrm{VV}}^{\circ}$ ranged from -22.2 to $-5.6 \mathrm{~dB}$ across all SAR images and ocean covers. The backscatter of individual ocean covers is summarized in Table 4.1. Water had the lowest backscatter due to the high degree of specular reflection, and meteoric ice had the highest backscatter due to the low salinity and high penetration depth leading to enhanced volume scattering. Meteoric ice and MYI had very similar backscatter in all polarizations while water and FYI were found to have similar backscatter. Meteoric ice had the highest variability in 


\section{backscatter except for $\sigma_{\mathrm{vv}}^{0}$.}

Table 4.1: Summary table of backscatter for ocean covers across all SAR. $\sigma_{V H}^{\circ}$ was not recorded as it is the same as $\sigma_{H V}^{\circ}$ due to the principal of reciprocity.

$$
\begin{array}{lll}
\sigma_{\mathrm{HH}}^{\circ}(\mathrm{dB}) & \sigma_{\mathrm{HV}}^{\circ}(\mathrm{dB}) & \sigma^{\circ}{ }_{\mathrm{VV}}(\mathrm{dB})
\end{array}
$$

\begin{tabular}{|c|c|c|c|c|c|c|c|c|c|c|c|c|c|c|c|}
\hline $\begin{array}{l}\text { Ocean } \\
\text { cover }\end{array}$ & 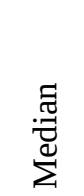 & $\sum_{\Sigma}^{\overparen{\Xi}}$ & $\begin{array}{l}\vec{\Delta} \\
\text { Dे } \\
\dot{\vec{D}}\end{array}$ & $\begin{array}{l}\text { 当 } \\
\text { 离 } \\
\text { 巻 }\end{array}$ & 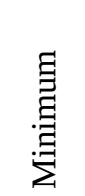 & 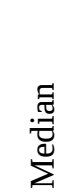 & $\sum_{\Sigma}^{\approx}$ & $\begin{array}{l}\overrightarrow{\vec{\Delta}} \\
\dot{\vec{n}}\end{array}$ & $\begin{array}{l}\text { 忍 } \\
\text { 离 } \\
\text { 巻 }\end{array}$ & 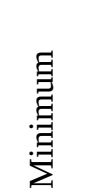 & 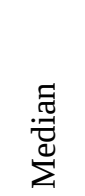 & $\sum_{\Sigma}^{\bar{\Xi}}$ & $\begin{array}{l}\dot{\vec{\Delta}} \\
\text { Dे } \\
\dot{\vec{n}}\end{array}$ & 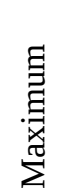 & $\begin{array}{l}\text { 罪 } \\
\text { 主 }\end{array}$ \\
\hline Water & -17.1 & -16.8 & 3.3 & -12.1 & -24.0 & -32.2 & -31.8 & 2.9 & -25.6 & -35.1 & -14.7 & -14.4 & 3.2 & -9.8 & -21.4 \\
\hline $\begin{array}{l}\text { Marine } \\
\text { Ice }\end{array}$ & -12.9 & -12.6 & 2.0 & -8.0 & -15.8 & -24.2 & -24.7 & 1.9 & -21.3 & -28.6 & -12.6 & -12.5 & 2.0 & -7.6 & -15.7 \\
\hline $\begin{array}{l}\text { Meteoric } \\
\text { Ice }\end{array}$ & -9.3 & -10.3 & 3.3 & -5.6 & -18.5 & -19.0 & -20.6 & 3.8 & -16.4 & -28.9 & -9.4 & -10.3 & 3.1 & -5.6 & -17.9 \\
\hline FYI & -16.0 & -16.5 & 3.2 & -9.5 & -21.8 & -31.1 & -30.4 & 3.4 & -21.4 & -35.2 & -16.0 & -16.8 & 3.3 & -9.5 & -22.2 \\
\hline MYI & -11.4 & -11.6 & 2.0 & -8.1 & -16.4 & -21.9 & -22.5 & 1.8 & -20.3 & -28.6 & -11.4 & -11.6 & 2.0 & -8.1 & -16.4 \\
\hline
\end{tabular}

To demonstrate the challenge of ice classification using backscatter alone, the ocean covers were also plotted as boxplots of median values in each image for $\sigma_{\mathrm{HH}}^{\circ}, \sigma_{\mathrm{HV}}^{\circ}$, and $\sigma_{\mathrm{VV}}^{\mathrm{o}}$ (Figure 4.2). 
a)

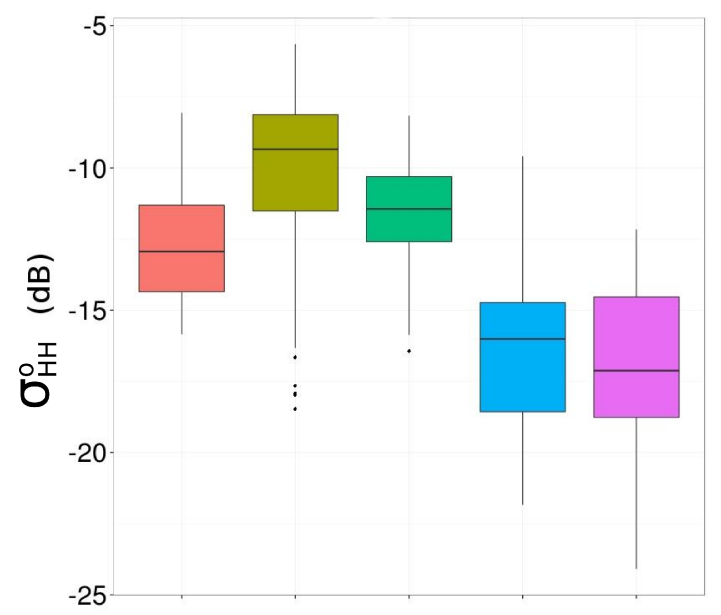

c)

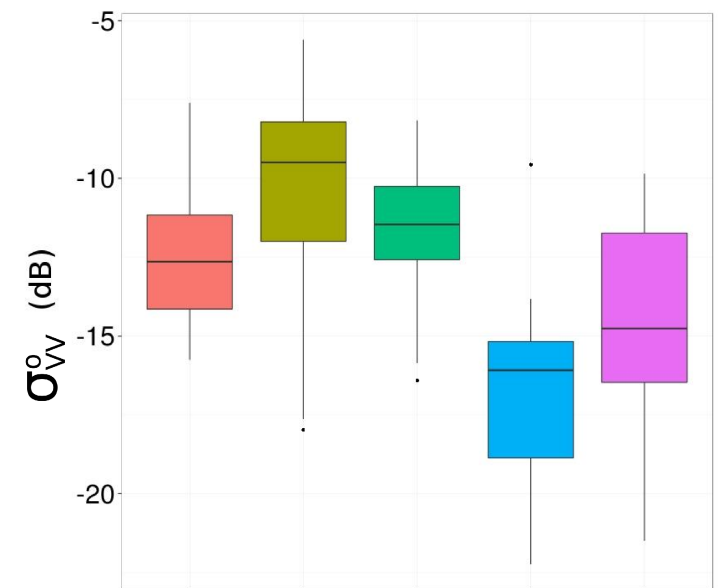

b)
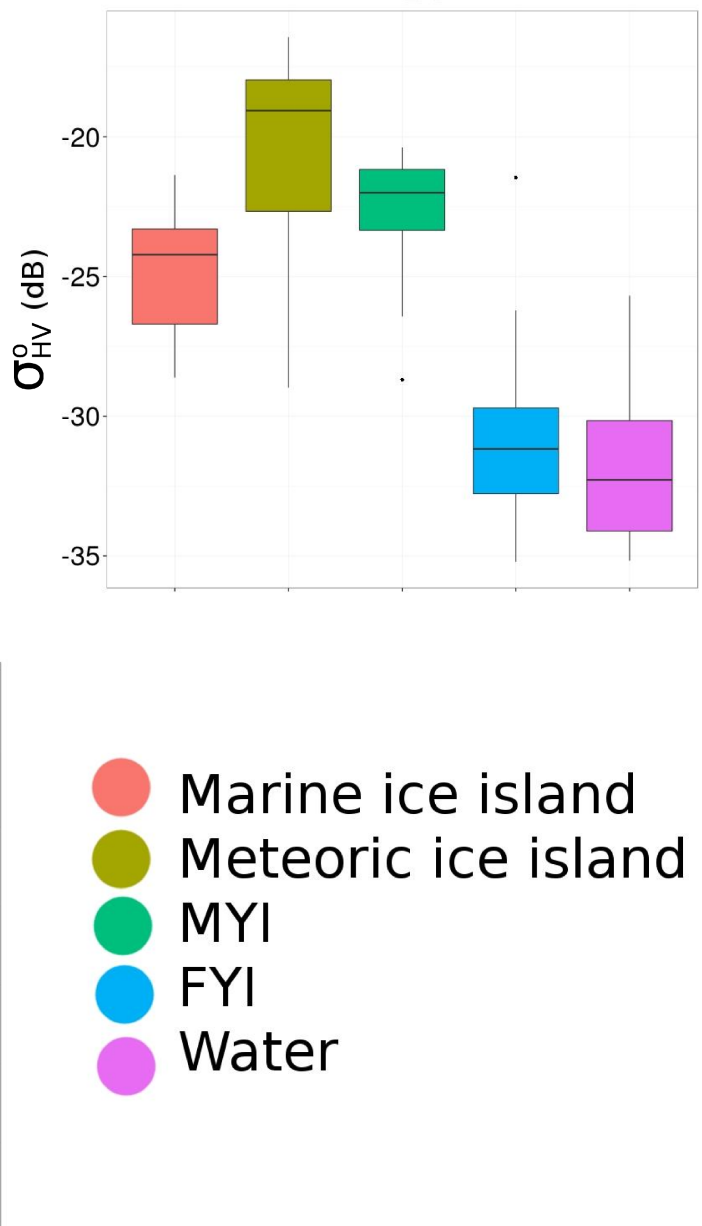

Figure 4.2: Boxplots of median backscatter in each image for each ocean cover. a) $\left.\sigma_{H V}^{\circ} b\right) \sigma_{H V}^{\circ}$ ) $\sigma_{\mathrm{VV}}$. The middle horizontal line represent the median, with the hinges representing the first and third quartiles, and the dots representing outliers.

In all three polarizations, there is little separability between the ocean covers and a high degree of overlap in distributions. The only exception is the case of marine ice in $\sigma^{\circ} \mathrm{HV}$ where some separability is evident to the other ocean covers. Since the boxplots are of the median backscatter for each ocean cover in each image and are already highly aggregated, the separability of ocean covers is likely even less than these boxplots demonstrate. 


\subsection{SAR Variable Selection}

\subsubsection{Assumptions}

To ensure that ANOVA, THSD and G-H are appropriate tools to assess difference of mean for selecting the most useful SAR variables, the assumptions of normality and heteroscedasticity were tested. It was found that greater than $73 \%$ of SAR variables had a p>0.05 in the Jarque-Bera test. In general, the SAR variables were mostly normal with near zero skewness and kurtosis. Levene test p-values demonstrated that most of the groups were heteroscedastic. The Levene test indicated that $95 \%$ of the comparisons have a heterogeneous distribution variance amongst groups. This heteroscedasticity violates an assumption of ANOVA/THSD, and confirmed the need for the G-H test as described earlier.

\subsubsection{Overall Separability of SAR Variables}

Ocean cover difference of means was determined for each SAR variable using ANOVA as a first assessment of group differences. In total, 100 SAR variables had a significant ANOVA p-value. The ANOVA scoring for all SAR variables is presented in Appendix C.

The SAR variables with the lowest separability had absolutely no separability between ocean covers and were often based upon phase, or imaginary components of SAR variable elements. For example, T13_imag is the $(1,3)$ imaginary component of the T3 coherency matrix and the boxplot showed minimal separability between the ocean covers.

The best five SAR variables with the most significant separability were 
Neumann_tau, reflectivity22ov11, reflectivity_11ov22, beta and entropy_shannon_P_norm each with a p-value of $<0.00001$. Most of these SAR variables were best at separating water from other ocean covers, such as reflectivity_22ov11, which is also known as the co-pol ratio $\left(\sigma_{\mathrm{VV}}^{\mathrm{o}} / \sigma_{\mathrm{HH}}^{\mathrm{o}}\right)$.

Since ANOVA identifies only if there is significant separability between groups, further analysis is needed to select the best SAR variables useful for maximizing separability of specific ocean covers. From the boxplots of median pixel values and using THSD/G-H, the top SAR variable for separating each ocean cover was selected. A summary of the THSD and G-H results is in Table 4.2. The best variables are given in Table 4.2 and the complete list for all ocean covers is given within Appendix D.

Table 4.2: Top SAR variables for separability of specific ocean covers based upon Maximum Base-Level Difference of Means Score with associated THSD and G-H p-values corrected with FDR.

\begin{tabular}{ccccc}
\hline Ocean Cover & SAR Variable & $\begin{array}{c}\text { Maximum Base- } \\
\text { Level } \\
\text { Difference of } \\
\text { Means Score } \\
\text { (unitless) }\end{array}$ & THSD p-value & G-H p-value \\
\hline Meteoric Ice & T22 & 0.258 & $<0.00001$ & $<0.00001$ \\
Marine Ice & Beta2 & 0.154 & 0.00226 & 0.00269 \\
MYI & Krogager_Kd & 0.128 & $<0.00001$ & $<0.00001$ \\
FYI & Beta & 0.130 & $<0.00001$ & $<0.00001$ \\
Water & Neumann_tau & 0.601 & $<0.00001$ & $<0.00001$ \\
\hline
\end{tabular}

Both the THSD and G-H p-values showed highly significant results for the pairwise difference of means (Table 4.2). Despite the heteroscedasticity, the THSD measured pvalue agreed with the G-H measured p-value but the G-H p-value was less significant. The separability was also visualized with boxplots of the distributions (Figure 4.3). The boxplots also suggest a linear classifier may not be the most appropriate. In Figure 4.3b where the marine ice distribution is in the middle between meteoric/MYI and FYI/water, 
non-linear classification is likely required much like in Figure 2.17.
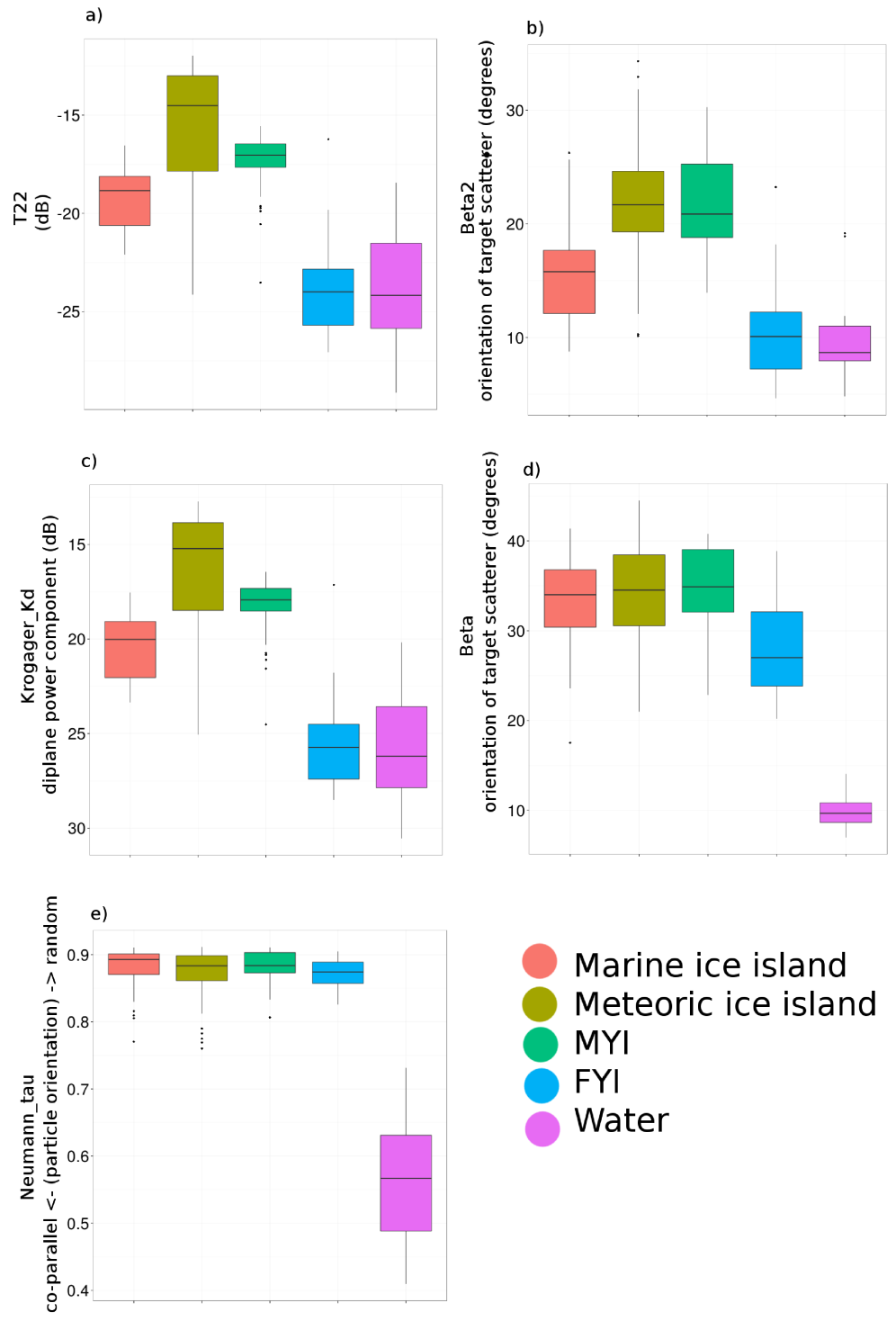

Marine ice island

Meteoric ice island

MYI

FYI

Water

Figure 4.3: Boxplot of ocean cover median values from each image for the most promising SAR variables at separating specific ocean covers. a) T22 for meteoric ice separability b) Beta2 for marine ice separability c) Krogager_Kd for MYI ice d) Beta for FYI separability e) Neumann_tau for water separability.

The correlations between the THSD/G-H selected SAR variables were plotted. The pairs of Beta/Neumann_tau and T22/Krogager_Kd had strong correlations while the remaining SAR variables had much lower correlations. The correlation between some SAR variables may lead to confusion or diminished separability over less correlated SAR 
variables. However, in these SAR variables, the separability between respective ocean covers was still measurable so the correlation of SAR variables may not be important in a classifier.

\subsection{Direct Gradient Analysis}

\subsubsection{Selecting CCA or RDA and Significance tests}

The comparison of total explained variance between CCA and RDA was performed and is shown in Table 4.3. Both techniques performed about equally across the variables and for Questions 1 and 2. RDA was able to explain more variance than CCA in Questions 3 and 4. It was therefore selected for all further analysis. RDA significance was 0.001 for all ordination axes and all ordination questions, including the all SAR variables case and THSD/G-H selected variable case of Question 1.

Table 4.3: Comparing explained variance (\%) for the CCA and RDA ordinations for the four RDA experiments

\begin{tabular}{|c|c|c|c|c|c|c|c|}
\hline & & & Question 1 & & Question 2 & Question 3 & Question 4 \\
\hline & & $\begin{array}{c}\text { All SAR } \\
\text { variables }\end{array}$ & $\begin{array}{c}12 \text { RDA } \\
\text { selected } \\
\text { SAR } \\
\text { variables }\end{array}$ & $\begin{array}{c}\text { THSD/G- } \\
\text { H selected } \\
\text { SAR } \\
\text { variables }\end{array}$ & & & \\
\hline \multirow{2}{*}{ 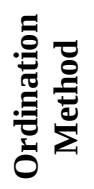 } & CCA & 92.2 & 65.5 & 44.4 & 23.3 & 42.0 & 41.9 \\
\hline & RDA & 92.1 & 64.7 & 43.2 & 23.0 & 61.7 & 67.6 \\
\hline
\end{tabular}




\subsubsection{Direct Gradient Analysis of RDA Selected SAR Variables}

Question 1 - Which SAR variables from the All SAR Matrix explain the most variability in the Ocean Cover Identity Matrix?

It was found that the four axes explained similar amounts of variation when using all SAR variables (Table 4.4). This was expected and suggests that the relationships between the SAR variables are complex with so many variables. The best five SAR variables were A12, beta3, I12, Krogager_Kh, Neumann_tau. Together, these explained $47.7 \%$ of the variability in ocean cover. Only Neumann_tau was also selected by the THSD/G-H method. A12 is the cross-polarized amplitude, beta3 is the 3rd beta angle, I12 is the crosspolarized intensity and Krogager_Kh is the helical component of the Krogager decomposition.

Table 4.4: Cumulative amount of variance explained by the 1st, 2nd, 3rd and 4th axes using all SAR variables.

\begin{tabular}{lcccc}
\hline & Axis 1 & Axis 2 & Axis 3 & Axis 4 \\
\hline $\begin{array}{l}\text { Cumulative } \\
\begin{array}{l}\text { Amount of } \\
\text { Variance } \\
\text { Explained (\%) }\end{array}\end{array}$ & 27.0 & 50.8 & 72.7 & \\
\hline
\end{tabular}

Figure 4.4 contains the correlation matrix comparing the THSD/G-H as well as the RDA SAR variables with the absolute Spearman correlation coefficient. Strong correlations were found between Krogager_Kd/Krogager_Kh, T22/I12, T22/Krogager_Kh, beta2/beta3, Krogager_Kd/A12 and T22/A12. These strong correlations suggest that similar geophysical differences and their effects on the electromagnetic waves are being used to aid in separability. Furthermore in the RDA variable group, strong correlations were found between A12/I12, Krogager_Kh/I12 and Krogager_Kh/A12. 


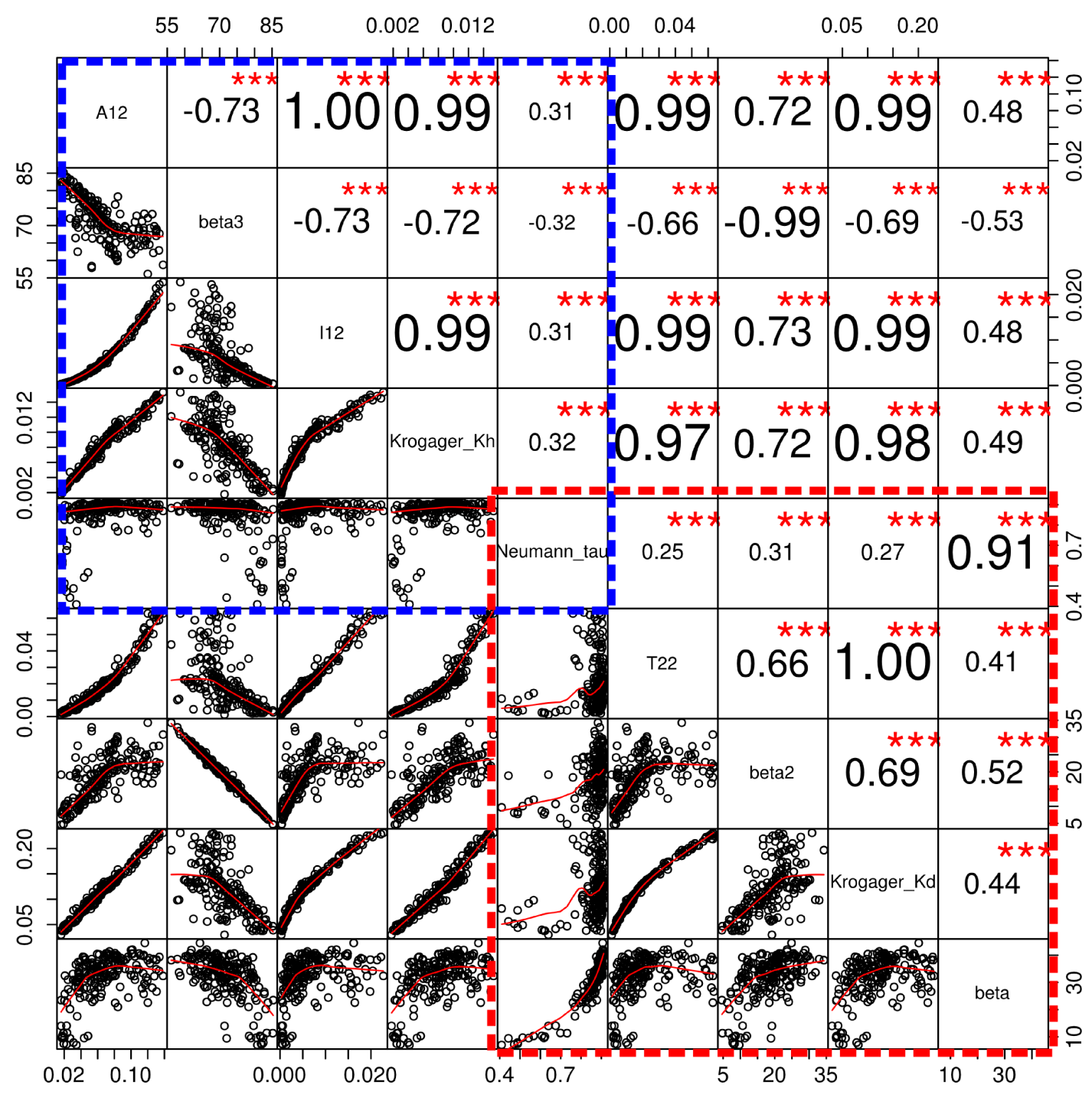

Figure 4.4: Correlation matrix of median values for five THSD/G-H and five RDA selected SAR variables. The THSD/G-H SAR variables are in red and the RDA selected SAR variables are in blue. Absolute Spearman correlation coefficients are given. ${ }^{* * *}$ signifies a highly significant correlation of $p \leq 0.001$. The red line is a fitted function.

The five SAR variables selected with THSD/G-H explained 43.2\% of the variability in ocean cover identity while the five RDA selected variables explained $47.7 \%$. The forward selection of SAR variables in RDA was found to explain slightly more variability than the THSD/G-H approach. This difference suggests that although the THSD/G-H approach 
selects individual SAR variables that are significant in pairwise comparison, it does not account for complex multivariate variability. This complex multivariate variability is not accounted for with basic difference of means measures since those are assessing differences in distributions with no regard for variability patterns. Figure 4.5 contains a screeplot of the cumulative explained variance of the first 35 SAR variables selected using automated forward selection and the associated p-values for each variable in a model containing the cumulative SAR variables. The screeplot is too continuous to define a single elbow, but the point of maximum curvature is approximately around the $10^{\text {th }}$ to $16^{\text {th }}$ SAR variable, suggesting parsimonious explanation of the ocean cover identity may be achievable with the prior 10 to 16 SAR variables. Approximately the first 21 SAR variables can be considered the limit to significant explained variability as above this, the p-values become unstable and increase above 0.05 significance level. 


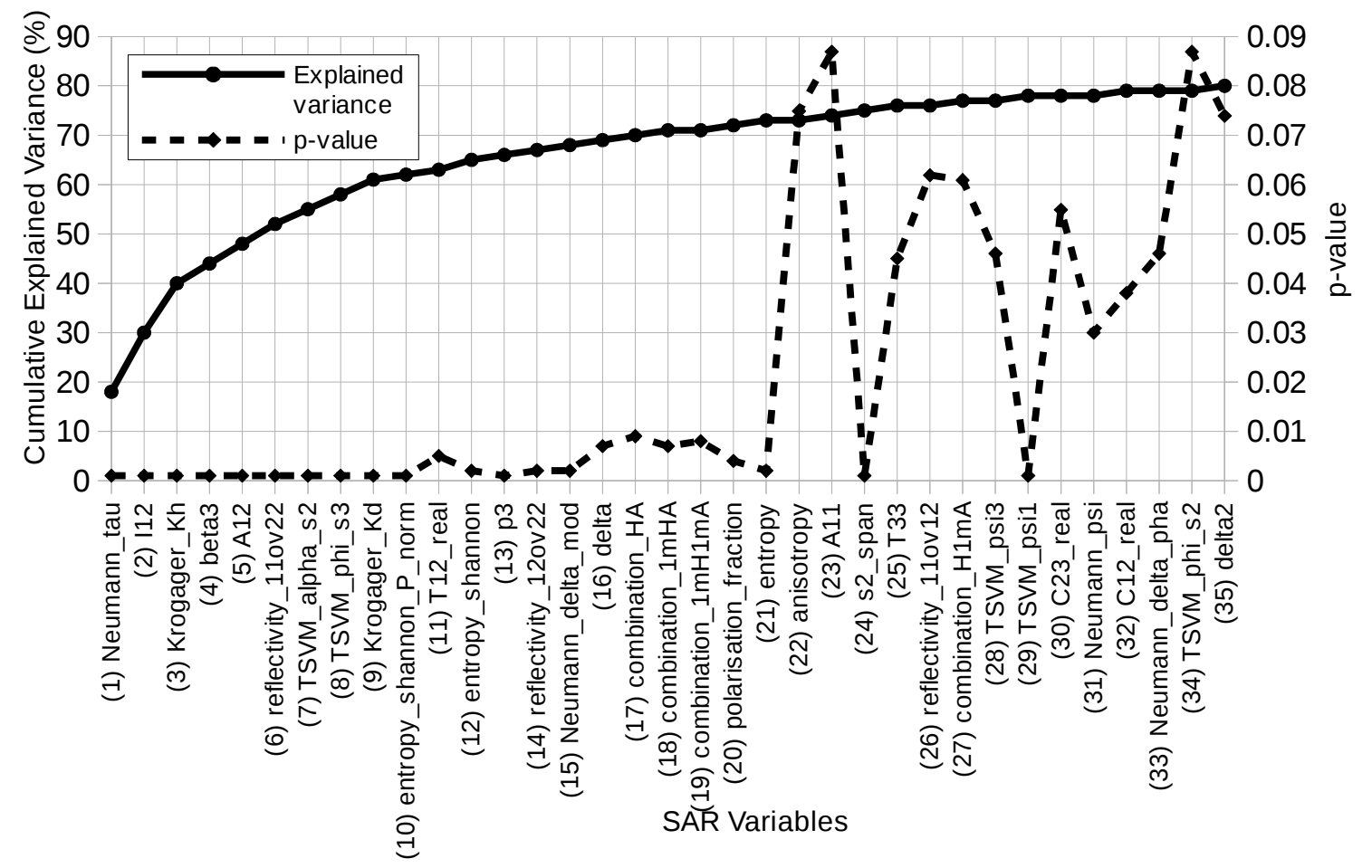

Figure 4.5: Screeplot of cumulative explained variance of Monte-Carlo forward selected SAR variables.

Based on the estimated point of maximum curvature, the first 12 RDA selected SAR variables were chosen. These were: Neumann_tau, I12, Krogager_Kh, beta3, A12, reflectivity_11ov22, TSVM_alpha_s2, $\quad$ TSVM_phi_s3, $\quad$ Krogager_Kd, entropy_shannon_P_norm, T12_real, and entropy_shannon. To compare the five SAR variables selected with THSD/G-H and the 12 SAR variables selected with RDA, the triplots of Figure 4.6 and Figure 4.7 were produced. It was found that the explained variation was similar between all the axes, where Axis 1 and 2 explained similar amounts of variation while Axis 3 and 4 explained about half the variation of Axis 1 and 2. Axis 1 and 2 combined explain about $62.4 \%$ of the total explainable variation (Table 4.5). The total explained variance with 12 SAR variables is $64.7 \%$. 
Table 4.5: Cumulative amount of variance explained by the 1st, 2nd, 3rd and 4th axes for the 12 forwardselected SAR variables.

\begin{tabular}{|c|c|c|c|c|}
\hline & Axis 1 & Axis 2 & Axis 3 & Axis 4 \\
\hline $\begin{array}{l}\text { Cumulative } \\
\text { Amount of } \\
\text { Variance } \\
\text { Explained (\%) }\end{array}$ & 20.7 & 40.4 & 52.8 & 64.7 \\
\hline
\end{tabular}

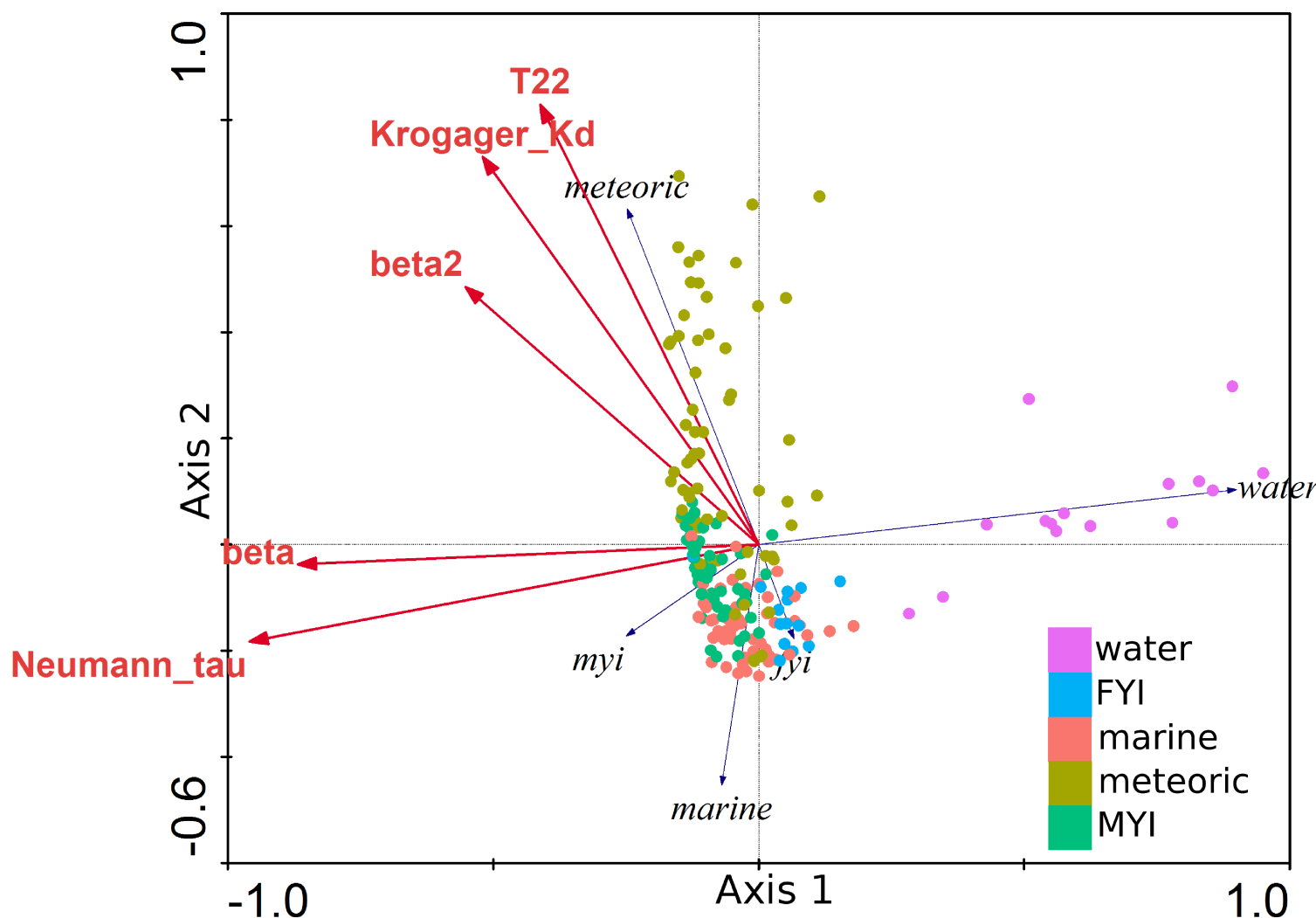

Figure 4.6: RDA triplot of THSD/G-H selected SAR variables to explain ocean cover identity. The coloured dots represent the different ocean covers in the ordination space. 


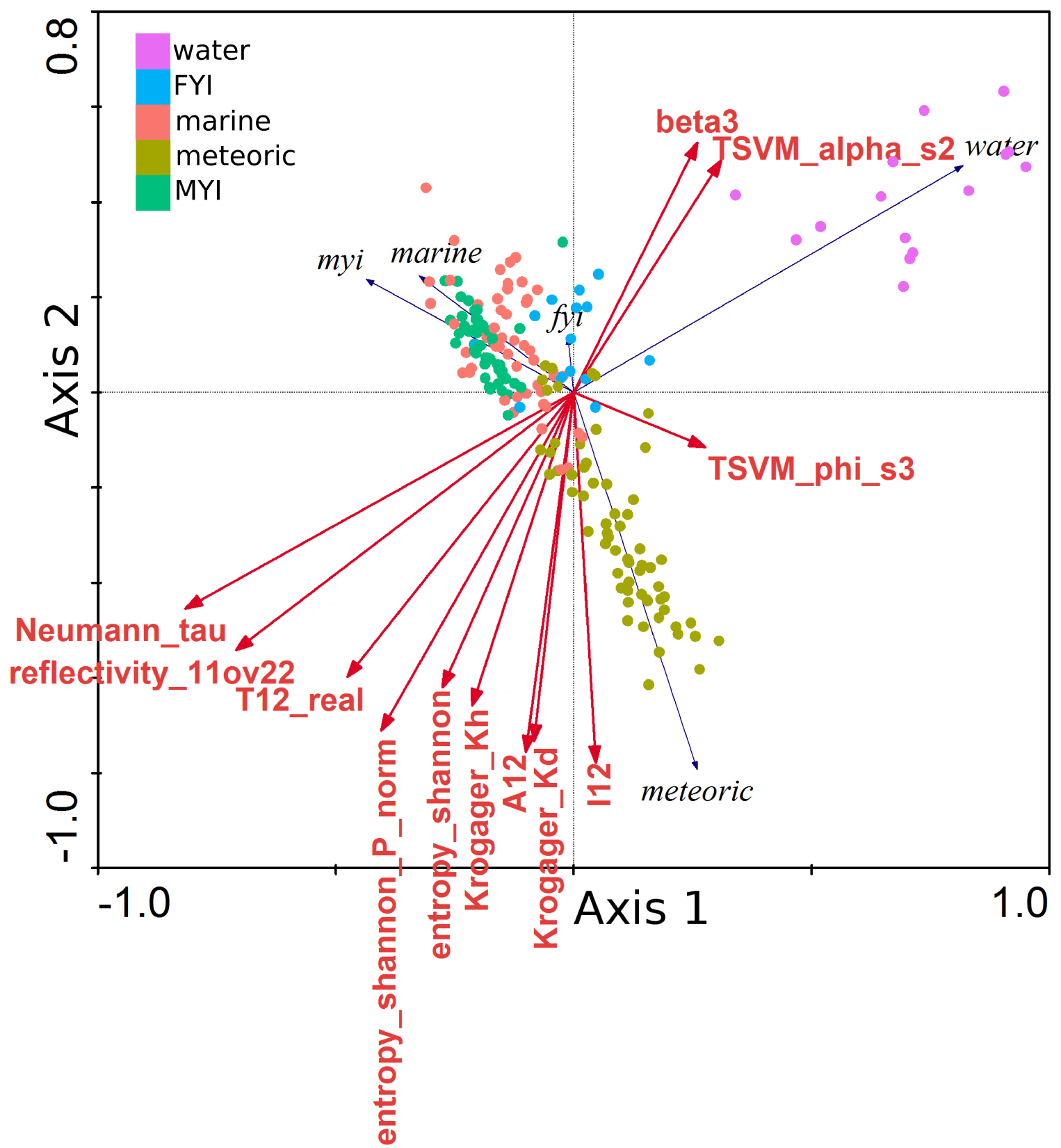

Figure 4.7: RDA triplot of RDA selected SAR variables to explain ocean cover identity. The coloured dots represent the different ocean covers in the ordination space.

Question 2 - What is the association of all variables from the Confounding Matrix on the RDA SAR variable matrix?

It was found that the primary gradients were on Axis 1, which explained $18.7 \%$ of the variability in the RDA selected variables, or $73.9 \%$ of the total explainable variance 
(Table 4.6). In the RDA triplot in Figure 4.8, T12_real, reflectivity_11ov22 and entropy_shannon_P_norm were found to be strongly negatively correlated with temperature and somewhat with wind. TSVM_phi_s3 and TSVM_alpha_s2 were positively correlated with temperature. Variables such as Krogager_Kd, I12, A12, Krogager_Kh, Neumann_tau and entropy_shannon were moderately negatively correlated to $\theta$ and temperature but were strongly correlated to the temporal offset between the RS2 FQ(W) and CIS reference chart. None of these SAR variables correlated with $\Phi$. Beta3 was similarly positively correlated with offset, and somewhat positively correlated with $\theta$, temperature and wind. It was difficult to assess how the confounding factors influenced the SAR variables as the effects of the ocean cover identity were not plotted, so correlations between ocean covers, SAR variables and possible geophysical explanations were not apparent.

Table 4.6: Cumulative amount of variance explained by the 1st, 2nd, 3rd and 4th axes in the RDA analysis to understand the association of all variables from the Confounding Matrix on the RDA SAR variable matrix.

\begin{tabular}{lcccc}
\hline & Axis 1 & Axis 2 & Axis 3 & Axis 4 \\
\hline $\begin{array}{l}\text { Cumulative } \\
\begin{array}{l}\text { Amount of } \\
\text { Variance }\end{array}\end{array}$ & 18.7 & 21.2 & & \\
Explained (\%) & & & 22.5 & 23.0 \\
\hline
\end{tabular}




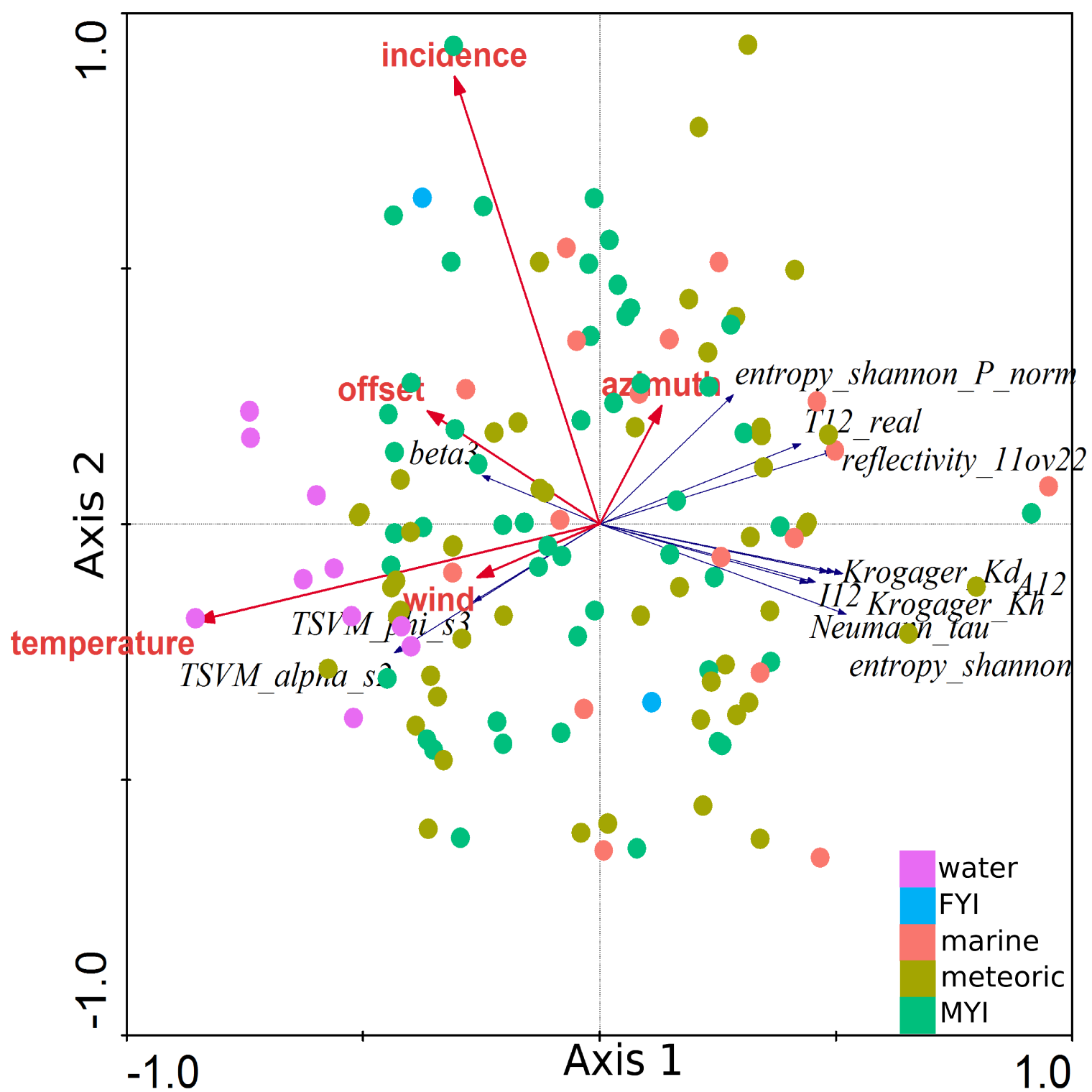

Figure 4.8: RDA triplot attempting to explain the RDA selected SAR variables with the confounding factors. The coloured dots represent the different ocean covers in the ordination space.

Question 3 - What is the relationship of all factors from the combined Confounding Matrix and the Ocean Cover Identity Matrix on the RDA SAR variable matrix?

It was noted that the amount of variance explained on Axis 1 was 46.6\%. Axis 1 contained $73.5 \%$ of the total explainable variance (Table 4.7). In total, Axis 4 explained $61.7 \%$ of the variance. Figure 4.9 contains the RDA triplot using all confounding 
variables as well as ocean cover identity to explain the SAR variables. All ocean cover variables except MYI explained more variability in the SAR variables than the confounding factors. This suggests that classification of the ocean covers may be achievable with the appropriate methodology as the confounding factors were found to be less important than ocean cover identity. Variables such as Neumann_tau, reflectivity_11ov22, T12_real and, to a lesser degree, entropy_shannon_P_norm were able to enhance separability of water from the other ocean covers as these variables correlate strongly to maximizing the distance between water and the ice types. Variables such as entropy_shannon, Krogager_Kh, A12, Krogager_Kd and T12 and, to a lesser degree beta3, TSVM_alpha_s2 and TSVM_phi_s3 were able to separate the ice ocean covers from each other as they correlated to maximizing the distance between the ice covers. Water was very strongly correlated with Neumann_tau, reflectivity_11ov22, T12_real and, to some extent, with entropy_shannon_P_norm. The various ice ocean covers strongly correlated to a combination of entropy_shannon, Krogager_Kh, A12, Krogager_Kd and I12. Since the variable space is multivariate with many SAR variables, it is difficult to understand why these SAR variables are related to the confounding variables.

Table 4.7: Cumulative amount of variance explained by the 1st, 2nd, 3rd and 4th axes in the RDA analysis to understand the relationship of all factors from the combined Confounding Matrix and the Ocean Cover Identity Matrix on the RDA SAR variable matrix.

\begin{tabular}{lcccc}
\hline & Axis 1 & Axis 2 & Axis 3 & Axis 4 \\
\hline $\begin{array}{l}\text { Cumulative } \\
\text { Amount of } \\
\begin{array}{l}\text { Variance } \\
\text { Explained (\%) }\end{array}\end{array}$ & 46.6 & 57.3 & & \\
\hline
\end{tabular}




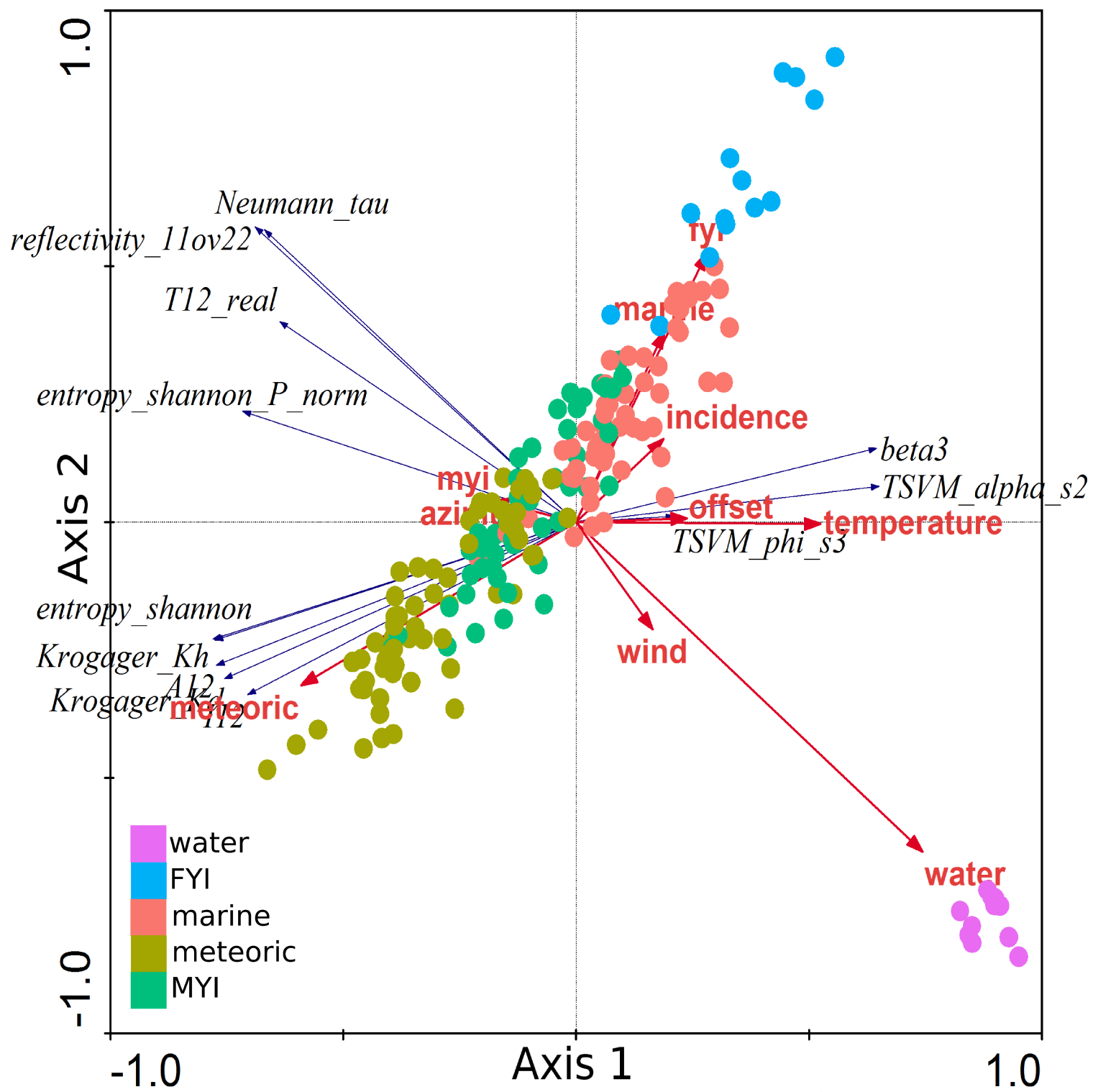

Figure 4.9: RDA triplot explaining the RDA selected SAR variables with the confounding factors and the ocean cover identity. The coloured dots represent the different ocean covers in the ordination space.

Question 4 - What is the association of all factors from the Confounding Matrix and the Ocean Cover Identity Matrix on the RDA SAR variable matrix but with the effects of the Ice Island Identity matrix accounted for?

The previous ordination explained $61.7 \%$ of the variance in the SAR variables. However, when the influence of ice island identity was removed, the ordination was able to explain a $67.6 \%$ of variation (Tables 4.7 and 4.8 ). This implies that the individual ice 
islands are, to some degree, unique in their SAR variable response. It also suggests that pseudo-replication, (the repeated resampling of individual ice islands which violates the assumption of independence) does influence the ordination somewhat. However, given that the amount of variation is small, it likely does not unduly influence the ordination results.

Table 4.8: Cumulative amount of variance explained by the 1st, 2nd, 3rd and 4th axes in the RDA analysis to understand the association of all factors from the Confounding Matrix and the Ocean Cover Identity Matrix on the RDA SAR variable matrix but with the effects of the Ice Island Identity matrix accounted for?

\begin{tabular}{lcccc}
\hline & Axis 1 & Axis 2 & Axis 3 & Axis 4 \\
\hline $\begin{array}{l}\text { Cumulative } \\
\begin{array}{l}\text { Amount of } \\
\text { Variance }\end{array}\end{array}$ & 50.4 & 63.4 & & \\
Explained (\%) & & & 66.0 & 67.6 \\
\hline
\end{tabular}

\subsection{SVM Classification}

Overall the classifications of test images had poor accuracy and failed to detect ice islands. The following assessments explore the classifications qualitatively and quantitatively to understand where the segmentation and classification may have failed. Figures 4.10, 4.11 and 4.12 are examples of the test image classifications and show the classified images with the known ocean covers, the SLIC segmentation regions, as well as SAR variable model classifications. Tables 4.9, 4.10 and 4.11 are presented for individual accuracy assessment based on the images selected for the qualitative image assessment. Appendix E contains the individual classification accuracy for all nine test images. 


\subsubsection{Classification Accuracy Assessment}

Figure 4.10a is a FQ17W image acquired 2012/10/29-21:46:43 185 km southeast of Clyde River of a meteoric ice island surrounded by open water. The SLIC segmentation in Figure 4.10b shows some adherence to boundaries of the ice island to the east. In Figure 4.10c, most of the image was correctly classified as water, except for perhaps small ice island fragments which were below the minimum size threshold $1 / 10^{\text {th }}$ average cluster size in the SLIC. The ice island, however, was incorrectly split between MYI and FYI. 

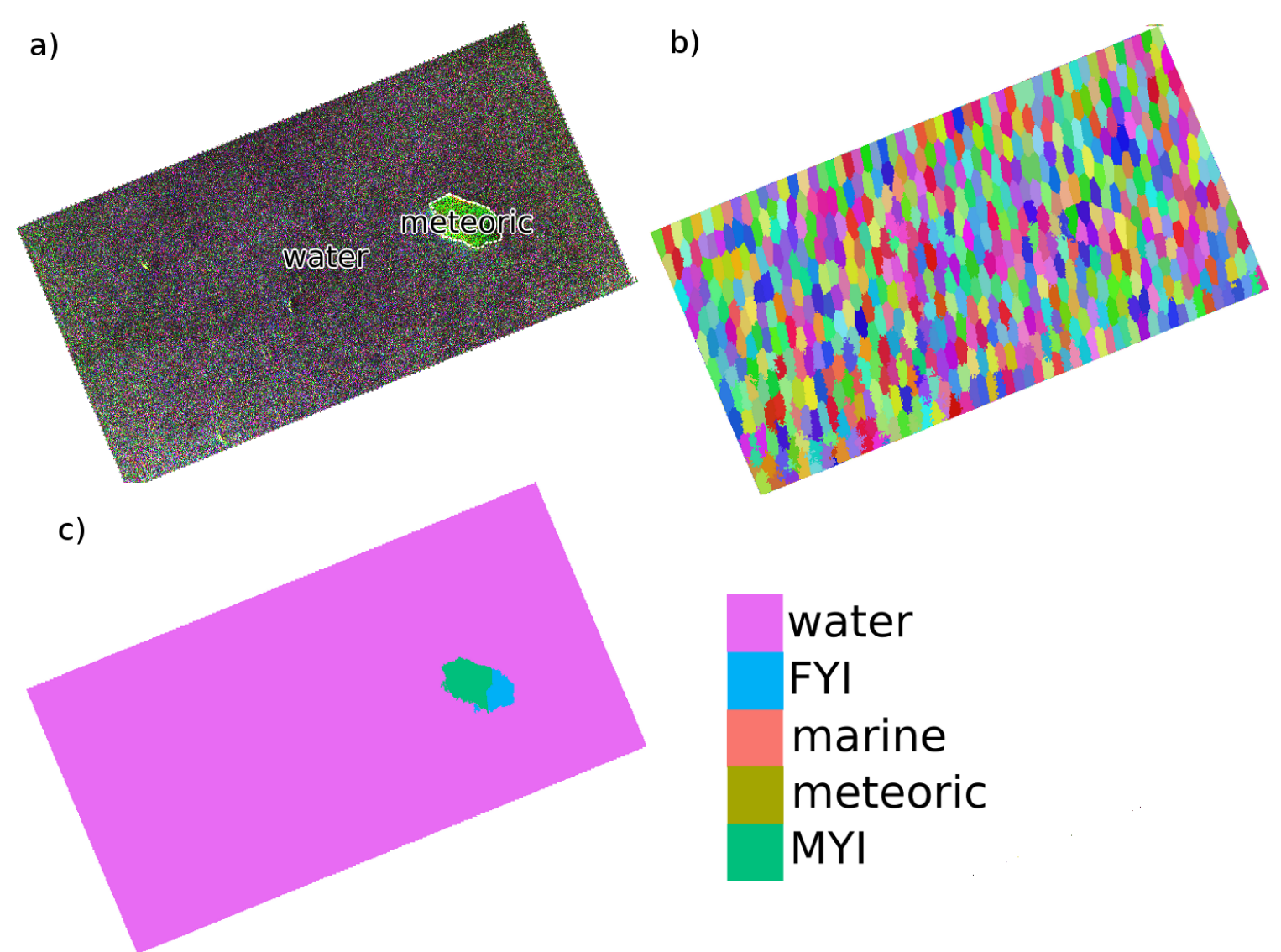

Figure 4.10: RS2 FQ17W image acquired 2012/10/29-21:46:43 $185 \mathrm{~km}$ southeast of Clyde River. a) Pauli decomposition, showing open water and a meteoric ice island fragment. b) SLIC segmentation of the Pauli decomposition where each colour represents a unique object. c) SAR variable classification using Beta, Krogager_Kd, Neumann_tau, Beta2 and T22 for FYI, MYI, water, marine and meteoric ice. Most of the image was correctly classified as water, except for perhaps the small ice island fragments which were below the minimum threshold in the SLIC. The ice island however was incorrectly split between MYI and FYI.

Table 4.9 contains the accuracy assessment for the image acquired on 2012/10/2921:46:43 (Figure 4.10). Water was very accurately classified while meteoric ice was misclassified as FYI or, more commonly, MYI. Overall, there was moderate agreement (on chance-adjusted measures for accuracy assessment in remote sensing image classification). 
Table 4.9: Accuracy assessment for RADARSAT-2 FQ17W image acquired 2012/10/29-21:46:43. Kappa was 0.42 .

\begin{tabular}{|c|c|c|c|c|c|c|c|}
\hline & \multicolumn{4}{|c|}{ Reference Data } & \multirow[b]{2}{*}{ Total Segments } & \multirow{2}{*}{ User Accuracy (\%) } \\
\hline & & water & FYI & meteoric & MYI & & \\
\hline \multirow{4}{*}{ 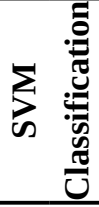 } & water & 29 & 0 & 0 & 0 & 29 & 100 \\
\hline & $\overline{\text { FYI }}$ & 0 & 0 & 3 & 0 & 3 & 0 \\
\hline & meteoric & 0 & 0 & 0 & 0 & 0 & 0 \\
\hline & MYI & 0 & 0 & 7 & 0 & 7 & 0 \\
\hline \multicolumn{2}{|c|}{ Total Segments } & 29 & 0 & 10 & 0 & 39 & \\
\hline \multicolumn{2}{|c|}{ Producer Accuracy (\%) } & 100 & 0 & 0 & 0 & & \\
\hline
\end{tabular}

Figure 4.11 is a FQ15W image acquired of the meteoric PII-B-1 ice island fragment 2012/06/14-21:42:37 145 km southeast of Clyde River. Much of the image is FYI with a meteoric ice island fragment embedded in the landfast ice with FYI pack right edge beyond a narrow lead. There was a new ice in the eddy downstream of the grounded ice island to the bottom of the image (Figure 4.11a). Figure 4.11b shows the SLIC segmentation where the boundary adherence, especially around the lead, is poor. Some of the scene was incorrectly classified, in particular the FYI and water to the bottom of the image. The larger FYI floes were correctly classified, however, there is incorrectly classified MYI in the classification to the right portion of the classification. There is an odd FYI misclassified region in the lead. There were also no areas classified as ice islands in the resulting classification, possibly because the appearance of the ice island in this image was different from other images, perhaps due to the mid-June image acquisition where the temperature was likely above freezing. 

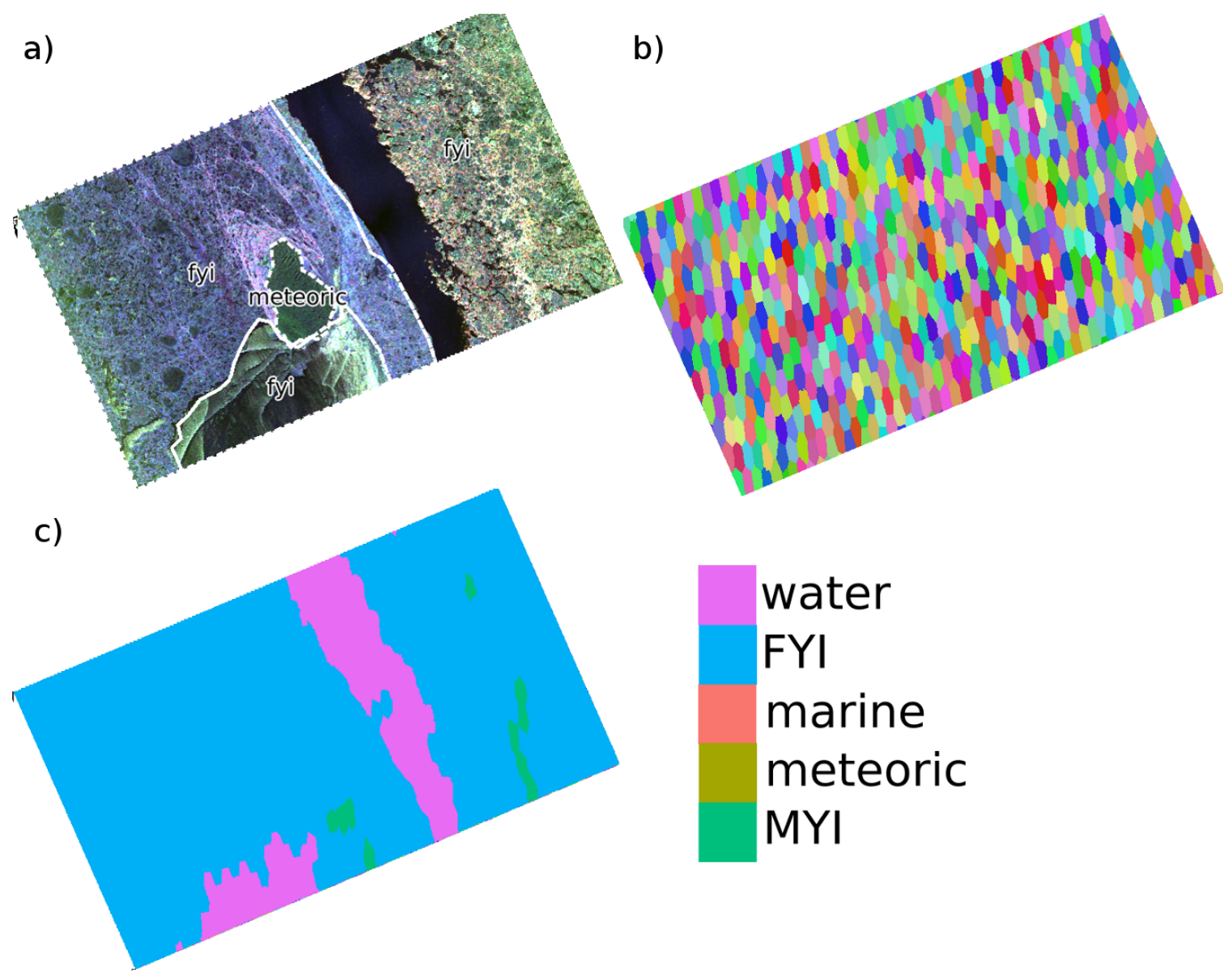

marine meteoric MYI

Figure 4.11: RS2 FQ15W image acquired of PII-B-1 2012/06/14-21:42:37 145 km southeast of Clyde River. a) Pauli decomposition, showing FYI in different formations and the PII-B-1 meteoric ice island fragment. b) SLIC segmentation of the Pauli decomposition where each colour represents a unique object c) five SAR variable classification using Beta, Krogager_Kd, Neumann_tau, Beta2 and T22 for FYI, MYI, water, marine and meteoric ice. The lead is largely correctly classified, however there is strong confusion on the smooth FYI to the bottom with water and some MYI/FYI confusion on the FYI flow to the right of the image.

Table 4.10 presents the accuracy assessment for the 2012/06/14-21:42:37 image (Figure 4.11). In this image, there was slight confusion of water with FYI, and slight confusion of FYI with MYI. There was much stronger confusion of meteoric ice where it was entirely classified as FYI. These confusions led to much better producer accuracy than user accuracy for FYI. Due to the lack of classification of the meteoric ice that was present, its user and producer accuracies were 0. Overall, the Kappa suggests marginally 
fair agreement.

Table 4.10: Accuracy assessment for RADARSAT-2 FQ15W image acquired 2012/06/14-21:42:37. Kappa was 0.21 .

\begin{tabular}{|c|c|c|c|c|c|c|c|}
\hline & \multicolumn{4}{|c|}{ Reference Data } & \multirow{2}{*}{ Total Segments } & \multirow{2}{*}{ User Accuracy (\%) } \\
\hline & & water & FYI & meteoric & MYI & & \\
\hline \multirow{4}{*}{ 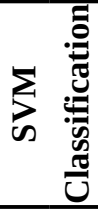 } & water & 4 & 0 & 0 & 0 & 4 & 100 \\
\hline & FYI & 1 & 24 & 17 & 0 & 42 & 57.1 \\
\hline & meteoric & 0 & 0 & 0 & 0 & 0 & 0 \\
\hline & MYI & 0 & 1 & 0 & 0 & 1 & 0 \\
\hline \multicolumn{2}{|c|}{ Total Segments } & 5 & 25 & 17 & 0 & 47 & \\
\hline \multicolumn{2}{|c|}{ Producer Accuracy (\%) } & 80.0 & 96.0 & 0 & 0 & & \\
\hline
\end{tabular}

Figure 4.12a shows an image acquired 2013/04/08-21:50:38 that contains an unknown meteoric ice island fragment and FYI. Figure 4.12b contains the SLIC segmentations, which do not seem to delineate any of the particularly interesting FYI floes or the distinct boundaries of the ice island. Figure 4.12c contains the SAR SVM classification. This classification found some MYI floes, which was not entirely unexpected as the CIS charts suggested some trace MYI. The image is largely classified correctly as FYI, however the ice island is not detected at all. 


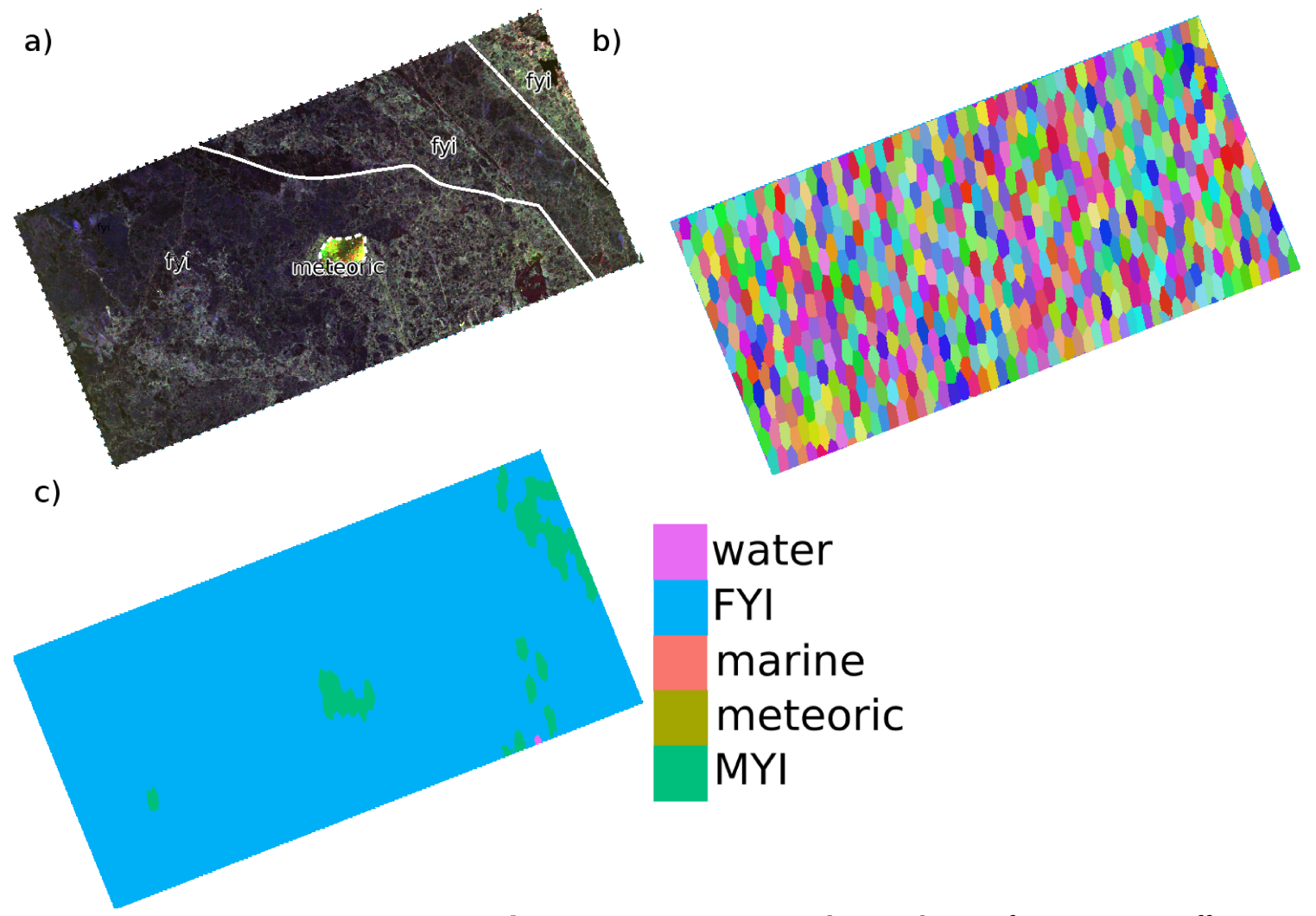

Figure 4.12: RS2 FQ19W image acquired 2013/04/08-21:50:38 90 km southeast of Home Bay, Baffin Island. a) Pauli decomposition, showing FYI in different formations and an unknown meteoric ice island fragment. b) SLIC segmentation of the Pauli decomposition where each colour represents a unique object c) five SAR variable classification using Beta, Krogager_Kd, Neumann_tau, Beta2 and T22 for FYI, MYI, water, marine and meteoric ice. The FYI is largely correctly classified, however the ice island is not correctly classified as meteoric ice.

Table 4.11 contains the accuracy assessment for the 2013/04/08-21:50:38 SAR image (Figure 4.12). In this image, the FYI was very accurately classified with one exception where there was some confusion with MYI. Similarly to all other images, meteoric ice was not classified at all and instead was misclassified as MYI and FYI. Due to the strong classification of FYI, the Kappa statistic suggested fair agreement. 
Table 4.11: Accuracy assessment for RADARSAT-2 FQ15W image acquired 2013/04/08-21:50:38. Kappa was 0.35 .

\begin{tabular}{|c|c|c|c|c|c|c|c|}
\hline & \multicolumn{4}{|c|}{ Reference Data } & \multirow{2}{*}{ Total Segments } & \multirow{2}{*}{ User Accuracy (\%) } \\
\hline & & water & FYI & meteoric & MYI & & \\
\hline \multirow{4}{*}{ 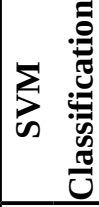 } & water & 0 & 0 & 0 & 0 & 0 & 0 \\
\hline & FYI & 0 & 29 & 1 & 0 & 30 & 96 \\
\hline & meteoric & 0 & 0 & 0 & 0 & 0 & 0 \\
\hline & MYI & 0 & 1 & 4 & 0 & 5 & 0 \\
\hline \multicolumn{2}{|c|}{ Total Segments } & 0 & 30 & 5 & 0 & 35 & \\
\hline \multicolumn{2}{|c|}{ Producer Accuracy (\%) } & 0 & 96.6 & 0 & 0 & & \\
\hline
\end{tabular}

Table 4.12 contains the overall accuracy assessment of the combined nine images. There is some discrepancy in the number of segments used in the accuracy assessment between various images as some had more or fewer segments due to coverage differences between FQ and FQW. Furthermore, ice islands were manually sampled with higher or lower sample numbers depending on the number of segments overlapping the ice islands. Overall, it was found that the primary classes of water, FYI and MYI could be classified rather confidently in many cases. Meteoric ice was not classified at all but was always misclassified as either FYI or MYI. Water and FYI detection was fairly strong although MYI detection was marginal.

Table 4.12: Overall accuracy assessment for the nine test images. Kappa was 0.38.

\begin{tabular}{|c|c|c|c|c|c|c|c|}
\hline & \multicolumn{4}{|c|}{ Reference Data } & \multirow{2}{*}{ Total Segments } & \multirow{2}{*}{ User Accuracy (\%) } \\
\hline & & water & FYI & meteoric & MYI & & \\
\hline \multirow{4}{*}{ 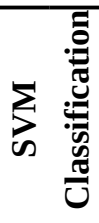 } & water & 35 & 1 & 0 & 0 & 36 & 97.2 \\
\hline & FYI & 6 & 138 & 48 & 1 & 193 & 71.5 \\
\hline & meteoric & 0 & 0 & 0 & 4 & 4 & 0 \\
\hline & MYI & 2 & 35 & 15 & 20 & 72 & 27.7 \\
\hline \multicolumn{2}{|c|}{ Total Segments } & 43 & 174 & 63 & 25 & 305 & \\
\hline \multicolumn{2}{|c|}{ Producer Accuracy (\%) } & 81.3 & 79.3 & 0 & 80 & & \\
\hline
\end{tabular}




\section{DISCUSSION}

\subsection{Geophysical Basis for Ocean Cover Separability}

Many of the THSD/G-H or RDA selected SAR variables were expected to perform well based upon literature for the discrimination of ocean covers. However, many more of them were not expected and consequently, the geophysical reasons for the usefulness of such SAR variables are not evident. The reasons given here for the unexpected SAR variables are largely speculative and require further research to substantiate, which the following discussion may serve to inspire.

\subsubsection{Backscatter}

Water had the lowest backscatter across nearly all polarizations, except VV where FYI had lower backscatter. Meteoric ice had the highest backscatter across all polarizations. This was expected due to salinity and textural differences between the ice covers and water.

Of all the polarizations, the HV polarization was found to explain the most variability in ocean cover identity as determined by RDA. The HV polarization was collinear to the gradient of ice type. The primary backscatter variables of A12, I12 and T12_real were found in the RDA to maximize the separability of ice types and explain a significant amount of variability in ocean cover identity. A12 was also identified by the further THSD/G-H tests to aid in reducing confusion between FYI and meteoric ice. A12 and I12, which are the amplitude and intensity of the cross-polarized channel, were somewhat expected based upon the results of (Scheuchl et al., 2001a) where the cross-polarized 
channel was able to separate sea ice and water at lower $\theta$. Furthermore, the crosspolarized channel over water is not influenced by wind, which was shown in the low correlation of wind, water and cross-polarized backscatter in Figure 4.8 and Figure 4.9. However, the use of T12_real or similar SAR variables has not been found in the literature. Reflectivity_11ov22, which is the co-pol ratio, however, has been used quite extensively in the literature (eg., (Dierking and Pedersen, 2012; Scheuchl et al., 2001a) because of its ability to separate rough FYI and water as well as smooth, rough and highly deformed FYI (Arkett et al., 2007; Scheuchl et al., 2001a).

\subsubsection{Double-bounce}

Among the three main scattering mechanisms (single, double, volume), the doublebounce scattering mechanism (e.g., in the variable T22) was the highest in meteoric ice. T22 was selected as useful for classification in THSD/G-H but not RDA. Other SAR variables such as Krogager_Kh, I12 and A12 were highly correlated and selected by RDA for being useful in explaining variability in ocean covers (Figure 4.4). Possible surface characteristics that may account for the higher double-bounce scattering in meteoric ice include edge dihedral reflection and the ice structure and ridges. 


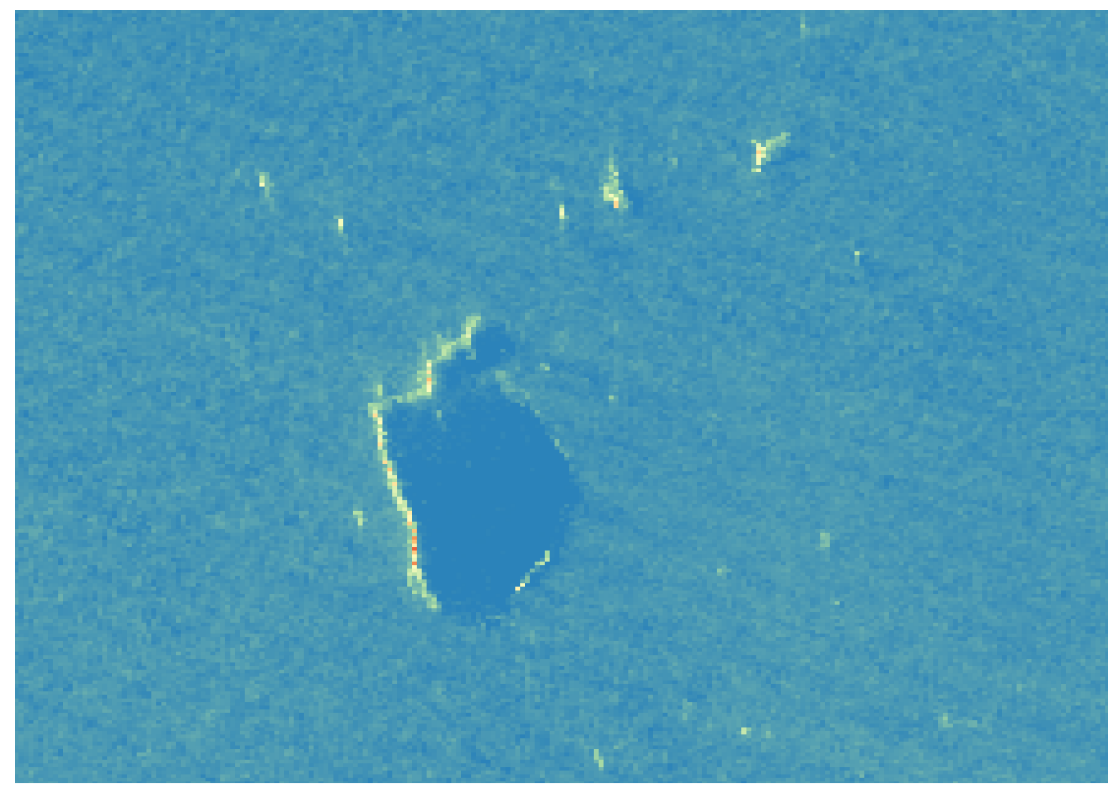

Figure 5.1: T22 double-bounce, with edge dihedral reflection towards RS2 from a Petermann Ice Island fragment in open water, symbology to highlight edges. Image acquired 2011/09/15-22:46:52. Blue is weaker double-bounce returns, yellow is medium, and red is strongest.

Edge reflection is quite evident within many SAR images (Figure 5.1) but seems to be dependent on whether there is a suitably high freeboard visible to the surrounding surface. Some ice islands embedded in sea ice floes may have rubble lining the edge which increases the double-bounce scattering (Jeffries and Sackinger, 1990). Other SAR images where the ice island is embedded in ice floes do not have the same degree of edge reflection. This rubble may diminish the double-bounce scattering attributable to the high freeboard since the rubble is more chaotic and may not reflect as distinctly to the side of the ice island. Furthermore, the edge that is visible to the incident SAR beam is the one that is detected while the opposite edges are in shadow. However, the median approach for the defining of a single value for each ocean cover does not account for this narrow edge as it is a small constituent part of the distribution of T22. The edges may have shifted the median value slightly higher but the median value is not the edges themselves. 
The summarizing approach of representing features as a single median value greatly reduced the computation complexity of the very large dataset. However, this was at a loss of description of the ocean cover variability in each image. Also, the manual segmentation of the ice island for training purposes may not have consistently included the edges. In fact, the rest of the ice island in this image has a lower double-bounce scattering than the rest of the open ocean and thus, edge effects are unlikely to explain the high T22 response.

For double-bounce scattering due to the ridges, it is unlikely the surface ridges are directly responsible for the high double-bounce of ice islands. This is because the surface ridges are not sufficient in amplitude nor wavelength to form a natural double bounce surface.

Another possibility is that internal reflections cause double-bounce due to randomly distributed scatterers (Atwood, 2013). However, this is also unlikely as there would need to be a suitable horizontally and vertically oriented scatterers for which to form the two reflections. A suitable vertical scatter could be the lake ice in the troughs. Typical ice islands are likely too thick to consider the ice/water interface to be a suitable horizontal surface since ice islands such as PII-A ranges in thickness of $50 \mathrm{~m}$ to $80 \mathrm{~m}$ (Halliday et al., 2012) and the penetration depth of freshwater ice, a close equivalent to low salinity ice of meteoric ice islands, is about $10 \mathrm{~m}$ at $5 \mathrm{GHz}$ (Shokr and Sinha, 2015).

To assess the location of the strongest double-bounce returns in relation to the surface ridges, which may lead to a better explanation, a T22 image of the Ward Hunt Ice Shelf was overlain on a Formosat-2 image (Figure 5.2). 


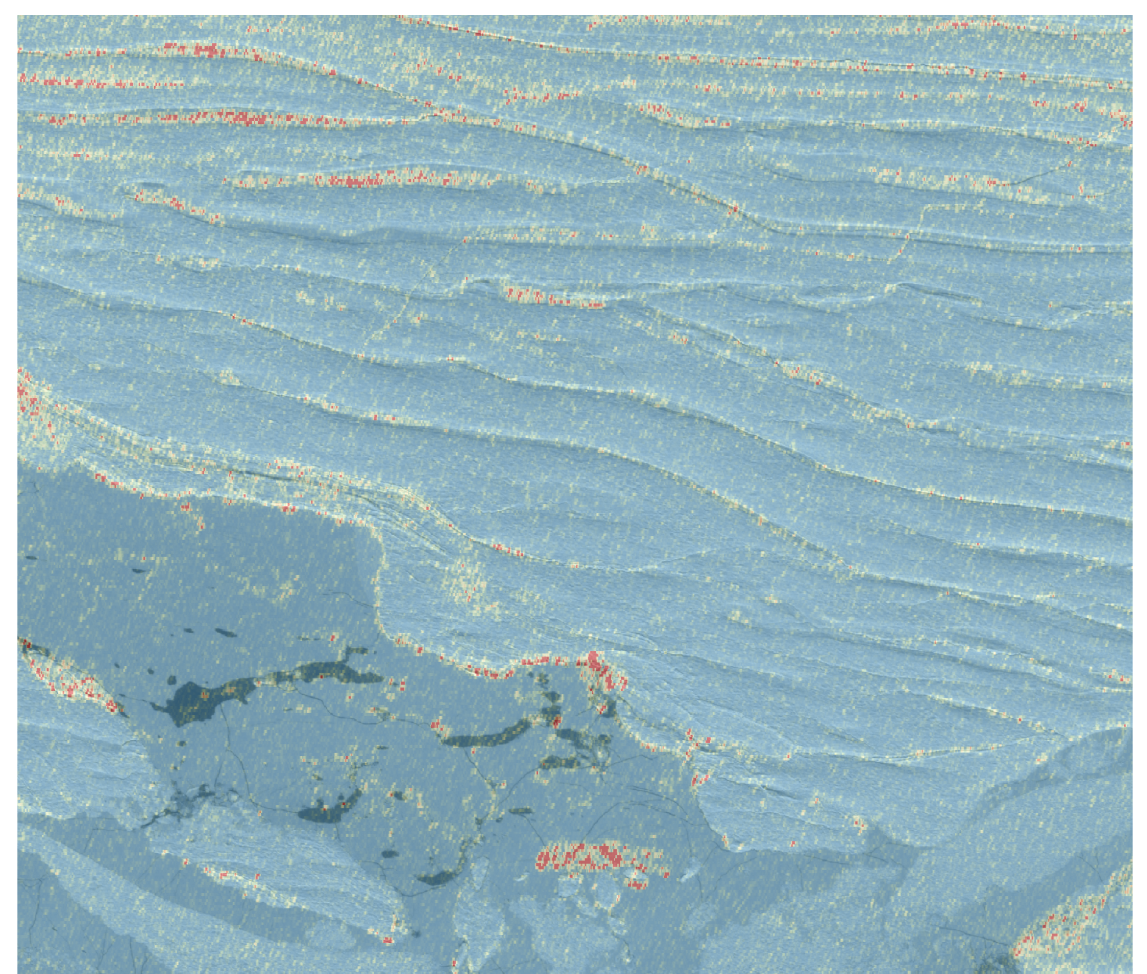

Figure 5.2: A T22 RS2 image overlain on a Formosat-2 image of the Ward Hunt Ice Shelf showing the pattern of double bounce scattering. Red represents strong T22 returns while yellow is medium and blue indicates weak T22 returns. The final co-registration was performed manually. RS2 image acquired 2009/03/0419:07:12. Formosat-2 image acquired 2008/08/28-17:10:27. Formosat-2 image courtesy of Planet Action 2008.

The narrow shape of the highest T22 returns suggests that the double-bounce is likely internal to the troughs. Furthermore, the T22 returns appear to be highest in the deeper, more shaded troughs. This suggests that the double-bounce returns are occurring from random coherent scatterers, possibly associated with the lake ice formed from seasonal melting and refreezing (Hattersley-Smith, 1957).

In addition to meteoric ice, MYI separability is highest using the double-bounce based scattering SAR variable Krogager_Kd which was identified by both THSD/G-H and RDA. The Krogager decomposition is for coherent surfaces (Krogager, 1990), so its selection by both THSD/G-H and RDA for sea ice is surprising. From the boxplots of 
Figure 4.3, a strong degree of overlap is present between MYI and meteoric ice. It is suspected that the double-bounce scattering is elevated due to rubble fields between highly modified MYI floes. Figure 5.3 demonstrates the differing Krogager_Kd values between the differing ocean covers of MYI, FYI, marine and meteoric ice as well as possibly open water. Although this image appears ideal for classification, there are many more of the training and testing images which are less than ideal and have greater confusion between the ocean covers. The brightest tones are either meteoric ice or rubble fields between MYI floes. FYI had the lowest Krogager_Kd response while the middle tones were due to MYI floes or marine ice. 


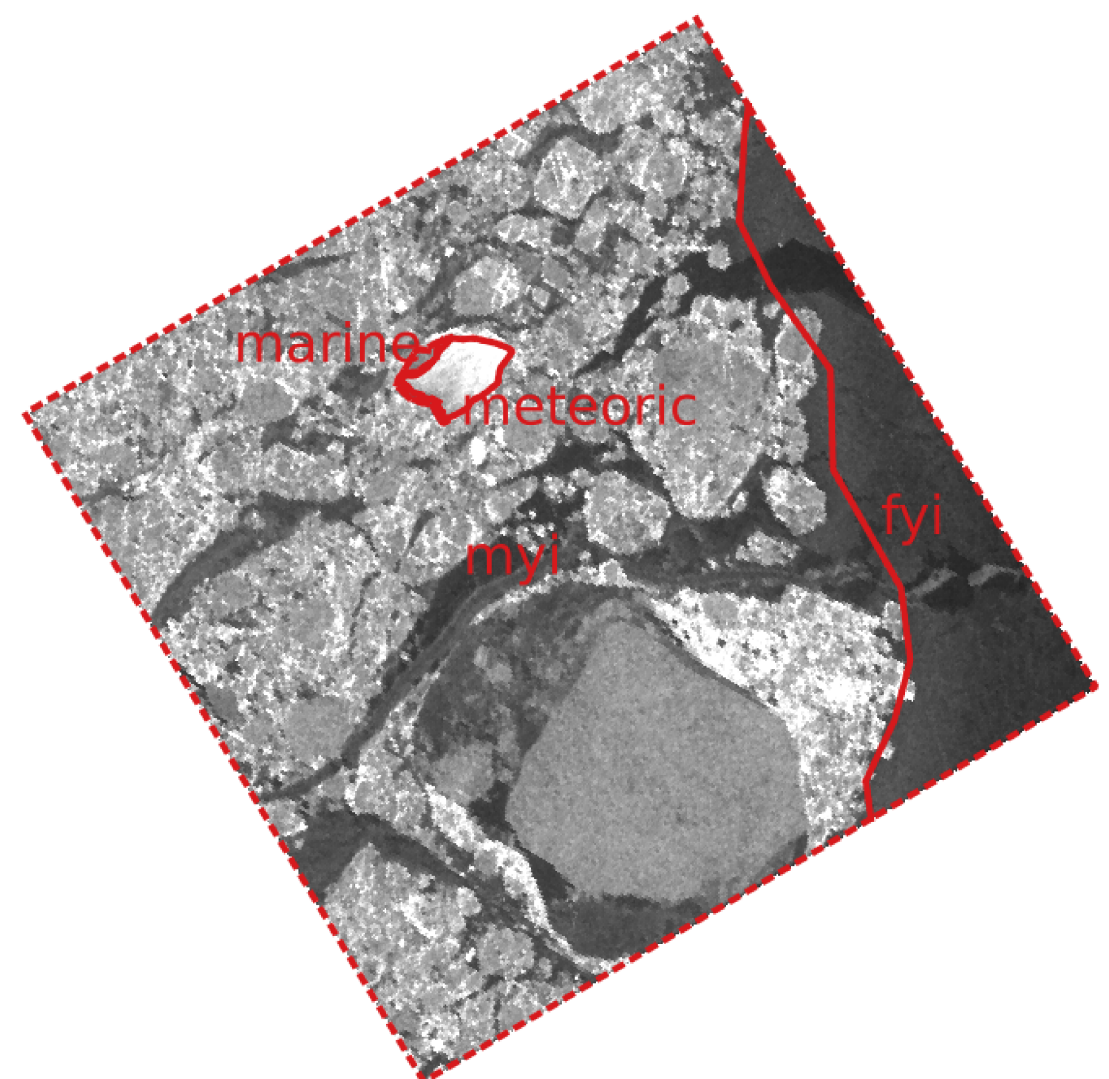

Figure 5.3: RS2 scene captured 2010/10/27-14:51:42 and converted to Krogager_Kd. This scene contains MYI, FYI, marine and meteoric and perhaps some small open water leads in the darkest tones. The rubble fields between the MYI floes have the highest Krogager_Kd, while the MYI floes have medium returns slightly less than marine ice. FYI has very low Krogager_Kd returns due to the likely near perfect specular reflection.

The SAR variable Krogager_Kh was selected by RDA and explained approximately $10 \%$ of ocean cover variability. Krogager_Kh is the helical component of the Krogager decomposition and is not quite a double-bounce variable like T22 or Krogager_Kd. However, helical scattering is commonly created by two or more dihedrals facing each other (Alberga et al., 2004; Lee and Pottier, 2009) and is strongly correlated to Krogager_Kd and T22 from THSD/G-H (Figure 4.4). Based upon the interpretation of T22 scattering in the troughs of meteoric ice, it may be surmised that Krogager_Kh forms in the deepest troughs or between floes when two dihedrals are facing each other. 


\subsubsection{Beta-Angle}

Beta-angle represents the target orientation around the radar line-of-sight (Mansouri et al., 2010) and has not been used in the literature to classify sea ice. It is not clear why Beta-angle was the most useful for FYI and Beta2 was the most useful for marine ice (Figure 5.1) as selected by THSD/G-H. Beta3 was also identified in the RDA forwardselection and is strongly correlated to Beta2 (Figure 4.4). From the knowledge of what the Beta-angle represents, it is theorized that the difference arises from the fact that FYI and marine ice are differently oriented from the other ocean covers. It is possible that FYI floes are more consistently flat since they have not experienced summer melting which alters the top surface of MYI (Figure 5.4). The Beta2 SAR variable maximizes marine ice separability. However, in a manner similar to Figure 5.4, the image is also noisy. This noisiness is not accounted for by the median value approach which uses one value to describe the distribution of a feature in a SAR variable and as such, the use of Beta and Beta2 in the training of the SVM classifier likely led to higher confusion when the training segments and classification SLIC segments were not analogous. 


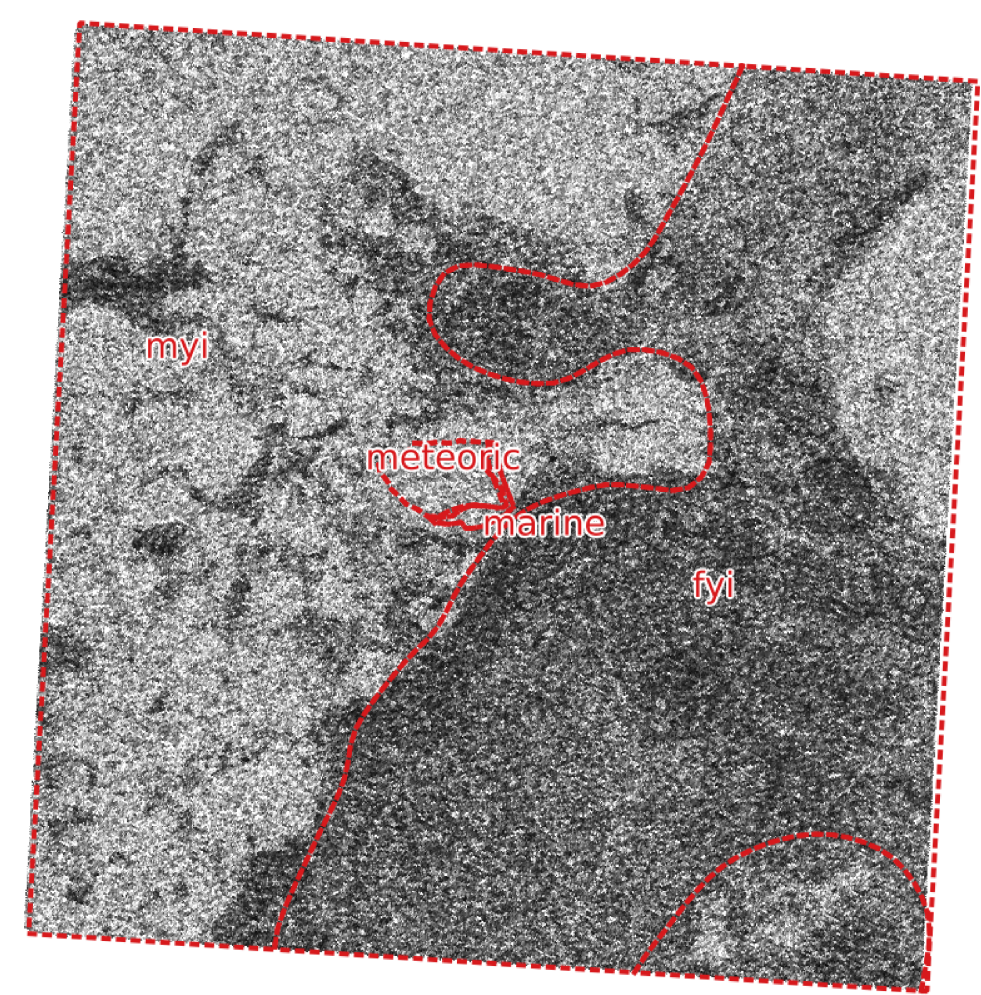

Figure 5.4: RS2 image captured on 2011/03/16-23:25:13 and converted to the Beta SAR variable. The MYI, meteoric and marine ice have similar Beta signatures while FYI has a lower signature.

\subsubsection{Other SAR Variables}

Neumann_tau was identified as a promising SAR variable in both THSD/G-H and RDA. The boxplots of Neumann_tau suggests that water has a higher particle orientation homogeneity than all ice types. However, water has a non-zero particle orientation randomness (Figure 4.3), which suggests that the particles are not completely linear. The interaction of SAR electromagnetic radiation with the ice surface depends upon salinity and ice structure in the form of the grain shape, orientation and inclusion characteristics (Richer-McCallum, 2015). Ice crystals, however, can be oriented in multiple directions (Berg, 2014). Depending on the age and type of ice, the orientation of these crystallographic or c-axes can vary (Berg, 2014). During initial freezing of sea ice, the ice 
platelets do not take a particular orientation (Mishchenko et al., 2000). As a consequence, pure ice, which forms a component of sea ice along with brine pockets, can have numerous different c-axes orientations while other ice types also have more homogenous c-axes orientations (Berg, 2014). MYI can also have an arbitrary orientation of its crystals. FYI in the uppermost layers (few centimetres) has a randomly oriented c-axes which transitions to horizontally oriented in the columnar structure of the ice (DeFranco and Dempsey, 1995).

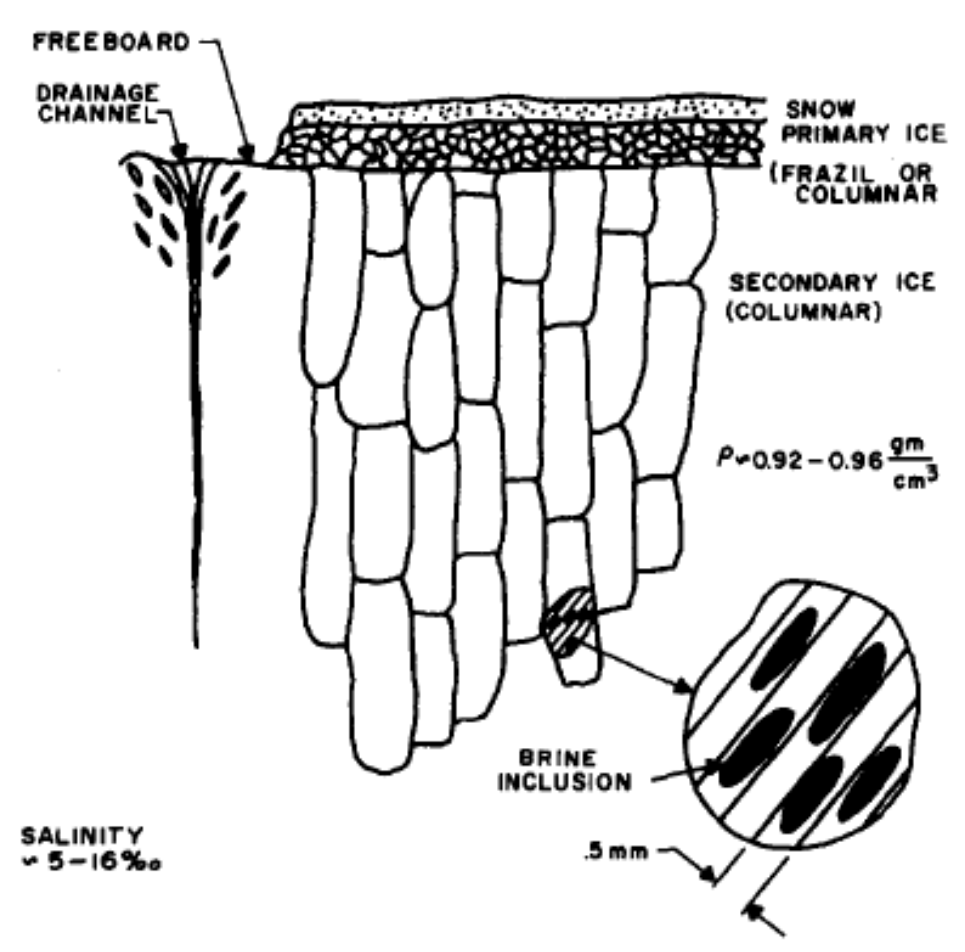

Figure 5.5: Geometry of FYI (Vant et al., 1978). The randomly oriented caxes in the upper portions of FYI transition to more horizontally oriented ice crystals in the columnar portion.

It is hypothesized that particles suspended within the water are likely more aligned, driven by currents and wave action at the surface. In Figure 5.6, there appears to be a contrast between the lower randomness of water to the higher particle orientation 
randomness of a meteoric ice island. Water is represented by the grey tones through the middle of the image, with darker tones of unknown origin near the bottom. These darker tones were not classified in the CIS charts and a Landsat-7 image acquired the same day did not show any features of note. It is possible that the darker tones are due to wind or current effects causing turbulence in the top surface and leading to particle orientation randomness.

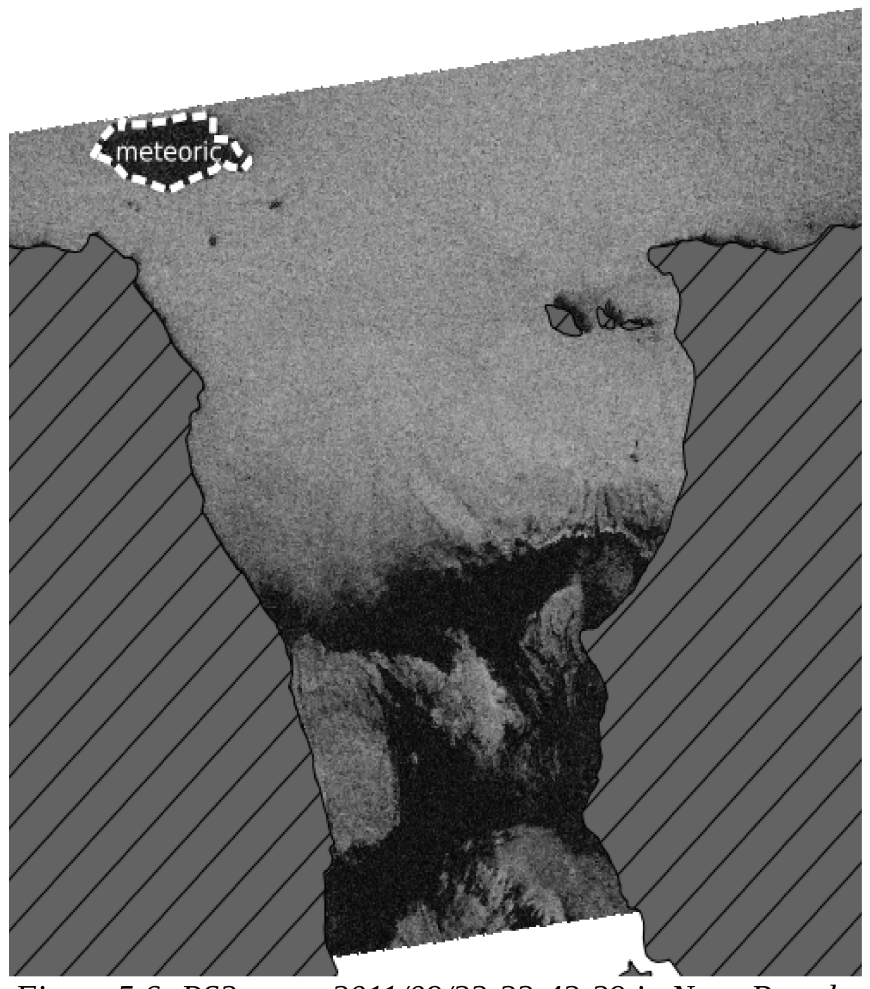

Figure 5.6: RS2 scene 2011/09/22-22:42:29 in Navy Board Inlet just west of Bylot Island. The lighter tones are lower Neumann_tau values corresponding to more consistent alignment of particles. The darker tones correspond to more random particle orientation. The ice island is to the upperleft, and water is the grey tones through the middle of the image. The darker tones to the bottom are unknown in origin.

Other SAR variables selected by the RDA forward-selection include 
TSVM_alpha_s2 and TSVM_phi_s3 which are from the Touzi decomposition. These decomposition variables have been mostly applied in wetland classification (Touzi, 2007). There is no literature that could be found on their use with sea ice and reasons for their discriminatory power in this study are not clear. Entropy_shannon and entropy_shannon_P_norm as selected by the RDA are the deviation from a Gaussian distribution in the SAR response and the normalized polarization component thereof (Chen et al., 2014; Eltoft et al., 2012; Réfrégier and Morio, 2006). Shannon entropy can also be described as the sum of intensity, degrees of polarization and coherence to form a measure of randomness (Lewis and Necsoiu, 2011; Morio et al., 2009). It differs from the more common 'classical' entropy in that classical entropy measures the importance of a single scattering mechanism to the others (Richards, 2009; van Zyl and Kim, 2011). Although it has been used in sea ice literature (Escobar et al., 2015), its practical meaning in terms of scattering principles is not completely understood.

\subsection{SAR Variable Selection and Multivariate Gradients using RDA}

ANOVA/THSD/G-H and RDA were the statistical tools used for the selection of SAR variables. Although the tools were used to answer similar questions for the selection of SAR variables, the methods selected different variables and for different reasons. However, as seen in Figure 4.4, many of the variables were highly correlated to one another. Furthermore, RDA was able to explore SAR variability in a complex multivariate space. 


\subsubsection{Direct Gradient Analysis}

The use of RDA in remote sensing is relatively new. Some researchers have used RDA to reduce complex multivariate datasets in mostly landcover or forestry applications in optical imagery (de la Cueva, 2008; Lobo et al., 2004; Pasher and King, 2010; Torontow and King, 2011). The use of RDA with SAR is a novel approach. RDA was found in this study to be an excellent way to explore and visualize relationships between variables and groups of variables. RDA was also used to select variables which explain the most variation in the desired classes. This method can be helpful in guiding the creation of future classifiers.

Overall, RDA explained a great amount of variability in most cases; for example, all axes for the first five forward-selected SAR variables explained $47 \%$, however, the significance of this is difficult to compare to other studies as RDA has not been used with SAR before. In comparison, Pasher and King (2010) used RDA axis one with five image spectral and spatial predictor variables to explain 35\% of the variance in a set of forest structure and DEM variables and Torontow and King (2011) used a six predictor model to explain $32 \%$ of the variance in a similar set of structure, composition and DEM data using RDA axis one and two. However, both these investigations used RDA to predict multivariate forest attributes and not binary class identity as in this study.

Based upon the triplot of Figure 4.9, overall, ocean cover identity was found to be more important than the confounding variables. In theory, ocean cover classification should be possible where the confounding variables are less important than the ocean cover identity. Ocean covers which are strongly correlated to confounding factors are 
likely to be difficult to distinguish.

For RDA Question 1 and the forward-selection of SAR variables which best explain variability in the ocean cover identity, it was found that there was a decrease in the significance after the $21^{\text {st }}$ SAR variable. It appears that at the 0.05 significance level, the limit of explained variability in the ocean cover identity is approximately $73 \%$. Notably, there were less SAR variables beyond the $21^{\text {st }}$ which were significant. Furthermore, the selection of A12, I12 cross-polarized amplitude and intensity matches with the results of (De Abreu et al., 2011) where it was found that the cross-polarized channels were the best polarization for separability.

For the RDA Question 2, assessing the relationship between the confounding factors and the the SAR variables, it was found that water behaved differently in general to the confounding factors. Water was associated with the gradients of $\theta$ and wind speed, as anticipated. Water was also aligned with temperature, likely because higher temperature leads to more open water while lower temperature leads to less open water and the lowest temperatures should have little to no open water. Reflectivity_11ov22 was found to strongly fall along a gradient of temperature, which may be due to the effects of salinity as alluded to by Dierking and Pedersen (2012).

In Question 3 where all the confounding factors and ocean cover identity are used to explain the SAR variability, the water remains quite distant from the ice covers. The ice covers seem to form a gradient of salinity along from FYI, marine, MYI and meteoric ice. From this triplot, it is was noticed that A12, I12, entropy_shannon, Krogager_Kh and Krogager_Kd are likely helpful at detecting salinity differences as well as, to a lesser 
degree, some $\theta$ differences as they are aligned to the gradient of FYI, marine, MYI and meteoric ice. Furthermore, many confounding variables such as wind speed and temperature are more important to the RDA model than ocean cover identity, suggesting that wind speed and temperature may lead to a high degree of confusion between ocean covers. Temperature, for example, is related to seasonal inversions of backscatter in sea ice (Yackel et al., 2007). $\theta$ also accounts for some confusion between the ocean covers as it closely follows the ice cover gradients, however this effect is far less than temperature. As expected, wind speed is not associated with ocean covers as their should be unaffected by differing wind speeds.

In Question 4 where the effects of the identity of ice islands was accounted for from Question 3, pseudo-replication was found to account for approximately 5\% of the variance of the SAR variables, which was not a substantial proportion of the total explained variability as compared with other groups of variables. Based on this analysis, it seems the impact of breaking the assumption of independence by re-imaging the same ice islands is not a serious issue. However, another type of pseudo-replication may exist, given the fact that each offspring ice island comes from a parent ice shelf and has similar geophysical characteristics. The effects of pseudo-replication of observations through sibling/offspring relationships was not determined here.

The time lag between the CIS regional chart and the RS2 image acquisition was considered to be an issue with the method used and the implications were addressed with the variable 'offset' in RDA Question 2 and 3. It was found that the offset was not a primary cause of variability but it was visible in the RDA analysis. In RDA the offset was 
either the shortest or second shortest gradient to $\Phi$. Overall, the effect of this offset was minimal but detectable.

\subsection{Comparison of ANOVA/THSD/G-H and RDA Selection Approaches}

The THSD/G-H and RDA approaches to the selection of SAR variables often selected SAR variables which although were not exactly the same, were either similar or correlated well. The SAR variables selected by the RDA forward-selection approach may be more suitable for use in classification than those chosen by the THSD/G-H approach. This is due to RDA being an approach which inherently accounts for multivariate dimensionality when selecting the SAR variables, as opposed to univariate selection using THSD/G-H. However, the SAR variables chosen with RDA were more complex to interpret since they are chosen based on how well they explain ocean covers over the entire range of variability, not on a difference of means. With ANOVA, the SAR variables found to be the most useful matched well with those of high explanatory power in the RDA forward-selection (Appendix C and Section 4.4.2). The THSD/G-H method also identified many similar SAR variables to the RDA forward-selection approach such as Krogager_Kd, Neumann_tau and the beta angle.

It was found in the quantitative accuracy assessment that much of the confusion was between meteoric ice and FYI, meteoric ice and MYI, and FYI and MYI. For these pairwise confusions, additional SAR variables were selected in an effort to reduce these errors. These variables are A12, VanZyl3_Dbl and TSVM_alpha_s3 to assist with the confusions of meteoric/FYI, meteoric/MYI and FYI/MYI. The p-values were all 
$<0.00001$ for these selected variables. TSVM_alpha_s3 here is the third eigenvector scattering (Touzi, 2007). However, the selected SAR variables do not account for colinearities. These complementary SAR variables also matched up well with RDA selected variables which indicates they are associated with unexplained variance. Overall, both THSD/G-H and RDA approaches selected previously unexplored SAR variables instead of more commonly used ones which underscores the need for the exploratory statistical approaches used in this thesis as a complement to more traditional remote sensing approaches. For instance, coherent decomposition variables like Krogager_Kd would not have been identified useful for separability with other approaches.

\subsection{SLIC Segmentation and SVM Classification}

The SLIC segmentation approach generally worked well in spite of the inherent 'noisiness' of SAR. However, the tuning of SLIC parameters to optimize the segmentation

process was difficult. The number of clusters, sizes, smoothing, number of k-means iterations and compactness were tuned to balance adherence to boundaries, over- and under- segmentation as well as shape simplicity across most images. Simpler segments were preferred as they would match with ice floe shapes. However, no set of parameters was suitable for all images. This compromise led to segmentation which worked well for images with heavy ridging and distinct floes, but images which did not have distinct floes and were heavily compacted ice did not segment as effectively. Furthermore, the SLIC segments in consolidated ice pack were very uniform and often were not on ice floe boundaries. A further post-segmentation aggregation may have been ideal to combine neighbouring segments which were similar. Tools such as multi-resolution segmentation 
in eCognition may have provided a superior segmentation. Multi-resolution segmentation using all classification SAR variables could have led to better boundary adherence and segments which more realistically varied in size.

In the SLIC, it was noticed that the segments were slightly warped. This is due to the segmentation working on unprojected ground range SAR images. Since the resolution is different in either direction, the SLIC favours segments in one direction as opposed to the other. This effect is further maximized as the compactness factor is increased, leading to segments that are more distance-proximity based than colour-proximity based, especially over low-colour-variability regions such as water (Figures 4.10, 4.11 and 4.12). It was decided to continue using the SLIC with unprojected SAR images as all statistical analysis was done using unprojected ground range images. It is possible to account for varying resolution in either direction within SLIC however this was not explored.

The SVM classification was able to train on the large and complex training dataset and predict the identity of the SLIC segments in the testing images to some degree of confidence. However, the between-class confusion was still high, especially for MYI/FYI, MYI/meteoric and FYI/meteoric. Also, no samples of meteoric ice were correctly classified. Some of this confusion could be due to a poor segmentation in SLIC which leads to ice islands separating into two or more segments, or segments containing more than just the ice island. Also, some of the confusion was also likely due to the THSD/G-H selected SAR variables not being selected to account for multivariate dimensionality (see discussion in Section 5.3). The SVM class confusion follows what was expected based upon the RDA triplot of Figure 4.9 where water could be confidently 
classified but there was significant confusion between the ice types. The ice confusion was likely even higher than the RDA triplots suggest, since the SAR testing images were acquired in the winter while the testing images were acquired during the summer where melting conditions would have been an issue (Appendix B).

The selection of SAR imagery for training was driven towards selecting as many images as possible for characterizing the variability of ice islands. However, there was a limited availability of FQ(W) RS2 imagery which contains ice islands, especially of both types. The selection of nine testing images post-training seemed to be a satisfactory number but it was discovered very late in the analysis that in an oversight, marine ice islands were not found in the testing imagery.

\subsection{Future Work}

\subsubsection{Use of RDA Forward-Selected SAR Variables in an SLIC/SVM Classifier}

The SAR variables used in the classification were selected using the THSD/G-H approach to aid interpretation. However, the next logical extension is to use the 12 SAR variables what were identified with RDA. Beyond that, no significant changes are likely required in the SLIC/SVM approach to achieve an improved classification accuracy. However, it may be useful to explore optimization of the parameters of $\mathrm{C}$ and $\Upsilon$ using the grid search approach to further improve the SVM classifier and tune SLIC for better adherence to ice island boundaries.

\subsubsection{Include Feature Formations to Describe Differences in Ice Forms}

In this study, the form of the ice was not included as a description of the ice cover. 
However, the CIS provides some description as to the form of the ice floe into categories such as pancake, brash, cake and differing floe sizes (MANICE, 2005). Conceivably, the form could be another confounding factor that confuses the classification identity as the form can significantly alter the backscatter (Johannessen et al., 2006). However, more SAR images would need to be used throughout the assessment to ensure there were enough samples to satisfy each measured category.

\subsubsection{Region Aggregation in SLIC}

One goal of the SLIC was to segment the regions to match as closely as possible to the areas used in training so that the determined median values would be similar, allowing for a more accurate classification. It was noticed that areas of water were well segmented and based on the desired approximate number of clusters. However, ice floes especially with substantial ridging were over-segmented. To counter this oversegmentation, a post-cluster aggregation approach may be useful. An adaptive colourspace approach could be developed to ignore the number of clusters parameter (which limited the number of clusters in the segmentation and was fixed for all classifications) and instead would determine whether adjacent clusters should be aggregated based upon whether the cluster means were significantly different. Such an approach could be implemented using G-H tests of paired neighbouring cluster regions and a fixed statistical significance. An adaptive significance could be integrated such that all adjacent pairwise G-H significances are computed. All pairwise clusters below the 0.05 significance value should be aggregated. This aggregation could continue until the minimum pairwise G-H is greater than a critical significance value, such as $\mathrm{p}<0.05$. With a robust segmentation 
approach, it would also be possible to add GIS-based geometric statistics to better detect large floes with specific dimensions which could be ice islands.

\subsubsection{Application of RDA Approach to other Classifications in SAR and Optical Imagery}

In other classification problems with a large number of unique and suitable remote sensing variables and a wide degree of variability, RDA is a highly useful approach to guide classification development. Forward-selection with RDA is a good way to select image variables which explain the most variability in identity variables. Furthermore, RDA provides a visual means of assessing dependent and independent variables in a complex multivariate space and greatly simplifies interpretation. Ice classification is influenced by many confounding factors which form complex relationships with discriminating variables based on SAR backscatter. Visual analysis of RDA triplots clarified the relationships between confounding factors, ocean cover identity, SAR variables and pseudo-replication.

This technique could be extended to similar classification problems such as oil spill identification and delineation in SAR where the confounding variables such as differing oil types, age of the oil slick, and wind speed may hinder classification. However, separation between look-alikes such as low wind areas and oil slicks is a notable challenge (Brekke and Solberg, 2005). RDA forward-selection would be a suitable approach to select a series of SAR variables for detecting oil slicks in a multivariate classification. Further SAR variables could be selected to identify common look-alikes. Combining these sets of SAR variables could lead to consistently accurate oil slick 
classification. The relationship between confounding factors, oil slick and lookalike identity, and SAR variables could also be explored with RDA triplots. 


\section{CONCLUSION}

With the hazard of ice islands in the Arctic waters, it is crucial that SAR detection methods are improved to enhance CIS' “early warning systems” that might be used by offshore operations. The goal of this study was to investigate the interaction between SAR, ice islands and confounding factors in order to improve ice island detection.

From 123 available polarimetric SAR variables, several were selected as being

promising at separating ice islands. Five SAR variables were selected with THSD/G-H tests which maximized separability between oceans covers. These tests identified Beta, Krogager_Kd, Neumann_tau, T22, Beta2 as the most useful SAR variables to separate FYI, MYI, water, meteoric and marine ice islands respectively. Also, forward-selection in RDA was performed to select a different group of 12 SAR variables which explained the greatest variability in ocean cover identity in Section . It was noticed that THSD/G-H selection of SAR variables were similar to those of RDA. Among the selected variables were those that were based on cross-polarization backscatter, co-polarization ratio, and some variables derived from decompositions like Neumann_tau and Krogager_Kh. These unexpected SAR variables selected by either THSD/G-H or RDA have largely not been used previously in ocean cover classification and warrant further study. With RDA, it was found that the optimal number of SAR variables was approximately 12 and these explained $65.5 \%$ of variability, higher than the five selected with THSD/G-H. Above 21 SAR variables, the explanatory power did not improve dramatically.

Another objective was to determine how much variability in the polarimetric SAR 
variables could be explained by SAR geometry and environmental conditions. Based on RDA, these confounding variables were found to explain $23 \%$ of the variability in SAR. Water, as expected, was found to behave differently from the ice-based ocean covers. Also, temperature explained the most variability of the confounding variables in the ocean covers, suggesting this is perhaps a large source of misclassification of sea ice. With the inclusion the ocean cover identity with the other confounding variables, $61.7 \%$ of the variability in SAR could be explained. The expected dependence of SAR variables on $\theta$ and temperature was also demonstrated. Further RDA analysis included the effects of ocean cover identity and compared these effects to those of the confounding factors. With the inclusion of ocean cover identity, a suspected salinity-based gradient of the ocean covers was identified. Finally, it was found that the pseudo-replication accounted explained little variability therefore, the observations were like not biased much by the lack of independence in the experimental design. Based on the RDA analysis, it is believed that sea ice classification could potentially be improved with the inclusion of confounding factors such as temperature, $\theta$, and wind speed.

The last research objective was to explore the usefulness of an unsupervised classification method using the best polarimetric SAR variables as identified with THSD/G-H to discriminate ice islands and other ice types. Moderate confusion was found in the SVM classification between water, FYI and MYI. However, meteoric ice islands were not identified and marine ice islands were not found in the testing imagery. Adding supplementary SAR variables to improve the classifier and three SAR variables were identified to aid in specific pairwise confusion. The SLIC/SVM approach led to 
reasonable MYI, FYI and water classifications despite the inability to classify ice islands. It is suspected that a different segmentation approach that better adhered to actual ocean cover boundaries could lead to a more accurate classification with SVM.

This thesis leveraged a large dataset of FQ(W) imagery and computational resources to generate 123 SAR variables. This impressive dataset was reduced using a statistical approach to select SAR variables as well as identify relationships between SAR variables and confounding factors. This big data approach can also be applied to other remote sensing problems such as oil slicks. The SAR variables explored here may also be useful in future sea ice classification. CIS classification uses the wide-swath ScanSAR imagery which lacks quad-polarimetric information. However, upcoming missions such as the RADARSAT Constellation Mission (RCM) will offer compact polarimetry, which provides a method of polarimetric information in enhanced swath widths. 


\section{REFERENCES}

Abdi, H., Williams, L.J. (2010). Tukey’s honestly significant difference (HSD) test, in: Salkind, N. (Ed.), Encyclopedia of Research Design. SAGE Publications Limited, Thousand Oaks, California, 1-5

Achanta, R., Shaji, A., Smith, K., Lucchi, A., Fua, P., Süsstrunk, S. (2012). SLIC superpixels compared to state-of-the-art superpixel methods. Pattern Analysis and Machine Intelligence, IEEE Transactions on, 34, 2274-2282. DOI 10.1109/TPAMI.2012.120

Achanta, R., Shaji, A., Smith, K., Lucchi, A., Fua, P., Süsstrunk, S. (2010). SLIC superpixels (EPFL Technical Report No. 149300)

Agashe, S. (2013). Polarimetric decomposition of SAR data for forest structure assessment (Master's thesis, Wireless, Photonic and Space Engineering). Department of Earth and Space Sciences, Chalmers University of Technology

Al-Azzawi, A. (2006). Photonics: Principles and Practices. Taylor \& Francis, Boca Raton, Florida, 968

Alberga, V., Krogager, E., Chandra, M., Wanielik, G. (2004). Potential of coherent decompositions in SAR polarimetry and interferometry, in: International Geoscience and Remote Sensing Symposium. 1792-1795. DOI 10.1109/IGARSS.2004.1370682

Aoki, S. (2009). Games-Howell R function. Gunma University, Japan. http://aoki2.si.gunma-u.ac.jp/R/src/tukey.R

Arkett, M., Flett, D., De Abreu, R. (2007). C-Band multiple polarization SAR for ice monitoring - what can it do for the Canadian Ice Service. Presented at the Envisat Symposium

De'ath, G. (2002). Multivariate regression trees: a new technique for modeling speciesenvironment relationships. Ecology, 83, 1105-1117. DOI 10.1890/00129658(2002)083[1105:MRTANT]2.0.CO;2

Atwood, D. (2013). Wave propagation model for coherent scattering from a randomly distributed target. Presented at the PollnSAR Workshop, Frascati, Italy

Bahlmann, C., Haasdonk, B., Burkhardt, H. (2002). On-line handwriting recognition with support vector machines - a kernel approach. Presented at the Eighth International Workshop on Frontiers in Handwriting Recognition (IWFHR'02), IEEE Computer Society, 49-54

Ben-Hur, A., Weston, J. (2010). A user's guide to support vector machines, in: Carugo, O., Eisenhaber, F. (Eds.), Data Mining Techniques for the Life Sciences, Methods in Molecular Biology. Humana Press, 223-239. DOI 10.1007/978-1-60327-2414_13 
Benjamini, Y., Hochberg, Y. (1995). Controlling the false discovery rate: a practical and powerful approach to multiple testing. Journal of the Royal Statistical Society. Series B (Methodological), 57, 289-300. DOI 10.2307/2346101

Berg, A. (2014). Spaceborne synthetic aperture radar for sea ice observations, concentration and dynamics (PhD, Department of Earth and Space Sciences). Chalmers University of Technology, Gothenburg, Sweden, 72

Betbeder, J., Nabucet, J., Pottier, E., Baudry, J., Corgne, S., Hubert-Moy, L. (2014). Detection and characterization of hedgerows using TerraSAR-X imagery. Remote Sensing, 6, 3752-3769

Bindoff, N.L., Stott, P.A., AchutaRao, M., Allen, M.R., Gillett, N., Gutzler, D., Hansingo, K., Hegerl, G., Hu, Y., Jain, S. (2013). Detection and attribution of climate change: from global to regional, in: Climate Change 2013 - The Physical Science Basis. Cambridge University Press, Cambridge, 867-952. DOI 10.1017/CBO9781107415324.022

Boost-Technologies. (2006). Wave measurements from space using ASAR image and wide swath mode. http://www.boost-technologies.com/esa/sar_wave.html

Bouvet, M. (2010). Getasse30 elevation model. http://seadas.gsfc.nasa.gov/help/visat/GETASSE30ElevationModel.html

ter Braak, C.J. (1990). Update notes: CANOCO version 3.10. Agricultural Mathematics Group, Wageningen, 35

ter Braak, C.J, Smilauer, P. (2004). Canoco. Microcomputer Power, Ithaca, New York

Brekke, C., Solberg, A.H.S. (2005). Oil spill detection by satellite remote sensing. Remote Sensing of Environment, 95, 1-13. DOI 10.1016/j.rse.2004.11.015

Campbell, J.B., Wynne, R.H. (2011). Introduction to Remote Sensing. Guilford Press, New York, 667

Canadian Ice Service (2005). MANICE: manual of standard procedures for observing and reporting ice conditions. Environment Canada, Meteorological Service of Canada, Downsview, Ontario, 146. http://www.ec.gc.ca/glaces-ice/default.asp? lang $=\mathrm{EN} \& \mathrm{n}=4 \mathrm{FF} 82 \mathrm{CBD}-1$

Canadian Ice Service. (2015). Canadian Ice Service. http://www.ec.gc.ca/glaces-ice/

Chang, C.-C., Lin, C.-J. (2011). LibSVM: A library for support vector machines. ACM Transactions on Intelligent Systems and Technology. Software available at http://www.csie.ntu.edu.tw/ cjlin/libsvm., 2, 27:1-27:27

Chan, Y.K., Koo, V.C. (2008). An introduction to synthetic aperture radar (SAR). Progress In Electromagnetics Research B, 2, 27-60

Chen, C.H. (2007). Image Processing for Remote Sensing. CRC Press, Boca Raton, Florida, 400.

Chen, Y., He, X., Wang, J., Xiao, R. (2014). The influence of polarimetric parameters and 
an object-based approach on land cover classification in coastal wetlands. Remote Sensing, 6, 12575. DOI 10.3390/rs61212575

Cloude, S.R., Pottier, E., Boerner, W.M. (2002). Unsupervised image classification using the entropy/alpha/anisotropy method in radar polarimetry, in: 2002 JPL-AIRSAR Earth Science Applications Workshop. Pasadena, United States, 27-60

Conyers, L.B. (2013). Ground-penetrating radar for archaeology, 3rd ed, Geophysical methods for archaeology. AltaMira Press, Lanham, 241

Copland, L., Mueller, D.R., Weir, L. (2007). Rapid loss of the Ayles Ice Shelf, Ellesmere Island, Canada. Geophysical Research Letters, 34, L21501. DOI 10.1029/2007GL031809

Cotell, I.M. (1960). Activities on Ice Island T-3, 1959-60. Polar Record, 10, 385-386

Crawford, A. (2013). Ice island deterioration in the Canadian Arctic: Rates, patterns and model evaluation (M.Sc., Department of Geography and Environmental Studies). Carleton University, Ottawa, ON, 140

De Abreu, R., Arkett, M., Cheng, A., Zagon, T., Vachon, P.W., Wolfe, J., Mueller, D.R. (2011). RADARSAT-2 mode selection for maritime surveillance (External Client Report). Defence Research and Development Canada, Ottawa, 185

DeFranco, S.J., Dempsey, J.P. (1995). The fracture of sea ice, in: Erdogan, F. (Ed.), Fracture Mechanics. ASTM International, Philadelphia, 433-445. DOI 10.1520/STP1220-EB

de la Cueva, A.V. (2008). Structural attributes of three forest types in central Spain and Landsat ETM+ information evaluated with redundancy analysis. International Journal of Remote Sensing, 29, 5657-5676. DOI 10.1080/01431160801891853

Deng, L., Wang, C. (2014). Improved building extraction with integrated decomposition of time-frequency and entropy-alpha using polarimetric SAR data. IEEE Journal of Selected Topics in Applied Earth Observations and Remote Sensing, 7, 4058-4068. DOI 10.1109/JSTARS.2013.2281594

Devadas, R., Denham, R.J., Pringle, M. (2012). Support vector machine classification of object-based data for crop mapping, using multi-temporal LANDSAT imagery. International Archives of the Photogrammetry, Remote Sensing and Spatial Information Sciences, XXXIX-B7, 185-190

De Veaux, R.D., Velleman, P.F., Bock, D.E. (2012). Stats: Data and Models, 3rd ed. Pearson Education, Boston, Massachusetts, 973.

DeVona, J. (2013). Ground-Track-Generator. https://github.com/anoved/Ground-TrackGenerator

Dierking, W., Garrity, C., Ramsier, R.O. (1994). Interpretation of radar signature observed in SAR images of ice island T-3 by means of backscatter modelling. EARSeL Advances in Remote Sensing, 3, 31-43

Dierking, W., Pedersen, L.T. (2012). Monitoring sea ice using Envisat ASAR - A new era 
starting 10 years ago. Geoscience and Remote Sensing Symposium (IGARSS), 2012 IEEE International, 1852-1855. DOI 10.1109/IGARSS.2012.6351147

Doerry, A.W., Dickey, F.M. (2004). Synthetic aperture radar. Optics \& Photonics News, 15, 28-33. DOI 10.1364/OPN.15.11.000028

Eltoft, T., Doulgeris, A., Anfinsen, S.N. (2012). Analysis of textured PolSAR data by Shannon entropy. Geoscience and Remote Sensing Symposium (IGARSS), 2012 IEEE International, 1449-1452. DOI 10.1109/IGARSS.2012.6351262

Environment Canada. (2013). Petermann Glacier Ice Island 2012. http://www.ec.gc.ca/default.asp?lang=En\&n=592AB94B-1\&news=63B51150B0B0-438F-94CA-A1CDDFA86A25

Esch, T., Schenk, A., Thiel, M., Ullmann, T., Schmidt, M., Dech, S. (2010). Land cover classification based on single-polarized VHR SAR images using texture information derived via speckle analysis. Geoscience and Remote Sensing Symposium (IGARSS), 2010 IEEE International, 1875-1878. DOI 10.1109/IGARSS.2010.5650031

Escobar, I., Cardenas, C., Lopez, C., Johnson, E. (2015). Polarimetric decomposition and Shannon Entropy in sea ice. Presented at the 15th Meeting of the International Ice Charting Working Group, Punta Arenas, Chile

European Space Agency. (2014a). PolSARpro. https://earth.esa.int/web/polsarpro/home

European Space Agency. (2014b). Next ESA SAR Toolbox. https://earth.esa.int/web/nest/home

European Space Agency. (2014c). Scientific Background. https://earth.esa.int/handbooks/asar/CNTR1-1-2.html

European Space Agency. (2013). Help - Calibration. http://corp.array.ca/nestweb/help/operators/CalibrationOp.html

Falkner, K.K., Melling, H., Münchow, A.M., Box, J.E., Wohlleben, T., Johnson, H.L., Gudmandsen, P., Samelson, R., Copland, L., Steffen, K., Rignot, E., Higgins, A.K. (2011). Context for the Recent Massive Petermann Glacier Calving Event. EOS, Transactions American Geophysical Union, 92, 117-118. DOI 10.1029/2011EO140001

Fisheries and Oceans Canada. (2010). Key findings from International Polar Year 20072008 at Fisheries and Oceans Canada: executive summary. Fisheries and Oceans Canada, Communications Branch, Ottawa

Fissel, D., Mudge, T., Chave, R., Stone, M., Kanwar, A., Bard, A., Buermans, J. (2011). Real-time pack ice monitoring systems - identification of hazardous sea ice using upward looking sonars for tactical support of offshore oil and gas projects.

Presented at the Arctic Technology Conference, Offshore Technology Conference, Houston, Texas, USA, 1-9

Flett, D., De Abreu, R., Arkett, M., Gauthier, M.-F. (2008). Initial Evaluation of Radarsat- 
2 for Operational Sea Ice Monitoring. Presented at the International Geoscience and Remote Sensing Symposium, IEEE, Boston, Massachusetts, I-9 - I-12.

Freeman, A., Durden, S.L. (1998). A three-component scattering model for polarimetric SAR data. IEEE Transactions on Geoscience and Remote Sensing, 36, 963-973. DOI 10.1109/36.673687

Fuglem, M., Jordaan, I. (In Press.). Risk analysis and hazards of ice islands, in: Copland, L., Mueller, D.R. (Eds.), Arctic Ice Shelves and Ice Islands. Springer SBM, Dordrecht

Gagnon, L., Jouan, A. (1997). Speckle filtering of SAR images: a comparative study between complex-wavelet-based and standard filters, in: SPIE Proceedings. 80-91. DOI 10.1117/12.279681

Games, P.A., Keselman, H.J., Clinch, J.J. (1979). Tests for homogeneity of variance in factorial designs. Psychological Bulletin, 86, 978-984. DOI 10.1037/00332909.86.5.978

Gardener, M. (2014). Community Ecology: Analytical Methods Using R and Excel, 1st ed. Pelagic Publishing, Exeter, UK, 556

GDAL Development Team. (2014). GDAL - Geospatial Data Abstraction Library. Open Source Geospatial Foundation. http://www.gdal.org

Gill, J.P., Yackel, J.J. (2012). Evaluation of C-band SAR polarimetric parameters for discrimination of first-year sea ice types. Canadian Journal of Remote Sensing, 38, 306-323

Government of Canada. (2015). GDPS data in GRIB2 format: 25 km. http://weather.gc.ca/grib/grib2_glb_25km_e.html

Guillaso, S., Schmid, T., López-Martínez, J., D’Hondt, O. (2015). Application of a new polarimetric filter to Radarsat-2 data of Deception Island (Antarctic Peninsula region) for surface cover characterization. ISPRS - International Archives of the Photogrammetry, Remote Sensing and Spatial Information Sciences, XL-7/W3, 1035-1039. DOI 10.5194/isprsarchives-XL-7-W3-1035-2015

Halliday, E., King, T., Bobby, P., Copland, L., Mueller, D. (2012). Petermann Ice Island "A" survey results, offshore Labrador. Presented at the OTC Arctic Technology Conference, Offshore Technology Conference, Houston, Texas, USA, 1-14. DOI 10.4043/23714-MS

Hattersley-Smith, G.F. (1962). The term “ice island.” Journal of Glaciology, 4, 131 Hattersley-Smith, G.F. (1957). The rolls on the Ellesmere Ice Shelf. Arctic, 10, 32-44

Hauke, J., Kossowski, T. (2011). Comparison of values of Pearson's and Spearman's correlation coefficients on the same sets of data. Quaestiones Geographicae, 30, 87-93. DOI 10.2478/v10117-011-0021-1

Holdsworth, G. (1987). The surface waveforms on the Ellesmere Island ice shelves and ice islands, in: Pilkington, G.R., Danielewicz, B.W. (Eds.). Presented at the 
Workshop on Extreme Ice Features, National Research Council of Canada, Banff, Alberta, 385-403

Hossain, M., Yackel, J., Dabboor, M., Fuller, M.C. (2014). Application of a threecomponent scattering model over snow-covered first-year sea ice using polarimetric C-band SAR data. International Journal of Remote Sensing, 35, 1786-1803. DOI 10.1080/01431161.2013.879345

Hsu, C., Chang, C., Lin, C. (2010). A practical guide to support vector classification. http://www.csie.ntu.edu.tw/ cjlin/papers/guide/guide.pdf

Huiying, L., Huadong, G., Lu, Z. (2014). Sea ice classification using dual polarization SAR data. IOP Conference Series: Earth and Environmental Science, 17, 012115. DOI 10.1088/1755-1315/17/1/012115

Improved Outcomes Software. (2014). Tutorial 9: Introduction. http://www.improvedoutcomes.com/docs/WebSiteDocs/Introduction/Tutorials/Tuto rial_9_Support_Vector_Machines/Tutorial_9_Introduction.htm

Iwashita, A., Qong, M. (2006). Consideration of the correlation between beta-angle and lineament patterns by using polarimetric SAR images. Presented at the International Geoscience and Remote Sensing Symposium, IEEE, Denver, Coloradro, 15331536. DOI 10.1109/IGARSS.2006.395

Jeffries, M.O. (2002). Ellesmere island ice shelves and ice islands, in: Williams, R.S., Ferrigno, J.G. (Eds.), Satellite Image Atlas of Glaciers of the World: North America, Satellite Image Atlas of Glaciers of the World. United States Geological Survey, Washington, J147-J164

Jeffries, M.O., Sackinger, W.M. (1990). Ice island detection and characterization with airborne synthetic aperature radar. Journal of Geophysical Research, 95, 5371-5377

Johannessen, O.M., Alexandrov, V., Frolov, I.Y., Sandven, S., Pettersson, L.H., Bobylev, L.P., Kloster, K., Smirnov, V.G., Mironov, Y.U., Babich, N.G. (2006). Remote Sensing of Sea Ice in the Northern Sea Route: Studies and Applications. Springer, Berlin

Johnston, M., Timco, G.W. (2008). Understanding and identifying old ice in summer. Canadian Hydraulics Centre, National Research Council Canada, Ottawa, 236. book

Joseph, G. (2005). Fundamentals of Remote Sensing, 2nd ed. Universities Press, 486

Judson, B. (2010). Trends in canadian arctic shipping traffic-myths and rumours. Presented at the The 20th International Offshore (Ocean) and Polar Engineering Conference \& Exhibition, Beijing, China

Kelso, T.S. (2015). CelesTrak. http://celestrak.com/

Kindt, R. (2005). Tree Diversity Analysis: A Manual and Software for Common Statistical Methods for Ecological and Biodiversity Studies. World Agroforestry Centre, Nairobi, Kenya, 207 
Koenig, L.S., Greenaway, K.R., Dunbar, M., Hattersley-Smith, G. (1952). Arctic ice islands. Arctic, 5, 67-103

Koizumi, K., Okamoto, N., Seo, T. (2009). On Jarque-Bera tests for assessing multivariate normality. Journal of Statistics: Advances in Theory and Applications, 1, 207-220

Krogager, E. (1990). New decomposition of the radar target scattering matrix. Electronics Letters, 26, 1525-1527

Leblon, B. (2012). Radar polarimetry. Presented at the course: Advanced Studies in Radar Polarimetry (FOR6304), University of New Brunswick

Lee, J.-S., Grunes, R., Ainsworth, T.L., Du, L.-J., Schuler, D.L., Cloude, S.R. (1999). Unsupervised classification using polarimetric decomposition and the complex Wishart classifier. IEEE Transactions on Geoscience and Remote Sensing, 37(5), 2249-2258

Lee, J.S., Pottier, E. (2009). Polarimetric radar imaging: from basics to applications. CRC Press, Boca Raton, Florida, 438. DOI 10.1080/01431161.2010.519925

Legendre, P., Legendre, L. (1998). Numerical Ecology, 2nd ed, Developments in Environmental Modelling. Elsevier, Amsterdam, 853.

Leigh, S., Zhijie Wang, Clausi, D.. (2014). Automated ice-water classification using dual polarization SAR satellite imagery. Geoscience and Remote Sensing, IEEE Transactions on, 52, 5529-5539. DOI 10.1109/TGRS.2013.2290231

Lewis, M., Necsoiu, M. (2011). Seeing sea ice. Technology Today, 34, 2-5

Liu, C., Frazier, P., Kumar, L. (2007). Comparative assessment of the measures of thematic classification accuracy. Remote Sensing of Environment, 107, 606-616

Liu, X., Li, Y., Gao, W., Xiao, L. (2010). Double polarization SAR image classification based on object-oriented technology. Journal of Geographic Information System, 2, 113-119. DOI 10.4236/jgis.2010.22017

Lobo, A., Legendre, P., Rebollar, J.L.G., Carreras, J., Ninot, J.-M. (2004). Land cover classification at a regional scale in Iberia: separability in a multi-temporal and multi-spectral data set of satellite images. International Journal of Remote Sensing, 25, 205-213. DOI 10.1080/0143116031000116435

Lopez-Martinez, C., Pottier, E., Cloude, S.R. (2005). Statistical assessment of eigenvector-based target decomposition theorems in radar polarimetry. IEEE Transactions on Geoscience and Remote Sensing, 43, 2058-2074. DOI 10.1109/TGRS.2005.853934

MacDonald, Dettwiler and Associates. (2013). RS2 product description. http://gs.mdacorporation.com/includes/documents/521238_RS2_Product_Description_Iss1-10.pdf

Maitra, S., Gartley, M.G., Faulring, J., Kerekes, J.P. (2013). Characterization of basic scattering mechanisms using laboratory based polarimetric synthetic aperture radar 
imaging. Geoscience and Remote Sensing Symposium (IGARSS), 2013 IEEE International, 4479-4482. DOI 10.1109/IGARSS.2013.6723830

Majd, M.S. (2014). High-resolution polarimetric sar image classification on urban areas (PhD, Department of Earth Sciences). Conservatoire National des Arts et Metiers, 219. https://tel.archives-ouvertes.fr/tel-01126995

Manning, C.D., Raghavan, P., Schütze, H. (2008). Support vector machines and machine learning on documents, in: Introduction to Information Retrieval. Cambridge University Press, Cambridge, England, 293-320. DOI 10.1017/CBO9780511809071

Mansouri, B., Shinozuka, M., Entezari, I., Motagh, M. (2010). Fully polarimetric SAR parameters and correlation with target-sensor orientation and building height. Presented at the 8th International Workshop on Remote Sensing for Disaster Management, Tokyo Institute of Technology, Tokyo, Japan, 1-8

Mansourpour, M., Rajabi, M.A., Blais, J.A.R. (2006). Effects and performance of speckle noise reduction filters on active radar and SAR images, in: Proceedings of the ISPRS Ankara Workshop. Ankara, Turkey

Ma, Q., Wang, J., Shang, J., Peng Wang. (2013). Assessment of multi-temporal RADARSAT-2 polarimetric SAR data for crop classification in an urban/rural fringe area. Presented at the Second International Conference on AgroGeoinformatics, Fairfax, Virginia, 314-319. DOI 10.1109/ArgoGeoinformatics.2013.6621928

Marino, A. (2010). A new target detector based on geometrical perturbation filters for polarimetric synthetic aperture radar (POL-SAR) (PhD, School of Geosciences). University of Edinburgh, Edinburgh, Scotland, 323

Marshall, E.W. (1955). Structural and stratigraphic studies of the northern Ellesmere Ice Shelf. Arctic, 8, 109-114

Massonnet, D., Souyris, J.C. (2008). Imaging with Synthetic Aperture Radar. EFPL Press, Lausanne, Switzerland, 281

Mather, P., Koch, M. (2011). Computer Processing of Remotely-Sensed Images: An Introduction. Wiley, 460.

McDonald, J.H. (2008). Handbook of Biological Statistics, 2nd ed. Sparky House Publishing, Baltimore, Maryland, 317

Mellor, M., Kovacs, A., Hnatiuk, J. (1977). Destruction of ice islands with explosives, in: Fourth International Conference on Port and Ocean Engineering Under Arctic Conditions, Sept. 26-30, 1977. Memorial University, St. John’s, Newfoundland, Canada, 1-13

Mishchenko, M.I., Hovenier, J.W., Travis, L.D. (Eds.). (2000). Light Scattering by Nonspherical Particles: Theory, Measurements, and Applications. Academic Press, San Diego, California, 690. 
Morio, J., Refregier, P., Goudail, F., Dubois-Fernandez, P.C., Dupuis, X. (2009). A characterization of Shannon entropy and Bhattacharyya measure of contrast in polarimetric and interferometric SAR image, in: Proceedings of the IEEE. 10971108. DOI 10.1109/JPROC.2009.2017107

Mortimer, C.A., Copland, L., Mueller, D.R. (2012). Volume and area changes of the Milne Ice Shelf, Ellesmere Island, Nunavut, Canada, since 1950. Journal of Geophysical Research, 117, F04011. DOI 10.1029/2011JF002074

Mueller, D.R., Crawford, A., Copland, L., VanWychen, W. (2013). Ice Island and Iceberg Fluxes from Canadian High Arctic Sources (Contract Report No. Final Draft Report). Northern Transportation Assessment Initiative, Innovation Policy Branch, Transport Canada, Ottawa, 23

NASA. (2010). NASA Earth Observatory. http://earthobservatory.nasa.gov/IOTD/view.php?id=45306

Natrella, M. (2012). Levene test for equality of variances, in: Croarkin, C., Tobias, P., Filliben, J.J., Hembree, B., Guthrie, W., Trutna, L., Prins, J. (Eds.), NIST/SEMATECH E-Handbook of Statistical Methods. National Institute of Standards and Technology

Neumann, M., Ferro-Famil, L., Pottier, E. (2009). A general model-based polarimetric decomposition scheme for vegetated areas, in: Proceedings of PollnSAR Workshop. Frascati, Italy, 1-8

Ofungwu, J. (2014). Statistical Applications for Environmental Analysis and Risk Assessment. Wiley, Hoboken, New Jersey, 648.

Oliver, C., Quegan, S. (2004). Understanding Synthetic Aperture Radar Images. SciTech Publishing, Raleigh, North Carolina, 464

Onstott, R.G. (1992). SAR and scatterometer signatures of sea ice, in: Carsey, F.D. (Ed.), Microwave Remote Sensing of Sea Ice. American Geophysical Union, Washington, D.C., 73-104. DOI 10.1029/GM068p0073

Onstott, R.G., Shuchman, R.A. (2004). SAR measurements of sea ice, in: Jackson, C.R., Apel, J.R. (Eds.), Synthetic Aperture Radar Marine User’s Manual. National Oceanic and Atmospheric Administration, Washington, DC, 81-115

Ouarzeddine, M., Souissi, B., Belhadj-Aissa, A. (2007). Unsupervised classification using Wishart classifier, in: Proceedings of POLinSAR Workshop. Frascati, Italy, 1-6

Paradella, W.R., Santos, A.R. dos, Veneziani, P., de Morais, M.C. (2000). Synthetic Aperture Radar for geological applications in the moist tropics: experiences from the Brazilian Amazon region. Brazilian Journal of Geology, 30, 538-542

Pasher, J., King, D.J. (2010). Multivariate forest structure modelling and mapping using high resolution airborne imagery and topographic information. Remote Sensing of Environment, 114, 1718-1732. DOI 10.1016/j.rse.2010.03.005

Praks, J. (2012). Radar polarimetry and interferometry for remote sensing of boreal forest 
(PhD, Department of Radio Science and Engineering). Aalto University, 112

Pritchard, M.E. (2006). InSAR, a tool for measuring Earth's surface deformation. Physics Today, 59, 68-69

QGIS Development Team. (2014). QGIS Geographic Information System. Open Source Geospatial Foundation. http://qgis.osgeo.org

Réfrégier, P., Morio, J. (2006). Shannon entropy of partially polarized and partially coherent light with Gaussian fluctuations. Journal of the Optical Society of America, 23, 3036-3044. DOI 10.1364/JOSAA.23.003036

Richards, J.A. (2012). Remote Sensing Digital Image Analysis: An Introduction. Springer, Berlin, 496

Richards, J.A. (2009). Remote Sensing with Imaging Radar. Springer, Berlin, 373. DOI 10.1007/978-3-642-02020-9

Richer-McCallum, M. (2015). Discriminating different ice types with synthetic aperture radar (SAR) satellite imagery along the northern coast of Ellesmere Island, Nunavut, Canada (M.Sc., Department of Geography and Environmental Studies). Carleton University, Ottawa, Canada

Sabins, F.F. (2007). Remote Sensing: Principles and Applications, 3rd ed. Waveland Press, 494

Sackinger, W.M., Jeffries, M.O., Li, F., Lu, M. (1991). Ice island creation, drift, recurrences, mechanical properties, and interactions with Arctic offshore oil production structures (Final Report for the U.S. Department of Energy). University of Alaska, Fairbanks, Alaska, 1-34

Scheuchl, B., Caves, R., Cumming, I., Staples, G. (2001). Automated sea ice classification using spaceborne polarimetric SAR data. Presented at the International Geoscience and Remote Sensing Symposium, IEEE, Sydney, Australia, 3117-3119

Scheuchl, B., Caves, R., Cumming, I., Staples, G. (2001). H/A/ $\alpha$-based classification of sea ice using SAR polarimetry, in: Proceedings of the 23rd Canadian Symposium on Remote Sensing. Quebec City, Canada, 21-24.

Scheuchl, B., Cumming, I., Hajnsek, I. (2005). Classification of fully polarimetric singleand dual-frequency SAR data of sea ice using the Wishart statistics. Canadian Journal of Remote Sensing, 31, 61-72

Scheuchl, B., Hajnsek, I., Cumming, I. (2002). Model-based classification of polarimetric SAR sea ice data. Presented at the International Geoscience and Remote Sensing Symposium, IEEE, Toronto, Ontario, Canada, 1521-1523. DOI 10.1109/IGARSS.2002.1026168

Schumacker, R.E. (2014). Learning Statistics Using R. SAGE Publications, University of Alabama, Tuscaloosa, 648.

scikit-image developers. (v0.11dev). Module: segmentation - skimage v0.11dev docs. 
http://scikit-image.org/docs/dev/api/skimage.segmentation.html

scikit-learn developers. (v0.16.0). RBF SVM parameters - scikit-learn 0.16.0 documentation. http://scikitlearn.org/stable/auto_examples/svm/plot_rbf_parameters.html

scikit-learn developers. (n.d.). 3.2. Support Vector Machines - scikit-learn 0.11 documentation. http://scikit-learn.org/stable/modules/svm.html

Serreze, M.C., Barry, R.G. (2011). Processes and impacts of Arctic amplification: A research synthesis. Global and Planetary Change, 77, 85-96. DOI 10.1016/j.gloplacha.2011.03.004

Shokr, M., Sinha, N. (2015). Sea Ice: Physics and Remote Sensing. Wiley, 600. DOI 10.1002/9781119028000

Similä, M., Mäkynen, M., Heiler, I. (2010). Comparison between C band synthetic aperture radar and 3-D laser scanner statistics for the Baltic Sea ice. Journal of Geophysical Research: Oceans, 115, 1-18. DOI 10.1029/2009JC005970

Simpson, G. (2011). [R-sig-eco] How to choose between RDA and CCA? https://stat.ethz.ch/pipermail/r-sig-ecology/2011-August/002280.html

Small, D., Miranda, N., Meier, E. (2009). A revised radiometric normalisation standard for SAR. Presented at the International Geoscience and Remote Sensing Symposium, IEEE, Cape Town, South Africa, IV-566 - IV-569. DOI 10.1109/IGARSS.2009.5417439

Šmilauer, P., Lepš, J. (2014). Multivariate Analysis of Ecological Data using CANOCO 5. Cambridge University Press, Cambridge, UK, 373

Solomon, S., Qin, D., Manning, M., Chen, Z., Marquis, M., Averyt, K.B., Tignor, M., Miller, H.L. (2007). Climate Change 2007: The Physical Science Basis. Contribution of Working Group I to the Fourth Assessment Report of the Intergovernmental Panel on Climate Change. Cambridge University Press, Cambridge, UK, 996

Spedding, L.G. (1977). Ice island count, southern Beaufort Sea 1976 (No. IPRT-21ME77). Arctic Petroleum Opel. Association, Calgary, Alberta, 46

Steele, J.H., Thorpe, S.A., Turekian, K.K. (2009). Elements of Physical Oceanography: A derivative of the Encyclopedia of Ocean Sciences. Elsevier Science, London, UK, 660

Stehman, S. (1996). Estimating the kappa coefficient and its variance under stratified random sampling. Photogrammetric Engineering and Remote Sensing, 62, 401-407

Torontow, V., King, D. (2011). Forest complexity modelling and mapping with remote sensing and topographic data: a comparison of three methods. Canadian Journal of Remote Sensing, 37, 387-402. DOI 10.5589/m11-047

Touzi, R. (2007). Target scattering decomposition in terms of roll-invariant target parameters. IEEE Transactions on Geoscience and Remote Sensing, 45, 73-84. 
DOI 10.1109/TGRS.2006.886176

Turner, D., Woodhouse, I.H. (2012). An icon-based synoptic visualization of fully polarimetric radar data. Remote Sensing, 4, 648-660. DOI 10.3390/rs4030648

Van der Sanden, J.J., Drouin, H. (2011). Satellite SAR observations of river ice cover: A RADARSAT-2 (C-band) and ALOS PALSAR (L-band) comparison, in:

Proceedings of the 16th Workshop on River Ice. Winnipeg, Manitoba, 18-22

Vant, M.R., Ramseier, R.O., Makios, V. (1978). The complex-dielectric constant of sea ice at frequencies in the range 0.1-40 GHz. Journal of Applied Physics, 49, 12641280. DOI 10.1063/1.325018

van Zyl, J.J., Arii, M., Kim, Y. (2011). Model-based decomposition of polarimetric SAR covariance matrices constrained for nonnegative eigenvalues. IEEE Transactions on Geoscience and Remote Sensing, 49, 3452-3459. DOI 10.1109/TGRS.2011.2128325

van Zyl, J.J., Kim, Y. (2011). Synthetic aperture radar polarimetry. Wiley, Hoboken, New Jersey, 288. DOI 10.1002/9781118116104

Wagner, P. (2012). Machine Learning with OpenCV2. http://www.bytefish.de/pdf/machinelearning.pdf

White, A., Mueller, D., Copland, L. (2015). Reconstructing hydrographic change in Petersen Bay, Ellesmere Island, Canada, inferred from SAR imagery. Remote Sensing of Environment, 165, 1-13

Wilcox, R.R. (2003). Applying Contemporary Statistical Techniques. Elsevier Science, San Diego, California, 608. DOI 10.1198/tech.2004.s233

Xiao-xia, S., Ji-xian, Z., Zheng-jun, L., Zheng, Z. (2006). Classification from airborne SAR data enhanced by optical image using an object-oriented approach, in: Proceedings of Remote Sensing: From Pixels to Processes. Presented at the ISPRS Commission VII Symposium, Enschede, The Netherlands, 1-3

Yackel, J.J., Barber, D.G. (2000). Melt ponds on sea ice in the Canadian Archipelago 2. On the use of RADARSAT-1 synthetic aperture radar for geophysical inversion. Journal of Geophysical Research, 105, 22061-22070

Yackel, J.J., Barber, D.G., Papakyriakou, T.N., Breneman, C. (2007). First-year sea ice spring melt transitions in the Canadian Arctic archipelago from time-series synthetic aperture radar data, 1992-2002. Hydrological Processes, 21, 253-265. DOI 10.1002/hyp.6240

Yonezawa, C., Watanabe, M., Saito, G. (2012). Polarimetric decomposition analysis of ALOS PALSAR observation data before and after a landslide event. Remote Sensing, 4, 2314-2328

Yong, S.Y. (2004). Radar polarimetry (M.Sc, Combat Systems Technology). Naval Postgraduate School, Monterey, California, 89

Young, I.R. (1999). Wind Generated Ocean Waves. Elsevier Science, 288 
Yu, H., Kim, S. (2012). SVM tutorial - classification, regression and ranking, in: Rozenberg, G., Bäck, T., Kok, J. (Eds.), Handbook of Natural Computing. Springer Berlin Heidelberg, 479-506. DOI 10.1007/978-3-540-92910-9_15

Zhang, Y., Wu, L., Wei, G. (2009). A new classifier for polarimetric SAR images. Progress In Electromagnetics Research, 94, 83-104

Zuur, A.F. (2007). Analysing Ecological Data, Statistics for Biology and Health. Springer, New York, 672. DOI 10.1007/978-0-387-45972-1 


\section{Appendix A}

\subsection{Derivation of Coherency and Covariance Matrices from the Sinclair Matrix}

Given four polarizations in fully polarimetric radar, these elements may be expressed as the Sinclair or Scattering matrix: $S=\left[\begin{array}{ll}S_{H H} & S_{H V} \\ S_{V H} & S_{V V}\end{array}\right]$ where $S_{V H}=S_{H V}$ due to reciprocity (Richards, 2009; Shokr and Sinha, 2015). This reciprocity holds true except in cases where rotation of polarization (Faraday rotation) occurs in the ionosphere, most commonly with lower frequencies of L- and P-band. Generally at the higher frequencies of C- and X-band used in SAR imaging, Faraday rotation is minor and thus, reciprocity holds true (Richards, 2009). . Each element is a complex number expressing both the amplitude and phase of the backscattered returns. Converting this Sinclair matrix to a scattering vector allows for more advanced classification techniques:

$$
k=\left[\begin{array}{l}
S_{H H} \\
S_{H V} \\
S_{V H} \\
S_{V V}
\end{array}\right]=\left[\begin{array}{llll}
S_{H H} & S_{H V} & S_{V H} & S_{V V}
\end{array}\right]^{T}
$$

which may be reduced to, due to reciprocity, $\left[\begin{array}{lll}S_{H H} & S_{H V} & S_{V V}\end{array}\right]^{T}$, which is often rewritten as $\left[\begin{array}{lll}S_{H H} & \sqrt{2} S_{H V} & S_{V V}\end{array}\right]^{T}$ such that the span of the entire vector remains constant through the reciprocity reduction (Richards, 2009). The covariance matrix may be determined with 


$$
\begin{aligned}
& \because C=\vec{k}_{l} \cdot \vec{k}_{l}^{+} \\
& \text {where } \vec{k}_{l} \text { is the scattering vector, } \vec{k}_{l}=\left[\begin{array}{lll}
S_{H H} \sqrt{2} S_{H V} & S_{V V}
\end{array}\right]^{T} \\
& C=\left[\begin{array}{ll}
S_{H H} \sqrt{2} S_{H V} & S_{V V}
\end{array}\right]^{T} \cdot\left(\left[\begin{array}{ll}
S_{H H} \sqrt{2} S_{H V} & S_{V V}
\end{array}\right]^{T}\right)^{+} \\
& C=\left[\begin{array}{l}
S_{H H} \\
\sqrt{2} S_{H V} \\
S_{V V}
\end{array}\right] \cdot\left[\begin{array}{lll}
S_{H H}^{*} & \sqrt{2} S_{H V}^{*} & S_{V V}^{*}
\end{array}\right] \\
& \begin{array}{c}
C=\left[\begin{array}{ccc}
S_{H H} S_{H H}^{*} & \sqrt{2} S_{H V}^{*} S_{H H} & S_{H H} S_{V V}^{*} \\
\sqrt{2} S_{H V} S_{H H}^{*} & 2 S_{H V} S_{H V}^{*} & \sqrt{2} S_{H V} S_{V V}^{*} \\
S_{V V} S_{H H}^{*} & \sqrt{2} S_{H V}^{*} S_{V V} & S_{V V} S_{V V}^{*}
\end{array}\right] \\
C=\left[\begin{array}{ccc}
\left|S_{H H}\right|^{2} & \sqrt{2} S_{H V}^{*} S_{H H} & S_{H H} S_{V V}^{*} \\
\sqrt{2} S_{H V} S_{H H}^{*} & 2\left|S_{H V}\right|^{2} & \sqrt{2} S_{H V} S_{V V}^{*} \\
S_{V V} S_{H H}^{*} & \sqrt{2} S_{H V}^{*} S_{V V} & \left|S_{V V}\right|^{2}
\end{array}\right]
\end{array}
\end{aligned}
$$

similarly, for the coherency matrix with reciprocity (Richards, 2009),

$$
\begin{aligned}
& \because T=\vec{k}_{p} \cdot \overrightarrow{k_{p}^{+}} \\
& \text {where } \vec{k}_{p} \text { is the Pauli basis target vector, } \vec{k}_{p}=\frac{1}{\sqrt{2}}\left[\begin{array}{lll}
S_{H H}+S_{V V} & S_{H H}-S_{V V} & 2 S_{H V}
\end{array}\right]^{T}
\end{aligned}
$$$$
T=\frac{1}{\sqrt{2}}\left[\begin{array}{lll}
S_{H H}+S_{V V} & S_{H H}-S_{V V} & 2 S_{H V}
\end{array}\right]^{T} \cdot\left(\frac{1}{\sqrt{2}}\left[\begin{array}{lll}
S_{H H}+S_{V V} & S_{H H}-S_{V V} & 2 S_{H V}
\end{array}\right]^{T}\right)^{+}
$$$$
T=\frac{1}{\sqrt{2}}\left[\begin{array}{l}
S_{H H}+S_{V V} \\
S_{H H}-S_{V V} \\
2 S_{H V}
\end{array}\right] \cdot\left(\frac{1}{\sqrt{2}}\left[\begin{array}{l}
S_{H H}+S_{V V} \\
S_{H H}-S_{V V} \\
2 S_{H V}
\end{array}\right]\right)
$$$$
T=\frac{1}{\sqrt{2}}\left[\begin{array}{l}
S_{H H}+S_{V V} \\
S_{H H}-S_{V V} \\
2 S_{H V}
\end{array}\right] \cdot\left(\frac{1}{\sqrt{2}}\left[\begin{array}{lll}
S_{H H}^{*}+S_{V V}^{*} & S_{H H}^{*}-S_{V V}^{*} & 2 S_{H V}^{*}
\end{array}\right]\right)^{+}
$$$$
T=\frac{1}{2}\left[\begin{array}{lll}
\left(S_{H H}+S_{V V}\right)\left(S_{H H}^{*}+S_{V V}^{*}\right) & \left(S_{H H}+S_{V V}\right)\left(S_{H H}^{*}-S_{V V}^{*}\right) & 2\left(S_{H H}+S_{V V}\right) S_{H V}^{*} \\
\left(S_{H H}-S_{V V}\right)\left(S_{H H}^{*}+S_{V V}^{*}\right) & \left(S_{H H}-S_{V V}\right)\left(S_{H H}^{*}-S_{V V}^{*}\right) & 2\left(S_{H H}-S_{V V}\right) S_{H V}^{*} \\
2 S_{H V}\left(S_{H H}^{*}+S_{V V}^{*}\right) & 2 S_{H V}\left(S_{H H}^{*}-S_{V V}^{*}\right) & 4 S_{H V} S_{H V}^{*}
\end{array}\right]
$$ 


\section{Appendix B}

\subsection{SAR Variables Used in this Study}

Table 8.1: SAR variables used in this study

\begin{tabular}{|c|c|c|}
\hline $\begin{array}{c}\text { SAR Variable as named in } \\
\text { PolSARpro } \\
\end{array}$ & Brief description & Supplementary Reference \\
\hline A11 & Amplitude of $\mathrm{HH}$ & \\
\hline A12 & Amplitude of HV & \\
\hline A21 & Amplitude of VH & \\
\hline A22 & Amplitude of VV & \\
\hline alpha1 & $\alpha$ angle 1 & (van Zyl and Kim, 2011) \\
\hline alpha2 & $\alpha$ angle 2 & (van Zyl and Kim, 2011) \\
\hline alpha3 & $\alpha$ angle 3 & (van Zyl and Kim, 2011) \\
\hline alpha & $\alpha$ angle & (van Zyl and Kim, 2011) \\
\hline $\begin{array}{r}\text { anisotropy12 } \\
\text { anisotropy }\end{array}$ & $\begin{array}{l}\text { Anisotropy of higher eigenvalues } \\
\text { Anisotropy }\end{array}$ & (Guillaso et al., 2015; Majd, 2014) \\
\hline anisotropy_lueneburg & Lueneburg Anisotropy & (Majd, 2014) \\
\hline asymmetry & Polarization Asymmetry & (Majd, 2014) \\
\hline beta1 & $\beta$ angle 1 & (van Zyl and Kim, 2011) \\
\hline beta2 & $\beta$ angle 2 & (van Zyl and Kim, 2011) \\
\hline beta3 & $\beta$ angle 3 & (van Zyl and Kim, 2011) \\
\hline beta & $\beta$ angle & (van Zyl and Kim, 2011) \\
\hline C11 & Covariance matrix element 1,1 & \\
\hline C12_imag & Imaginary component of the & \\
\hline & covariance matrix element 1,2 & \\
\hline C12_real & $\begin{array}{l}\text { Real component of the covariance } \\
\text { matrix element } 1,2\end{array}$ & \\
\hline C13_imag & Imaginary component of the & \\
\hline & covariance matrix element 1,3 & \\
\hline C13_real & $\begin{array}{l}\text { Real component of the covariance } \\
\text { matrix element } 1,3\end{array}$ & \\
\hline C22 & Covariance matrix element 2,2 & \\
\hline C23_imag & Imaginary component of the & \\
\hline & covariance matrix element 2,3 & \\
\hline C23_real & $\begin{array}{l}\text { Real component of the covariance } \\
\text { matrix element } 2,3\end{array}$ & \\
\hline C33 & Covariance matrix element 3,3 & \\
\hline combination_1mH1mA & $\begin{array}{l}\text { Combination (1-entropy)(1- } \\
\text { anisotropy) }\end{array}$ & (Marino, 2010) \\
\hline combination_1mHA & $\begin{array}{l}\text { Combination (1- } \\
\text { entropy)anisotropy }\end{array}$ & (Marino, 2010) \\
\hline combination_H1mA & $\begin{array}{l}\text { Combination entropy(1- } \\
\text { anisotropy) }\end{array}$ & (Marino, 2010) \\
\hline combination_HA & Combination entropy $\mathrm{x}$ anisotropy & (Marino, 2010) \\
\hline delta1 & Delta 1 , related to phase relations & (Yonezawa et al., 2012) \\
\hline delta2 & Delta 2, related to phase relations & (Yonezawa et al., 2012) \\
\hline
\end{tabular}




\section{SAR Variable as named in} PolSARpro

Brief description

delta3 Delta 3, related to phase relations

delta Delta, related to phase relations

derd Double-bounce Eigenvalue Relative Difference

derd_norm Normalized Double-bounce Eigenvalue Relative Difference entropy Entropy

entropy_shannon_I Shannon Entropy, intensity entropy_shannon Shannon Entropy

entropy_shannon_I_norm Normalized Shannon Entropy, intensity

entropy_shannon_norm Normalized Shannon Entropy

entropy_shannon_P Shannon Entropy, polarization

entropy_shannon_P_norm Normalized Shannon Entropy, polarization

Freeman_Dbl Freeman-Durden Double-bounce component

Freeman_Odd Freeman-Durden Odd-bounce component

Freeman_Vol Freeman-Durden Volume component

gamma1 $\gamma 1$, related to phase relations

gamma2 $\gamma 2$, related to phase relations

gamma3 $\gamma 3$, related to phase relations

gamma $\gamma$, related to phase relations

I11 Intensity of $\mathrm{HH}$

I12 Intensity of $\mathrm{HV}$

I21 Intensity of $\mathrm{VH}$

I22 Intensity of VV

Krogager_Kd Krogager decomposition doublebounce component

Krogager_Kh Krogager decomposition helicalbounce component

Krogager_Ks Krogager decomposition singlebounce component

11 Eigenvalues 1

12 Eigenvalues 2

13 Eigenvalues 3

lambda $\lambda$

Neumann_delta_mod Neumann $\delta$ magnitude of particle anisotropy

Neumann_delta_pha Neumann $\delta$ phase of particle anisotropy

Neumann_psi Neumann $\Psi$, particle orientation angle

Neumann_tau Neumann , degree of particle orientation randomness

p1 Probabilities 1

p2 Probabilities 2
Supplementary Reference

(Yonezawa et al., 2012)

(Yonezawa et al., 2012)

(Lee and Pottier, 2009)

(Lee and Pottier, 2009)

(Agashe, 2013)

(Morio et al., 2009)

(Morio et al., 2009)

(Morio et al., 2009)

(Morio et al., 2009)

(Morio et al., 2009)

(Morio et al., 2009)

(Freeman and Durden, 1998)

(Freeman and Durden, 1998)

(Freeman and Durden, 1998)

(Yonezawa et al., 2012)

(Yonezawa et al., 2012)

(Yonezawa et al., 2012)

(Yonezawa et al., 2012)

(Krogager, 1990)

(Krogager, 1990)

(Krogager, 1990)

(Lopez-Martinez et al., 2005)

(Lopez-Martinez et al., 2005)

(Lopez-Martinez et al., 2005)

(Lopez-Martinez et al., 2005)

(Neumann et al., 2009)

(Neumann et al., 2009)

(Neumann et al., 2009)

(Neumann et al., 2009)

(Massonnet and Souyris, 2008;

Oliver and Quegan, 2004)

(Massonnet and Souyris, 2008; 


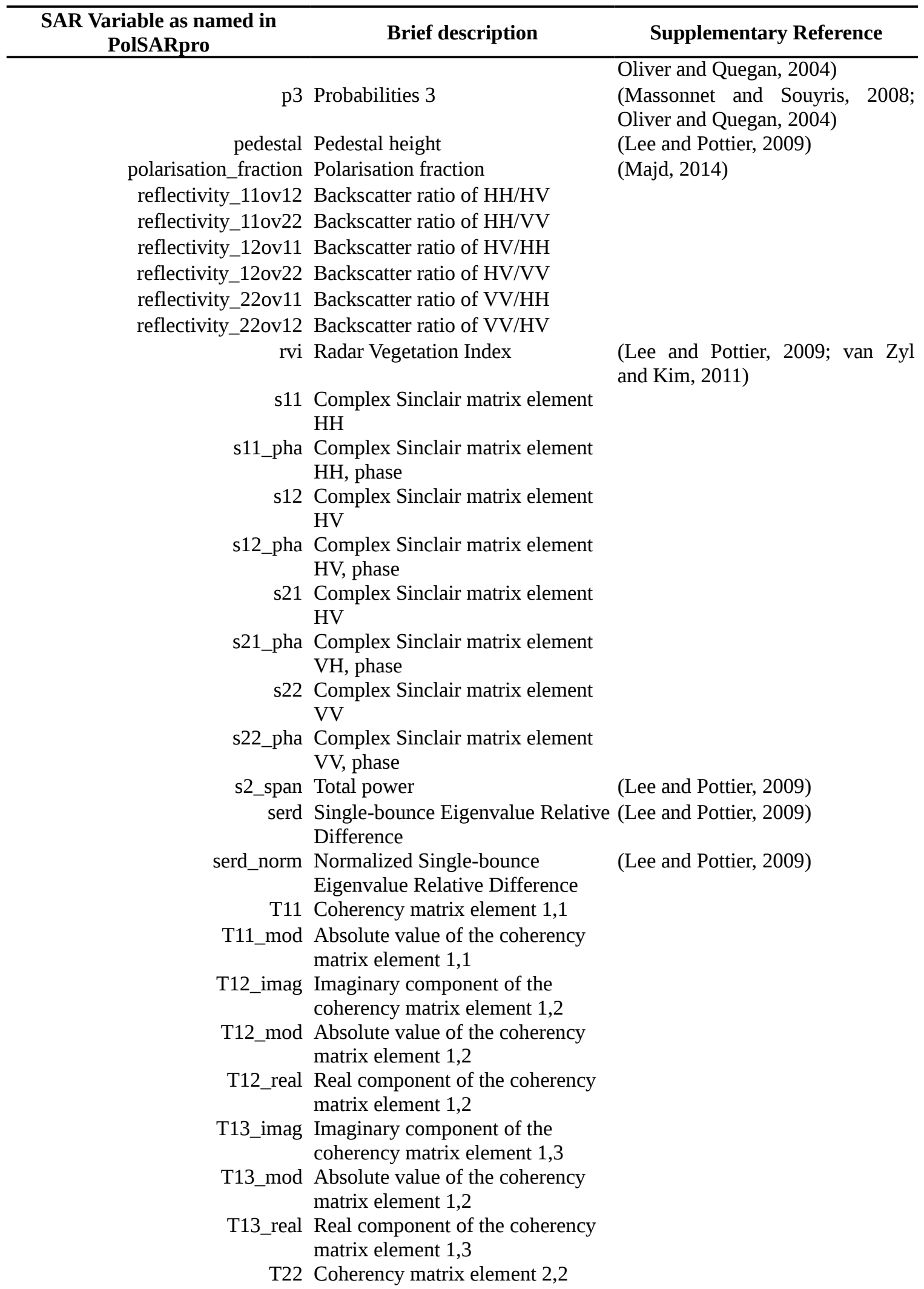




\begin{tabular}{|c|c|c|}
\hline $\begin{array}{c}\text { SAR Variable as named in } \\
\text { PolSARpro }\end{array}$ & Brief description & Supplementary Reference \\
\hline T22_mod & $\begin{array}{l}\text { Absolute value of the coherency } \\
\text { matrix element } 2,2\end{array}$ & \\
\hline T23_imag & $\begin{array}{l}\text { Imaginary component of the } \\
\text { coherency matrix element } 2,3\end{array}$ & \\
\hline T23_mod & $\begin{array}{l}\text { Absolute value of the coherency } \\
\text { matrix element } 2,3\end{array}$ & \\
\hline T23_real & $\begin{array}{l}\text { Real component of the coherency } \\
\text { matrix element } 2,3\end{array}$ & \\
\hline T33 & Coherency matrix element 3,3 & \\
\hline T33_mod & $\begin{array}{l}\text { Absolute value of the coherency } \\
\text { matrix element } 3,3\end{array}$ & \\
\hline TSVM_alpha_s1 & $\begin{array}{l}\text { Touzi symmetric scattering type } \\
\text { magnitude for eigenvector } 1\end{array}$ & (Touzi, 2007) \\
\hline TSVM_alpha_s2 & $\begin{array}{l}\text { Touzi symmetric scattering type } \\
\text { magnitude for eigenvector } 2\end{array}$ & (Touzi, 2007) \\
\hline TSVM_alpha_s3 & $\begin{array}{l}\text { Touzi symmetric scattering type } \\
\text { magnitude for eigenvector } 3\end{array}$ & (Touzi, 2007) \\
\hline TSVM_alpha_s & $\begin{array}{l}\text { Touzi symmetric scattering type } \\
\text { magnitude }\end{array}$ & (Touzi, 2007) \\
\hline TSVM_phi_s1 & $\begin{array}{l}\text { Touzi symmetric scattering type } \\
\text { phase for eigenvector } 1\end{array}$ & (Touzi, 2007) \\
\hline TSVM_phi_s2 & $\begin{array}{l}\text { Touzi symmetric scattering type } \\
\text { phase for eigenvector } 2\end{array}$ & (Touzi, 2007) \\
\hline TSVM_phi_s3 & $\begin{array}{l}\text { Touzi symmetric scattering type } \\
\text { phase for eigenvector } 3\end{array}$ & (Touzi, 2007) \\
\hline TSVM_phi_s & $\begin{array}{l}\text { Touzi symmetric scattering type } \\
\text { phase }\end{array}$ & (Touzi, 2007) \\
\hline TSVM_psi1 & $\begin{array}{l}\text { Touzi Kennaugh-Huynen } \\
\text { orientation angle for eigenvector } 1\end{array}$ & (Touzi, 2007) \\
\hline TSVM_psi2 & $\begin{array}{l}\text { Touzi Kennaugh-Huynen } \\
\text { orientation angle for eigenvector } 2\end{array}$ & (Touzi, 2007) \\
\hline TSVM_psi3 & $\begin{array}{l}\text { Touzi Kennaugh-Huynen } \\
\text { orientation angle for eigenvector } 3\end{array}$ & (Touzi, 2007) \\
\hline TSVM_psi & $\begin{array}{l}\text { Touzi Kennaugh-Huynen } \\
\text { orientation angle }\end{array}$ & (Touzi, 2007) \\
\hline TSVM_tau_m1 & $\begin{array}{l}\text { Touzi Kennaugh-Huynen helicity } \\
\text { for eigenvector } 1\end{array}$ & (Touzi, 2007) \\
\hline TSVM_tau_m2 & $\begin{array}{l}\text { Touzi Kennaugh-Huynen helicity } \\
\text { for eigenvector } 2\end{array}$ & (Touzi, 2007) \\
\hline TSVM_tau_m3 & $\begin{array}{l}\text { Touzi Kennaugh-Huynen helicity } \\
\text { for eigenvector } 3\end{array}$ & (Touzi, 2007) \\
\hline TSVM_tau_m & Touzi Kennaugh-Huynen helicity & (Touzi, 2007) \\
\hline VanZyl3_Dbl & $\begin{array}{l}\text { Van Zyl Double-bounce } \\
\text { component }\end{array}$ & (van Zyl et al., 2011) \\
\hline VanZyl3_Odd & Van Zyl Single-bounce component & (van Zyl et al., 2011) \\
\hline VanZyl3_Vol & Van Zyl Volume component & (van Zyl et al., 2011) \\
\hline wishart_H_A_alpha_class_3 & $\begin{array}{l}\text { Wishart classification using } \\
\text { entropy, anisotropy and alpha }\end{array}$ & (Ouarzeddine et al., 2007) \\
\hline wishart_H_alpha_class_3 & $\begin{array}{l}\text { Wishart classification using } \\
\text { entropy, and alpha }\end{array}$ & (Ouarzeddine et al., 2007) \\
\hline
\end{tabular}




\subsection{SAR Images Used in this Study}

Table 8.2: Confounding variables for each of the training images

\begin{tabular}{|c|c|c|c|c|c|c|c|}
\hline \multirow{2}{*}{$\begin{array}{c}\text { Date } \\
\text { (GMT) }\end{array}$} & \multirow{2}{*}{$\begin{array}{l}\text { Time } \\
\text { (GMT) }\end{array}$} & \multirow[b]{2}{*}{$\Phi$ (deg) } & \multirow{2}{*}{$\begin{array}{c}\text { Temperature } \\
\text { (K) }\end{array}$} & \multicolumn{2}{|c|}{ Wind Speed (m/s) } & \multirow{2}{*}{$\theta$ (deg) } & \multirow{2}{*}{$\begin{array}{c}\text { Mismatch } \\
\text { between ice } \\
\text { chart \& } \\
\text { SAR image } \\
\text { (days) } \\
\end{array}$} \\
\hline & & & & u vector & v vector & & \\
\hline $2009 / 09 / 15$ & $14: 21: 28$ & 48.5 & 271.2 & 0.3 & -3.9 & 39.1 & 1 \\
\hline 2009/09/16 & $15: 32: 24$ & 61.7 & 267.5 & 5.1 & -0.7 & 19.5 & 2 \\
\hline 2009/09/24 & 23:17:39 & 45.6 & 266.9 & -1.9 & -1.1 & 33.3 & 3 \\
\hline 2009/09/27 & 15:11:33 & 69.8 & 268.0 & 3.6 & -0.5 & 23.2 & 1 \\
\hline 2009/09/27 & 23:30:11 & 55.5 & 268.8 & -1.3 & -2.8 & 36.3 & 1 \\
\hline 2009/10/08 & 23:09:10 & 71.7 & 256.4 & -0.7 & -2.0 & 26.7 & 3 \\
\hline 2009/10/09 & $14: 21: 44$ & 58.1 & 257.9 & 0.8 & -4.2 & 36.3 & 3 \\
\hline 2009/10/16 & 22:36:06 & 13.8 & 254.3 & -0.1 & -11.5 & 25.5 & 3 \\
\hline 2009/10/18 & $23: 17: 50$ & 5 & 258.7 & -4.8 & -11.5 & 36.3 & 1 \\
\hline 2009/10/22 & 23:00:56 & 18.4 & 256.6 & 1.0 & -2.5 & 28.9 & 3 \\
\hline $2009 / 10 / 24$ & $23: 42: 40$ & 28.6 & 250.9 & -1.8 & -0.6 & 40.0 & 2 \\
\hline 2009/10/25 & $14: 54: 55$ & 78 & 249.4 & 1.8 & -2.0 & 26.7 & 1 \\
\hline 2009/11/03 & $23: 50: 52$ & 75.8 & 254.0 & -1.4 & 1.1 & 38.2 & 1 \\
\hline 2009/11/04 & 15:03:30 & 60.1 & 255.5 & 1.0 & -2.3 & 23.2 & 2 \\
\hline 2009/11/04 & 23:21:39 & 63.2 & 244.6 & -2.1 & -0.8 & 30.0 & 2 \\
\hline 2009/11/08 & 23:05:17 & 7 & 248.6 & 2.3 & -6.3 & 32.2 & 1 \\
\hline 2009/11/09 & 14:17:11 & 48.5 & 247.1 & 4.8 & -2.3 & 39.1 & 0 \\
\hline 2009/11/12 & $22: 48: 25$ & 10.1 & 252.0 & -1.7 & 1.7 & 24.3 & 3 \\
\hline 2009/11/14 & 23:30:09 & 21.5 & 262.2 & -9.6 & 11.9 & 36.3 & 2 \\
\hline 2009/11/30 & $22: 23: 40$ & 17.1 & 248.7 & -3.0 & 2.5 & 24.3 & 0 \\
\hline $2009 / 12 / 25$ & $15: 15: 34$ & 85.6 & 242.6 & 0.0 & -3.2 & 20.8 & 3 \\
\hline 2009/12/25 & $23: 34: 26$ & 20.7 & 244.5 & -3.4 & -6.8 & 40.9 & 3 \\
\hline $2009 / 12 / 26$ & $14: 46: 23$ & 75.8 & 245.7 & -2.7 & -6.2 & 28.9 & 2 \\
\hline 2010/01/08 & 15:07:35 & 42.7 & 233.2 & -0.1 & 0.6 & 23.2 & 4 \\
\hline 2010/01/10 & 14:09:09 & 55.5 & 242.2 & 4.2 & -4.0 & 40.0 & 6 \\
\hline 2010/01/16 & 14:33:38 & 51.6 & 240.4 & 0.0 & 7.0 & 35.3 & 2 \\
\hline 2010/01/16 & $22: 52: 52$ & 5 & 237.5 & -1.7 & 7.4 & 33.2 & 2 \\
\hline 2010/01/18 & $15: 15: 22$ & 58.4 & 227.7 & -0.3 & 1.1 & 25.5 & 0 \\
\hline 2010/01/21 & 22:06:49 & 6.8 & 240.0 & -2.2 & -6.3 & 19.5 & 3 \\
\hline 2010/01/22 & $23: 17: 44$ & 22.4 & 240.4 & -2.3 & -8.3 & 37.2 & 4 \\
\hline 2010/01/23 & $22: 48: 30$ & 15.5 & 239.3 & -1.7 & -12.6 & 30.1 & 5 \\
\hline 2010/01/29 & 23:13:21 & 79.6 & 246.8 & 3.5 & -10.8 & 27.8 & 3 \\
\hline 2010/01/30 & $22: 44: 10$ & 64.1 & 248.7 & 6.4 & -8.7 & 19.5 & 2 \\
\hline 2010/01/31 & $00: 24: 22$ & 89 & 248.7 & 6.4 & -8.7 & 47.5 & 1 \\
\hline 2010/02/19 & $14: 41: 54$ & 57.5 & 240.9 & 0.4 & -6.6 & 33.3 & 4 \\
\hline 2010/02/19 & 23:01:18 & 0 & 240.3 & -0.7 & -5.1 & 37.2 & 4 \\
\hline 2010/03/06 & $00: 32: 47$ & 81.7 & 245.4 & -2.5 & -7.0 & 48.9 & 5 \\
\hline 2010/03/11 & 23:17:32 & 76.4 & 250.9 & -4.8 & 4.5 & 29.0 & 3 \\
\hline 2010/03/18 & 23:13:37 & 23.1 & 243.3 & -1.7 & -5.1 & 34.3 & 3 \\
\hline 2010/03/19 & $22: 44: 25$ & 21.6 & 251.6 & -3.5 & -16.7 & 26.7 & 4 \\
\hline 2010/03/29 & 22:52:33 & 66.5 & 253.3 & -4.2 & -1.3 & 21.9 & 0 \\
\hline
\end{tabular}




\begin{tabular}{|c|c|c|c|c|c|c|c|}
\hline \multirow[b]{2}{*}{$\begin{array}{l}\text { Date } \\
\text { (GMT) }\end{array}$} & \multirow[b]{2}{*}{$\begin{array}{l}\text { Time } \\
\text { (GMT) }\end{array}$} & \multirow[b]{2}{*}{$\Phi($ deg) } & \multirow[b]{2}{*}{$\begin{array}{c}\text { Temperature } \\
\text { (K) }\end{array}$} & \multicolumn{2}{|c|}{ Wind Speed (m/s) } & \multirow[b]{2}{*}{$\theta$ (deg) } & \multirow{2}{*}{$\begin{array}{c}\text { Mismatch } \\
\text { between ice } \\
\text { chart \& } \\
\text { SAR image } \\
\text { (days) }\end{array}$} \\
\hline & & & & u vector & $\mathbf{v}$ vector & & \\
\hline $2010 / 04 / 30$ & $23: 59: 37$ & 42.3 & 261.8 & -1.9 & -5.0 & 44.3 & 3 \\
\hline $2010 / 05 / 07$ & $23: 55: 24$ & 25.7 & 260.2 & -1.7 & -1.0 & 40.1 & 3 \\
\hline $2010 / 05 / 08$ & $23: 26: 10$ & 30.5 & 263.4 & -2.6 & -1.4 & 33.3 & 2 \\
\hline $2010 / 05 / 26$ & $14: 42: 27$ & 82 & 268.5 & -0.9 & 6.4 & 33.3 & 2 \\
\hline 2010/06/01 & 23:26:09 & 28.2 & 271.7 & -0.4 & -4.2 & 33.3 & 1 \\
\hline $2010 / 06 / 29$ & 23:09:25 & 27.4 & 277.9 & -0.8 & 2.1 & 26.7 & 1 \\
\hline $2010 / 07 / 20$ & $22: 56: 56$ & 5.6 & 272.4 & 4.6 & 3.9 & 25.6 & 1 \\
\hline 2010/09/11 & $23: 50: 48$ & 49.7 & 269.0 & 2.6 & -3.8 & 37.2 & 2 \\
\hline $2010 / 10 / 27$ & 14:51:42 & 85.6 & 260.5 & 0.7 & 1.8 & 20.8 & 2 \\
\hline $2011 / 01 / 16$ & 13:49:18 & 48.5 & 250.2 & 2.0 & -13.5 & 40.0 & 1 \\
\hline 2011/03/01 & $00: 31: 40$ & 39.8 & 245.5 & 5.5 & -12.1 & 43.5 & 1 \\
\hline $2011 / 03 / 16$ & $23: 25: 13$ & 5.8 & 248.9 & 2.6 & 6.2 & 23.2 & 2 \\
\hline 2011/05/12 & 00:31:42 & 40.6 & 261.1 & 1.1 & -2.7 & 43.5 & 3 \\
\hline 2011/09/07 & $21: 38: 24$ & 40.1 & 277.2 & -2.0 & -5.1 & 32.2 & 2 \\
\hline 2011/09/07 & $21: 38: 27$ & 42.7 & 277.2 & -2.0 & -5.1 & 32.2 & 2 \\
\hline 2011/09/12 & $22: 34: 18$ & 86.4 & 276.4 & 7.0 & -0.5 & 32.2 & 0 \\
\hline 2011/09/12 & $22: 34: 21$ & 85.9 & 276.4 & 7.0 & -0.5 & 32.2 & 0 \\
\hline 2011/09/15 & $22: 46: 49$ & 86.1 & 273.9 & 15.4 & -6.8 & 36.2 & 3 \\
\hline 2011/09/15 & $22: 46: 52$ & 82.7 & 273.9 & 15.4 & -6.8 & 36.2 & 3 \\
\hline $2011 / 09 / 22$ & $22: 42: 29$ & 12.3 & 273.3 & -8.8 & -0.9 & 40.0 & 3 \\
\hline $2011 / 09 / 22$ & $22: 42: 33$ & 13.8 & 273.3 & -8.8 & -0.9 & 40.0 & 3 \\
\hline $2011 / 09 / 24$ & $21: 42: 36$ & 40.2 & 275.4 & 1.3 & -5.1 & 35.3 & 2 \\
\hline $2011 / 09 / 28$ & $21: 25: 57$ & 37.3 & 275.4 & -1.6 & 4.3 & 25.5 & 2 \\
\hline 2011/10/06 & 12:38:34 & 45.1 & 273.2 & -5.8 & 7.0 & 39.1 & 3 \\
\hline 2011/10/08 & $21: 34: 17$ & 28.4 & 273.7 & 3.1 & -4.5 & 30.0 & 2 \\
\hline $2011 / 10 / 23$ & 11:03:15 & 0 & 272.5 & -13.2 & 1.9 & 37.2 & 1 \\
\hline 2011/11/01 & $21: 34: 17$ & 25.8 & 271.8 & -6.7 & 2.1 & 30.0 & 1 \\
\hline 2012/01/18 & 21:59:18 & unknown & 238.1 & 1.2 & 1.8 & 43.5 & 2 \\
\hline $2012 / 01 / 26$ & $21: 25: 16$ & unknown & 252.4 & 9.8 & -6.8 & 27.8 & 3 \\
\hline
\end{tabular}

Table 8.3: Test images used for assessing accuracy of SVM/SLIC classification

\begin{tabular}{ccc}
\hline Date (GMT) & Time (GMT) & Beam Mode \\
\hline $2010 / 09 / 11$ & $23: 50: 36$ & FQ14 \\
$2012 / 06 / 14$ & $13: 28: 38$ & FQ6W \\
$2012 / 06 / 14$ & $21: 42: 37$ & FQ15W \\
$2012 / 10 / 29$ & $21: 46: 43$ & FQ17W \\
$2013 / 03 / 26$ & $11: 36: 17$ & FQ13W \\
$2013 / 04 / 08$ & $21: 50: 38$ & FQ19W \\
$2013 / 06 / 17$ & $11: 16: 08$ & FQ4W \\
$2013 / 06 / 29$ & $21: 58: 59$ & FQ24 \\
$2013 / 07 / 02$ & $22: 12: 26$ & FQ22 \\
\hline
\end{tabular}




\section{Appendix C}

\subsection{ANOVA Separability of SAR Variables}

Table 9.1: Measured ANOVA p-values for all 123 SAR variables

\begin{tabular}{|c|c|}
\hline SAR Variable & ANOVA p-value \\
\hline reflectivity_22ov11 & $<0.00001$ \\
\hline reflectivity_11ov22 & $<0.00001$ \\
\hline beta & $<0.00001$ \\
\hline entropy_shannon_P_norm & $<0.00001$ \\
\hline reflectivity_22ov12 & $<0.00001$ \\
\hline beta1 & $<0.00001$ \\
\hline delta & $<0.00001$ \\
\hline T12_real & $<0.00001$ \\
\hline A12 & $<0.00001$ \\
\hline A21 & $<0.00001$ \\
\hline entropy_shannon & $<0.00001$ \\
\hline entropy_shannon_norm & $<0.00001$ \\
\hline Krogager_Kd & $<0.00001$ \\
\hline reflectivity_12ov22 & $<0.00001$ \\
\hline T23_mod & $<0.00001$ \\
\hline derd & $<0.00001$ \\
\hline derd_norm & $<0.00001$ \\
\hline $\mathrm{I} 12$ & $<0.00001$ \\
\hline I21 & $<0.00001$ \\
\hline 12 & $<0.00001$ \\
\hline entropy_shannon_P & $<0.00001$ \\
\hline beta3 & $<0.00001$ \\
\hline Krogager_Kh & $<0.00001$ \\
\hline T33_mod & $<0.00001$ \\
\hline $\mathrm{C} 22$ & $<0.00001$ \\
\hline Freeman_Vol & $<0.00001$ \\
\hline combination_1mHA & $<0.00001$ \\
\hline T33 & $<0.00001$ \\
\hline VanZyl3_Vol & $<0.00001$ \\
\hline 13 & $<0.00001$ \\
\hline beta2 & $<0.00001$ \\
\hline combination_H1mA & $<0.00001$ \\
\hline $\mathrm{T} 22$ & $<0.00001$ \\
\hline T22_mod & $<0.00001$ \\
\hline Neumann_delta_pha & $<0.00001$ \\
\hline delta1 & $<0.00001$ \\
\hline VanZyl3_Dbl & $<0.00001$ \\
\hline serd & $<0.00001$ \\
\hline serd_norm & $<0.00001$ \\
\hline
\end{tabular}




\begin{tabular}{|c|c|}
\hline SAR Variable & ANOVA p-value \\
\hline entropy & $<0.00001$ \\
\hline TSVM_alpha_s3 & $<0.00001$ \\
\hline rvi & $<0.00001$ \\
\hline p3 & $<0.00001$ \\
\hline polarisation_fraction & $<0.00001$ \\
\hline TSVM_alpha_s1 & $<0.00001$ \\
\hline T13_mod & $<0.00001$ \\
\hline reflectivity_12ov11 & $<0.00001$ \\
\hline pedestal & $<0.00001$ \\
\hline anisotropy & $<0.00001$ \\
\hline $\mathrm{p} 1$ & $<0.00001$ \\
\hline reflectivity_11ov12 & $<0.00001$ \\
\hline TSVM_alpha_s2 & $<0.00001$ \\
\hline entropy_shannon_I_norm & $<0.00001$ \\
\hline entropy_shannon_I & $<0.00001$ \\
\hline anisotropy_lueneburg & $<0.00001$ \\
\hline anisotropy12 & $<0.00001$ \\
\hline p2 & $<0.00001$ \\
\hline T12_mod & $<0.00001$ \\
\hline A11 & $<0.00001$ \\
\hline alpha2 & $<0.00001$ \\
\hline s2_span & $<0.00001$ \\
\hline $\mathrm{I} 11$ & $<0.00001$ \\
\hline C11 & $<0.00001$ \\
\hline Neumann_delta_mod & $<0.00001$ \\
\hline wishart_H_alpha_class_3 & $<0.00001$ \\
\hline $\mathrm{A} 22$ & $<0.00001$ \\
\hline Krogager_Ks & $<0.00001$ \\
\hline alpha1 & $<0.00001$ \\
\hline $\mathrm{I} 22$ & $<0.00001$ \\
\hline C33 & $<0.00001$ \\
\hline alpha3 & $<0.00001$ \\
\hline 11 & $<0.00001$ \\
\hline T12_imag & $<0.00001$ \\
\hline C13_imag & $<0.00001$ \\
\hline combination_1mH1mA & $<0.00001$ \\
\hline T11_mod & $<0.00001$ \\
\hline $\mathrm{T} 11$ & $<0.00001$ \\
\hline alpha & $<0.00001$ \\
\hline VanZyl3_Odd & $<0.00001$ \\
\hline lambda & $<0.00001$ \\
\hline C13_real & $<0.00001$ \\
\hline asymetry & $<0.00001$ \\
\hline Freeman_Odd & $<0.00001$ \\
\hline TSVM_alpha_s & $<0.00001$ \\
\hline combination_HA & $<0.00001$ \\
\hline TSVM_phi_s & $<0.00001$ \\
\hline
\end{tabular}




\begin{tabular}{|c|c|}
\hline SAR Variable & ANOVA p-value \\
\hline delta3 & 0.00003 \\
\hline TSVM_psi & 0.00005 \\
\hline TSVM_psi1 & 0.00008 \\
\hline TSVM_phi_s3 & 0.00008 \\
\hline delta2 & 0.00009 \\
\hline TSVM_phi_s1 & 0.0001 \\
\hline TSVM_phi_s2 & 0.00016 \\
\hline C12_real & 0.00017 \\
\hline T13_real & 0.00037 \\
\hline TSVM_psi2 & 0.00165 \\
\hline Freeman_Dbl & 0.00213 \\
\hline TSVM_psi3 & 0.00794 \\
\hline s12_pha & 0.04091 \\
\hline T23_imag & 0.04277 \\
\hline wishart_H_A_alpha_class_3 & 0.06000 \\
\hline Neumann_psi & 0.06268 \\
\hline s21_pha & 0.09639 \\
\hline T23_real & 0.09763 \\
\hline TSVM_tau_m2 & 0.12726 \\
\hline s22 & 0.13372 \\
\hline s21 & 0.22407 \\
\hline TSVM_tau_m1 & 0.27272 \\
\hline C23_real & 0.39087 \\
\hline TSVM_tau_m3 & 0.42681 \\
\hline gamma2 & 0.68883 \\
\hline gamma3 & 0.70763 \\
\hline s11_pha & 0.77686 \\
\hline gamma & 0.82042 \\
\hline TSVM_tau_m & 0.82902 \\
\hline gamma1 & 0.84023 \\
\hline s12 & 0.84886 \\
\hline s22_pha & 0.88986 \\
\hline s11 & 0.95775 \\
\hline C12_imag & 0.99908 \\
\hline C23_imag & 0.99947 \\
\hline T13_imag & 0.99988 \\
\hline
\end{tabular}




\section{Appendix D}

\subsection{Assessment of SAR Variables for Separating each Ocean Cover Based Upon THSD/G-H}

Table 10.1: SAR variables maximum base-level difference of means scores for separating water from other ocean covers.

\begin{tabular}{|c|c|c|c|}
\hline SAR Variable & $\begin{array}{c}\text { Maximum Base-Level } \\
\text { Difference of Means } \\
\text { Score } \\
\end{array}$ & $\begin{array}{c}\text { THSD p-value } \\
\text { (corrected with FDR) }\end{array}$ & $\begin{array}{c}\text { G-H p-value } \\
\text { (corrected with FDR) }\end{array}$ \\
\hline delta1 & 0.53725 & $<0.00001$ & $<0.00001$ \\
\hline Neumann_delta_pha & 0.53679 & $<0.00001$ & $<0.00001$ \\
\hline delta & 0.51270 & $<0.00001$ & $<0.00001$ \\
\hline reflectivity_11ov22 & 0.50779 & $<0.00001$ & $<0.00001$ \\
\hline reflectivity_22ov11 & 0.49655 & $<0.00001$ & $<0.00001$ \\
\hline beta & 0.49289 & $<0.00001$ & $<0.00001$ \\
\hline beta1 & 0.49106 & $<0.00001$ & $<0.00001$ \\
\hline entropy_shannon_P_norm & 0.34752 & $<0.00001$ & $<0.00001$ \\
\hline TSVM_alpha_s1 & 0.33882 & $<0.00001$ & 0.00271 \\
\hline alpha2 & 0.33490 & $<0.00001$ & 0.00013 \\
\hline T12_real & 0.27845 & $<0.00001$ & 0.00079 \\
\hline $\mathrm{p} 2$ & 0.26466 & $<0.00001$ & $<0.00001$ \\
\hline entropy & 0.25907 & $<0.00001$ & $<0.00001$ \\
\hline anisotropy12 & 0.25658 & 0.00053 & 0.01067 \\
\hline delta2 & 0.25517 & 0.0018 & $<0.00001$ \\
\hline reflectivity_12ov22 & 0.25063 & $<0.00001$ & $<0.00001$ \\
\hline reflectivity_22ov12 & 0.24313 & $<0.00001$ & 0.01905 \\
\hline entropy_shannon_P & 0.24234 & $<0.00001$ & $<0.00001$ \\
\hline $\mathrm{p} 1$ & 0.23888 & 0.00096 & 0.01796 \\
\hline TSVM_phi_S & 0.23790 & $<0.00001$ & $<0.00001$ \\
\hline TSVM_phi_s2 & 0.23765 & 0.02679 & 0.0034 \\
\hline anisotropy_lueneburg & 0.22920 & $<0.00001$ & $<0.00001$ \\
\hline combination_1mH1mA & 0.22557 & 0.00047 & 0.00018 \\
\hline TSVM_phi_s1 & 0.22415 & 0.00006 & 0.00001 \\
\hline alpha1 & 0.20890 & 0.00023 & 0.06386 \\
\hline combination_H1mA & 0.17731 & $<0.00001$ & 0.00001 \\
\hline p3 & 0.17615 & $<0.00001$ & $<0.00001$ \\
\hline polarisation_fraction & 0.17534 & 0.02221 & 0.07918 \\
\hline rvi & 0.17441 & $<0.00001$ & $<0.00001$ \\
\hline asymetry & 0.17298 & 0.00119 & $<0.00001$ \\
\hline serd_norm & 0.16923 & 0.03137 & 0.07401 \\
\hline serd & 0.16863 & 0.03249 & 0.07444 \\
\hline Neumann_delta_mod & 0.16204 & $<0.00001$ & $<0.00001$ \\
\hline pedestal & 0.15771 & $<0.00001$ & $<0.00001$ \\
\hline delta3 & 0.15423 & 0.24497 & 0.00287 \\
\hline
\end{tabular}




\begin{tabular}{|c|c|c|c|}
\hline SAR Variable & $\begin{array}{c}\text { Maximum Base-Level } \\
\text { Difference of Means } \\
\text { Score } \\
\end{array}$ & $\begin{array}{c}\text { THSD p-value } \\
\text { (corrected with FDR) }\end{array}$ & $\begin{array}{c}\text { G-H p-value } \\
\text { (corrected with FDR) }\end{array}$ \\
\hline combination_HA & 0.14702 & $<0.00001$ & 0.00022 \\
\hline TSVM_psi & 0.13639 & 0.00002 & 0.57941 \\
\hline TSVM_psi1 & 0.13119 & 0.00002 & 0.5294 \\
\hline reflectivity_12ov11 & 0.09802 & $<0.00001$ & $<0.00001$ \\
\hline A22 & 0.09482 & 0.00001 & 0.00017 \\
\hline combination_1mHA & 0.08894 & 0.29442 & 0.99016 \\
\hline reflectivity_11ov12 & 0.08480 & 0.20616 & 0.97373 \\
\hline entropy_shannon_I_norm & 0.08097 & $<0.00001$ & $<0.00001$ \\
\hline entropy_shannon_I & 0.07840 & $<0.00001$ & 0.00022 \\
\hline Krogager_Kh & 0.06940 & $<0.00001$ & $<0.00001$ \\
\hline TSVM_alpha_s2 & 0.06876 & 0.94864 & 0.66087 \\
\hline alpha & 0.06745 & $<0.00001$ & 0.00004 \\
\hline T12_mod & 0.06633 & 0.11193 & 0.51524 \\
\hline I22 & 0.06452 & $<0.00001$ & $<0.00001$ \\
\hline Krogager_Ks & 0.06374 & $<0.00001$ & 0.00002 \\
\hline C33 & 0.06205 & 0.00002 & $<0.00001$ \\
\hline Freeman_Odd & 0.05698 & 0.001 & 0.00015 \\
\hline TSVM_tau_m3 & 0.05205 & 0.70115 & 0.00944 \\
\hline TSVM_alpha_s3 & 0.05197 & 1 & 1 \\
\hline anisotropy & 0.04823 & 1 & 1 \\
\hline lambda & 0.04782 & 0.00006 & $<0.00001$ \\
\hline T12_imag & 0.04660 & 1 & 0.00034 \\
\hline C13_imag & 0.04565 & $<0.00001$ & $<0.00001$ \\
\hline VanZyl3_Odd & 0.04537 & 0.00004 & $<0.00001$ \\
\hline C13_real & 0.04069 & 0.00008 & $<0.00001$ \\
\hline A12 & 0.04024 & $<0.00001$ & $<0.00001$ \\
\hline TSVM_psi3 & 0.04017 & 0.98519 & 1 \\
\hline A21 & 0.03838 & $<0.00001$ & $<0.00001$ \\
\hline TSVM_phi_s3 & 0.03751 & 1 & 0.63227 \\
\hline 11 & 0.03645 & $<0.00001$ & $<0.00001$ \\
\hline VanZyl3_Dbl & 0.03495 & $<0.00001$ & $<0.00001$ \\
\hline T11_mod & 0.03465 & $<0.00001$ & $<0.00001$ \\
\hline $\mathrm{T} 11$ & 0.03423 & $<0.00001$ & $<0.00001$ \\
\hline entropy_shannon_norm & 0.03130 & $<0.00001$ & $<0.00001$ \\
\hline beta3 & 0.03109 & 1 & 1 \\
\hline 12 & 0.02815 & $<0.00001$ & $<0.00001$ \\
\hline s12_pha & 0.02711 & 0.99871 & 0.85243 \\
\hline gamma2 & 0.02669 & 0.84831 & 0.00632 \\
\hline T23_mod & 0.02546 & $<0.00001$ & $<0.00001$ \\
\hline beta2 & 0.02543 & $<0.00001$ & $<0.00001$ \\
\hline T33 & 0.02450 & $<0.00001$ & $<0.00001$ \\
\hline $\mathrm{C} 22$ & 0.02434 & $<0.00001$ & $<0.00001$ \\
\hline 13 & 0.02358 & $<0.00001$ & $<0.00001$ \\
\hline T33_mod & 0.02319 & $<0.00001$ & $<0.00001$ \\
\hline Freeman_Vol & 0.02294 & $<0.00001$ & $<0.00001$ \\
\hline I21 & 0.02225 & $<0.00001$ & $<0.00001$ \\
\hline I12 & 0.02180 & $<0.00001$ & $<0.00001$ \\
\hline
\end{tabular}




\begin{tabular}{|c|c|c|c|}
\hline SAR Variable & $\begin{array}{c}\text { Maximum Base-Level } \\
\text { Difference of Means } \\
\text { Score }\end{array}$ & $\begin{array}{c}\text { THSD p-value } \\
\text { (corrected with FDR) }\end{array}$ & $\begin{array}{c}\text { G-H p-value } \\
\text { (corrected with FDR) }\end{array}$ \\
\hline s2_span & 0.02128 & $<0.00001$ & $<0.00001$ \\
\hline derd & 0.01911 & 1 & 1 \\
\hline alpha3 & 0.01844 & 1 & 1 \\
\hline derd_norm & 0.01816 & 1 & 1 \\
\hline T23_imag & 0.01788 & 1 & 0.6946 \\
\hline C23_real & 0.01573 & 0.66442 & 0.42715 \\
\hline entropy_shannon & 0.01487 & $<0.00001$ & $<0.00001$ \\
\hline TSVM_psi2 & 0.01437 & 1 & 1 \\
\hline T13_mod & 0.01310 & $<0.00001$ & $<0.00001$ \\
\hline Krogager_Kd & 0.01107 & $<0.00001$ & $<0.00001$ \\
\hline A11 & 0.01079 & $<0.00001$ & $<0.00001$ \\
\hline Freeman_Dbl & 0.01067 & 0.4024 & 0.73234 \\
\hline T13_real & 0.01056 & 0.05715 & 0.01055 \\
\hline VanZyl3_Vol & 0.01044 & $<0.00001$ & $<0.00001$ \\
\hline TSVM_tau_m2 & 0.00957 & 1 & 1 \\
\hline I11 & 0.00928 & $<0.00001$ & $<0.00001$ \\
\hline T23_real & 0.00901 & 0.9743 & 0.6946 \\
\hline wishart_H_alpha_class_3 & 0.00884 & $<0.00001$ & $<0.00001$ \\
\hline TSVM_tau_m1 & 0.00867 & 1 & 0.84671 \\
\hline wishart_H_A_alpha_class_3 & 0.00861 & 0.58627 & 0.86831 \\
\hline C11 & 0.00812 & $<0.00001$ & $<0.00001$ \\
\hline s22 & 0.00678 & 0.52533 & 0.23006 \\
\hline TSVM_alpha_s & 0.00423 & 0.0112 & 0.06829 \\
\hline s22_pha & 0.00383 & 1 & 1 \\
\hline s21_pha & 0.00375 & 1 & 0.31513 \\
\hline gamma3 & 0.00370 & 1 & 1 \\
\hline s21 & 0.00361 & 0.80924 & 0.5294 \\
\hline gamma & 0.00310 & 1 & 1 \\
\hline s11_pha & 0.00303 & 1 & 1 \\
\hline gamma1 & 0.00294 & 1 & 1 \\
\hline Neumann_psi & 0.00267 & 0.97109 & 0.66087 \\
\hline C12_real & 0.00173 & 0.09526 & 0.01817 \\
\hline C23_imag & 0.00147 & 1 & 1 \\
\hline $\mathrm{T} 22$ & 0.00120 & $<0.00001$ & $<0.00001$ \\
\hline s11 & 0.00108 & 1 & 1 \\
\hline C12_imag & 0.00078 & 1 & 1 \\
\hline T22_mod & 0.00065 & $<0.00001$ & $<0.00001$ \\
\hline TSVM_tau_m & 0.00057 & 1 & 0.40309 \\
\hline s12 & 0.00056 & 1 & 0.86831 \\
\hline T13_imag & 0.00012 & 1 & 1 \\
\hline
\end{tabular}


Table 10.2: SAR variables maximum base-level difference of means scores for separating FYI from other ocean covers.

\begin{tabular}{|c|c|c|c|}
\hline SAR Variable & $\begin{array}{l}\text { Maximum Base- } \\
\text { Level Difference of } \\
\text { Means Score }\end{array}$ & $\begin{array}{c}\text { THSD p-value } \\
\text { (corrected with } \\
\text { FDR) }\end{array}$ & $\begin{array}{c}\text { G-H p-value } \\
\text { (corrected with } \\
\text { FDR) }\end{array}$ \\
\hline combination_HA & 0.12147 & $<0.00001$ & 0.0003 \\
\hline beta1 & 0.12090 & $<0.00001$ & $<0.00001$ \\
\hline combination_H1mA & 0.11885 & $<0.00001$ & 0.00286 \\
\hline Freeman_Dbl & 0.11221 & 0.00645 & 0.00125 \\
\hline A22 & 0.11048 & $<0.00001$ & $<0.00001$ \\
\hline reflectivity_12ov11 & 0.09802 & $<0.00001$ & 0.00296 \\
\hline combination_1mHA & 0.08894 & 0.32839 & 1 \\
\hline reflectivity_11ov12 & 0.08480 & 0.23057 & 1 \\
\hline T12_mod & 0.08443 & $<0.00001$ & $<0.00001$ \\
\hline reflectivity_22ov12 & 0.08393 & 0.02594 & 0.6182 \\
\hline polarisation_fraction & 0.08306 & 0.51147 & 0.8101 \\
\hline rvi & 0.08297 & $<0.00001$ & 0.00259 \\
\hline reflectivity_12ov22 & 0.08274 & $<0.00001$ & 0.00643 \\
\hline entropy_shannon_I_norm & 0.08097 & $<0.00001$ & $<0.00001$ \\
\hline p3 & 0.08084 & $<0.00001$ & 0.00265 \\
\hline entropy_shannon_I & 0.07840 & $<0.00001$ & 0.00007 \\
\hline entropy_shannon_P & 0.07520 & $<0.00001$ & 0.03172 \\
\hline serd & 0.07221 & 0.7058 & 0.90193 \\
\hline C33 & 0.07112 & $<0.00001$ & $<0.00001$ \\
\hline serd_norm & 0.07045 & 0.73119 & 0.91875 \\
\hline Krogager_Kh & 0.06940 & $<0.00001$ & $<0.00001$ \\
\hline TSVM_alpha_s2 & 0.06876 & 1 & 0.74639 \\
\hline $\mathrm{I} 22$ & 0.06542 & $<0.00001$ & $<0.00001$ \\
\hline Krogager_Ks & 0.06374 & $<0.00001$ & $<0.00001$ \\
\hline pedestal & 0.06110 & $<0.00001$ & 0.00259 \\
\hline wishart_H_A_alpha_class_3 & 0.06032 & 0.63571 & 0.93592 \\
\hline reflectivity_11ov22 & 0.05937 & $<0.00001$ & $<0.00001$ \\
\hline Freeman_Odd & 0.05698 & 0.00003 & $<0.00001$ \\
\hline entropy & 0.05550 & 0.00007 & 0.01549 \\
\hline TSVM_alpha_s3 & 0.05197 & 1 & 1 \\
\hline anisotropy & 0.04823 & 1 & 1 \\
\hline lambda & 0.04782 & $<0.00001$ & $<0.00001$ \\
\hline T12_imag & 0.04660 & 1 & 0.00046 \\
\hline C13_imag & 0.04565 & 0.00006 & $<0.00001$ \\
\hline VanZyl3_Odd & 0.04537 & $<0.00001$ & $<0.00001$ \\
\hline reflectivity_22ov11 & 0.04290 & 0.05765 & 0.00223 \\
\hline TSVM_phi_s1 & 0.04256 & 0.05376 & 0.00156 \\
\hline delta3 & 0.04211 & 1 & 1 \\
\hline C13_real & 0.04069 & $<0.00001$ & $<0.00001$ \\
\hline A12 & 0.04024 & $<0.00001$ & $<0.00001$ \\
\hline A21 & 0.03838 & $<0.00001$ & $<0.00001$ \\
\hline 11 & 0.03645 & $<0.00001$ & $<0.00001$ \\
\hline VanZyl3_Dbl & 0.03495 & $<0.00001$ & $<0.00001$ \\
\hline $\mathrm{p} 1$ & 0.03466 & 1 & 1 \\
\hline
\end{tabular}




\begin{tabular}{|c|c|c|c|}
\hline SAR Variable & $\begin{array}{l}\text { Maximum Base- } \\
\text { Level Difference of } \\
\text { Means Score }\end{array}$ & $\begin{array}{c}\text { THSD p-value } \\
\text { (corrected with } \\
\text { FDR) }\end{array}$ & $\begin{array}{c}\text { G-H p-value } \\
\text { (corrected with } \\
\text { FDR) }\end{array}$ \\
\hline T11_mod & 0.03465 & $<0.00001$ & $<0.00001$ \\
\hline combination_1mH1mA & 0.03463 & 0.35621 & 0.34322 \\
\hline $\mathrm{T} 11$ & 0.03423 & $<0.00001$ & $<0.00001$ \\
\hline Neumann_delta_mod & 0.03149 & 0.00045 & 0.05536 \\
\hline entropy_shannon_norm & 0.03130 & $<0.00001$ & 0.00003 \\
\hline beta3 & 0.03109 & 1 & 1 \\
\hline alpha & 0.02989 & 0.00028 & 0.03468 \\
\hline delta & 0.02934 & $<0.00001$ & 0.00003 \\
\hline 12 & 0.02815 & $<0.00001$ & $<0.00001$ \\
\hline gamma1 & 0.02683 & 1 & 1 \\
\hline TSVM_tau_m2 & 0.02559 & 0.52362 & 0.09587 \\
\hline T12_real & 0.02549 & $<0.00001$ & 0.00223 \\
\hline T23_mod & 0.02546 & $<0.00001$ & $<0.00001$ \\
\hline beta2 & 0.02543 & $<0.00001$ & 0.00002 \\
\hline Т33 & 0.02450 & $<0.00001$ & $<0.00001$ \\
\hline $\mathrm{C} 22$ & 0.02434 & $<0.00001$ & $<0.00001$ \\
\hline gamma3 & 0.02375 & 1 & 0.68182 \\
\hline 13 & 0.02358 & $<0.00001$ & $<0.00001$ \\
\hline T33_mod & 0.02319 & $<0.00001$ & $<0.00001$ \\
\hline Freeman_Vol & 0.02294 & $<0.00001$ & $<0.00001$ \\
\hline $\mathrm{I} 21$ & 0.02225 & $<0.00001$ & $<0.00001$ \\
\hline $\mathrm{I} 12$ & 0.02180 & $<0.00001$ & $<0.00001$ \\
\hline asymetry & 0.02132 & 0.80643 & 0.93592 \\
\hline s2_span & 0.02128 & $<0.00001$ & $<0.00001$ \\
\hline entropy_shannon_P_norm & 0.02061 & $<0.00001$ & 0.00016 \\
\hline s21 & 0.01986 & 1 & 1 \\
\hline derd & 0.01911 & 1 & 1 \\
\hline alpha3 & 0.01844 & 1 & 1 \\
\hline Neumann_psi & 0.01832 & 0.30991 & 0.04744 \\
\hline derd_norm & 0.01816 & 1 & 1 \\
\hline gamma & 0.01782 & 1 & 1 \\
\hline TSVM_alpha_s1 & 0.01742 & 0.13689 & 0.16794 \\
\hline anisotropy_lueneburg & 0.01599 & 0.00296 & 0.02157 \\
\hline T23_imag & 0.01566 & 0.34965 & 0.01967 \\
\hline TSVM_tau_m3 & 0.01545 & 0.74144 & 0.01089 \\
\hline entropy_shannon & 0.01487 & $<0.00001$ & $<0.00001$ \\
\hline TSVM_psi2 & 0.01437 & 1 & 1 \\
\hline s12_pha & 0.01408 & 1 & 0.93582 \\
\hline T13_mod & 0.01310 & $<0.00001$ & $<0.00001$ \\
\hline delta2 & 0.01280 & 1 & 1 \\
\hline alpha2 & 0.01132 & $<0.00001$ & 0.00021 \\
\hline Krogager_Kd & 0.01107 & $<0.00001$ & $<0.00001$ \\
\hline s12 & 0.01085 & 1 & 0.93592 \\
\hline A11 & 0.01079 & $<0.00001$ & $<0.00001$ \\
\hline VanZyl3_Vol & 0.01044 & $<0.00001$ & $<0.00001$ \\
\hline TSVM_phi_s & 0.00959 & 0.0112 & 0.00265 \\
\hline $\mathrm{I} 11$ & 0.00928 & $<0.00001$ & $<0.00001$ \\
\hline
\end{tabular}




\begin{tabular}{|c|c|c|c|}
\hline SAR Variable & $\begin{array}{l}\text { Maximum Base- } \\
\text { Level Difference of } \\
\text { Means Score }\end{array}$ & $\begin{array}{l}\text { THSD p-value } \\
\text { (corrected with } \\
\text { FDR) }\end{array}$ & $\begin{array}{c}\text { G-H p-value } \\
\text { (corrected with } \\
\text { FDR) }\end{array}$ \\
\hline wishart_H_alpha_class_3 & 0.00884 & $<0.00001$ & $<0.00001$ \\
\hline TSVM tau m1 & 0.00876 & 1 & 0.5841 \\
\hline C11 & 0.00812 & $<0.00001$ & $<0.00001$ \\
\hline Neumann_delta_pha & 0.00794 & 1 & 1 \\
\hline s22_pha & 0.00640 & 1 & 1 \\
\hline gamma2 & 0.00631 & 1 & 0.10523 \\
\hline delta1 & 0.00589 & $<0.00001$ & 0.00002 \\
\hline T23_real & 0.00561 & 0.66527 & 0.26275 \\
\hline Neumann_tau & 0.00561 & $<0.00001$ & $<0.00001$ \\
\hline TSVM_alpha_s & 0.00507 & 0.02399 & 0.26417 \\
\hline TSVM_phi_s3 & 0.00505 & 1 & 1 \\
\hline anisotropy12 & 0.00468 & 1 & 1 \\
\hline s11_pha & 0.00440 & 1 & 1 \\
\hline s21_pha & 0.00375 & 1 & 1 \\
\hline p2 & 0.00363 & 0.00022 & 0.01003 \\
\hline C23_imag & 0.00307 & 1 & 1 \\
\hline TSVM_psi1 & 0.00267 & 0.04329 & 0.86841 \\
\hline alpha1 & 0.00223 & 0.03316 & 0.04227 \\
\hline C12_real & 0.00192 & 0.11319 & 0.00993 \\
\hline T13_real & 0.00151 & 0.17092 & 0.014 \\
\hline s22 & 0.00143 & 1 & 0.86841 \\
\hline $\mathrm{T} 22$ & 0.00120 & $<0.00001$ & $<0.00001$ \\
\hline T13_imag & 0.00110 & 1 & 1 \\
\hline s11 & 0.00108 & 1 & 1 \\
\hline C23_real & 0.00091 & 1 & 0.93592 \\
\hline TSVM_phi_s2 & 0.00081 & 1 & 1 \\
\hline C12_imag & 0.00078 & 1 & 1 \\
\hline T22_mod & 0.00065 & $<0.00001$ & $<0.00001$ \\
\hline TSVM_tau_m & 0.00057 & 1 & 0.6182 \\
\hline TSVM_psi3 & 0.00000 & 1 & 1 \\
\hline TSVM psi & 0.00000 & 0.0112 & 0.80548 \\
\hline
\end{tabular}


Table 10.3: SAR variables maximum base-level difference of means scores for separating marine ice from other ocean covers.

\begin{tabular}{|c|c|c|c|}
\hline SAR Variable & $\begin{array}{l}\text { Maximum Base- } \\
\text { Level Difference of } \\
\text { Means Score }\end{array}$ & $\begin{array}{c}\text { THSD p-value } \\
\text { (corrected with FDR) }\end{array}$ & $\begin{array}{c}\text { G-H p-value (corrected } \\
\text { with FDR) }\end{array}$ \\
\hline beta3 & 0.15473 & $<0.00001$ & $<0.00001$ \\
\hline Krogager_Kh & 0.14554 & $<0.00001$ & $<0.00001$ \\
\hline TSVM_alpha_s3 & 0.14216 & 0.00008 & $<0.00001$ \\
\hline entropy_shannon_norm & 0.14153 & $<0.00001$ & $<0.00001$ \\
\hline derd_norm & 0.13385 & $<0.00001$ & $<0.00001$ \\
\hline derd & 0.13007 & 0.00002 & $<0.00001$ \\
\hline Krogager_Kd & 0.12823 & 0.00188 & 0.00002 \\
\hline T23_mod & 0.11864 & 0.09951 & $<0.00001$ \\
\hline entropy_shannon_I_norm & 0.11856 & 0.0004 & 0.0066 \\
\hline A12 & 0.11690 & 0.00014 & $<0.00001$ \\
\hline A21 & 0.11295 & 0.00014 & $<0.00001$ \\
\hline combination_H1mA & 0.10951 & $<0.00001$ & $<0.00001$ \\
\hline wishart_H_alpha_class_3 & 0.10807 & 0.24795 & 0.01027 \\
\hline reflectivity_12ov11 & 0.10717 & 0.00003 & 0.00545 \\
\hline 13 & 0.10688 & 0.17436 & $<0.00001$ \\
\hline entropy_shannon & 0.10680 & $<0.00001$ & 0.00013 \\
\hline 12 & 0.10656 & 0.10344 & $<0.00001$ \\
\hline T33 & 0.10415 & 0.17436 & $<0.00001$ \\
\hline T33_mod & 0.10388 & 0.17436 & $<0.00001$ \\
\hline $\mathrm{C} 22$ & 0.10111 & 0.17436 & $<0.00001$ \\
\hline $\mathrm{I} 12$ & 0.10071 & 0.17436 & $<0.00001$ \\
\hline $\mathrm{I} 21$ & 0.10048 & 0.16797 & $<0.00001$ \\
\hline Freeman_Vol & 0.10028 & 0.19482 & $<0.00001$ \\
\hline $\mathrm{T} 22$ & 0.09861 & 0.25808 & 0.00009 \\
\hline T22_mod & 0.09742 & 0.25808 & 0.00009 \\
\hline anisotropy & 0.09425 & 0.00677 & 0.00209 \\
\hline VanZyl3_Vol & 0.08913 & 0.3682 & $<0.00001$ \\
\hline entropy_shannon_I & 0.08532 & 0.00002 & 0.00669 \\
\hline polarisation_fraction & 0.08306 & $<0.00001$ & $<0.00001$ \\
\hline rvi & 0.08297 & $<0.00001$ & $<0.00001$ \\
\hline reflectivity_12ov22 & 0.08274 & $<0.00001$ & $<0.00001$ \\
\hline T13_mod & 0.08238 & 0.09962 & $<0.00001$ \\
\hline p3 & 0.08084 & $<0.00001$ & $<0.00001$ \\
\hline Krogager_Ks & 0.07974 & 0.00906 & 0.00923 \\
\hline combination_1mHA & 0.07967 & 0.00031 & $<0.00001$ \\
\hline TSVM_alpha_s2 & 0.07964 & 0.00226 & 0.00957 \\
\hline combination_1mH1mA & 0.07780 & 0.00012 & 0.00035 \\
\hline serd & 0.07221 & $<0.00001$ & $<0.00001$ \\
\hline serd_norm & 0.07045 & $<0.00001$ & $<0.00001$ \\
\hline C11 & 0.06974 & 0.151 & 0.00013 \\
\hline s2_span & 0.06764 & 0.17436 & 0.00958 \\
\hline 11 & 0.06692 & 0.18088 & 0.02154 \\
\hline T11 & 0.06544 & 0.17761 & 0.01853 \\
\hline T11_mod & 0.06534 & 0.17436 & 0.0172 \\
\hline C33 & 0.06205 & 0.16256 & 0.01444 \\
\hline
\end{tabular}




\begin{tabular}{|c|c|c|c|}
\hline SAR Variable & $\begin{array}{l}\text { Maximum Base- } \\
\text { Level Difference of } \\
\text { Means Score }\end{array}$ & $\begin{array}{c}\text { THSD p-value } \\
\text { (corrected with FDR) }\end{array}$ & $\begin{array}{c}\text { G-H p-value (corrected } \\
\text { with FDR) }\end{array}$ \\
\hline entropy_shannon_P & 0.06200 & $<0.00001$ & 0.00018 \\
\hline pedestal & 0.06110 & 0.00004 & $<0.00001$ \\
\hline C13_real & 0.05957 & 0.19482 & 0.0206 \\
\hline lambda & 0.05789 & 0.18088 & 0.01788 \\
\hline VanZyl3_Odd & 0.05700 & 0.17436 & 0.01668 \\
\hline A22 & 0.05672 & 0.0033 & 0.00235 \\
\hline entropy & 0.05550 & $<0.00001$ & $<0.00001$ \\
\hline wishart_H_A_alpha_class_3 & 0.05364 & 0.25808 & 0.22037 \\
\hline $\mathrm{A} 11$ & 0.05169 & 0.00349 & 0.00034 \\
\hline T12_mod & 0.05074 & 0.43589 & 0.00098 \\
\hline VanZyl3_Dbl & 0.05031 & 0.32382 & 0.01465 \\
\hline I11 & 0.05023 & 0.12524 & 0.00007 \\
\hline reflectivity_11ov12 & 0.04969 & 0.00482 & $<0.00001$ \\
\hline I22 & 0.04959 & 0.151 & 0.00458 \\
\hline delta1 & 0.04728 & $<0.00001$ & $<0.00001$ \\
\hline Neumann_delta_pha & 0.04420 & 0.82862 & 0.58961 \\
\hline reflectivity_22ov12 & 0.04400 & 0.00236 & $<0.00001$ \\
\hline s22 & 0.04390 & 0.49738 & 0.21742 \\
\hline alpha3 & 0.04361 & $<0.00001$ & $<0.00001$ \\
\hline Freeman_Odd & 0.04279 & 0.17436 & 0.01292 \\
\hline $\mathrm{p} 1$ & 0.03466 & $<0.00001$ & $<0.00001$ \\
\hline delta3 & 0.03213 & 0.26005 & 0.09656 \\
\hline Neumann_delta_mod & 0.03149 & 0.00028 & 0.0015 \\
\hline alpha & 0.02989 & 0.32701 & 0.25276 \\
\hline C13_imag & 0.02871 & 0.25808 & $<0.00001$ \\
\hline T12_imag & 0.02769 & $<0.00001$ & 0.00008 \\
\hline s12_pha & 0.02711 & 1 & 1 \\
\hline s21_pha & 0.02683 & 1 & 1 \\
\hline s21 & 0.02674 & 0.77587 & 0.50734 \\
\hline delta2 & 0.02492 & 0.77587 & 0.64636 \\
\hline delta & 0.02390 & $<0.00001$ & $<0.00001$ \\
\hline asymetry & 0.02236 & 0.03288 & 0.03738 \\
\hline beta & 0.02071 & $<0.00001$ & $<0.00001$ \\
\hline entropy_shannon_P_norm & 0.02061 & $<0.00001$ & $<0.00001$ \\
\hline TSVM_alpha_s1 & 0.01742 & 1 & 1 \\
\hline TSVM_psi2 & 0.01626 & 0.16256 & 0.27638 \\
\hline Freeman_Dbl & 0.01603 & 0.31939 & 0.09656 \\
\hline anisotropy_lueneburg & 0.01599 & 0.00002 & $<0.00001$ \\
\hline s22_pha & 0.01318 & 1 & 1 \\
\hline TSVM_tau_m & 0.01307 & 1 & 0.37339 \\
\hline s11_pha & 0.01273 & 0.86671 & 1 \\
\hline alpha2 & 0.01132 & $<0.00001$ & 0.00014 \\
\hline s12 & 0.01085 & 1 & 1 \\
\hline TSVM_tau_m2 & 0.00957 & 0.46168 & 0.0836 \\
\hline reflectivity_11ov22 & 0.00941 & $<0.00001$ & $<0.00001$ \\
\hline Neumann_tau & 0.00916 & $<0.00001$ & $<0.00001$ \\
\hline TSVM_tau_m1 & 0.00877 & 1 & 1 \\
\hline
\end{tabular}




\begin{tabular}{|c|c|c|c|}
\hline SAR Variable & $\begin{array}{l}\text { Maximum Base- } \\
\text { Level Difference of } \\
\text { Means Score }\end{array}$ & $\begin{array}{c}\text { THSD p-value } \\
\text { (corrected with FDR) }\end{array}$ & $\begin{array}{c}\text { G-H p-value (corrected } \\
\text { with FDR) }\end{array}$ \\
\hline TSVM_tau_m3 & 0.00836 & 0.58757 & 0.18507 \\
\hline s11 & 0.00827 & 1 & 1 \\
\hline TSVM_phi_s & 0.00701 & 0.00003 & $<0.00001$ \\
\hline gamma3 & 0.00579 & 1 & 1 \\
\hline T13_real & 0.00522 & 1 & 0.95863 \\
\hline beta1 & 0.00512 & $<0.00001$ & $<0.00001$ \\
\hline TSVM_phi_s3 & 0.00505 & 0.0139 & 0.00033 \\
\hline TSVM_psi1 & 0.00470 & 0.00254 & 0.72142 \\
\hline anisotropy12 & 0.00468 & $<0.00001$ & $<0.00001$ \\
\hline gamma2 & 0.00451 & 1 & 0.83903 \\
\hline TSVM_alpha_s & 0.00423 & 1 & 1 \\
\hline p2 & 0.00363 & $<0.00001$ & $<0.00001$ \\
\hline T23_imag & 0.00341 & 1 & 1 \\
\hline T23_real & 0.00334 & 1 & 0.71098 \\
\hline gamma & 0.00333 & 1 & 1 \\
\hline combination_HA & 0.00316 & 0.00226 & 0.00011 \\
\hline gamma1 & 0.00294 & 1 & 1 \\
\hline C12_real & 0.00273 & 1 & 1 \\
\hline Neumann_psi & 0.00267 & 1 & 0.13376 \\
\hline alpha1 & 0.00223 & 1 & 1 \\
\hline TSVM_phi_s1 & 0.00193 & 0.0004 & $<0.00001$ \\
\hline C23_imag & 0.00156 & 1 & 1 \\
\hline TSVM_phi_s2 & 0.00137 & 1 & 1 \\
\hline T12_real & 0.00096 & $<0.00001$ & 0.00458 \\
\hline C23_real & 0.00091 & 1 & 0.58125 \\
\hline C12_imag & 0.00083 & 1 & 1 \\
\hline reflectivity_22ov11 & 0.00021 & 0.07127 & 0.00193 \\
\hline T13_imag & 0.00013 & 1 & 1 \\
\hline TSVM_psi3 & 0.00000 & 0.02808 & 0.03826 \\
\hline TSVM_psi & 0.00000 & 0.00052 & 0.6929 \\
\hline
\end{tabular}


Table 10.4: SAR variables maximum base-level difference of means scores for separating meteoric ice from other ocean covers.

\begin{tabular}{|c|c|c|c|}
\hline SAR Variable & $\begin{array}{c}\text { Maximum Base- } \\
\text { Level Difference of } \\
\text { Means Score } \\
\end{array}$ & $\begin{array}{c}\text { THSD p-value } \\
\text { (corrected with FDR) }\end{array}$ & $\begin{array}{c}\text { G-H p-value (corrected } \\
\text { with FDR) }\end{array}$ \\
\hline T22 & 0.25843 & $<0.00001$ & $<0.00001$ \\
\hline VanZyl3_Dbl & 0.25474 & $<0.00001$ & $<0.00001$ \\
\hline T22_mod & 0.25283 & $<0.00001$ & $<0.00001$ \\
\hline 12 & 0.24840 & $<0.00001$ & $<0.00001$ \\
\hline I21 & 0.24511 & $<0.00001$ & $<0.00001$ \\
\hline $\mathrm{I} 12$ & 0.24315 & $<0.00001$ & $<0.00001$ \\
\hline 13 & 0.23481 & $<0.00001$ & $<0.00001$ \\
\hline T33 & 0.23238 & $<0.00001$ & $<0.00001$ \\
\hline Freeman_Vol & 0.23231 & $<0.00001$ & $<0.00001$ \\
\hline T23_mod & 0.23230 & $<0.00001$ & $<0.00001$ \\
\hline VanZyl3_Vol & 0.23034 & $<0.00001$ & $<0.00001$ \\
\hline T33_mod & 0.22974 & $<0.00001$ & $<0.00001$ \\
\hline C22 & 0.22697 & $<0.00001$ & $<0.00001$ \\
\hline A12 & 0.19846 & $<0.00001$ & $<0.00001$ \\
\hline A21 & 0.19248 & $<0.00001$ & $<0.00001$ \\
\hline T13_mod & 0.18665 & $<0.00001$ & $<0.00001$ \\
\hline Krogager_Kd & 0.18481 & $<0.00001$ & $<0.00001$ \\
\hline I11 & 0.15288 & $<0.00001$ & $<0.00001$ \\
\hline s2_span & 0.15149 & $<0.00001$ & $<0.00001$ \\
\hline I22 & 0.13832 & $<0.00001$ & $<0.00001$ \\
\hline C11 & 0.13434 & $<0.00001$ & $<0.00001$ \\
\hline T12_imag & 0.13245 & $<0.00001$ & 0.00005 \\
\hline C13_imag & 0.12968 & $<0.00001$ & $<0.00001$ \\
\hline A11 & 0.12825 & $<0.00001$ & $<0.00001$ \\
\hline C33 & 0.12206 & $<0.00001$ & $<0.00001$ \\
\hline A22 & 0.12046 & $<0.00001$ & $<0.00001$ \\
\hline 11 & 0.11447 & $<0.00001$ & $<0.00001$ \\
\hline T11_mod & 0.11255 & $<0.00001$ & $<0.00001$ \\
\hline Krogager_Kh & 0.11224 & $<0.00001$ & $<0.00001$ \\
\hline $\mathrm{T} 11$ & 0.11077 & $<0.00001$ & $<0.00001$ \\
\hline $\mathrm{T} 12 \bmod$ & 0.11055 & $<0.00001$ & $<0.00001$ \\
\hline anisotropy_lueneburg & 0.10684 & $<0.00001$ & $<0.00001$ \\
\hline VanZyl3_Odd & 0.10451 & $<0.00001$ & $<0.00001$ \\
\hline lambda & 0.10352 & $<0.00001$ & $<0.00001$ \\
\hline pedestal & 0.09982 & $<0.00001$ & $<0.00001$ \\
\hline serd & 0.09960 & $<0.00001$ & $<0.00001$ \\
\hline serd_norm & 0.09921 & $<0.00001$ & $<0.00001$ \\
\hline anisotropy12 & 0.09863 & $<0.00001$ & $<0.00001$ \\
\hline Freeman_Dbl & 0.09805 & 0.0048 & 0.00082 \\
\hline TSVM_alpha_s & 0.09758 & 0.00992 & 0.05957 \\
\hline alpha & 0.09742 & $<0.00001$ & 0.00003 \\
\hline p1 & 0.09740 & $<0.00001$ & $<0.00001$ \\
\hline alpha2 & 0.09726 & $<0.00001$ & 0.00283 \\
\hline alpha1 & 0.09640 & 0.02543 & 0.0342 \\
\hline C13_real & 0.09554 & $<0.00001$ & $<0.00001$ \\
\hline
\end{tabular}




\begin{tabular}{|c|c|c|c|}
\hline SAR Variable & $\begin{array}{l}\text { Maximum Base- } \\
\text { Level Difference of } \\
\text { Means Score }\end{array}$ & $\begin{array}{c}\text { THSD p-value } \\
\text { (corrected with FDR) }\end{array}$ & $\begin{array}{c}\text { G-H p-value (corrected } \\
\text { with FDR) }\end{array}$ \\
\hline Freeman_Odd & 0.09485 & 0.00002 & $<0.00001$ \\
\hline alpha3 & 0.09319 & $<0.00001$ & $<0.00001$ \\
\hline entropy_shannon_norm & 0.09213 & $<0.00001$ & $<0.00001$ \\
\hline $\mathrm{p} 2$ & 0.08957 & $<0.00001$ & $<0.00001$ \\
\hline p3 & 0.08814 & $<0.00001$ & $<0.00001$ \\
\hline wishart_H_alpha_class_3 & 0.08786 & $<0.00001$ & $<0.00001$ \\
\hline polarisation_fraction & 0.08734 & $<0.00001$ & $<0.00001$ \\
\hline rvi & 0.08705 & $<0.00001$ & $<0.00001$ \\
\hline Krogager_Ks & 0.08612 & $<0.00001$ & $<0.00001$ \\
\hline C12_real & 0.08415 & 0.08885 & 0.00745 \\
\hline Neumann_delta_mod & 0.08300 & $<0.00001$ & $<0.00001$ \\
\hline entropy_shannon_P_norm & 0.08119 & $<0.00001$ & $<0.00001$ \\
\hline entropy & 0.07850 & $<0.00001$ & $<0.00001$ \\
\hline T13_real & 0.07575 & 0.05094 & 0.00922 \\
\hline entropy_shannon & 0.07494 & $<0.00001$ & $<0.00001$ \\
\hline TSVM_alpha_s1 & 0.07351 & 0.10776 & 0.13612 \\
\hline reflectivity_12ov22 & 0.07111 & $<0.00001$ & $<0.00001$ \\
\hline entropy_shannon_I_norm & 0.06709 & $<0.00001$ & $<0.00001$ \\
\hline combination_H1mA & 0.06292 & $<0.00001$ & $<0.00001$ \\
\hline TSVM_psi2 & 0.06154 & 0.11293 & 0.26783 \\
\hline reflectivity_12ov11 & 0.05897 & $<0.00001$ & $<0.00001$ \\
\hline entropy_shannon_I & 0.05874 & $<0.00001$ & 0.00005 \\
\hline combination_1mH1mA & 0.04780 & 0.00006 & 0.00027 \\
\hline entropy_shannon_P & 0.04563 & $<0.00001$ & $<0.00001$ \\
\hline TSVM_psi3 & 0.04017 & 0.01787 & 0.03535 \\
\hline T23_real & 0.03835 & 0.54787 & 0.21348 \\
\hline Neumann_psi & 0.03710 & 0.24858 & 0.03724 \\
\hline TSVM_psi1 & 0.03519 & 0.00002 & 0.47126 \\
\hline T23_imag & 0.03442 & 0.28186 & 0.0152 \\
\hline TSVM_tau_m1 & 0.03416 & 0.89109 & 0.46385 \\
\hline TSVM_alpha_s2 & 0.03247 & 0.00126 & 0.0081 \\
\hline delta3 & 0.03213 & 0.75664 & 0.76662 \\
\hline delta & 0.02934 & $<0.00001$ & 0.00004 \\
\hline T12_real & 0.02549 & $<0.00001$ & 0.0007 \\
\hline reflectivity_11ov22 & 0.02441 & $<0.00001$ & $<0.00001$ \\
\hline gamma & 0.02291 & 0.93217 & 0.98719 \\
\hline TSVM_tau_m3 & 0.02259 & 0.92004 & 0.33382 \\
\hline C23_real & 0.02238 & 0.58702 & 0.38017 \\
\hline gamma1 & 0.02201 & 0.99995 & 0.99997 \\
\hline TSVM_psi & 0.02149 & 0.00002 & 0.51813 \\
\hline asymetry & 0.02132 & 0.02106 & 0.03489 \\
\hline reflectivity_22ov12 & 0.02101 & 0.00137 & $<0.00001$ \\
\hline reflectivity_11ov12 & 0.01930 & 0.00299 & $<0.00001$ \\
\hline TSVM_alpha_s3 & 0.01794 & 0.00014 & 0.0006 \\
\hline TSVM_phi_s3 & 0.01696 & 0.00036 & 0.00121 \\
\hline reflectivity_22ov11 & 0.01686 & 0.27558 & 0.03523 \\
\hline beta & 0.01678 & $<0.00001$ & $<0.00001$ \\
\hline
\end{tabular}




\begin{tabular}{|c|c|c|c|}
\hline SAR Variable & $\begin{array}{l}\text { Maximum Base- } \\
\text { Level Difference of } \\
\text { Means Score }\end{array}$ & $\begin{array}{c}\text { THSD p-value } \\
\text { (corrected with FDR) }\end{array}$ & $\begin{array}{c}\text { G-H p-value (corrected } \\
\text { with FDR) }\end{array}$ \\
\hline s21_pha & 0.01588 & 0.92004 & 0.27976 \\
\hline combination_1mHA & 0.01494 & 0.00016 & $<0.00001$ \\
\hline delta2 & 0.01280 & 0.93927 & 0.98719 \\
\hline TSVM_phi_s & 0.00959 & 0.0001 & $<0.00001$ \\
\hline wishart_H_A_alpha_class_3 & 0.00861 & 0.67141 & 0.76799 \\
\hline Neumann_delta_pha & 0.00794 & 0.03161 & 0.00881 \\
\hline TSVM_tau_m & 0.00784 & 0.93217 & 0.98719 \\
\hline s22_pha & 0.00654 & 0.92004 & 0.98719 \\
\hline s12_pha & 0.00644 & 0.92004 & 0.45662 \\
\hline delta1 & 0.00589 & $<0.00001$ & $<0.00001$ \\
\hline Neumann_tau & 0.00561 & $<0.00001$ & $<0.00001$ \\
\hline beta1 & 0.00512 & $<0.00001$ & $<0.00001$ \\
\hline gamma2 & 0.00451 & 0.94416 & 0.78638 \\
\hline beta3 & 0.00391 & $<0.00001$ & $<0.00001$ \\
\hline gamma3 & 0.00370 & 0.92004 & 0.54546 \\
\hline s11 & 0.00354 & 0.99995 & 0.99997 \\
\hline combination_HA & 0.00316 & 0.00075 & 0.00013 \\
\hline TSVM_tau_m2 & 0.00311 & 0.37847 & 0.60313 \\
\hline s11_pha & 0.00303 & 0.80948 & 0.95273 \\
\hline beta2 & 0.00229 & $<0.00001$ & $<0.00001$ \\
\hline TSVM_phi_s1 & 0.00193 & 0.00018 & $<0.00001$ \\
\hline anisotropy & 0.00155 & 0.00422 & 0.00161 \\
\hline s22 & 0.00143 & 0.48364 & 0.76799 \\
\hline C12_imag & 0.00123 & 0.99995 & 0.99997 \\
\hline C23_imag & 0.00081 & 0.99995 & 0.99997 \\
\hline TSVM_phi_s2 & 0.00081 & 0.93217 & 0.87166 \\
\hline s12 & 0.00056 & 0.93927 & 0.9987 \\
\hline derd & 0.00054 & $<0.00001$ & $<0.00001$ \\
\hline s21 & 0.00052 & 0.29083 & 0.60161 \\
\hline derd_norm & 0.00033 & $<0.00001$ & $<0.00001$ \\
\hline T13_imag & 0.00012 & 0.99995 & 0.99997 \\
\hline
\end{tabular}


Table 10.5: SAR variables maximum base-level difference of means scores for separating MYI from other ocean covers.

\begin{tabular}{|c|c|c|c|}
\hline SAR Variable & $\begin{array}{c}\text { Maximum Base-Level } \\
\text { Difference of Means } \\
\text { Score }\end{array}$ & $\begin{array}{c}\text { THSD p-value } \\
\text { (corrected with FDR) }\end{array}$ & $\begin{array}{c}\text { G-H p-value (corrected } \\
\text { with FDR) }\end{array}$ \\
\hline Krogager_Kd & 0.12823 & $<0.00001$ & $<0.00001$ \\
\hline T23_mod & 0.12563 & 0.00002 & $<0.00001$ \\
\hline T33 & 0.12075 & 0.00011 & $<0.00001$ \\
\hline Freeman_Vol & 0.12066 & 0.00014 & $<0.00001$ \\
\hline T33_mod & 0.11926 & 0.0001 & $<0.00001$ \\
\hline C22 & 0.11758 & 0.0001 & $<0.00001$ \\
\hline A12 & 0.11690 & $<0.00001$ & $<0.00001$ \\
\hline 13 & 0.11528 & 0.00015 & $<0.00001$ \\
\hline A21 & 0.11295 & $<0.00001$ & $<0.00001$ \\
\hline VanZyl3_Vol & 0.11276 & $<0.00001$ & $<0.00001$ \\
\hline Krogager_Kh & 0.11224 & $<0.00001$ & $<0.00001$ \\
\hline 12 & 0.10656 & 0.00011 & $<0.00001$ \\
\hline $\mathrm{I} 12$ & 0.10071 & $<0.00001$ & $<0.00001$ \\
\hline I21 & 0.10048 & $<0.00001$ & $<0.00001$ \\
\hline serd & 0.09960 & 0.00388 & 0.00012 \\
\hline serd_norm & 0.09921 & 0.00387 & 0.00011 \\
\hline T22 & 0.09861 & $<0.00001$ & $<0.00001$ \\
\hline T22_mod & 0.09742 & $<0.00001$ & $<0.00001$ \\
\hline pedestal & 0.09441 & $<0.00001$ & $<0.00001$ \\
\hline entropy_shannon_norm & 0.09213 & $<0.00001$ & $<0.00001$ \\
\hline p3 & 0.08814 & $<0.00001$ & $<0.00001$ \\
\hline wishart_H_alpha_class_3 & 0.08786 & 0.0005 & $<0.00001$ \\
\hline polarisation_fraction & 0.08734 & 0.00806 & 0.0004 \\
\hline rvi & 0.08705 & $<0.00001$ & $<0.00001$ \\
\hline $\mathrm{p} 1$ & 0.08550 & 0.0667 & 0.01158 \\
\hline alpha & 0.08488 & 0.00302 & 0.00456 \\
\hline T13_mod & 0.08238 & 0.00032 & $<0.00001$ \\
\hline entropy_shannon_P_norm & 0.08119 & $<0.00001$ & $<0.00001$ \\
\hline anisotropy_lueneburg & 0.08079 & $<0.00001$ & $<0.00001$ \\
\hline Krogager_Ks & 0.07974 & 0.00003 & 0.00025 \\
\hline entropy & 0.07850 & $<0.00001$ & $<0.00001$ \\
\hline TSVM_phi_s3 & 0.07743 & 0.00052 & 0.00131 \\
\hline anisotropy12 & 0.07611 & 0.16104 & 0.044 \\
\hline entropy_shannon & 0.07494 & $<0.00001$ & $<0.00001$ \\
\hline C33 & 0.07481 & 0.0021 & 0.00013 \\
\hline Neumann_delta_mod & 0.07135 & $<0.00001$ & 0.00004 \\
\hline reflectivity_12ov22 & 0.07111 & $<0.00001$ & $<0.00001$ \\
\hline alpha3 & 0.07080 & 0.08862 & 0.00412 \\
\hline p2 & 0.07078 & $<0.00001$ & $<0.00001$ \\
\hline C11 & 0.06974 & 0.0019 & $<0.00001$ \\
\hline s2_span & 0.06764 & 0.00388 & 0.00008 \\
\hline TSVM_alpha_s & 0.06733 & 0.03936 & 0.03129 \\
\hline entropy_shannon_I_norm & 0.06709 & $<0.00001$ & 0.00013 \\
\hline 11 & 0.06692 & 0.00458 & 0.00028 \\
\hline $\mathrm{T} 11$ & 0.06544 & 0.00458 & 0.00024 \\
\hline
\end{tabular}




\begin{tabular}{|c|c|c|c|}
\hline SAR Variable & $\begin{array}{c}\text { Maximum Base-Level } \\
\text { Difference of Means } \\
\text { Score }\end{array}$ & $\begin{array}{c}\text { THSD p-value } \\
\text { (corrected with FDR) }\end{array}$ & $\begin{array}{c}\text { G-H p-value (corrected } \\
\text { with FDR) }\end{array}$ \\
\hline T11_mod & 0.06534 & 0.00458 & 0.00024 \\
\hline combination_H1mA & 0.06292 & $<0.00001$ & $<0.00001$ \\
\hline C13_real & 0.05957 & 0.00902 & 0.00039 \\
\hline reflectivity_12ov11 & 0.05897 & $<0.00001$ & 0.00009 \\
\hline entropy_shannon_I & 0.05874 & $<0.00001$ & 0.0004 \\
\hline lambda & 0.05789 & 0.00806 & 0.00034 \\
\hline VanZyl3_Odd & 0.05700 & 0.00797 & 0.00035 \\
\hline A22 & 0.05672 & 0.00005 & 0.00015 \\
\hline delta1 & 0.05237 & $<0.00001$ & $<0.00001$ \\
\hline A11 & 0.05169 & 0.00007 & 0.00002 \\
\hline T12_mod & 0.05074 & $<0.00001$ & $<0.00001$ \\
\hline VanZyl3_Dbl & 0.05031 & $<0.00001$ & $<0.00001$ \\
\hline alpha1 & 0.05031 & 0.02479 & 0.02531 \\
\hline $\mathrm{I} 11$ & 0.05023 & 0.00503 & $<0.00001$ \\
\hline I22 & 0.04959 & 0.00755 & 0.0001 \\
\hline delta3 & 0.04800 & 0.23324 & 0.09234 \\
\hline entropy_shannon_P & 0.04563 & $<0.00001$ & 0.00003 \\
\hline Neumann_delta_pha & 0.04528 & 0.03551 & 0.00924 \\
\hline Freeman_Odd & 0.04279 & 0.02075 & 0.00057 \\
\hline combination_1mH1mA & 0.03463 & 0.23208 & 0.12593 \\
\hline TSVM_alpha_s2 & 0.03247 & 0.08175 & 0.01069 \\
\hline TSVM_alpha_s1 & 0.03143 & 0.06276 & 0.03663 \\
\hline combination_HA & 0.03072 & 0.01624 & 0.00079 \\
\hline C13_imag & 0.02871 & 0.00008 & 0.00102 \\
\hline T12_imag & 0.02769 & 0.9724 & 0.31232 \\
\hline delta2 & 0.02470 & 0.71725 & 0.60368 \\
\hline delta & 0.02390 & $<0.00001$ & $<0.00001$ \\
\hline asymetry & 0.02236 & 0.9724 & 0.99435 \\
\hline beta1 & 0.02210 & $<0.00001$ & $<0.00001$ \\
\hline wishart_H_A_alpha_class_3 & 0.02139 & 0.23196 & 0.21119 \\
\hline TSVM_phi_s1 & 0.02137 & 0.00009 & $<0.00001$ \\
\hline reflectivity_22ov12 & 0.02101 & 0.1135 & 0.00064 \\
\hline reflectivity_11ov12 & 0.01930 & 0.10993 & 0.00037 \\
\hline alpha2 & 0.01813 & $<0.00001$ & 0.00026 \\
\hline TSVM_alpha_s3 & 0.01794 & 0.00006 & $<0.00001$ \\
\hline beta & 0.01678 & $<0.00001$ & $<0.00001$ \\
\hline TSVM_psi2 & 0.01626 & 0.99999 & 0.93522 \\
\hline s21_pha & 0.01588 & 0.99999 & 0.51123 \\
\hline combination_1mHA & 0.01494 & 0.00576 & $<0.00001$ \\
\hline Neumann_psi & 0.01169 & 0.12512 & 0.20968 \\
\hline Freeman_Dbl & 0.01067 & 0.43844 & 0.19007 \\
\hline TSVM_tau_m & 0.00961 & 0.99999 & 0.38486 \\
\hline reflectivity_11ov22 & 0.00941 & $<0.00001$ & $<0.00001$ \\
\hline Neumann_tau & 0.00916 & $<0.00001$ & $<0.00001$ \\
\hline TSVM_tau_m1 & 0.00867 & 0.67364 & 0.60368 \\
\hline TSVM_tau_m3 & 0.00836 & 0.6756 & 0.00175 \\
\hline s12 & 0.00770 & 0.99999 & 0.99997 \\
\hline
\end{tabular}




\begin{tabular}{rccc}
\hline SAR Variable & $\begin{array}{c}\text { Maximum Base-Level } \\
\text { Difference of Means } \\
\text { Score }\end{array}$ & $\begin{array}{c}\text { THSD p-value } \\
\text { (corrected with FDR) }\end{array}$ & $\begin{array}{c}\text { G-H p-value (corrected } \\
\text { with FDR) }\end{array}$ \\
\hline TSVM_psi & 0.00714 & 0.00102 & 0.69813 \\
TSVM_phi_s & 0.00701 & 0.00002 & $<0.00001$ \\
s22 & 0.00678 & 0.15534 & 0.3031 \\
s12_pha & 0.00644 & 0.99999 & 0.48797 \\
gamma2 & 0.00631 & 0.75944 & 0.00578 \\
gamma3 & 0.00579 & 0.9724 & 0.85031 \\
TSVM_psi3 & 0.00573 & 0.9724 & 0.99997 \\
gamma1 & 0.00527 & 0.99999 & 0.99997 \\
s11_pha & 0.00440 & 0.99999 & 0.99997 \\
beta3 & 0.00391 & $<0.00001$ & $<0.00001$ \\
s22_pha & 0.00383 & 0.99999 & 0.99997 \\
s11 & 0.00351 & 0.99999 & 0.99997 \\
T23_imag & 0.00341 & 0.15534 & 0.10332 \\
T23_real & 0.00334 & 0.17139 & 0.31666 \\
TSVM_tau_m2 & 0.00311 & 0.53132 & 0.43058 \\
gamma & 0.00310 & 0.99999 & 0.99997 \\
TSVM_psi1 & 0.00267 & 0.00435 & 0.73154 \\
beta2 & 0.00229 & $<0.00001$ & $<0.00001$ \\
C12_real & 0.00173 & 0.00197 & 0.01105 \\
C23_real & 0.00166 & 0.67364 & 0.76642 \\
anisotropy & 0.00155 & 0.0106 & 0.00003 \\
T13_real & 0.00151 & 0.00374 & 0.01261 \\
TSVM_phi_s2 & 0.00137 & 0.9724 & 0.87952 \\
T12_real & 0.00096 & $<0.00001$ & 0.00382 \\
C12_imag & 0.00083 & 0.99999 & 0.99997 \\
C23_imag & 0.00081 & 0.99999 & 0.97503 \\
derd & 0.00054 & $<0.00001$ & $<0.00001$ \\
s21 & 0.00052 & 0.36868 & 0.55606 \\
derd_norm & 0.00033 & $<0.00001$ & 0.00001 \\
T13_imag & 0.00021 & 0.05001 & 0.00159 \\
reflectivity22ov11 & 0.00013 & & \\
\hline
\end{tabular}




\section{Appendix E}

\subsection{Accuracy Assessments of the Nine Testing SAR Images}

Table 11.1: Accuracy assessment of RS2 FQ14 acquired 2010/09/11-23:50:36. Kappa was 0.17.

\begin{tabular}{|c|c|c|c|c|c|c|c|}
\hline & \multicolumn{4}{|c|}{ Reference Data } & \multirow{2}{*}{ Total Segments } & \multirow{2}{*}{ User Accuracy (\%) } \\
\hline & & water & FYI & meteoric & MYI & & \\
\hline \multirow{4}{*}{ 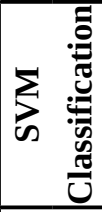 } & water & 0 & 0 & 0 & 0 & 0 & 0 \\
\hline & FYI & 4 & 1 & 0 & 0 & 5 & 20.0 \\
\hline & meteoric & 0 & 0 & 0 & 4 & 4 & 0 \\
\hline & MYI & 2 & 0 & 3 & 19 & 24 & 79.1 \\
\hline \multicolumn{2}{|c|}{ Total Segments } & 6 & 1 & 3 & 23 & 33 & \\
\hline \multicolumn{2}{|c|}{ Producer Accuracy (\%) } & 0 & 100.0 & 0 & 82.6 & & \\
\hline
\end{tabular}

Table 11.2: Accuracy assessment of RS2 FQ6W acquired 2012/06/14-13:28:38. Kappa was -0.07.

\begin{tabular}{|c|c|c|c|c|c|c|c|}
\hline & \multicolumn{4}{|c|}{ Reference Data } & \multirow{2}{*}{ Total Segments } & \multirow{2}{*}{ User Accuracy (\%) } \\
\hline & & water & FYI & meteoric & MYI & & \\
\hline \multirow{4}{*}{ 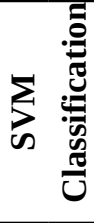 } & water & 0 & 0 & 0 & 0 & 0 & 0 \\
\hline & FYI & 0 & 20 & 3 & 0 & 23 & 86.9 \\
\hline & meteoric & 0 & 0 & 0 & 0 & 0 & 0 \\
\hline & MYI & 0 & 10 & 0 & 0 & 10 & 0 \\
\hline \multicolumn{2}{|c|}{ Total Segments } & 0 & 30 & 3 & 0 & 33 & \\
\hline \multicolumn{2}{|c|}{ Producer Accuracy (\%) } & 0 & 66.6 & 0 & 0 & & \\
\hline
\end{tabular}

Table 11.3: Accuracy assessment of RS2 FQ17W acquired 2012/10/29-21:46:43. Kappa was 0.42.

\begin{tabular}{|c|c|c|c|c|c|c|c|}
\hline & \multicolumn{4}{|c|}{ Reference Data } & \multirow{2}{*}{ Total Segments } & \multirow{2}{*}{ User Accuracy (\%) } \\
\hline & & water & FYI & meteoric & MYI & & \\
\hline \multirow{4}{*}{ 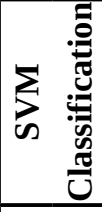 } & water & 29 & 0 & 0 & 0 & 29 & 100 \\
\hline & FYI & 0 & 0 & 3 & 0 & 3 & 0 \\
\hline & meteoric & 0 & 0 & 0 & 0 & 0 & 0 \\
\hline & MYI & 0 & 0 & 7 & 0 & 7 & 0 \\
\hline \multicolumn{2}{|c|}{ Total Segments } & 29 & 0 & 10 & 0 & 39 & \\
\hline \multicolumn{2}{|c|}{ Producer Accuracy (\%) } & 100.0 & 0 & 0 & 0 & & \\
\hline
\end{tabular}


Table 11.4: Accuracy assessment of RS2 FQ19W acquired 2013/04/08-21:50:38. Kappa was 0.35.

\begin{tabular}{|c|c|c|c|c|c|c|c|}
\hline & \multicolumn{4}{|c|}{ Reference Data } & \multirow{2}{*}{ Total Segments } & \multirow{2}{*}{ User Accuracy (\%) } \\
\hline & & water & FYI & meteoric & MYI & & \\
\hline \multirow{4}{*}{ 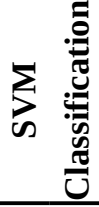 } & water & 0 & 0 & 0 & 0 & 0 & 0 \\
\hline & FYI & 0 & 29 & 1 & 0 & 30 & 96.6 \\
\hline & meteoric & 0 & 0 & 0 & 0 & 0 & 0 \\
\hline & MYI & 0 & 1 & 4 & 0 & 5 & 0 \\
\hline \multicolumn{2}{|c|}{ Total Segments } & 0 & 30 & 5 & 0 & 35 & \\
\hline \multicolumn{2}{|c|}{ Producer Accuracy (\%) } & 0 & 96.6 & 0 & 0 & & \\
\hline
\end{tabular}

Table 11.5: Accuracy assessment of RS2 FQ4W acquired 2013/06/17-11:16:08. Kappa was -0.12.

\begin{tabular}{|c|c|c|c|c|c|c|c|}
\hline & \multicolumn{4}{|c|}{ Reference Data } & \multirow{2}{*}{ Total Segments } & \multirow{2}{*}{ User Accuracy (\%) } \\
\hline & & water & FYI & meteoric & MYI & & \\
\hline \multirow{4}{*}{ 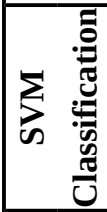 } & water & 0 & 0 & 0 & 0 & 0 & 0 \\
\hline & FYI & 1 & 2 & 3 & 0 & 6 & 33.3 \\
\hline & meteoric & 0 & 0 & 0 & 0 & 0 & 0 \\
\hline & MYI & 0 & 19 & 0 & 1 & 20 & 5.0 \\
\hline \multicolumn{2}{|c|}{ Total Segments } & 1 & 21 & 3 & 1 & 26 & \\
\hline \multicolumn{2}{|c|}{ Producer Accuracy (\%) } & 0 & 9.5 & 0 & 100 & & \\
\hline
\end{tabular}

Table 11.6: Accuracy assessment of RS2 FQ24 acquired 2013/06/29-21:58:59. Kappa was 0.0.

\begin{tabular}{|c|c|c|c|c|c|c|c|}
\hline & \multicolumn{4}{|c|}{ Reference Data } & \multirow{2}{*}{ Total Segments } & \multirow{2}{*}{ User Accuracy (\%) } \\
\hline & & water & FYI & meteoric & MYI & & \\
\hline \multirow{4}{*}{ 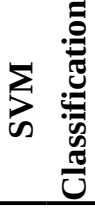 } & water & 0 & 0 & 0 & 0 & 0 & 0 \\
\hline & FYI & 0 & 30 & 13 & 0 & 43 & 69.7 \\
\hline & meteoric & 0 & 0 & 0 & 0 & 0 & 0 \\
\hline & MYI & 0 & 0 & 0 & 0 & 0 & 0 \\
\hline \multicolumn{2}{|c|}{ Total Segments } & 0 & 30 & 13 & 0 & 43 & \\
\hline \multicolumn{2}{|c|}{ Producer Accuracy (\%) } & 0 & 100 & 0 & 0 & & \\
\hline
\end{tabular}

Table 11.7: Accuracy assessment of RS2 FQ22 acquired 2013/07/02-22:12:26. Kappa was 0.27.

\begin{tabular}{|c|c|c|c|c|c|c|c|}
\hline & \multicolumn{4}{|c|}{ Reference Data } & \multirow[b]{2}{*}{ Total Segments } & \multirow{2}{*}{ User Accuracy (\%) } \\
\hline & & water & FYI & meteoric & MYI & & \\
\hline \multirow{4}{*}{ 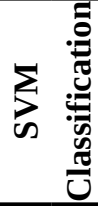 } & water & 2 & 1 & 0 & 0 & 3 & 66.6 \\
\hline & FYI & 0 & 22 & 6 & 1 & 29 & 75.8 \\
\hline & meteoric & 0 & 0 & 0 & 0 & 0 & 0 \\
\hline & MYI & 0 & 0 & 0 & 0 & 0 & 0 \\
\hline \multicolumn{2}{|c|}{ Total Segments } & 2 & 23 & 6 & 1 & 32 & \\
\hline \multicolumn{2}{|c|}{ Producer Accuracy (\%) } & 100 & 95.6 & 0 & 0 & & \\
\hline
\end{tabular}


Table 11.8: Accuracy assessment of RS2 FQ15W acquired 2012/06/14-21:42:37. Kappa was 0.21.

\begin{tabular}{|c|c|c|c|c|c|c|c|}
\hline & \multicolumn{4}{|c|}{ Reference Data } & \multirow{2}{*}{ Total Segments } & \multirow{2}{*}{ User Accuracy (\%) } \\
\hline & & water & FYI & meteoric & MYI & & \\
\hline \multirow{4}{*}{ 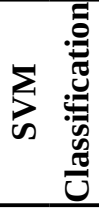 } & water & 4 & 0 & 0 & 0 & 4 & 100 \\
\hline & FYI & 1 & 24 & 17 & 0 & 42 & 57.1 \\
\hline & meteoric & 0 & 0 & 0 & 0 & 0 & 0 \\
\hline & MYI & 0 & 1 & 0 & 0 & 1 & 0 \\
\hline \multicolumn{2}{|c|}{ Total Segments } & 5 & 25 & 17 & 0 & 47 & \\
\hline \multicolumn{2}{|c|}{ Producer Accuracy (\%) } & 80.0 & 96.0 & 0 & 0 & & \\
\hline
\end{tabular}

Table 11.9: Accuracy assessment of RS2 FQ13W acquired 2013/03/26-11:36:17. Kappa was 0.01.

\begin{tabular}{|c|c|c|c|c|c|c|c|}
\hline & \multicolumn{4}{|c|}{ Reference Data } & \multirow{2}{*}{ Total Segments } & \multirow{2}{*}{ User Accuracy (\%) } \\
\hline & & water & FYI & meteoric & MYI & & \\
\hline \multirow{4}{*}{ 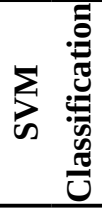 } & water & 0 & 0 & 0 & 0 & 0 & 0 \\
\hline & FYI & 0 & 10 & 2 & 0 & 12 & 83.3 \\
\hline & meteoric & 0 & 0 & 0 & 0 & 0 & 0 \\
\hline & MYI & 0 & 4 & 1 & $\underline{0}$ & 5 & 0 \\
\hline \multicolumn{2}{|c|}{ Total Segments } & 0 & 14 & 3 & 0 & 17 & \\
\hline \multicolumn{2}{|c|}{ Producer Accuracy (\%) } & 0 & 71.4 & 0 & 0 & & \\
\hline
\end{tabular}

